\title{
Quantum Supergroups and Canonical Bases
}

\author{
Sean Ian Clark \\ Oak Hill, Virginia
}

B.S., College of William and Mary, 2009

A Dissertation presented to the Graduate Faculty of the University of Virginia in Candidacy for the Degree of Doctor of Philosophy

Department of Mathematics

University of Virginia

May, 2014 


\begin{abstract}
Drinfeld and Jimbo introduced quantum groups as deformations of the universal enveloping algebras associated to semi-simple and Kac-Moody Lie algebras. The study of the structure of these algebras lead to the discovery of canonical bases by Lusztig. These bases have many desirable properties, and recently have played an important role in the development of categorified quantum groups.

This dissertation studies the structures and canonical bases for quantum supergroups of anisotropic type; that is, quantum groups associated to Kac-Moody Lie superalgebras with no isotropic odd roots. We proceed by utilizing the framework of covering quantum groups, which are algebras with two parameters, namely the quantum parameter $q$ and half parameter $\pi$ satisfying $\pi^{2}=1$, which interpolates between a Drinfeld-Jimbo quantum group (the $\pi=1$ case) and a quantum supergroup (the $\pi=-1$ case). A version of these algebras was first introduced by Hill-Wang in the context of categorifications of quantum groups.

We develop analogues of several classical quantum group constructions, including a (braided) Hopf algebra structure and a quasi- $\mathcal{R}$-matrix which intertwines two coproducts. We also define an analogue of the BGG category $\mathcal{O}$ and show that the integrable modules in $\mathcal{O}$ are completely reducible. The covering quantum group admits a bar-involution $\bar{q}=\pi q^{-1}$ and a bar-invariant integral form. We construct canonical bases for integrable modules of $\mathcal{O}$ and for the half-quantum covering group, generalizing Kashiwara's grand loop and globalization constructions. This canonical basis is then extended to a modified form of the quantum covering group à la Lusztig. Specializing our constructions to $\pi=-1$ yields the first examples of canonical bases known for quantum supergroups.
\end{abstract}




\section{Acknowledgements}

I would like to express my immense gratitude to my advisor Weiqiang Wang. The impact of his advice and guidance on both my appreciation for mathematics and my skills as a researcher cannot be overstated. From his example, I am inspired to be a better researcher and collaborator, and I would be thrilled to one day be half as good of an advisor to my students as he has been to me.

I would also like to thank David Hill. I am grateful for his generosity of his time and ideas, both for our collaborations and for my professional development. I also thank Aaron Lauda, Yiqiang Li, and Zhaobing Fan for the collaborations and conversations that led to several of the ideas in this document.

I am thankful for the camaraderie and friendship of my peers at UVA, and specifically my academic siblings Yung-Ning Peng, Constance Baltera, Huanchen Bao, and Chun-Ju Lai. I also thank my friends, and in particular: Andrew Hale and Jack Rollins, for their abiding friendship and encouragement; Josh Schwartz for making the past five years immeasurably more fun; and Melissa McReynolds for her constant love and support, and especially for her patience.

Finally, I owe everything to my family. Thank you for filling my life with joy, for supporting my mathematical aspirations, and for the little blue calculator that somehow led to this dissertation. I dedicate this work to my earliest math tutor, my constant co-conspirator, and my best friend: my brother, Andrew. 


\section{Contents}

1 Introduction 1

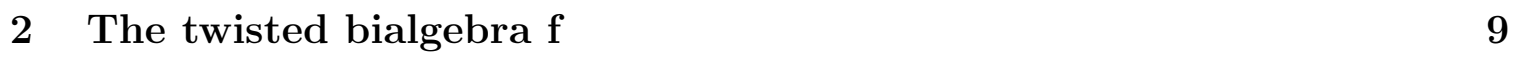

2.1 The Cartan datum . . . . . . . . . . . . . . . . . 9

2.2 Quantum parameters ................... 11

2.3 The algebras ' $\mathbf{f}$ and $\mathbf{f} \ldots \ldots \ldots \ldots$. . . . . . . . . . . . . . .

2.4 Differentials and Serre relations . . . . . . . . . . . 20

2.5 The twistor isomorphism . . . . . . . . . . . . 25

3 The quantum covering group $U$

3.1 The algebras ${ }^{\prime} \mathbf{U}$ and $\mathbf{U} \ldots \ldots . \ldots . \ldots . \ldots . \ldots 31$

3.2 Hopf Structure . . . . . . . . . . . . . . . . 36

3.3 Triangular Decomposition . . . . . . . . . . . . . . . . 39

3.4 The twistor automorphism of $\mathbf{U} \ldots \ldots . \ldots . \ldots 43$

3.5 The categories $\mathcal{C}$ and $\mathcal{O} \ldots \ldots \ldots$. . . . . . . . . . 44

3.6 The twistor and modules . . . . . . . . . . . . . . . . 49

3.7 The quasi- $\mathcal{R}$-matrix . . . . . . . . . . . . . . . 54

3.8 The quantum Casimir . . . . . . . . . . . . . . . 59

3.9 Complete reducibility in $\mathcal{O}_{\text {int }} \ldots \ldots \ldots$. . . . . . . . . 61

4 Crystal basis and the grand loop $\quad 64$

4.1 The $(q, \pi)$-boson superalgebra . . . . . . . . . . . 64 
4.2 Crystal bases . . . . . . . . . . . . . . . . . . . . 69

4.3 Tensor Product Rule . . . . . . . . . . . . . . . . . . . . . . . 72

4.4 Polarization ......................... 75

4.5 Main statements and grand loop argument . . . . . . . . . . . . . . . 77

4.6 Further properties of the polarization . . . . . . . . . . . 79

4.7 Twistors and the crystal structure . . . . . . . . . . . . . 83

4.8 Canonical bases . . . . . . . . . . . . . . . . 86

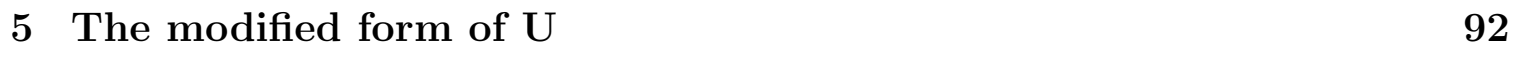

5.1 The modified form . . . . . . . . . . . . . . . . . . . . 92

5.2 A bar involution on $N\left(\lambda, \lambda^{\prime}\right) \ldots \ldots \ldots$

5.3 The canonical basis of $N\left(\lambda, \lambda^{\prime}\right) \quad \ldots \ldots \ldots$

5.4 Cancellation and stability . . . . . . . . . . . . . . 104

5.5 The canonical basis of $\dot{\mathbf{U}} \ldots \ldots$. . . . . . . . . . . . . . 108

5.6 The bilinear form on $\dot{\mathbf{U}} \ldots \ldots \ldots$. . . . . . . . . . . . . . 112

$\begin{array}{ll}\text { A Twisted Bialgebras } & 120\end{array}$

A.1 Definitions . . . . . . . . . . . . . . . . . 120

A.2 The Fan-Li transformation . . . . . . . . . . . . . . . . . 127 


\section{Chapter 1}

\section{Introduction}

1.1 The quantum groups associated to a Kac-Moody Lie algebra $\mathfrak{g}$ have been studied intensely since they were introduced in the seminal work of Drinfeld [D] and Jimbo [Ji]. These Hopf algebras have interesting and fruitful connections to a wide array of topics; indeed, while the notion of a quantum groups originally motivated by mathematical physics, they also are intimately related to Lie theory, modular representation theory [Lu2], knot invariants [RT], categorification, and so on.

The connection to categorification germinated from the discovery of a remarkable basis, called the canonical basis, for the negative half of the quantum group associated to $\mathfrak{g}$. This basis was discovered by Lusztig [Lu1], using geometric methods of perverse sheaves on quiver representations, and subsequently by Kashiwara [Kas1], using algebraic constructions called crystal bases. One of its remarkable properties is that if the associated Lie algebra $\mathfrak{g}$ has a symmetric generalized Cartan matrix, the structure coefficients with respect to the canonical basis lie in $\mathbb{N}\left[q, q^{-1}\right]$, where $q$ is the quantum parameter. This fact, which is a consequence of the geometry utilized by Lusztig, directly suggests a categorification and such a categorification has been produced in the work of Khovanov-Lauda [KL] and Rouquier [R] using quiver Hecke algebras. Since then, there has been a flurry of developments in categorification; see for example [We, BK, VV]. 
1.2 Given the physical origins of the study of quantum groups, a natural extension of the study of quantum groups associated to Kac-Moody Lie algebras would be to study quantum supergroups associated to Lie superalgebras (see [Kac, ChW] for more on Lie superalgebras). In fact, many papers have been produced defining quantum supergroups and determining many of their properties [BKM, Ge, Y1, Y2, Z]. Unfortunately, the more complicated structure of Lie superalgebras is reflected in the structure of their associated quantum groups. Despite the construction of crystal bases [BKK, Kw, MZ], no examples of canonical bases have been known for quantum supergroups, and indeed the prevailing expectation was that they did not exist.

On the other hand, some recent developments in categorification suggest the existence of canonical bases. In a paper of Kang-Kashiwara-Tsuchioka [KKT], it was suggested that quiver Hecke superalgebras, a natural super-analogue of KLR algebra which have also been studied in [Wa, EKL], could also be used to categorify KacMoody quantum groups (this conjecture was verified by Kang-Kashiwara-Oh [KKO]). However, any superalgebra comes equipped with a $\mathbb{Z} / 2 \mathbb{Z}$ grading, called the parity grading, and this grading induces functors on the representation categories. The categorification proposed in $[\mathrm{KKT}]$ essentially forgets this additional structure to obtain a non-super decategorification.

A key insight of Hill-Wang [HW] was that this parity could be used to encode a new additional "half-parameter" $\pi$ (satisfying $\pi^{2}=1$ ). If we consider quiver Hecke superalgebras associated to Cartan datums of anisotropic type - that is, a Cartan datum which is $\mathbb{Z} / 2 \mathbb{Z}$-graded and has with no isotropic odd roots - and we keep track of the parity functor, we can obtain a categorification of a half-quantum covering group. This half-quantum covering group is an algebra with two parameters, namely the (generic) quantum parameter $q$ and half parameter $\pi$ satisfying $\pi^{2}=1$, which specializes to a half-quantum group at $\pi=1$, and a half-quantum supergroup at $\pi=-1$. This perspective implies that there is at least a notion of a categorical canonical basis for the half-quantum supergroup arising from the indecomposable 
projective modules of the associated quiver Hecke superalgebra.

1.3 Motivated by this result, we examined the quantum group associated to $\mathfrak{o s p}(1 \mid 2)[\mathrm{ClW}]$. As this is the simplest anisotropic type quantum group and had already been studied in the literature, any hope for a canonical basis would start there. However, our investigation lead us to two variant definitions of the quantum group which each captured different sets of finite-dimensional simple modules. Combining them led to the construction of canonical bases for all the simple modules, as well as a canonical basis on a modified form of the quantum supergroup.

An important aspect of these results is that all of our constructions could be defined using the half-parameter $\pi$ such that structure coefficients, when suitably interpreted, are positive. It was conjectured [ClW] that, in the rank 1 case, a notion of a categorified modified quantum covering group could be constructed analogously to [Lau], and indeed this has been accomplished by Ellis-Lauda [EL]. Moreover, it is hoped that such categorifications could provide a categorical explanation for the existence of odd Khovanov homology [ORS], an idea which is corroborated by some upcoming work of Mikhaylov-Witten [MW].

1.4 It is the combination of the perspectives in [ClW, HW] which leads us to the definition of the quantum covering group, the main object of study in this dissertation. All of the main results stated in this dissertation were originally published in the sequence of papers [CHW1, CHW2, C] (also cf. [CFLW, CHW3]). Here we will present a coherent narrative of these results.

The quantum covering group $\mathbf{U}$ associated to an anisotropic Cartan datum is an algebra over the ring $\mathbb{Q}(q)^{\pi}=\mathbb{Q}(q) \oplus \mathbb{Q}(q) \pi$, where $q$ is the quantum parameter (in the usual sense) and $\pi$ is again a "half-parameter" satisfying $\pi^{2}=1$. Just as with half-quantum covering groups of $[\mathrm{HW}]$, the main idea is that the quantum covering group interpolates between usual quantum group at $\pi=1$ and a quantum supergroup 
at $\pi=-1$.

Our definition of a quantum supergroup is new, but is closely related to the definitions which have appeared in the literature. The main difference from these standard definitions is the addition of new generators to the Cartan part. To wit, a DrinfeldJimbo quantum group associated to $\mathfrak{g}$ has a subalgebra generated by elements of the form $q^{h}$, where $h$ is an element of the Cartan subalgebra $\mathfrak{h} \subset \mathfrak{g}$ and $q^{h}$ acts on a $\lambda$ weight space by $q^{\lambda(h)}$. Our quantum covering groups additionally have elements of the form $\pi^{h}$. Some important benefits of our definition is that the quantum supergroups are defined over $\mathbb{Q}(q)$, afford a $\mathbb{Z}\left[q, q^{-1}\right]$-integral form, and admit simple integrable modules for all dominant weights (compare with [Z, Je]).

The interpolation perspective of quantum covering groups allows us to develop the structure theory the quantum supergroups in tandem to that of quantum groups, and in particular most of the structural features (e.g. the triangular decomposition, the quantum Casimir operator and the quasi- $\mathcal{R}$-matrix) can be developed in the covering algebra setting. Moreover, we define a notion of a category $\mathcal{O}$ for $\mathbf{U}$, which is a direct sum of categories $\mathcal{O}_{ \pm}$of representations of the $\pi= \pm 1$ specializations of $\mathbf{U}$. On the other hand, a natural subclass of $\mathbf{U}$-modules are the $\pi$-free modules; that is, modules which are free with respect to the coefficient $\operatorname{ring} \mathbb{Q}(q)^{\pi}$. This leads us to our first main result.

Theorem A. Let $\mathbf{U}$ be the quantum covering group associated to an anisotropic Cartan datum. For each dominant weight $\lambda$, there is a unique simple $\pi$-free integrable module $V(\lambda) \in \mathcal{O}$ of highest weight $\lambda$. Moreover, any integrable $\pi$-free module $M \in \mathcal{O}$ is a direct sum of such simple modules.

1.5 A strong understanding of the structure of the quantum covering group $\mathbf{U}$ begins with first understanding the structure of $\mathbf{U}^{-}$, the half-quantum group associated to the negative roots. A useful abstraction of this algebra (which is isomorphic the half-quantum group) is the twisted bi-algebra $\mathbf{f}$, which was introduced for quantum 
groups by Lusztig. These algebras enjoy several useful structures, such as a (twisted) co-product, a non-degenerate bilinear form which entwines the multiplication and coproduct, and auxiliary structures such quantum derivations. To find a canonical basis, we would also need an integral form of $\mathbf{f}$, and an essential fact (which was first observed in [HW]) is that if we use the unorthodox bar involution $q \mapsto \pi q^{-1}$, this algebra has a natural bar-invariant integral form.

One main intent in our study of these algebras is to show that a reasonable intrinsic definition of a canonical basis exists, and to determine its properties. In particular, this demonstrates (for the first time) a family of quantum supergroups admitting canonical bases.

Theorem B. Let $\mathbf{U}$ be the quantum covering group associated to an anisotropic Cartan datum. Then the half-quantum group $\mathbf{U}^{-}$admits a canonical basis $\mathbb{B}$. Moreover, for each dominant weight $\lambda$ and associated simple module $V(\lambda)$ with highest weight vector $\eta$, the set $\{b \eta: b \in \mathbb{B}\} \backslash 0$ is a basis of $V(\lambda)$.

To prove this result, we have two potential paths to follow. The first would be to attempt to mimic Lusztig's geometric approach; however, there does not seem to be any geometry to use in the super case. Given the previous successes of crystal basis theory in the super world, we instead use Kashiwara's grand loop argument to construct crystal bases. Many essential arguments for this proof almost carry over exactly from [Kas1] with minor bookkeeping, but there are some significant deviations, most notably to obtain the globalization part of the argument.

Indeed, in the covering setting it is much harder to show that the crystal lattices are invariant under a certain antiautomorphism of $\mathbf{f}$, a property which features crucially in constructing the canonical bases. To obtain this result, we use techniques from [CFLW], which were motivated by the two-parameter construction given by FanLi [FL]. In particular, we construct some remarkable isomorphisms, called twistors, over a complexification of our algebra which allow us to directly link the super and non-super versions of our quantum group. These isomorphisms are used in an essen- 
tial way to prove the existence of the canonical basis; moreover, we show that they preserve the canonical basis in a suitable sense.

1.6 It is crucial to the argument for Theorem B that the Cartan datum is anisotropic. However, there are many other interesting Lie superalgebras which we could consider, most notably those of basic type (cf. [ChW]). For instance, two families of basic type Lie superalgebras are the general linear Lie superalgebra $\mathfrak{g l}(m \mid n)$ and the orthosymplectic Lie superalgebra $\mathfrak{o s p}(m \mid 2 n)$. (The only basic type Lie superalgebras with an anisotropic Cartan datum are $\mathfrak{o s p}(1 \mid 2 n)$ for $n \geq 1$.)

It is an interesting question to ask if Theorem B can be extended to basic type quantum supergroups. This question is partially answered in [CHW3], where canonical bases are constructed for quantum groups associated to $\mathfrak{g l}(1 \mid n), \mathfrak{o s p}(2 \mid 2 n)$, and $\mathfrak{o s p}(1 \mid 2 n)$. However, the methods used are quite different then the ones we shall employ here, and so we shall not discuss these cases. We note that an interesting open question is to relate the bases constructed for $\mathfrak{o s p}(1 \mid 2 n)$ herein and those constructed in loc. cit., and we expect these bases to match.

1.7 The remainder of this dissertation addresses extending the canonical basis to the whole quantum covering group in some sense. In the context of DrinfeldJimbo quantum groups, the precise construction was originally devised in type $A$ in a paper of Beilinson-Lusztig-McPherson [BLM], and generalized by Lusztig [Lu3]. Essentially, we can add orthogonal idempotents to the quantum group associated to each weight with the proviso that the quantum Cartan subalgebra acts on each idempotent according to its weight. The resulting algebra, called a modified quantum group, carries much of the same structure as the quantum group, and has essentially the same representations. This procedure generalizes to quantum covering groups, and so in this way we construct the modified quantum covering group $\dot{\mathbf{U}}$.

Theorem C. Let $\dot{\mathbf{U}}$ be the modified quantum covering group associated to a Cartan 
datum of anisotropic type. Then $\dot{\mathbf{U}}$ admits a canonical basis $\dot{\mathbb{B}}$ of its integral form. Moreover, $\mathbf{U}$ carries a bilinear form $\{-,-\}$, extending the bilinear form on $\mathbf{f}$, which is invariant under several automorphisms of $\dot{\mathbf{U}}$. Moreover, the basis $\dot{\mathbb{B}}$ is almost orthonormal (in a suitable sense) with respect to $\{-,-\}$ on $\dot{\mathbf{U}}$.

Given the prodigious role of such bilinear forms in categorification, we expect our bilinear form on $\mathbf{U}$ to play a significant role in efforts to categorify modified quantum covering groups.

1.8 The following is an outline for the dissertation.

In Chapter 2, we define the notion of an anisotropic Cartan datum and root datum. We then define the algebra $\mathbf{f}$ and deduce some of its important properties and structures. In particular, we define the twistor isomorphism on $\mathbf{f}$ and use it to deduce a Serre presentation for $\mathbf{f}$.

In Chapter 3, we define the quantum covering group. We show that several of the essential properties of quantum groups can be generalized to the covering setting, including a (braided) Hopf algebra structure and a triangular decomposition. We then discuss analogues of weight modules and category $\mathcal{O}$; in particular, we define the Verma modules $M(\lambda)$ and their simple quotient modules $\left.V(\lambda)\right|_{ \pm 1}$. Along the way, we show that the twistor on $\mathbf{f}$ can be extended to an isomorphism on $\mathbf{U}$. Moreover, we show that the Verma and simple modules have induced twistor isomorphisms, and use these to deduce a character formula for the simple modules. We then construct the quasi- $\mathcal{R}$-matrix and a quantum Casimir-type operator, which is then used to prove Theorem A.

In Chapter 4, we develop the machinery for crystal bases. in particular, we define an analogue of Kashiwara's "boson algebra" and use its representation theory to define crystal structure on f. We then introduce a notion of crystal bases for U-modules, a tensor product rule for crystals, and a bilinear form (called a polarization) on each $V(\lambda)$. We then adapt the grand loop argument to the covering setting, proving 
the existence of crystal bases. After developing a relationship between the twistor isomorphism on $\mathbf{f}$ and the crystal structure on $\mathbf{f}$, we show that the crystal bases can be upgraded to the bases in Theorem B.

In Chapter 5, we define the modified form of $\mathbf{U}$ and relate it to the family of U-modules $N\left(\lambda, \lambda^{\prime}\right)$, which are certain tensor products of modules. We define a bar-involution on the $N\left(\lambda, \lambda^{\prime}\right)$ using the quasi- $\mathcal{R}$-matrix (in an analogue to Lusztig's construction) and use it to construct canonical bases for $N\left(\lambda, \lambda^{\prime}\right)$. We then show that these canonical bases can be "glued together" in some sense to a canonical basis of $\dot{\mathbf{U}}$. We then define a bilinear form on $\dot{\mathbf{U}}$ and develop its properties, which proves Theorem C.

Finally, we have an appendix on twisted bialgebras, which are certain generalizations of $\mathbf{f}$. The structure of these algebras are developed with an eye towards understand the twistor isomorphisms and the related constructions in [FL]. In particular, some of these results are used to prove the Serre presentation for $\mathbf{f}$. 


\section{Chapter 2}

\section{The twisted bialgebra $\mathrm{f}$}

\subsection{The Cartan datum}

Definition 2.1. A Cartan datum is a pair $(I, \cdot)$ consisting of a finite set $I$ and a $\mathbb{Z}$-valued symmetric bilinear form $\nu, \nu^{\prime} \mapsto \nu \cdot \nu^{\prime}$ on the free abelian group $\mathbb{Z}[I]$ satisfying

(a) $d_{i}=\frac{i \cdot i}{2} \in \mathbb{Z}_{>0}, \quad \forall i \in I$

(b) $a_{i j}=2 \frac{i \cdot j}{i \cdot i} \in \mathbb{Z}_{\leq 0}$, for $i \neq j$ in $I$.

A Cartan datum is called an anisotropic super Cartan datum (or anisotropic datum, in brief) if there is a partition $I=I_{0} \amalg I_{1}$ which satisfies the anisotropic condition

(c) $2 \frac{i \cdot j}{i \cdot i} \in 2 \mathbb{Z}$ if $i \in I_{1}$.

An anisotropic datum is called bar-consistent if it additionally satisfies

(d) $d_{i} \equiv p(i) \bmod 2, \quad \forall i \in I$.

We will always assume $I_{1} \neq \emptyset$ without loss of generality. An anisotropic datum is always assumed to be bar-consistent in this paper. We note that a bar-consistent anisotropic datum satisfies

$$
i \cdot j \in 2 \mathbb{Z} \quad \text { for all } i, j \in I \text {. }
$$

We set the notation

$$
b_{i j}=1-a_{i j} .
$$


The $i \in I_{0}$ are called even, $i \in I_{1}$ are called odd. We define a parity function $p: I \rightarrow\{0,1\}$ so that $i \in I_{p(i)}$. This function extends to a homomorphism $p: \mathbb{Z}[I] \rightarrow$ $\mathbb{Z} / 2 \mathbb{Z}$, and so $p$ induces a parity $\mathbb{Z} / 2 \mathbb{Z}$-grading on $\mathbb{Z}[I]$.

Remark 2.2. We will freely identify $\mathbb{Z} / 2 \mathbb{Z}$ (and later, $\mathbb{Z} / 4 \mathbb{Z}$ ) with the subset $\{0,1\}$ (resp. $\{0,1,2,3\})$ of $\mathbb{Z}$, and use this identification implicitly in equations; for example, an expression such as $(-1)^{\frac{p(x)^{2}+p(x)}{2}}$ implicitly assumes $p(x) \in\{0,1\}$.

We define the height function on $\mathbb{Z}[I]$ by letting $h t\left(\sum_{i \in I} c_{i} i\right)=\sum_{i \in I} c_{i}$. For $\nu=\sum_{i \in I} \nu_{i} i$, we define the notation

$$
\tilde{\nu}=\sum d_{i} \nu_{i} i
$$

A root datum associated to a anisotropic datum $(I, \cdot)$ consists of

(a) two finitely generated free abelian groups $Y, X$ and a perfect bilinear pairing $\langle-,-\rangle: Y \times X \rightarrow \mathbb{Z}$

(b) an embedding $I \subset X\left(i \mapsto i^{\prime}\right)$ and an embedding $I \subset Y(i \mapsto i)$ satisfying

(c) $\left\langle i, j^{\prime}\right\rangle=\frac{2 i \cdot j}{i \cdot i}$ for all $i, j \in I$.

Remark 2.3. We are using the conventions used by Lusztig in his text [Lu4], which are conveniently minimal for the (sometimes) involved formulae which shall appear. It is simple to pass between this notation and the more traditional root system notation: each $i \in I$ is the index of a simple root $\alpha_{i}$, and the generalized Cartan matrix is given by $a_{i j}$; the embedding $I \subset Y$ gives the dual roots, and the embedding $I \subset X$ realizes the simple roots as elements of the weight lattice.

If the image of the imbedding $I \subset X$ (respectively, the image of the imbedding $I \subset Y$ ) is linearly independent in $X$ (respectively, in $Y$ ), then we say that the root datum is $X$-regular (resp. $Y$-regular).

For $i \neq j \in I$ such that $\left\langle i, j^{\prime}\right\rangle\left\langle j, i^{\prime}\right\rangle>0$, we define an integer $m_{i j} \geq 2$ by $\cos ^{2} \frac{\pi}{m_{i j}}=\frac{1}{4}\left\langle i, j^{\prime}\right\rangle\left\langle j, i^{\prime}\right\rangle$ if it exists, and set $m_{i j}=\infty$ otherwise. We have 


$$
\begin{array}{c|ccccc}
\left\langle i, j^{\prime}\right\rangle\left\langle j, i^{\prime}\right\rangle & 0 & 1 & 2 & 3 & \geq 4 \\
\hline m_{i j} & 2 & 3 & 4 & 6 & \infty
\end{array}
$$

The braid group (associated to $I$ ) is the group generated by $s_{i}(i \in I)$ subject to the relations (whenever $\left.m_{i j}<\infty\right)$ :

$$
\underbrace{s_{i} s_{j} s_{i} \cdots}_{m_{i j}}=\underbrace{s_{j} s_{i} s_{j} \cdots}_{m_{i j}}
$$

The Weyl group $W$ is defined to be the group generated by $s_{i}(i \in I)$ subject to relations (2.4) and additional relations $s_{i}^{2}=1$ for all $i$.

For $i \in I$, we let $s_{i}$ act on $X$ (resp. $Y$ ) as follows: for $\lambda \in X, \lambda^{\vee} \in Y$,

$$
s_{i}(\lambda)=\lambda-\langle i, \lambda\rangle i^{\prime}, \quad s_{i}\left(\lambda^{\vee}\right)=\lambda^{\vee}-\left\langle\lambda^{\vee}, i^{\prime}\right\rangle i
$$

This defines actions of the Weyl group $W$ on $X$ and $Y$.

If $V$ is a vector space graded by $\mathbb{Z}[I]$ (respectively, $X$ ), we will use the weight notation $|x|=\mu$ if $x \in V_{\mu}$ for $\mu \in \mathbb{Z}[I]$ (respectively, $X$ ). If $V$ is a $\mathbb{Z} / 2 \mathbb{Z}$-graded vector space, we will use the parity notation $p(x)=a$ if $x \in V_{a}$ for $a \in \mathbb{Z} / 2 \mathbb{Z}$. In particular, all formulae containing the notations $|\cdot|$ and $p(\cdot)$ implicitly assume that the elements within are homogeneous with respect to the corresponding grading.

\subsection{Quantum parameters}

Let $q$ be a formal parameter and let $\pi$ be an indeterminate such that

$$
\pi^{2}=1
$$

For a ring $R$ with 1 , we will form a new $\operatorname{ring} R^{\pi}=R[\pi] /\left(\pi^{2}-1\right)$. Given an $R^{\pi}$-module (or algebra) $M$, the specialization of $M$ at $\pi= \pm 1$ means the $R$-module (or algebra) $\left.M\right|_{\pi= \pm 1}=R_{ \pm} \otimes_{R^{\pi}} M$, where $R_{ \pm}=R$ is viewed as a $R^{\pi}$-module on which $\pi$ acts as \pm 1 . 
Assume 2 is invertible in $R$; i.e. $\frac{1}{2} \in R$. We define

$$
\varepsilon_{+}=\frac{1+\pi}{2}, \quad \varepsilon_{-}=\frac{1-\pi}{2}
$$

and note that $R^{\pi}=R \varepsilon_{+} \oplus R \varepsilon_{-}$. In particular, since $\pi \varepsilon_{ \pm}= \pm \varepsilon_{ \pm}$for an $R^{\pi}$-module $M$, we see that

$$
\left.M\right|_{\pi= \pm 1} \cong \varepsilon_{ \pm 1} M
$$

The principle rings of concern in this paper are $\mathbb{Q}(q)^{\pi}$ and $\mathbb{A}=\mathbb{Z}\left[q, q^{-1}\right]^{\pi}$. Unless otherwise stated, by convention the tensor product of $\mathbb{Q}(q)^{\pi}$-modules is always taken over $\mathbb{Q}(q)^{\pi}$.

For $k \in \mathbb{Z}_{\geq 0}$ and $n \in \mathbb{Z}$, we use a $(q, \pi)$-variant of quantum integers, quantum factorial and quantum binomial coefficients:

$$
\begin{aligned}
& {[n]_{q, \pi} }=\frac{(\pi q)^{n}-q^{-n}}{\pi q-q^{-1}} \in \mathbb{A}, \\
& {[n]_{q, \pi}^{!} }=\prod_{l=1}^{n}[l]_{q, \pi} \in \mathbb{A}, \\
& {\left[\begin{array}{l}
n \\
k
\end{array}\right]_{q, \pi}=\frac{\prod_{l=n-k+1}^{n}\left((\pi q)^{l}-q^{-l}\right)}{\prod_{m=1}^{k}\left((\pi q)^{m}-q^{-m}\right)} \in \mathbb{A} . }
\end{aligned}
$$

These binomial coefficients arise naturally in the following way. If $x, y$ are two elements in a $\mathbb{Q}(q)^{\pi}$-algebra such that $x y=\pi q^{2} y x$, then for any $a \geq 0$, we have the quantum binomial formula:

$$
(x+y)^{a}=\sum_{t=0}^{a} q^{t(a-t)}\left[\begin{array}{l}
a \\
t
\end{array}\right]_{q, \pi} y^{t} x^{a-t} .
$$

We will use the notation

$$
q_{i}=q^{d_{i}}, \quad \pi_{i}=\pi^{d_{i}}, \quad \text { for } i \in I .
$$

More generally, for $\nu=\sum \nu_{i} i$, we set

$$
q_{\nu}=\prod_{i \in I} q_{i}^{\nu_{i}}, \quad \pi_{\nu}=\prod_{i \in I} \pi_{i}^{\nu_{i}}
$$


There are two notable automorphisms of $\mathbb{Q}(q)^{\pi}$ and $\mathbb{A}$ for our purposes. The bar involution on $\mathbb{Q}(q)^{\pi}$ is the $\mathbb{Q}^{\pi}$-algebra automorphism defined by $\overline{f(q)}=f\left(\pi q^{-1}\right)$ for $f(q) \in \mathbb{Q}(q)^{\pi}$. The dagger involution on $\mathbb{Q}(q)^{\pi}$ is the $\mathbb{Q}^{\pi}$-algebra automorphism defined by $f(q)^{\dagger}=f(\pi q)$ for $f(q) \in \mathbb{Q}(q)^{\pi}$. We note that both involutions restict to $\mathbb{Z}^{\pi}$-algebra automorphisms of $\mathbb{A}$.

Note that the $(q, \pi)$-integers $[n]_{i}$ and the $(q, \pi)$-binomial coefficients in general are not necessarily bar-invariant unless the anisotropic Cartan datum is bar-consistent. Moreover, the $(q, \pi)$ integers are not $\dagger$-invariant in general; to wit,

$$
[k]_{q, \pi}^{\dagger}=\pi^{k-1}[k]_{q, \pi}, \quad\left([k]_{q, \pi}^{!}\right)^{\dagger}=\pi^{\left(\begin{array}{c}
k \\
2
\end{array}\right)}[k]_{q, \pi}^{!}, \quad\left[\begin{array}{c}
n \\
k
\end{array}\right]_{q, \pi}^{\dagger}=\pi^{k(n-k)}\left[\begin{array}{c}
n \\
k
\end{array}\right]_{q, \pi}
$$

In particular, we note that $\left[\begin{array}{l}n \\ k\end{array}\right]_{q, \pi}$ is $\dagger$-invariant if and only if $n$ is odd or $k$ is even.

Remark 2.4. We note that $[n]^{\dagger}=\frac{q^{n}-(\pi q)^{-n}}{q-\pi q^{-1}}$ could well be the definition of $(q, \pi)$ integers, and we regard it as an alternate convention.

These $(q, \pi)$-quantum integers satisfy identities analogous to more traditional quantum integers.

$$
\begin{aligned}
& {\left[\begin{array}{l}
a \\
t
\end{array}\right]_{q, \pi}=(-1)^{t} \pi^{t a-\left(\begin{array}{c}
t \\
2
\end{array}\right)}\left[\begin{array}{c}
t-a-1 \\
t
\end{array}\right]_{q, \pi},} \\
& {\left[\begin{array}{l}
a \\
t
\end{array}\right]_{q, \pi}=\left\{\begin{array}{ll}
\frac{[a]_{q, \pi}^{!}}{[t]_{q, \pi}^{!}[a-t]_{q, \pi}^{!}} & \text {if } 0 \leq t \leq a \\
0 & \text { if } a>t
\end{array} \quad \text { if } a \geq 0\right.} \\
& \prod_{j=0}^{a-1}\left(1+\left(\pi q^{2}\right)^{j} z\right)=\sum_{t=0}^{a} \pi^{\left(\begin{array}{l}
t \\
2
\end{array}\right)} q^{t(a-1)}\left[\begin{array}{l}
a \\
t
\end{array}\right]_{q, \pi} z^{t} \quad \text { if } a \geq 0
\end{aligned}
$$

Here $z$ is another indeterminate. If $a^{\prime}, a^{\prime \prime}$ are integers and $t \in \mathbb{N}$, then

$$
\left[\begin{array}{c}
a^{\prime}+a^{\prime \prime} \\
t
\end{array}\right]_{q, \pi}=\sum_{t^{\prime}+t^{\prime \prime}=t} \pi_{i}^{t^{\prime} t^{\prime \prime}+a^{\prime} t^{\prime \prime}} q_{i}^{a^{\prime} t^{\prime \prime}-a^{\prime \prime} t^{\prime}}\left[\begin{array}{l}
a^{\prime} \\
t^{\prime}
\end{array}\right]_{q, \pi}\left[\begin{array}{l}
a^{\prime \prime} \\
t^{\prime \prime}
\end{array}\right]_{q, \pi}
$$

These identities can be proved directly, or by specializing analogous identities in the Gaussian integers $\langle n\rangle_{x}=\frac{1-x^{n}}{1-x}$ at $x=\pi q^{2}$. This latter strategy is demonstrated 
in [CHW2, Lemma 7.2] which proves the following identity: if $r \geq 0$ and $n \geq 1$, then

$$
\sum_{t=0}^{r}(-1)^{t} \pi^{(t+n)(r+n)+\left(\begin{array}{c}
t+n+1 \\
2
\end{array}\right)}\left[\begin{array}{c}
t+n-1 \\
t
\end{array}\right]_{q, \pi}\left[\begin{array}{c}
t+r+n \\
r
\end{array}\right]_{q, \pi}\left[\begin{array}{c}
r+n \\
t+n
\end{array}\right]_{q, \pi}=\pi^{\left(\begin{array}{c}
n \\
2
\end{array}\right)} .
$$

We note the following specializations of some of the above identities. Observe that $\left[\begin{array}{c}-1 \\ t\end{array}\right]_{q, \pi}=(-1)^{t} \pi^{\left(\begin{array}{c}t+1 \\ 2\end{array}\right)}$ for any $t \geq 0, i \in I$. Furthermore if $a \geq 1$, then we have

$$
\sum_{t=0}^{a}(-1)^{t} \pi^{\left(\begin{array}{l}
t \\
2
\end{array}\right)} q^{t(a-1)}\left[\begin{array}{l}
a \\
t
\end{array}\right]_{q, \pi}=0
$$

which follows from (2.10) by setting $z=-1$.

\subsection{The algebras 'f and $f$}

We define 'f to be the associative $\mathbb{Q}(q)^{\pi}$-algebra with 1 and with generators $\theta_{i}$ for $i \in I$. The algebra 'f $\mathbf{f}$ is a superalgebra, where the parity grading on ' $\mathbf{f}$ is given by $p\left(\theta_{i}\right)=p(i)$. We also have a $\mathbb{N}[I]$-grading $|\cdot|$ on 'f defined by setting $\left|\theta_{i}\right|=i$.

The tensor product 'f $\otimes$ 'f can be equipped with an associative $\mathbb{Q}(q)^{\pi}$-algebra structure with multiplication defined by

$$
\left(x_{1} \otimes x_{2}\right)\left(x_{1}^{\prime} \otimes x_{2}^{\prime}\right)=q^{\left|x_{2}\right| \cdot\left|x_{1}^{\prime}\right|} \pi^{p\left(x_{2}\right) p\left(x_{1}^{\prime}\right)} x_{1} x_{1}^{\prime} \otimes x_{2} x_{2}^{\prime}
$$

This structure can be extended to arbitrary tensor powers of 'f algebra 'f $\otimes^{\prime} \mathbf{f} \otimes^{\prime} \mathbf{f}$, has an associative product defined by:

$$
\begin{aligned}
& \left(x_{1} \otimes x_{2} \otimes x_{3}\right)\left(x_{1}^{\prime} \otimes x_{2}^{\prime} \otimes x_{3}^{\prime}\right) \\
& \quad=q^{\left|x_{2}\right| \cdot\left|x_{1}^{\prime}\right|+\left|x_{3}\right| \cdot\left|x_{2}^{\prime}\right|+\left|x_{3}\right| \cdot\left|x_{1}^{\prime}\right|} \pi^{p\left(x_{2}\right) p\left(x_{1}^{\prime}\right)+p\left(x_{3}\right) p\left(x_{2}^{\prime}\right)+p\left(x_{3}\right) p\left(x_{1}^{\prime}\right)} x_{1} x_{1}^{\prime} \otimes x_{2} x_{2}^{\prime} \otimes x_{3} x_{3}^{\prime} .
\end{aligned}
$$

We define the coproduct $r:{ }^{\prime} \mathbf{f} \rightarrow{ }^{\prime} \mathbf{f} \otimes$ 'f to be the algebra homomorphism satisfying $r\left(\theta_{i}\right)=\theta_{i} \otimes 1+1 \otimes \theta_{i}$ for all $i \in I$. One checks the following equality of algebra homomorphisms holds:

$$
(r \otimes 1) r=(1 \otimes r) r:{ }^{\prime} \mathbf{f} \rightarrow{ }^{\prime} \mathbf{f} \otimes^{\prime} \mathbf{f} \otimes^{\prime} \mathbf{f} .
$$


In particular, there are well defined homomorphisms $r^{n}:{ }^{\prime} \mathbf{f} \rightarrow^{\prime} \mathbf{f}^{\otimes(n+1)}$ for all $n \geq 1$ obtained by inductively applying $r$ to any choice of tensor factor. The coproduct allows us to construct a bilinear form as follows.

Proposition 2.5. There exists a unique bilinear form $(-,-)$ on ' $\mathbf{f}$ with values in $\mathbb{Q}$ such that $(1,1)=1$ and

(a) $\left(\theta_{i}, \theta_{j}\right)=\delta_{i j}\left(1-\pi_{i} q_{i}^{-2}\right)^{-1} \quad(\forall i, j \in I)$;

(b) $\left(x, y^{\prime} y^{\prime \prime}\right)=\left(r(x), y^{\prime} \otimes y^{\prime \prime}\right) \quad\left(\forall x, y^{\prime}, y^{\prime \prime} \in{ }^{\prime} \mathbf{f}\right)$;

(c) $\left(x x^{\prime}, y^{\prime \prime}\right)=\left(x \otimes x^{\prime}, r\left(y^{\prime \prime}\right)\right) \quad\left(\forall x, x^{\prime}, y^{\prime \prime} \in{ }^{\prime} \mathbf{f}\right)$.

Moreover, this bilinear form is symmetric.

Here, the induced bilinear form $\left({ }^{\prime} \mathbf{f} \otimes{ }^{\prime} \mathbf{f}\right) \times\left({ }^{\prime} \mathbf{f} \otimes^{\prime} \mathbf{f}\right) \rightarrow \mathbb{Q}(q)$ is given by

$$
\left(x_{1} \otimes x_{2}, x_{1}^{\prime} \otimes x_{2}^{\prime}\right):=\left(x_{1}, x_{1}^{\prime}\right)\left(x_{2}, x_{2}^{\prime}\right)
$$

for homogeneous $x_{1}, x_{2}, x_{1}^{\prime}, x_{2}^{\prime} \in$ 'f.

Proof. Define an associative algebra structure on ' $\mathbf{f}^{*}:=\oplus_{\nu}{ }^{\prime} \mathbf{f}_{\nu}^{*}$ by transposing the "coproduct" $r:$ 'f $\rightarrow$ 'f $\otimes$ 'f. In particular, for $g, h \in{ }^{\prime} \mathbf{f}^{*}$, we define $g h(x):=$ $(g \otimes h)(r(x))$, where $(g \otimes h)(y \otimes z)=g(y) h(z)$.

Let $\xi_{i} \in$ 'f $_{i}^{*}$ be defined by $\xi_{i}\left(\theta_{i}\right)=\left(1-\pi_{i} q_{i}^{-2}\right)^{-1}$. Let $\phi: ' \mathbf{f} \rightarrow{ }^{\prime} \mathbf{f}^{*}$ be the unique algebra homomorphism such that $\phi\left(\theta_{i}\right)=\xi_{i}$ for all $i$. The map $\phi$ preserves the $\mathbb{N}[I] \times \mathbb{Z}_{2}$-grading.

Define $(x, y)=\phi(y)(x)$, for $x, y \in$ 'f. The properties (a) and (b) follow directly from the definition. Clearly $(x, y)=0$ unless (homogeneous) $x, y$ have the same weight in $\mathbb{N}[I]$ and the same parity. All elements involved below will be assumed to be homogeneous. 
It remains to prove (c). Assume that (c) is known for $y^{\prime \prime}$ replaced by $y$ or $y^{\prime}$ and for any $x, x^{\prime}$. We then prove that (c) holds for $y^{\prime \prime}=y y^{\prime}$. Write

$$
\begin{aligned}
& r(x)=\sum x_{1} \otimes x_{2}, \quad r\left(x^{\prime}\right)=\sum x_{1}^{\prime} \otimes x_{2}^{\prime}, \\
& r(y)=\sum y_{1} \otimes y_{2}, \quad r\left(y^{\prime}\right)=\sum y_{1}^{\prime} \otimes y_{2}^{\prime} .
\end{aligned}
$$

Then

$$
\begin{aligned}
& r\left(x x^{\prime}\right)=\sum q^{\left|x_{2}\right| \cdot\left|x_{1}^{\prime}\right|} \pi^{p\left(x_{2}\right) p\left(x_{1}^{\prime}\right)} x_{1} x_{1}^{\prime} \otimes x_{2} x_{2}^{\prime}, \\
& r\left(y y^{\prime}\right)=\sum q^{\left|y_{2}\right| \cdot\left|y_{1}^{\prime}\right|} \pi^{p\left(y_{2}\right) p\left(y_{1}^{\prime}\right)} y_{1} y_{1}^{\prime} \otimes y_{2} y_{2}^{\prime} .
\end{aligned}
$$

We have

$$
\begin{aligned}
& \left(x x^{\prime}, y y^{\prime}\right)=\left(\phi(y) \phi\left(y^{\prime}\right)\right)\left(x x^{\prime}\right)=\left(\phi(y) \otimes \phi\left(y^{\prime}\right)\right)\left(r\left(x x^{\prime}\right)\right) \\
& =\sum q^{\left|x_{2}\right| \cdot\left|x_{1}^{\prime}\right|} \pi^{p\left(x_{2}\right) p\left(x_{1}^{\prime}\right)}\left(x_{1} x_{1}^{\prime}, y\right)\left(x_{2} x_{2}^{\prime}, y^{\prime}\right) \\
& =\sum q^{\left|x_{2}\right| \cdot\left|x_{1}^{\prime}\right|} \pi^{p\left(x_{2}\right) p\left(x_{1}^{\prime}\right)}\left(x_{1} \otimes x_{1}^{\prime}, r(y)\right)\left(x_{2} \otimes x_{2}^{\prime}, r\left(y^{\prime}\right)\right) \\
& =\sum q^{\left|x_{2}\right| \cdot\left|x_{1}^{\prime}\right|} \pi^{p\left(x_{2}\right) p\left(x_{1}^{\prime}\right)}\left(x_{1}, y_{1}\right)\left(x_{1}^{\prime}, y_{2}\right)\left(x_{2}, y_{1}^{\prime}\right)\left(x_{2}^{\prime}, y_{2}^{\prime}\right) .
\end{aligned}
$$

On the other hand,

$$
\begin{aligned}
& \left(x \otimes x^{\prime}, r\left(y y^{\prime}\right)\right)=\sum q^{\left|y_{2}\right| \cdot\left|y_{1}^{\prime}\right|} \pi^{p\left(y_{2}\right) p\left(y_{1}^{\prime}\right)}\left(x \otimes x^{\prime}, y_{1} y_{1}^{\prime} \otimes y_{2} y_{2}^{\prime}\right) \\
& =\sum q^{\left|y_{2}\right| \cdot\left|y_{1}^{\prime}\right|} \pi^{p\left(y_{2}\right) p\left(y_{1}^{\prime}\right)}\left(x, y_{1} y_{1}^{\prime}\right)\left(x^{\prime}, y_{2} y_{2}^{\prime}\right) \\
& =\sum q^{\left|y_{2}\right| \cdot\left|y_{1}^{\prime}\right|} \pi^{p\left(y_{2}\right) p\left(y_{1}^{\prime}\right)}\left(r(x), y_{1} \otimes y_{1}^{\prime}\right)\left(r\left(x^{\prime}\right), y_{2} \otimes y_{2}^{\prime}\right) \\
& =\sum q^{\left|y_{2}\right| \cdot\left|y_{1}^{\prime}\right|} \pi^{p\left(y_{2}\right) p\left(y_{1}^{\prime}\right)}\left(x_{1}, y_{1}\right)\left(x_{1}^{\prime}, y_{2}\right)\left(x_{2}, y_{1}^{\prime}\right)\left(x_{2}^{\prime}, y_{2}^{\prime}\right) .
\end{aligned}
$$

For a summand to make nonzero contribution, we may assume that each of the four pairs $\left\{x_{1}, y_{1}\right\},\left\{x_{1}^{\prime}, y_{2}\right\},\left\{x_{2}, y_{1}^{\prime}\right\},\left\{x_{2}^{\prime}, y_{2}^{\prime}\right\}$ have the same weight in $\mathbb{N}[I]$ and the same parity. One checks that the powers of $q$ and $\pi$ in (2.15) and (2.16) match perfectly. Hence the two sums in (2.15) and (2.16) are equal, and whence (c).

We set $\mathcal{I}$ to denote the radical of $(-,-)$. As in [Lu4], this radical is a 2 -sided ideal of 'f. Let $\mathbf{f}={ }^{\prime} \mathbf{f} / \mathcal{I}$ be the quotient algebra of 'f by its radical. Since the different 
weight spaces are orthogonal with respect to this inner product, the weight space decomposition descends to a decomposition $\mathbf{f}=\bigoplus_{\nu} \mathbf{f}_{\nu}$ where $\mathbf{f}_{\nu}$ is the image of ${ }^{\prime} \mathbf{f}_{\nu}$. Each weight space is finite dimensional. The bilinear form descends to a bilinear form on $\mathbf{f}$ which is non-degenerate on each weight space.

Remark 2.6. An alternate convention for extending the inner product to ' $\mathbf{f}^{\otimes 2}$ which we could take is

$$
\left(x_{1} \otimes x_{2}, x_{1}^{\prime} \otimes x_{2}^{\prime}\right)_{\text {signed }}:=\pi^{p\left(x_{1}^{\prime}\right) p\left(x_{2}\right)}\left(x_{1}, x_{1}^{\prime}\right)\left(x_{2}, x_{2}^{\prime}\right) .
$$

For example, this is the convention taken in $[Y 1, G e]$. We note that $(-,-)_{\text {signed }}$ is equivalent to $(-,-)$ up to a renormalization on each weight space; see Corollary A.8 or [CHW3, Lemma 2.3]. In particular, the radicals are identical so there is no ambiguity in the definition of $\mathbf{f}$.

The map $r: ' \mathbf{f} \rightarrow{ }^{\prime} \mathbf{f}^{\otimes 2}$ satisfies $r(\mathcal{I}) \subset \mathcal{I} \otimes \mathbf{f}^{\prime}{ }^{\prime} \mathbf{f} \otimes \mathcal{I}$, essentially by construction, whence it descends to a well-defined homomorphism $r: \mathbf{f} \rightarrow \mathbf{f}^{\otimes 2}$. Let ${ }^{t} r:{ }^{\prime} \mathbf{f} \rightarrow{ }^{\prime} \mathbf{f}^{\otimes 2}$ be the composition of $r$ with the permutation map

$$
x \otimes y \mapsto y \otimes x
$$

of ${ }^{\prime} \mathbf{f}^{\otimes 2}$ to itself. (Note that this map is an anti-automorphism of ${ }^{\prime} \mathbf{f}^{\otimes 2}$.) We also let $\varrho: ' \mathbf{f} \rightarrow$ 'f be the map satisfying $\varrho\left(\theta_{i}\right)=\theta_{i}$ for each $i \in I$ and

$$
\varrho(x y)=\varrho(y) \varrho(x) .
$$

Lemma 2.7. (a) We have $r(\varrho(x))=(\varrho \otimes \varrho)^{t} r(x)$, for all $x \in$ 'f.

(b) We have $\left(\varrho(x), \varrho\left(x^{\prime}\right)\right)=\left(x, x^{\prime}\right)$ for all $x, x^{\prime} \in$ 'f.

(c) @ descends to $\mathbf{f}$ and satisfies (a) and (b).

Proof. First note that (c) follows from the fact that (b) implies $\varrho(\mathcal{I}) \subset \mathcal{I}$. Since (b) will follow immediately from (a), it suffices to prove that $r(\varrho(x))=(\varrho \otimes \varrho)^{t} r(x)$, for all $x \in$ 'f. This is obviously true for $x \in\left\{1, \theta_{i}: i \in I\right\}$. 
Suppose that $r\left(\varrho\left(x^{\prime}\right)\right)=(\varrho \otimes \varrho)^{t} r\left(x^{\prime}\right)$ and $r\left(\varrho\left(x^{\prime \prime}\right)\right)=(\varrho \otimes \varrho)^{t} r\left(x^{\prime \prime}\right)$. Let $r\left(x^{\prime}\right)=$ $\sum x_{1}^{\prime} \otimes x_{2}^{\prime}$ and $r\left(x^{\prime \prime}\right)=\sum x_{1}^{\prime \prime} \otimes x_{2}^{\prime \prime}$. Then $r\left(x^{\prime} x^{\prime \prime}\right)=\sum q^{\left|x_{2}^{\prime}\right|\left|x_{1}^{\prime \prime}\right|} \pi^{p\left(x_{2}^{\prime}\right) p\left(x_{1}^{\prime \prime}\right)} x_{1}^{\prime} x_{1}^{\prime \prime} \otimes x_{2}^{\prime} x_{2}^{\prime \prime}$ and we have

$$
\begin{aligned}
r\left(\varrho\left(x^{\prime} x^{\prime \prime}\right)\right) & =r\left(\varrho\left(x^{\prime \prime}\right)\right) r\left(\varrho\left(x^{\prime}\right)\right) \\
& =\left(\sum \varrho\left(x_{2}^{\prime \prime}\right) \otimes \varrho\left(x_{1}^{\prime \prime}\right)\right)\left(\sum \varrho\left(x_{2}^{\prime}\right) \otimes \varrho\left(x_{1}^{\prime}\right)\right) \\
& =\sum \pi^{p\left(x_{2}^{\prime}\right) p\left(x_{1}^{\prime \prime}\right)} q^{\left|x_{2}^{\prime}\right|\left|x_{1}^{\prime \prime}\right|} \varrho\left(x_{2}^{\prime} x_{2}^{\prime \prime}\right) \otimes \varrho\left(x_{1}^{\prime} x_{1}^{\prime \prime}\right)=\varrho \otimes \varrho\left({ }^{t} r\left(x^{\prime} x^{\prime \prime}\right)\right) .
\end{aligned}
$$

The lemma is proved.

We define a bar involution ${ }^{-}:$' $\mathbf{f} \rightarrow$ ' $^{\mathbf{f}}$ such that $\overline{\theta_{i}}=\theta_{i}$ for all $i \in I$ and $\overline{f x}=\bar{f} \bar{x}$ for

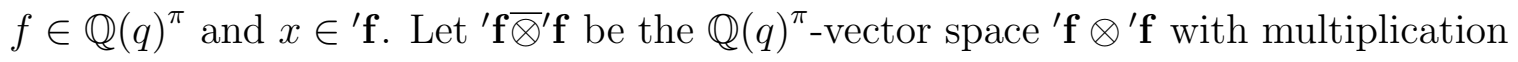
given by

$$
\left(x_{1} \otimes x_{2}\right)\left(x_{1}^{\prime} \otimes x_{2}^{\prime}\right)=\left(\pi q^{-1}\right)^{\left|x_{2}\right| \cdot\left|x_{1}^{\prime}\right|} \pi^{p\left(x_{2}\right) p\left(x_{1}^{\prime}\right)} x_{1} x_{1}^{\prime} \otimes x_{2} x_{2}^{\prime} .
$$

Define $\bar{r}$ still by $\bar{r}(x)=\overline{r(\bar{x})}$. Then $\bar{r}:$ ' $\mathbf{f} \rightarrow{ }^{\prime} \mathbf{f} \bar{\otimes}$ ' $\mathbf{f}$ is an algebra homomorphism, being a composition of homomorphisms. The co-associativity holds for $\bar{r}$ :

$$
(\bar{r} \otimes 1)(\bar{r}(x))=\overline{(r \otimes 1) r(\bar{x})}=\overline{(1 \otimes r) r(\bar{x})}=(1 \otimes \bar{r})(\bar{r}(x)) .
$$

By checking on the algebra generators $\theta_{i}$, it is an easy computation to see that this is an algebra homomorphism.

Let $\{-,-\}: ' \mathbf{f} \times$ ' $\mathbf{f} \rightarrow \mathbb{Q}(q)$ be the symmetric bilinear form defined by

$$
\{x, y\}=\overline{(\bar{x}, \bar{y})} .
$$

Then we have $\{1,1\}=1,\left\{\theta_{i}, \theta_{j}\right\}=\delta_{i, j}\left(1-\pi_{i} q_{i}^{2}\right)^{-1}$, and

$$
\left\{x, y^{\prime} y^{\prime \prime}\right\}=\left\{\bar{r}(x), y^{\prime} \otimes y^{\prime \prime}\right\}, \text { for all } x, y^{\prime}, y^{\prime \prime} \in{ }^{\prime} \mathbf{f} .
$$

Lemma 2.8. (a) Let $r(x)=\sum x_{1} \otimes x_{2}$. We have

$$
\bar{r}(x)=\sum(\pi q)^{-\left|x_{1}\right| \cdot\left|x_{2}\right|} \pi^{p\left(x_{1}\right) p\left(x_{2}\right)} x_{2} \otimes x_{1} .
$$


(b) $\{x, y\}=(-1)^{\mathrm{ht}|x|} \pi^{\frac{p(x) p(y)+p(x)}{2}} q^{-|x| \cdot|y| / 2} q_{-|x|}(x, \varrho(y))$.

(c) - descends to an involution on $\mathbf{f}$.

Proof. It is straightforward to check both claims are true when $x=\theta_{i}$ and $y=\theta_{j}$ for some $i, j \in I$. Assume (a) holds for $x$ replaced by $x^{\prime}$ and by $x^{\prime \prime}$. We shall prove the claim for $x=x^{\prime} x^{\prime \prime}$. Recall $\bar{q}=\pi q^{-1}$, and $r(\bar{x})=\overline{\bar{r}(x)}$. Write

$$
\begin{aligned}
r\left(x^{\prime}\right) & =\sum x_{1}^{\prime} \otimes x_{2}^{\prime}, \quad r\left(x^{\prime \prime}\right)=\sum x_{1}^{\prime \prime} \otimes x_{2}^{\prime \prime}, \\
r\left(x^{\prime} x^{\prime \prime}\right) & =\sum q^{\left|x_{1}^{\prime \prime}\right| \cdot x_{2}^{\prime} \mid} \pi^{p\left(x_{1}^{\prime \prime}\right) p\left(x_{2}^{\prime}\right)} x_{1}^{\prime} x_{1}^{\prime \prime} \otimes x_{2}^{\prime} x_{2}^{\prime \prime} .
\end{aligned}
$$

By assumption, we have

$$
\begin{aligned}
& r\left(\overline{x^{\prime}}\right)=\sum q^{\left|x_{1}^{\prime}\right| \cdot\left|x_{2}^{\prime}\right|} \pi^{p\left(x_{1}^{\prime}\right) p\left(x_{2}^{\prime}\right)} \overline{x_{2}^{\prime}} \otimes \overline{x_{1}^{\prime}} \\
& r\left(\overline{x^{\prime \prime}}\right)=\sum q^{\left|x_{1}^{\prime \prime}\right| \cdot\left|x_{2}^{\prime \prime}\right|} \pi^{p\left(x_{1}^{\prime \prime}\right) p\left(x_{2}^{\prime \prime}\right)} \overline{x_{2}^{\prime \prime}} \otimes \overline{x_{1}^{\prime \prime}}
\end{aligned}
$$

Hence,

$$
\begin{aligned}
r\left(\overline{x^{\prime}}\right) r\left(\overline{x^{\prime \prime}}\right) & =\sum q^{\left|x_{1}^{\prime}\right| \cdot\left|x_{2}^{\prime}\right|} \pi^{p\left(x_{1}^{\prime}\right) p\left(x_{2}^{\prime}\right)} q^{\left|x_{1}^{\prime \prime}\right| \cdot\left|x_{2}^{\prime \prime}\right|} \pi^{p\left(x_{1}^{\prime \prime}\right) p\left(x_{2}^{\prime \prime}\right)}\left(\overline{x_{2}^{\prime}} \otimes \overline{x_{1}^{\prime}}\right)\left(\overline{x_{2}^{\prime \prime}} \otimes \overline{x_{1}^{\prime \prime}}\right) \\
& =\sum q^{\left|x_{1}^{\prime}\right| \cdot\left|x_{2}^{\prime}\right|+\left|x_{1}^{\prime \prime}\right| \cdot\left|x_{2}^{\prime \prime}\right|} \pi^{p\left(x_{1}^{\prime}\right) p\left(x_{2}^{\prime}\right)+p\left(x_{1}^{\prime \prime}\right) p\left(x_{2}^{\prime \prime}\right)+p\left(x_{1}^{\prime}\right) p\left(x_{2}^{\prime \prime}\right)} q^{\left|x_{1}^{\prime}\right| \cdot\left|x_{2}^{\prime \prime}\right|} \overline{x_{2}^{\prime} x_{2}^{\prime \prime}} \otimes \overline{x_{1}^{\prime} x_{1}^{\prime \prime}}
\end{aligned}
$$

Then,

$$
\begin{aligned}
\bar{r}\left(x^{\prime} x^{\prime \prime}\right) & =\overline{r\left(\overline{x^{\prime}}\right) r\left(\overline{x^{\prime \prime}}\right)} \\
& =\sum(\pi q)^{-\left(\left|x_{1}^{\prime}\right| \cdot\left|x_{2}^{\prime}\right|+\left|x_{1}^{\prime \prime}\right| \cdot\left|x_{2}^{\prime \prime}\right|+\left|x_{1}^{\prime}\right| \cdot\left|x_{2}^{\prime \prime}\right|\right)} \pi^{p\left(x_{1}^{\prime}\right) p\left(x_{2}^{\prime}\right)+p\left(x_{1}^{\prime \prime}\right) p\left(x_{2}^{\prime \prime}\right)+p\left(x_{1}^{\prime}\right) p\left(x_{2}^{\prime \prime}\right)} x_{2}^{\prime} x_{2}^{\prime \prime} \otimes x_{1}^{\prime} x_{1}^{\prime \prime} \\
& =\sum(\pi q)^{-\left|x_{1}^{\prime} x_{1}^{\prime \prime}\right| \cdot\left|x_{2}^{\prime} x_{2}^{\prime \prime}\right|} \pi^{p\left(x_{1}^{\prime} x_{1}^{\prime \prime}\right) p\left(x_{2}^{\prime} x_{2}^{\prime \prime}\right)} q^{\left|x_{1}^{\prime \prime}\right| \cdot\left|x_{2}^{\prime}\right|} \pi^{p\left(x_{1}^{\prime \prime}\right) p\left(x_{2}^{\prime}\right)+\left|x_{1}^{\prime \prime}\right| \cdot\left|x_{2}^{\prime}\right|} x_{2}^{\prime} x_{2}^{\prime \prime} \otimes x_{1}^{\prime} x_{1}^{\prime \prime} .
\end{aligned}
$$

Now, since the datum is consistent, $\left|x_{1}^{\prime \prime}\right| \cdot\left|x_{2}^{\prime}\right| \in 2 \mathbb{Z}$, and hence we have

$$
\bar{r}\left(x^{\prime} x^{\prime \prime}\right)=\sum(\pi q)^{-\left|x_{1}^{\prime} x_{1}^{\prime \prime}\right| \cdot\left|x_{2}^{\prime} x_{2}^{\prime \prime}\right|} \pi^{p\left(x_{1}^{\prime} x_{1}^{\prime \prime}\right) p\left(x_{2}^{\prime} x_{2}^{\prime \prime}\right)} q^{\left|x_{1}^{\prime \prime}\right| \cdot\left|x_{2}^{\prime}\right|} \pi^{p\left(x_{1}^{\prime \prime}\right) p\left(x_{2}^{\prime}\right)} x_{2}^{\prime} x_{2}^{\prime \prime} \otimes x_{1}^{\prime} x_{1}^{\prime \prime}
$$

Comparing (2.17) and (2.18), we see that (a) holds. 


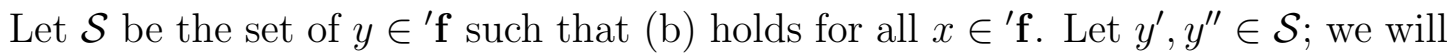
show $y=y^{\prime} y^{\prime \prime} \in \mathcal{S}$ Let $x \in$ 'f $^{\prime}$ and write $r(x)=\sum x^{\prime} \otimes x^{\prime \prime}$ with $x, x^{\prime \prime}$ homogeneous. Then

$$
\begin{aligned}
\left\{x, y^{\prime} y^{\prime \prime}\right\}= & \left\{\bar{r}(x), y^{\prime} \otimes y^{\prime \prime}\right\}=\left\{\sum(\pi q)^{-\left|x^{\prime}\right| \cdot\left|x^{\prime \prime}\right|} \pi^{p\left(x^{\prime}\right) p\left(x^{\prime \prime}\right)} x^{\prime \prime} \otimes x^{\prime}, y^{\prime} \otimes y^{\prime \prime}\right\} \\
= & \sum q^{-\left|x^{\prime}\right| \cdot\left|x^{\prime \prime}\right|} \pi^{p\left(x^{\prime}\right) p\left(x^{\prime \prime}\right)}\left\{x^{\prime \prime}, y^{\prime}\right\}\left\{x^{\prime}, y^{\prime \prime}\right\} \\
= & \sum(-1)^{\mathrm{ht}\left|x^{\prime}\right|+\mathrm{ht}\left|x^{\prime \prime}\right|} q^{\frac{-\left|x^{\prime \prime}\right| \cdot\left|y^{\prime}\right|-\left|x^{\prime}\right| \cdot\left|y^{\prime \prime}\right|-2\left|x^{\prime}\right| \cdot\left|x^{\prime \prime}\right|}{2}} q_{-\left|x^{\prime}\right|-\left|x^{\prime \prime}\right|} \\
& \quad \pi^{p\left(x^{\prime}\right) p\left(x^{\prime \prime}\right)+\frac{p\left(x^{\prime}\right) p\left(y^{\prime \prime}\right)+p\left(x^{\prime}\right)}{2}+\frac{p\left(x^{\prime \prime}\right) p\left(y^{\prime}\right)-p\left(x^{\prime \prime}\right)}{2}}\left(x^{\prime \prime}, \varrho\left(y^{\prime}\right)\right)\left(x^{\prime}, \varrho\left(y^{\prime \prime}\right)\right) \\
\stackrel{(\star)}{=} & \sum(-1)^{\mathrm{ht}|x|} q^{\frac{-|x| \cdot|y|}{2}} q_{-|x|} \pi^{\frac{p(x) p(y)+p(x)}{2}}\left(x^{\prime} \otimes x^{\prime \prime}, \varrho\left(y^{\prime \prime}\right) \otimes \varrho\left(y^{\prime}\right)\right) \\
= & (-1)^{\mathrm{ht}|x|} q^{\frac{-|x| \cdot|y|}{2}} q_{-|x|} \pi^{\frac{p(x) p(y)+p(x)}{2}}\left(x, \varrho\left(y^{\prime} y^{\prime \prime}\right)\right)
\end{aligned}
$$

where the equality $(\star)$ follows from the observation that the nonzero terms in the sum only occur when the each of the pairs $\left\{x^{\prime}, y^{\prime \prime}\right\}$ and $\left\{x^{\prime \prime}, y^{\prime}\right\}$ are of the same weight and parity. Therefore we see $y \in \mathcal{S}$. Since the algebra generators lie in $\mathcal{S}$, the claim is proved.

\subsection{Differentials and Serre relations}

Let $i \in I$. There are unique $\mathbb{Q}(q)^{\pi}$-linear maps $r_{i},{ }_{i} r:{ }^{\prime} \mathbf{f} \rightarrow$ 'f $\mathbf{f}$ such that $r_{i}(1)=$ ${ }_{i} r(1)=0$ and $r_{i}\left(\theta_{j}\right)={ }_{i} r\left(\theta_{j}\right)=\delta_{i j}$ satisfying

$$
\begin{aligned}
& { }_{i} r(x y)={ }_{i} r(x) y+\pi^{p(x) p(i)} q^{|x| \cdot i} x_{i} r(y) \\
& r_{i}(x y)=\pi^{p(y) p(i)} q^{|y| \cdot i} r_{i}(x) y+x r_{i}(y)
\end{aligned}
$$

for homogeneous $x, y \in{ }^{\prime} \mathbf{f}$. We see that if $x \in{ }^{\prime} \mathbf{f}_{\nu}$, then ${ }_{i} r(x), r_{i}(x) \in{ }^{\prime} \mathbf{f}_{\nu-i}$ and moreover that

$$
r(x)=r_{i}(x) \otimes \theta_{i}+\theta_{i} \otimes{ }_{i} r(x)+(\ldots)
$$

where (...) stands in for other bi-homogeneous terms $x^{\prime} \otimes x^{\prime \prime}$ with $\left|x^{\prime}\right| \neq i$ and $\left|x^{\prime \prime}\right| \neq i$. Therefore, we have

$$
\left(\theta_{i} y, x\right)=\left(\theta_{i}, \theta_{i}\right)\left(y,{ }_{i} r(x)\right), \quad\left(y \theta_{i}, x\right)=\left(\theta_{i}, \theta_{i}\right)\left(y, r_{i}(x)\right)
$$


for all $x, y \in{ }^{\prime} \mathbf{f}$, so ${ }_{i} r(\mathcal{I}) \cup r_{i}(\mathcal{I}) \subseteq \mathcal{I}$. Hence, both maps descend to maps on $\mathbf{f}$. It is also easy to check that

$$
r_{i} \rho=\rho_{i} r
$$

Indeed, this is trivially true for the generators, and if this holds for $x, y \in \mathbf{f}$, then

$$
\begin{aligned}
r_{i} \rho(x y) & =r_{i}(\rho(y) \rho(x))=\pi^{p(i) p(x)} q^{i \cdot|x|} r_{i}(\rho(y)) \rho(x)+\rho(y) r_{i}(\rho(x)) \\
& =\rho\left(\pi^{p(i) p(x)} q^{i \cdot|x|} x_{i} r(y)+{ }_{i} r(x) y\right)=\rho_{i} r(x y) .
\end{aligned}
$$

We define the bar-conjugate differentials $\bar{i}^{r}={ }^{-} \circ{ }_{i} r \circ{ }^{-}$and $\overline{r_{i}}={ }^{-} \circ r_{i} \circ-$. Note that they satisfy similar properties to ${ }_{i} r$ and $r_{i}$, but with $r$ and $(-,-)$ everywhere replaced with $\bar{r}$ and $\{-,-\}$. In fact, a more explicit relationship can be realized as follows.

Lemma 2.9. For any homogeneous $x \in \mathbf{f}$, we have

$$
r_{i}(x)=\pi^{p(x) p(i)-p(i) p(i)} q^{|x| \cdot i-i \cdot i} \overline{{ }_{i} r}(x) .
$$

Proof. This is trivial when $x=\theta_{i}$. Now assume this is true for $x, y \in{ }^{\prime} \mathbf{f}$. Then

$$
\begin{aligned}
& \overline{{ }_{i} r(\overline{x y})}=\overline{{ }_{i} r(\bar{x})} y+\pi^{p(x) p(i)}(\pi q)^{-|x| \cdot i} \overline{{ }_{i} r(\bar{y})} \\
& =\pi^{-p(x) p(i)+p(i) p(i)} q^{-|x| \cdot i+i \cdot i} r_{i}(x) y \\
& +\pi^{-p(y) p(i)+p(i) p(i)} q^{-|y| \cdot i+i \cdot i} \pi^{p(x) p(i)}(\pi q)^{-|x| \cdot i} x r_{i}(y) \\
& =\pi^{-p(x+y) p(i)+p(i) p(i)} q^{-|x+y| \cdot i+i \cdot i}\left(\pi^{p(y) p(i)} q^{|y| \cdot i} r_{i}(x) y+x r_{i}(y)\right) \\
& =\pi^{-p(x+y) p(i)+p(i) p(i)} q^{-|x+y| \cdot i+i \cdot i} r_{i}(x y) \text {. }
\end{aligned}
$$

The lemma is proved.

Lemma 2.10. Let $x \in \mathbf{f}_{\nu}$ where $\nu \in \mathbb{N}[I]$ is nonzero.

(a) If $r_{i}(x)=0$ for all $i \in I$, then $x=0$.

(b) If ${ }_{i} r(x)=0$ for all $i \in I$, then $x=0$. 
Proof. Suppose that $r_{i}(x)=0$ for all $i$. Using (2.20), this means that $\left(y \theta_{i}, x\right)=0$ for all $y \in \mathbf{f}$ and all $i \in I$. But since $\mathbf{f}$ is spanned by monomials in the $\theta_{i}$, this implies $x \in \mathcal{I}$, and so $x=0$ in $\mathbf{f}$. The proof of (b) proceeds similarly.

For any $n \in \mathbb{Z}$, let the divided powers $\theta_{i}^{(n)}$ (in $\mathbf{f}$ or 'f) be defined as $\theta_{i} /[n]_{q_{i}, \pi_{i}}^{!}$if $n \geq 0$ and 0 otherwise. Let ${ }_{\mathbb{A}} \mathbf{f}$ be $\mathbb{A}$-subalgebra of $\mathbf{f}$ generated by the elements $\theta_{i}^{(s)}$ for various $i \in I$ and $s \in \mathbb{Z}$. Since the generators $\theta_{i}^{(s)}$ are homogeneous, we have ${ }_{\mathbb{A}} \mathbf{f}=\bigoplus_{\nu} \mathbf{f}_{\nu}$ where $\nu$ runs over $\mathbb{N}[I]$ and ${ }_{\mathbb{A}} \mathbf{f}_{\nu}={ }_{\mathbb{A}} \mathbf{f} \cap \mathbf{f}_{\nu}$.

The next two lemmas describe the behavior of divided powers with respect to the previously defined structures on $\mathbf{f}$.

Lemma 2.11. For any $n \in \mathbb{Z}$ we have

(a) $r\left(\theta_{i}^{(n)}\right)=\sum_{t+t^{\prime}=n} q_{i}^{t t^{\prime}} \theta_{i}^{(t)} \otimes \theta_{i}^{\left(t^{\prime}\right)}$

(b) $\bar{r}\left(\theta_{i}^{(n)}\right)=\sum_{t+t^{\prime}=n}\left(\pi_{i} q_{i}\right)^{-t t^{\prime}} \theta_{i}^{(t)} \otimes \theta_{i}^{\left(t^{\prime}\right)}$.

Proof. By the quantum binomial formula (2.7) applied to $x=1 \otimes \theta_{i}$ and $y=\theta_{i} \otimes 1$, the formula follows.

Lemma 2.12. For any $n \geq 0$, we have

$$
\left(\theta_{i}^{(n)}, \theta_{i}^{(n)}\right)=\prod_{s=1}^{n} \frac{\pi_{i}^{s-1}}{1-\left(\pi_{i} q_{i}^{-2}\right)^{s}}=\pi_{i}^{n} q_{i}^{\left(\begin{array}{c}
n+1 \\
2
\end{array}\right)}\left(\pi_{i} q_{i}-q_{i}^{-1}\right)^{-n}\left([n]_{q_{i}, \pi_{i}}^{!}\right)^{-1}
$$

Proof. We prove by induction on $n$. The lemma is true by definition for $n=0,1$. For general $n$, it follows by Lemma 2.11 (a) that

$$
\begin{aligned}
\left(\theta_{i}^{(n)}, \theta_{i}^{(n)}\right) & =[n]_{q_{i}, \pi_{i}}^{-1}\left(\theta_{i}^{(n-1)} \otimes \theta_{i}, r\left(\theta_{i}^{(n)}\right)\right) \\
& =[n]_{q_{i}, \pi_{i}}^{-1}\left(\theta_{i}^{(n-1)} \otimes \theta_{i}, \sum_{t+t^{\prime}=n} q_{i}^{t t^{\prime}} \theta_{i}^{(t)} \otimes \theta_{i}^{\left(t^{\prime}\right)}\right) \\
& =[n]_{q_{i}, \pi_{i}}^{-1}\left(\theta_{i}^{(n-1)} \otimes \theta_{i}, q_{i}^{n-1} \theta_{i}^{(n-1)} \otimes \theta_{i}\right) \\
& =q_{i}^{n-1}[n]_{q_{i}, \pi_{i}}^{-1}\left(\theta_{i}, \theta_{i}\right)\left(\theta_{i}^{(n-1)}, \theta_{i}^{(n-1)}\right) .
\end{aligned}
$$


Hence by the induction hypothesis, we have

$$
\begin{aligned}
\left(\theta_{i}^{(n)}, \theta_{i}^{(n)}\right) & =q_{i}^{n-1}[n]_{q_{i}, \pi_{i}}^{-1}\left(1-\pi_{i} q_{i}^{-2}\right)^{-1} \pi_{i}^{n-1} q_{i}^{\left(\begin{array}{c}
n \\
2
\end{array}\right)}\left(\pi_{i} q_{i}-q_{i}^{-1}\right)^{-n+1}\left([n-1]_{q_{i}, \pi_{i}}^{!}\right)^{-1} \\
& =\pi_{i}^{n} q_{i}^{\left(\begin{array}{c}
n+1 \\
2
\end{array}\right)}\left(\pi_{i} q_{i}-q_{i}^{-1}\right)^{-n}\left([n]_{q_{i}, \pi_{i}}^{!}\right)^{-1} .
\end{aligned}
$$

The lemma is proved.

We are almost ready to define the essential relations in $\mathbf{f}$, but we first need the following lemma.

Lemma 2.13. Let $N \in \mathbb{N}$ and $a, a^{\prime} \in \mathbb{N}$ with $N=a+a^{\prime}$. Let $i, j, k \in I$ be pairwise distinct. Then

(a) $r_{k}\left(\theta_{i}^{(a)} \theta_{j} \theta_{i}^{\left(a^{\prime}\right)}\right)=0$,

(b) $r_{j}\left(\theta_{i}^{(a)} \theta_{j} \theta_{i}^{\left(a^{\prime}\right)}\right)=q_{i}^{a^{\prime}\langle i, j\rangle} \pi_{i}^{a^{\prime} p(j)}\left[\begin{array}{l}N \\ a^{\prime}\end{array}\right]_{q_{i}, \pi_{i}} \theta_{i}^{(N)}$,

(c) $r_{i}\left(\theta_{i}^{(a)} \theta_{j} \theta_{i}^{\left(a^{\prime}\right)}\right)=q_{i}^{a^{\prime}+(N+\langle i, j\rangle-1)} \pi_{i}^{a^{\prime}+p(j)} \theta_{i}^{(a-1)} \theta_{j} \theta_{i}^{\left(a^{\prime}\right)}+q_{i}^{a^{\prime}-1} \theta_{i}^{(a)} \theta_{j} \theta_{i}^{\left(a^{\prime}-1\right)}$.

Proof. Part (a) is clear from definitions. By (2.19) and Lemma 2.11(a) we have

$$
r_{i^{\prime}}\left(\theta_{j^{\prime}}^{(a)}\right)=\delta_{i^{\prime}, j^{\prime}} q_{i^{\prime}}^{a-1} \theta_{i^{\prime}}^{(a-1)}
$$

Parts (b) and (c) follow from this and noting

$$
r_{i}(c b a)=c b r_{i}(a)+\pi^{p(i) p(a)} q^{i \cdot|a|} c r_{i}(b) a+\pi^{p(i) p(a)+p(i) p(b)} q^{i \cdot|a|+i \cdot|b|} r_{i}(c) b a .
$$

The lemma is proved.

Proposition 2.14 (Quantum Serre relation). The generators $\theta_{i}$ of $\mathbf{f}$ satisfy the relations

$$
\sum_{n+n^{\prime}=b_{i j}}(-1)^{n^{\prime}} \pi_{i}^{n^{\prime} p(j)+\left(\begin{array}{c}
n^{\prime} \\
2
\end{array}\right)} \theta_{i}^{(n)} \theta_{j} \theta_{i}^{\left(n^{\prime}\right)}=0
$$

for any $i \neq j$ in $I$. 
Proof. By the previous lemma, we have

$$
r_{k}\left(\sum_{n+n^{\prime}=b_{i j}}(-1)^{n^{\prime}} \pi_{i}^{n^{\prime} p(j)+\left(\begin{array}{c}
n^{\prime} \\
2
\end{array}\right)} \theta_{i}^{(n)} \theta_{j} \theta_{i}^{\left(n^{\prime}\right)}\right)=0, \quad \text { for } k \neq i, j .
$$

In addition, we have

$$
\begin{aligned}
& r_{j}\left(\sum_{n+n^{\prime}=b_{i j}}(-1)^{n^{\prime}} \pi_{i}^{n^{\prime} p(j)+\left(\begin{array}{c}
n^{\prime} \\
2
\end{array}\right)} \theta_{i}^{(n)} \theta_{j} \theta_{i}^{\left(n^{\prime}\right)}\right) \\
& =\sum_{n+n^{\prime}=b_{i j}}(-1)^{n^{\prime}} \pi_{i}^{n^{\prime} p(j)+\left(\begin{array}{c}
n^{\prime} \\
2
\end{array}\right)} q_{i}^{n^{\prime}\langle i, j\rangle} \pi_{i}^{n^{\prime} p(j)}\left[\begin{array}{c}
b_{i j} \\
n^{\prime}
\end{array}\right]_{q_{i}, \pi_{i}} \theta_{i}^{\left(b_{i j}\right)} \\
& =\theta_{i}^{\left(b_{i j}\right)} \sum_{t=0}^{b_{i j}}(-1)^{t} \pi_{i}^{\left(\begin{array}{l}
t \\
2
\end{array}\right)}\left(q_{i}\right)^{t\left(1-b_{i j}\right)}\left[\begin{array}{c}
b_{i j} \\
t
\end{array}\right]_{q_{i}, \pi_{i}} .
\end{aligned}
$$

By (2.1), $1-b_{i j} \in 2 \mathbb{Z}$ if $i$ is odd, so in any case, the right-hand side of the last equation is

$$
=\theta_{i}^{\left(b_{i j}\right)} \sum_{t=0}^{b_{i j}}(-1)^{t} \pi_{i}^{\left(\begin{array}{c}
t \\
2
\end{array}\right)}\left(\pi_{i} q_{i}^{-1}\right)^{t\left(b_{i j}-1\right)}\left[\begin{array}{c}
b_{i j} \\
t
\end{array}\right]_{q_{i}, \pi_{i}}=0
$$

where the last equality follows from (2.13). Finally,

$$
\begin{aligned}
& r_{i}\left(\sum_{n+n^{\prime}=b_{i j}}(-1)^{n^{\prime}} \pi_{i}^{n^{\prime} p(j)+\left(\begin{array}{c}
n^{\prime} \\
2
\end{array}\right)} \theta_{i}^{(n)} \theta_{j} \theta_{i}^{\left(n^{\prime}\right)}\right) \\
& =\sum_{n+n^{\prime}=b_{i j}}(-1)^{n^{\prime}} \pi_{i}^{n^{\prime} p(j)+\left(\begin{array}{c}
n^{\prime} \\
2
\end{array}\right)} q_{i}^{n^{\prime}} \pi_{i}^{n^{\prime}+p(j)} \theta_{i}^{(n-1)} \theta_{j} \theta_{i}^{\left(n^{\prime}\right)} \\
& \quad+\sum_{n+n^{\prime}=b_{i j}}(-1)^{n^{\prime}} \pi_{i}^{n^{\prime} p(j)+\left(\begin{array}{c}
n^{\prime} \\
2
\end{array}\right)} q_{i}^{n^{\prime}-1} \theta_{i}^{(n)} \theta_{j} \theta_{i}^{\left(n^{\prime}-1\right)} \\
& =\sum_{t=0}^{b_{i j}-1}(-1)^{t} \pi_{i}^{t p(j)+p(j)+\left(\begin{array}{c}
t+1 \\
2
\end{array}\right)} q_{i}^{t} \theta_{i}^{\left(b_{i j}-1-t\right)} \theta_{j} \theta_{i}^{(t)} \\
& \quad-\sum_{t=0}^{b_{i j}-1}(-1)^{t} \pi_{i}^{(t+1) p(j)+\left(\begin{array}{c}
t+1 \\
2
\end{array}\right)} q_{i}^{t} \theta_{i}^{\left(b_{i j}-1-t\right)} \theta_{j} \theta_{i}^{(t)} \\
& =0 .
\end{aligned}
$$

Now Proposition 2.14 follows by Lemma 2.10. 
Remark 2.15. Note that the bar map ${ }^{-}$on $\mathbf{f}$ may not be well-defined if we do not assume that the Cartan datum is bar-consistent. For example, suppose $(I, \cdot)$ has $i, j \in I_{\overline{0}}$ with $i \cdot j=-1$, hence $d_{i}=d_{j}=1$. Then the calculations above hold; that $i s, S_{i j}:=\theta_{i}^{(2)} \theta_{j}-\theta_{i} \theta_{j} \theta_{i}+\theta_{j} \theta_{i}^{(2)}=0$; however, since $\overline{[2]_{i}}=\pi[2]_{i}$, it is easy to see that $\overline{S_{i j}} \notin \mathcal{I}$.

In fact, the quantum Serre relations generate all other relations.

Proposition 2.16. The ideal $\mathcal{I}$ of 'f is generated by the Serre relations.

Remark 2.17. An alternate proof of this result using a $\mathbf{U}$-module character formula argument is given in [CHW1] using the results in [BKM]. We will present an independent proof in the next section, which instead uses some general results about "twisted bialgebras"; see the appendix for details of these structures.

\subsection{The twistor isomorphism}

Let $\mathbf{t}$ be a square root of -1 . For a $\operatorname{ring} R$ and $R$-module $M$ (resp. $R$-algebra $A$ ), let $M[\mathbf{t}]=R[\mathbf{t}] \otimes_{R} M$ (resp. $A[\mathbf{t}]=R[\mathbf{t}] \otimes_{R} A$ ) be the corresponding scalar extension. In particular, we consider the $\mathbb{Q}(q, \mathbf{t})^{\pi}$-algebra $\mathbf{f}[\mathbf{t}]$. We define $\mathbf{t}_{i}=\mathbf{t}^{d_{i}}$.

Definition 2.18. An enhancer $\phi$ is an function $\phi: \mathbb{Z}[I] \times X \rightarrow \mathbb{Z} / 4 \mathbb{Z}$ satisfying

(a) $\phi\left(\nu, \lambda+\mu^{\prime}\right)=\phi\left(\nu, \mu^{\prime}\right)+\phi(\nu, \lambda)$ and $\phi(\nu+\mu, \lambda)=\phi(\nu, \lambda)+\phi(\mu, \lambda)$ for $\nu, \mu \in \mathbb{N}[I]$ and $\lambda \in X$.

(b) $\phi\left(i, j^{\prime}\right) \in 2 \mathbb{Z} / 4 \mathbb{Z}$ for $i \neq j \in I$.

(c) $\phi\left(i, j^{\prime}\right)-\phi\left(j, i^{\prime}\right)=i \cdot j+2 p(i) p(j)$ and $\phi\left(i, i^{\prime}\right)=d_{i}$ for $i \neq j \in I$.

Remark 2.19. A particular choice of enhancer is defined in [CFLW] where the Cartan datum is $X$-regular, so such an enhancer certainly exists. The regularity condition is not necessary if we take the restriction $\left.\phi\right|_{\mathbb{Z}[I] \times \mathbb{Z}[I]}$, hence for many results pertaining 
to $\mathbf{f}$ we need not assume $X$-regularity. Nonetheless, we will brush this matter under the carpet and henceforth we will always assume an enhancer exists.

Fix a choice of $\phi$. Define a new multiplication $*$ on $\mathbf{f}[\mathbf{t}]$ by setting

$$
x * y=t^{\phi\left(|x|,|y|^{\prime}\right)} x y
$$

Since $\phi$ is bilinear and the original multiplication was associative, $(\mathbf{f}[\mathbf{t}], *)$ is a $\mathbb{N}[I]$ graded associative algebra generated by $\theta_{i}$. We will use the notation

$$
x^{* n}=\underbrace{x * x * \ldots * x}_{n}
$$

for powers taken with respect $*$. The eponymous isomorphism of the section is described in the following lemma.

Lemma 2.20. There is a $\mathbb{Q}(\mathbf{t})$-algebra isomorphism $\mathfrak{X}: \mathbf{f}[\mathbf{t}] \rightarrow \mathbf{f}[\mathbf{t}]$, called a twistor, defined by

$$
\mathfrak{X}\left(\theta_{i}\right)=\theta_{i}(i \in I), \quad \mathfrak{X}(q)=-\mathbf{t} q, \quad \mathfrak{X}(\pi)=-\pi, \quad \mathfrak{X}(x y)=\mathfrak{X}(x) * \mathfrak{X}(y) .
$$

Proof. Set

$$
S_{i j}=\sum_{k=0}^{b_{i j}}(-1)^{k}(-\pi)^{\left(\begin{array}{c}
k \\
2
\end{array}\right) p(i)+k p(i) p(j)}\left[\begin{array}{c}
b_{i j} \\
k
\end{array}\right]_{\mathbf{t}_{i}^{-1} q_{i},(-\pi)_{i}} \theta_{i}^{* b_{i j}-k} * \theta_{j} * \theta_{i}^{* k} .
$$

To show such a $\mathbb{Q}(\mathbf{t})$-linear map $\mathfrak{X}$ exists, it suffices to show that the images of the generators satisfy $(2.21)$ with respect to $*$; that is,

$$
S_{i j}=0 \quad \text { for all } i \neq j \in I
$$

To that end, let $i, j \in I$ such that $i \neq j$. Unraveling the definition of $*$, we have

$$
\begin{aligned}
& S_{i j}=\sum_{k=0}^{b_{i j}}(-1)^{k}(-\pi)^{\left(\begin{array}{l}
k \\
2
\end{array}\right) p(i)+k p(i) p(j)}\left[\begin{array}{c}
b_{i j} \\
k
\end{array}\right]_{\mathbf{t}_{i}^{-1} q_{i},(-\pi)_{i}} \\
& \times \mathbf{t}^{\left(\left(\begin{array}{c}
k \\
2
\end{array}\right)+\left(\begin{array}{c}
b_{i j}-k \\
2
\end{array}\right)+k\left(b_{i j}-k\right)\right) d_{i}+\left(b_{i j}-k\right) \phi\left(i, j^{\prime}\right)+k \phi\left(j, i^{\prime}\right)} \theta_{i}^{b_{i j}-k} \theta_{j} \theta_{i}^{k} .
\end{aligned}
$$


One verifies that $\left(\begin{array}{c}k \\ 2\end{array}\right)+\left(\begin{array}{c}b_{i j}-k \\ 2\end{array}\right)=\left(\begin{array}{c}b_{i j} \\ 2\end{array}\right)-k\left(b_{i j}-k\right)$ and

$$
\left[\begin{array}{c}
b_{i j} \\
k
\end{array}\right]_{\mathbf{t}_{i}^{-1} q_{i},(-\pi)_{i}}=\mathbf{t}^{k\left(b_{i j}-k\right) d_{i}}\left[\begin{array}{c}
b_{i j} \\
k
\end{array}\right]_{q_{i}, \pi_{i}} .
$$

Using these identities, we rewrite $S_{i j}$ as

$$
\begin{aligned}
\mathbf{t}^{-\left(\begin{array}{c}
b_{i j} \\
2
\end{array}\right) d_{i}} S_{i j} & =\sum_{k=0}^{b_{i j}}(-1)^{k}(-\pi)^{\left(\begin{array}{c}
k \\
2
\end{array}\right) p(i)+k p(i) p(j)}\left[\begin{array}{c}
b_{i j} \\
k
\end{array}\right]_{\mathbf{t}_{i}^{-1} q_{i},(-\pi)_{i}} \mathbf{t}^{\left(b_{i j}-k\right) \phi\left(i, j^{\prime}\right)+k \phi(j, i)} \theta_{i}^{b_{i j}-k} \theta_{j} \theta_{i}^{k} \\
& =\sum_{k=0}^{b_{i j}}(-1)^{k}(-\pi)^{\left(\begin{array}{c}
k \\
2
\end{array}\right) p(i)+k p(i) p(j)}\left[\begin{array}{c}
b_{i j} \\
k
\end{array}\right]_{q_{i}, \pi_{i}} \mathbf{t}^{\mathbf{2}} \theta_{i}^{b_{i j}-k} \theta_{j} \theta_{i}^{k}
\end{aligned}
$$

where

$$
\begin{aligned}
\boldsymbol{\beta} & =k\left(b_{i j}-k\right) d_{i}+\left(b_{i j}-k\right) \phi\left(i, j^{\prime}\right)+k \phi\left(j, i^{\prime}\right) \\
& =k\left(1-a_{i j}-k\right) d_{i}+b_{i, j} \phi\left(i, j^{\prime}\right)+k(i \cdot j+2 p(i) p(j)) \\
& =i \cdot j+b_{i, j} \phi\left(i, j^{\prime}\right)+2\left(\left(\begin{array}{c}
k \\
2
\end{array}\right) p(i)+k p(i) p(j)\right) \quad(\bmod 4) .
\end{aligned}
$$

Then we can rewrite (2.25) and apply the Serre relation (2.21) for $\mathbf{f}$ to conclude that

$$
\mathbf{t}^{-\left(\begin{array}{c}
b_{i j} \\
2
\end{array}\right) d_{i}-i \cdot j-b_{i, j} \phi\left(i, j^{\prime}\right)} S_{i j}=\sum_{k=0}^{b_{i j}}(-1)^{k} \pi^{\left(\begin{array}{c}
k \\
2
\end{array}\right) p(i)+k p(i) p(j)}\left[\begin{array}{c}
b_{i j} \\
k
\end{array}\right]_{q_{i}, \pi_{i}} \theta_{i}^{b_{i j}-k} \theta_{j} \theta_{i}^{k}=0 .
$$

Therefore, (2.24) is verified and $\Psi$ is well defined.

Finally, to see that $\mathfrak{X}$ is an isomorphism we can observe that since products in $\mathbf{f}[\mathbf{t}]$ and $(\mathbf{f}[\mathbf{t}], *)$ are equal up to a scalar, the Serre presentation for $\mathbf{f}$ induces a presentation for $(\mathbf{f}, *)$ by rewriting the Serre relations in terms of $*$. In particular, a similar argument can be used to show that a map $\mathfrak{Y}:(\mathbf{f}[\mathbf{t}], *) \rightarrow \mathbf{f}[\mathbf{t}]$ satisfying

$$
\mathfrak{Y}\left(\theta_{i}\right)=\theta_{i}, \quad \mathfrak{Y}(q)=\mathbf{t} q, \quad \mathfrak{Y}(\pi)=-\pi, \quad \mathfrak{Y}(x * y)=\mathfrak{Y}(x) \mathfrak{Y}(y),
$$

is well defined as well; clearly $\mathfrak{Y}$ is the inverse of $\mathfrak{X}$.

A particularly useful property of the twistor isomorphism is that it restricts to a $\mathbb{Q}(\mathbf{t})$-vector space isomorphism of $\left.\mathbf{f}[\mathbf{t}]\right|_{\pi=1}$ and $\left.\mathbf{f}[\mathbf{t}]\right|_{\pi=-1}$, thus allowing us to compare the non-super and super aspects of $\mathbf{f}$. 
Remark 2.21. It follows from the map $\mathfrak{X}$ that the canonical basis of $\left.\mathbf{f}\right|_{\pi=1}$ induces a $\mathbb{Q}\left(\mathbf{t}^{-1} q\right)$-basis of $\left.\mathbf{f}[\mathbf{t}]\right|_{\pi=-1}$. Moreover, we shall see that $\mathfrak{X}$ commutes with the respective bar involutions, so this basis is bar-invariant. However, it is

It turns out that this isomorphism is forced to exist by some general structure theory, and this perspective allows us to prove that the Serre relations generate all the relations.

\section{Sketch of proof of Proposition 2.16}

First, the algebra ' $\mathbf{f}[\mathbf{t}]$ is an example of a twisted bialgebra over $\mathbb{Q}(q, \mathbf{t})^{\pi}$; see the appendix for a discussion of the structure of such algebras. The twistor $\mathfrak{X}$ defined above is just an explicit description of an isomorphism of nondegenerate twisted bialgebras. Indeed, $(\mathbf{f}[\mathbf{t}], *)$ is just the $t$-twist of $\mathbf{f}[\mathbf{t}]$, where $t(\mu, \nu)=\mathbf{t}^{\phi\left(\mu, \nu^{\prime}\right)}$. In particular, one can show it is the unique (up to isomorphism) nondegenerate twisted bialgebra of type $(I, \diamond, 1)$ for a particular bicharacter $\diamond$. On the other hand, $\mathbf{f}[\mathbf{t}]$ is a nondegenerate twisted bialgebra of type $(I, \bullet, 1)$ up to the change of parameters $q \mapsto \mathbf{t}^{-1} q$ and $\pi \mapsto-\pi$, so it must be isomorphic to (f $\left.[\mathbf{t}], *\right)$. In particular, this provides an alternate proof of Lemma 2.20 which is independent of Proposition 2.16.

Now, the twistor lifts easily to an isomorphism of 'f $\mathbf{f}[\mathbf{t}]$ and ('f $[\mathbf{t}], *)$. By the computations in the proof of Lemma 2.20, the image of a Serre relation under this isomorphism is proportional to itself. On the other hand, $\left.\mathcal{I}\right|_{\pi=1}$ is known to be generated by the Serre relations. Then their images generate $\left.\mathcal{I}[\mathbf{t}]\right|_{\pi=-1}$, hence the Serre relations themselves are generators and lie in $\left.\mathcal{I}\right|_{\pi=-1}$ finishing the proof.

It will be useful to see how the map $\mathfrak{X}$ intertwines with the maps ${ }^{-}, \varrho, r_{i}$, and ${ }_{i} r$ defined on $\mathbf{f}$ above.

Proposition 2.22. For all $x \in \mathbf{f}$, we have $\mathfrak{X}(\bar{x})=\overline{\mathfrak{X}(x)}$.

Proof. Since both maps are $\mathbb{Q}(\mathbf{t})$-algebra isomorphisms, it suffices to show the equalities on the generators. First note that the statement is clear for $x=\theta_{i}$ for $i \in I$ and 
$x=\pi$. It remains to verify this for $x=q$ :

$$
\mathfrak{X}(\bar{q})=\mathfrak{X}\left(\pi q^{-1}\right)=-\mathbf{t} \pi q^{-1}=\overline{\mathbf{t}^{-1} q}=\overline{\mathfrak{X}(q)} .
$$

For $i_{1}, \ldots, i_{n} \in I$, we denote

$$
\begin{aligned}
& \mathbf{N}\left(i_{1}+\ldots+i_{n}\right)=\sum_{1 \leq r<s \leq n} i_{r} \cdot i_{s}, \\
& \mathbf{p}\left(i_{1}+\ldots+i_{n}\right)=\sum_{1 \leq r<s \leq n} p\left(i_{r}\right) p\left(i_{s}\right) .
\end{aligned}
$$

By convention, $\mathbf{N}\left(i_{1}\right)=\mathbf{p}\left(i_{1}\right)=0$. Note that $\mathbf{N}(\cdot)$ is always an even integer by (2.1).

Proposition 2.23. The involutions $\mathfrak{X} \varrho \mathfrak{X}^{-1}$ and $\varrho$ on $\mathbf{f}[\mathbf{t}]$ are equal up to a sign on each weight space. More precisely, we have

$$
\Psi \varrho \Psi^{-1}(x)=(-1)^{\frac{\mathbf{N}(\nu)}{2}+\mathbf{p}(\nu)} \varrho(x), \quad \text { for } x \in \mathbf{f}[\mathbf{t}]_{\nu} .
$$

Proof. We prove the formula (2.26) by induction on the height ht $(|x|)$. The formula clearly holds when $h t(|x|) \leq 1$.

Now assume that the formula holds for $x$ with ht $(|x|) \geq 1$ and for $y$ with ht $(|y|) \geq$ 1. Recall $\mathbf{t}^{2}=-1$. Then applying the definitions, we have

$$
\begin{aligned}
\Psi \varrho \Psi^{-1}(x * y) & =\Psi\left(\varrho\left(\Psi^{-1}(y)\right) \varrho\left(\Psi^{-1}(x)\right)\right) \\
& =\Psi \varrho \Psi^{-1}(y) * \Psi \varrho \Psi^{-1}(x) \\
& =(-1)^{\frac{\mathrm{N}(|y|)}{2}+\mathbf{p}(|y|)+\frac{\mathrm{N}(|x|)}{2}+\mathbf{p}(|x|)} \varrho(y) * \varrho(x) \\
& =(-1)^{\frac{\mathrm{N}(|y|)}{2}+\mathbf{p}(|y|)+\frac{\mathrm{N}(|x|)}{2}+\mathbf{p}(|x|)} \mathbf{t}^{\phi(|y|,|x|)-\phi(|x|,|y|)} \varrho(x * y) \\
& =(-1)^{\frac{\mathrm{N}(|x * y|)}{2}+\mathbf{p}(|x * y|)} \varrho(x * y) .
\end{aligned}
$$

Hence the formula (2.26) holds for $x * y$. This completes the induction.

Since $\mathbf{N}$ and $\mathbf{p}$ only depend on the weight, $\Psi \varrho \Psi^{-1}$ and $\varrho$ are proportional on each weight space. The proposition is proved. 
Lemma 2.24. For any $x \in \mathbf{f}[\mathbf{t}]_{\nu}$,

$$
\mathfrak{X}\left({ }_{i} r(x)\right)=\mathbf{t}^{-\phi\left(i, \nu^{\prime}-i^{\prime}\right)}{ }_{i} r(\mathfrak{X}(x)), \quad \mathfrak{X}\left(r_{i}(x)\right)=\mathbf{t}^{-\phi\left(\nu-i, i^{\prime}\right)} r_{i}(\mathfrak{X}(x)) .
$$

Proof. This is a straightforward verification. 


\section{Chapter 3}

\section{The quantum covering group $\mathrm{U}$}

\subsection{The algebras ' $U$ and $U$}

Definition 3.1. [CHW1] The quantum covering group $\mathbf{U}$ associated to the root datum $((I, \cdot), Y, X,\langle-,-\rangle)$ is the $\mathbb{Q}(q)^{\pi}$-algebra with generators $E_{i}, F_{i}, K_{\mu}$, and $J_{\mu}$, for $i \in I$ and $\mu \in Y$, subject to the relations:

$$
\begin{gathered}
J_{\mu} J_{\nu}=J_{\mu+\nu}, \quad K_{\mu} K_{\nu}=K_{\mu+\nu}, \quad K_{0}=J_{0}=J_{\nu}^{2}=1, \quad J_{\mu} K_{\nu}=K_{\nu} J_{\mu} \\
J_{\mu} E_{i}=\pi^{\left\langle\mu, i^{\prime}\right\rangle} E_{i} J_{\mu}, \quad J_{\mu} F_{i}=\pi^{-\left\langle\mu, i^{\prime}\right\rangle} F_{i} J_{\mu}, \\
K_{\mu} E_{i}=q^{\left\langle\mu, i^{\prime}\right\rangle} E_{i} K_{\mu}, \quad K_{\mu} F_{i}=q^{-\left\langle\mu, i^{\prime}\right\rangle} F_{i} K_{\mu}, \\
E_{i} F_{j}-\pi^{p(i) p(j)} F_{j} E_{i}=\delta_{i j} \frac{J_{d_{i} i} K_{d_{i} i}-K_{-d_{i} i}}{\pi_{i} q_{i}-q_{i}^{-1}} \\
\sum_{k=0}^{b_{i j}}(-1)^{k} \pi^{\left(\begin{array}{l}
k \\
2
\end{array}\right) p(i)+k p(i) p(j)}\left[\begin{array}{c}
b_{i j} \\
k
\end{array}\right]_{q_{i}, \pi_{i}} E_{i}^{b_{i j}-k} E_{j} E_{i}^{k}=0 \quad(i \neq j), \\
\sum_{k=0}^{b_{i j}}(-1)^{k} \pi^{\left(\begin{array}{c}
k \\
2
\end{array}\right) p(i)+k p(i) p(j)}\left[\begin{array}{c}
b_{i j} \\
k
\end{array}\right]_{q_{i}, \pi_{i}} F_{i}^{b_{i j}-k} F_{j} F_{i}^{k}=0 \quad(i \neq j),
\end{gathered}
$$

for $i, j \in I$ and $\mu, \nu \in Y$.

We also consider the associative $\mathbb{Q}(q)^{\pi}$-algebra ' $\mathbf{U}$ (with 1 ) defined by the generators

$$
E_{i} \quad(i \in I), \quad F_{i} \quad(i \in I), \quad J_{\mu} \quad(\mu \in Y), \quad K_{\mu} \quad(\mu \in Y)
$$

only the relations (3.1)-(3.4) above. 
From Proposition 2.16, we see that there are well-defined algebra homomorphisms $\mathbf{f} \rightarrow \mathbf{U}, x \mapsto x^{+}$(with image denoted by $\mathbf{U}^{+}$) and $\mathbf{f} \rightarrow \mathbf{U}, x \mapsto x^{-}$(with image denoted by $\mathbf{U}^{-}$) such that $E_{i}=\theta_{i}^{+}$and $F_{i}=\theta_{i}^{-}$for all $i \in I$. Clearly, there are well defined algebra homomorphisms 'f $\rightarrow{ }^{\prime} \mathbf{U}$ with the aforementioned properties.

As a matter of convenience, we will use the notations

$$
\tilde{J}_{\nu}=J_{\tilde{\nu}}, \quad \tilde{K}_{\nu}=K_{\tilde{\nu}}
$$

for $\nu \in \mathbb{Z}[I]$, where $\tilde{\nu}$ is defined as in $(2.3)$.

(In terms of standard notations used in some other quantum group literature, it is understood that $K_{\mu}=q^{\mu}$ and $\tilde{K}_{i}=q^{h_{i}}$. It is instructive to see our new generators $J^{\prime}$ 's can be understood in the same vein as $J_{\mu}=\pi^{\mu}$ and $\tilde{J}_{i}=\pi^{h_{i}}$.)

For any $p \geq 0$, we set $E_{i}^{(p)}=\left(\theta_{i}^{(p)}\right)^{+}$and $F_{i}^{(p)}=\left(\theta_{i}^{(p)}\right)^{-}$.

Example 3.2. In the case $I=I_{\overline{1}}=\{I\}$, we can identify $Y=X=\mathbb{Z}$ with $i=1 \in Y$, $i^{\prime}=2 \in X$, and $\langle\mu, \lambda\rangle=\mu \lambda$. Then $\mathbf{U}$ is the $\mathbb{Q}(q)^{\pi}$-algebra generated by $E, F, K, J$ such that

$$
\begin{gathered}
J K=K J, \quad J E=E J, \quad J F=F J, \quad J^{2}=1, \\
K E K=q^{2} E, \quad K F K=q^{-2} F, \\
E F-\pi F E=\frac{J K-K^{-1}}{\pi q-q^{-1}} .
\end{gathered}
$$

Note that the quotient algebras $\mathbf{U} /((J \pm 1) \mathbf{U})$ are isomorphic to the two variants of the quantum group $\mathbf{U}_{q}(\mathfrak{o s p}(1 \mid 2))$ defined in $[C l W]$.

By inspection, there is a unique algebra automorphism (of order 4 ) $\omega:{ }^{\prime} \mathbf{U} \rightarrow{ }^{\prime} \mathbf{U}$ such that

$$
\omega\left(E_{i}\right)=F_{i}, \quad \omega\left(F_{i}\right)=\pi_{i} \tilde{J}_{i} E_{i}, \quad \omega\left(K_{\mu}\right)=K_{-\mu}, \quad \omega\left(J_{\mu}\right)=J_{\mu}
$$

for $i \in I, \mu \in Y$. We have $\omega\left(x^{+}\right)=x^{-}$and $\omega\left(x^{-}\right)=\pi_{|x|} \tilde{J}_{|x|} x^{+}$for all $x \in \mathbf{f}$, and thus the same formula defines a unique algebra automorphism $\omega: \mathbf{U} \rightarrow \mathbf{U}$. 
Similarly, there is a unique isomorphism of $\mathbb{Q}(q)^{\pi}$-vector spaces $\varrho:{ }^{\prime} \mathbf{U} \rightarrow{ }^{\prime} \mathbf{U}$ such that

$$
\varrho\left(E_{i}\right)=E_{i}, \quad \varrho\left(F_{i}\right)=\pi_{i} \tilde{J}_{i} F_{i}, \quad \varrho\left(K_{\mu}\right)=K_{-\mu}, \quad \varrho\left(J_{\mu}\right)=J_{\mu}
$$

for $i \in I, \mu \in Y$ such that $\varrho\left(u u^{\prime}\right)=\varrho\left(u^{\prime}\right) \varrho(u)$ for $u, u^{\prime} \in \mathbf{U}$. We have

$$
\varrho\left(x^{+}\right)=\varrho(x)^{+}, \quad \varrho\left(x^{-}\right)=\pi_{|x|} \tilde{J}_{|x|} \varrho(x)^{-}, \quad \forall x \in \mathbf{f} .
$$

Again, this implies that the same formula defines a unique algebra automorphism $\varrho: \mathbf{U} \rightarrow \mathbf{U}$. Note that $\varrho$ on $\mathbf{U}^{+}$matches exactly $\varrho$ on $\mathbf{f}$, but $\varrho$ on $\mathbf{U}^{-}$looks quite different from $\varrho$ on $\mathbf{f}$ (in contrast to the quantum group setting [Lu4]).

Finally, the bar-involution on $\mathbf{f}$ extends to an automorphism of $\mathbf{U}$. Specifically, there is a $\mathbb{Q}^{\pi}$-algebra automorphism ${ }^{-}$defined by

$$
\bar{E}_{i}=E_{i}, \quad \bar{F}_{i}=F_{i}, \quad \bar{K}_{\nu}=J_{\nu} K_{-\nu}, \quad \bar{J}_{\nu}=J_{\nu}, \quad \bar{q}=\pi q^{-1} .
$$

We also have a $\mathbb{Q}^{\pi}$-linear automorphism $\dagger$ on $\mathbf{U}$ extending $\dagger: \mathbb{Q}(q)^{\pi} \rightarrow \mathbb{Q}(q)^{\pi}$.

Lemma 3.3. There exists a $\mathbb{Q}^{\pi}$-algebra automorphism $\dagger: \mathbf{U} \rightarrow \mathbf{U}$, denoted by $\cdot \mapsto \cdot \cdot^{\dagger}$, such that

$$
E_{i}^{\dagger}=\pi_{i} \tilde{J}_{i} E_{i}, \quad F_{i}^{\dagger}=F_{i}, \quad K_{\nu}^{\dagger}=J_{\nu} K_{\nu}, \quad J_{\nu}^{\dagger}=J_{\nu}, \quad q^{\dagger}=\pi q .
$$

Proof. To see that $\dagger$ is a well-defined map, we may check that the images of the generators satisfy the defining relations. All of the relations are trivial to verify except the Serre relations when $p(i) \neq 0$. However, since $b_{i j}=1-a_{i j}$ is odd in this case, the binomial coefficients are dagger-invariant. Then (3.6) is dagger-invariant, and the image of (3.5) is proportional to itself (by a factor of $\pi^{b_{i j} p(i)+p(j)} \tilde{J}_{b_{i j} i+j}$ ).

Remark 3.4. One interpretation of the automorphism $\dagger$ is as follows. There is an algebra $\mathbf{U}^{\dagger}$ with generators $E_{i}^{\dagger}, F_{i}^{\dagger}, J_{\nu}^{\dagger}, K_{\nu}^{\dagger}(i \in I, \nu \in Y)$, and subject to (3.1)-(3.6) (with $E_{i}, F_{i}, J_{\nu}, K_{\nu}$ replaced by $E_{i}^{\dagger}, F_{i}^{\dagger}, J_{\nu}^{\dagger}, K_{\nu}^{\dagger}$ ) except with (3.4) replaced by

$$
E_{i}^{\dagger} F_{j}^{\dagger}-\pi^{p(i) p(j)} F_{j}^{\dagger} E_{i}^{\dagger}=\delta_{i j} \frac{\tilde{K}_{i}^{\dagger}-\tilde{J}_{i}^{\dagger} \tilde{K}_{-i}^{\dagger}}{q_{i}-\pi_{i} q_{i}^{-1}}
$$


The algebra $\mathbf{U}^{\dagger}$ may be thought of as $\mathbf{U}$ defined with respect to the alternate convention of $(v, \pi)$-integers, and $\dagger$ defines a $\mathbb{Q}^{\pi}$-algebra isomorphism $\dagger: \mathbf{U} \rightarrow \mathbf{U}^{\dagger}$ defined by

$$
E_{i} \mapsto E_{i}^{\dagger}, \quad F_{i} \mapsto F_{i}^{\dagger}, \quad K_{\nu} \mapsto J_{\nu}^{\dagger} K_{\nu}^{\dagger}, \quad J_{\nu} \mapsto J_{\nu}^{\dagger}, \quad v \mapsto \pi v
$$

For $\mathbb{Z}_{2}$-homogeneous elements $x, y \in \mathbf{U}$, we write the supercommutator as $[x, y]:=$ $x y-\pi^{p(x) p(y)} y x$. The relation (3.4) can be generalized to the following commutator identities.

Proposition 3.5. For $x \in{ }^{\prime} \mathbf{f}$ and $i \in I$, we have (in ' $\mathbf{U}$ )

(a) $\left[x^{+}, F_{i}\right]=\frac{r_{i}(x)^{+} \tilde{J}_{i} \tilde{K}_{i}-\tilde{K}_{-i} \pi_{i}^{p(x)-p(i)}{ }_{i} r(x)^{+}}{\pi_{i} q_{i}-q_{i}^{-1}}$,

(b) $\left[E_{i}, x^{-}\right]=\frac{\tilde{J}_{i} \tilde{K}_{i}{ }_{i} r(x)^{-}-\pi_{i}^{p(x)-p(i)} r_{i}(x)^{-} \tilde{K}_{-i}}{\pi_{i} q_{i}-q_{i}^{-1}}$.

Proof. Assume that (a) is known for $x^{\prime}$ and $x^{\prime \prime}$; we shall show it holds for $x=x^{\prime} x^{\prime \prime}$. Let $y^{\prime}=\left(x^{\prime}\right)^{+},{ }_{i} y^{\prime}={ }_{i} r\left(x^{\prime}\right)^{+}$and similarly for $r_{i}, x^{\prime \prime}$ and $x$.

$$
\begin{aligned}
y F_{i}= & \pi_{i}^{p\left(x^{\prime \prime}\right)} y^{\prime} F_{i} y^{\prime \prime}+\frac{y^{\prime} y_{i}^{\prime \prime} \tilde{J}_{i} \tilde{K}_{i}-y^{\prime} \tilde{K}_{-i} \pi_{i}^{p\left(x^{\prime \prime}\right)-p(i)}{ }_{i} y^{\prime \prime}}{\pi_{i} q_{i}-q_{i}^{-1}} \\
= & \pi_{i}^{p\left(x^{\prime} x^{\prime \prime}\right)} F_{i} y+\pi_{i}^{p\left(x^{\prime \prime}\right)} \frac{y_{i}^{\prime} \tilde{J}_{i} \tilde{K}_{i} y^{\prime \prime}-\tilde{K}_{-i} \pi_{i}^{p\left(x^{\prime}\right)-p(i)}{ }_{i} y^{\prime} y^{\prime \prime}}{\pi_{i} q_{i}-q_{i}^{-1}} \\
& +\frac{y^{\prime} y_{i}^{\prime \prime} \tilde{J}_{i} \tilde{K}_{i}-y^{\prime} \tilde{K}_{-i} \pi_{i}^{p\left(x^{\prime \prime}\right)-p(i)}{ }_{i} y^{\prime \prime}}{\pi_{i} q_{i}-q_{i}^{-1}} \\
= & \pi^{p\left(x^{\prime} x^{\prime \prime}\right) p(i)} F_{i} y+\frac{y_{i} \tilde{J}_{i} \tilde{K}_{i}-\tilde{K}_{-i} \pi_{i}^{p(x)-p(i)}{ }_{i} y}{\pi_{i} q_{i}-q_{i}^{-1}}
\end{aligned}
$$

Since (a) holds for the generators, it holds for all $x \in{ }^{\prime} \mathbf{f}$.

If we apply $\omega^{-1}$, we obtain

$$
\pi_{i} \tilde{J}_{i} x^{-} E_{i}-\pi_{i}^{p(x)-p(i)} \tilde{J}_{i} E_{i} x^{-}=\frac{r_{i}(x)^{-} \tilde{J}_{i} \tilde{K}_{-i}-\tilde{K}_{i} \pi_{i}^{p(x)-p(i)}{ }_{i} r(x)^{-}}{\pi_{i} q_{i}-q_{i}^{-1}},
$$

and multiplying both sides by $\pi_{i}^{p(x)-p(i)} \tilde{J}_{i}$ establishes (b). 
The following corollary follows by applying the bar involution to the above identities.

Corollary 3.6. For $x \in{ }^{\prime} \mathbf{f}$ and $i \in I$, we have (in 'U )

$\begin{aligned} \text { (a) }\left[x^{+}, F_{i}\right] & =\frac{\pi_{i}^{p(x)-p(i)} \tilde{J}_{i} \tilde{K}_{i} \bar{i} r(x)^{+}-\bar{r}_{i}(x)^{+} \tilde{K}_{-i}}{\pi_{i} q_{i}-q_{i}^{-1}}, \\ \text { (b) }\left[E_{i}, x^{-}\right] & =\frac{\pi_{i}^{p(x)-p(i)} \bar{r}_{i}(x)^{-} \tilde{J}_{i} \tilde{K}_{i}-\tilde{K}_{-i} \bar{i}^{r}(x)^{-}}{\pi_{i} q_{i}-q_{i}^{-1}} .\end{aligned}$

We record the following formulas for further use.

Lemma 3.7. For any $N, M \geq 0$ we have in $\mathbf{U}$ or ${ }^{\prime} \mathbf{U}$

$$
\begin{aligned}
& E_{i}^{(N)} F_{i}^{(M)}=\sum_{t} \pi_{i}^{M N-\left(\begin{array}{c}
t+1 \\
2
\end{array}\right)} F_{i}^{(M-t)}\left[\begin{array}{c}
i ; 2 t-M-N \\
t
\end{array}\right] E_{i}^{(N-t)}, \\
& F_{i}^{(N)} E_{i}^{(M)}=\sum_{t}(-1)^{t} \pi_{i}^{(M-t)(N-t)-t^{2}} E_{i}^{(M-t)}\left[\begin{array}{c}
M+N-(t+1) \\
t
\end{array}\right] F_{i}^{(N-t)}, \\
& E_{i}^{(N)} F_{j}^{(M)}=\pi^{M N p(i) p(j)} F_{j}^{(M)} E_{i}^{(N)} \quad \text { if } i \neq j,
\end{aligned}
$$

where

$$
\left[\begin{array}{c}
\nu ; a \\
t
\end{array}\right]=\prod_{s=1}^{t} \frac{\left(\pi_{\nu} q_{\nu}\right)^{a-s+1} \tilde{J}_{\nu} \tilde{K}_{\nu}-q_{\nu}^{s-a-1} \tilde{K}_{-\nu}}{\left(\pi_{\nu} q_{\nu}\right)^{s}-q_{\nu}^{-s}}
$$

Let ${ }_{\mathbb{A}} \mathbf{U}^{ \pm}={ }_{\mathbb{A}} \mathbf{f}^{ \pm}$. We define ${ }_{\mathbb{A}} \mathbf{U}$ to be the $\mathbb{A}$-subalgebra of $\mathbf{U}$ generated by $E_{i}^{(t)}$, $F_{i}^{(t)},\left[\begin{array}{c}i ; a \\ t\end{array}\right], J_{\mu}$ and $K_{\mu}$, for all $i \in I, \mu \in Y$ and positive integers $a \geq t$.

Let $\mathcal{J}$ be the (2-sided) ideal of $\mathbf{U}$ generated by $\left\{J_{\mu}-1 \mid \mu \in Y\right\}$. The specialization at $\pi=-1$ of the algebra $\mathbf{U} / \mathcal{J}$ is naturally identified with a quantum group associated to the Cartan datum $(I, \cdot)(\mathrm{cf}$. [Lu4]). The specialization at $\pi=1$ of the algebra $\mathbf{U}$, denoted by $\left.\mathbf{U}\right|_{\pi=1}$, is a variant of this quantum group, with some extra (harmless) central elements $J_{\mu}$. Specialization at $\pi=1$ for essentially reduces our results to those of Lusztig [Lu4].

The specialization at $\pi=-1$ of the superalgebra $\mathbf{U} / \mathcal{J}$ is identified with a quantum supergroup associated to the super Cartan datum $(I, \cdot)$ considered in the literature; cf. [Y1, BKM]. The specialization at $\pi=-1$ of $\mathbf{U}$, denoted by $\left.\mathbf{U}\right|_{\pi=-1}$, will 
also be referred to as a quantum supergroup of type $(I, \cdot)$, and the extra generators $J_{i}$ allow us to formulate integrable modules $V(\lambda)$ for all $\lambda \in X^{+}$, which was not possible before.

\subsection{Hopf Structure}

An important facet of the covering quantum group is that it has the structure of a braided Hopf algebra. There are many possible choices for the coproduct, as one would expect from the theory of quantum groups. However, the covering quantum group has even more natural choices for the coproducts; see [C, §2.4]. We make $' \mathbf{U} \otimes{ }^{\prime} \mathbf{U}$ (respectively, $\mathbf{U} \otimes \mathbf{U}$ ) a $\mathbb{Q}(q)^{\pi}$-algebra via the product

$$
(a \otimes b)(c \otimes d)=\pi^{p(b) p(c)} a c \otimes b d .
$$

The main coproduct we shall consider is the following.

Lemma 3.8. There is a unique algebra homomorphism $\Delta:{ }^{\prime} \mathbf{U} \rightarrow{ }^{\prime} \mathbf{U} \otimes{ }^{\prime} \mathbf{U}$ (resp. $\Delta: \mathbf{U} \rightarrow \mathbf{U} \otimes \mathbf{U}$ ) where ${ }^{\prime} \mathbf{U} \otimes \prime \mathbf{U}$ (resp. $\mathbf{U} \otimes \mathbf{U}$ ) is regarded as a superalgebra in the standard way, defined by

$$
\begin{aligned}
\Delta\left(E_{i}\right) & =E_{i} \otimes \tilde{K}_{-i}+1 \otimes E_{i} \quad(i \in I), \\
\Delta\left(F_{i}\right) & =F_{i} \otimes 1+\tilde{J}_{i} \tilde{K}_{i} \otimes F_{i} \quad(i \in I), \\
\Delta\left(K_{\mu}\right) & =K_{\mu} \otimes K_{\mu} \quad(\mu \in Y), \\
\Delta\left(J_{\mu}\right) & =J_{\mu} \otimes J_{\mu} \quad(\mu \in Y) .
\end{aligned}
$$

Proof. The relations (3.1)-(3.3) are trivial to verify. For (3.4), we have

$$
\begin{aligned}
& \Delta\left(E_{i}\right) \Delta\left(F_{j}\right)=E_{i} F_{j} \otimes \tilde{K}_{-i}+\tilde{J}_{j} \tilde{K}_{j} \otimes E_{i} F_{j}+\pi^{p(i) p(j)} F_{j} \otimes E_{i}+E_{i} \tilde{J}_{j} \tilde{K}_{j} \otimes \tilde{K}_{-i} F_{j}, \\
& \Delta\left(F_{j}\right) \Delta\left(E_{i}\right)=F_{j} E_{i} \otimes \tilde{K}_{-i}+\tilde{J}_{j} \tilde{K}_{j} \otimes F_{j} E_{i}+F_{j} \otimes E_{i}+\pi^{p(i) p(j)} \tilde{J}_{j} \tilde{K}_{j} E_{i} \otimes F_{j} \tilde{K}_{-i} .
\end{aligned}
$$


So using the fact that $E_{i} \tilde{J}_{j} \tilde{K}_{j} \otimes \tilde{K}_{-i} F_{j}=\tilde{J}_{j} \tilde{K}_{j} E_{i} \otimes F_{j} \tilde{K}_{-i}$, we have

$$
\begin{aligned}
\Delta\left(E_{i}\right) & \Delta\left(F_{j}\right)-\pi^{p(i) p(j)} \Delta\left(F_{j}\right) \Delta\left(E_{i}\right) \\
& =\left(E_{i} F_{j}-\pi^{p(i) p(j)} F_{j} E_{i}\right) \otimes \tilde{K}_{-i}+\tilde{J}_{j} \tilde{K}_{j} \otimes\left(E_{i} F_{j}-\pi^{p(i) p(j)} F_{j} E_{i}\right) \\
& =\delta_{i, j}\left(\frac{\tilde{J}_{i} \tilde{K}_{i}-\tilde{K}_{-i}}{\pi_{i} q_{i}-q_{i}^{-1}}\right) \otimes \tilde{K}_{-i}+\tilde{J}_{i} \tilde{K}_{i} \otimes\left(\delta_{i, j} \frac{\tilde{J}_{i} \tilde{K}_{i}-\tilde{K}_{-i}}{\pi_{i} q_{i}-q_{i}^{-1}}\right) \\
& =\delta_{i, j} \frac{\Delta\left(\tilde{J}_{i}\right) \Delta\left(\tilde{K}_{i}\right)-\Delta\left(\tilde{K}_{-i}\right)}{\pi_{i} q_{i}-q_{i}^{-1}} .
\end{aligned}
$$

Finally, define maps $j^{ \pm}: ' \mathbf{f} \otimes{ }^{\prime} \mathbf{f} \rightarrow^{\prime} \mathbf{U} \otimes{ }^{\prime} \mathbf{U}$ given by

$$
j^{+}(x \otimes y)=x^{+} \otimes \tilde{K}_{-|x|} y^{+}, \quad j^{-}(x \otimes y)=x^{-} \tilde{J}_{|y|} \tilde{K}_{|y|} \otimes y^{-} .
$$

Then by construction, these maps are algebra homomorphisms, and satisfy

$$
j^{+} r(x)=\Delta\left(x^{+}\right), \quad j^{-} \bar{r}(x)=\Delta\left(x^{-}\right) .
$$

Since $r, \bar{r}$ factor through $\mathbf{f}$, so do $j^{+} \bar{r}$ and $j^{-} r$ implying that

$$
f\left(\Delta\left(E_{i}\right)\right)=f\left(\Delta\left(F_{i}\right)\right)=0
$$

for all $f\left(\theta_{i}: i \in I\right) \in \mathcal{I}$.

The coproduct $\Delta$ is coassociative, and the verification is the same as in the nonsuper case. Furthermore, we observe that $\Delta\left({ }_{\mathbb{A}} \mathbf{U}\right) \subset{ }_{\mathbb{A}} \mathbf{U} \otimes_{\mathbb{A}} \mathbf{U}$.

One of the alternate coproducts we could consider is given by

$$
\begin{aligned}
\Delta^{\prime}\left(E_{i}\right) & =E_{i} \otimes \tilde{K}_{-i}+\tilde{J}_{i} \otimes E_{i} \quad(i \in I), \\
\Delta^{\prime}\left(F_{i}\right) & =F_{i} \otimes 1+\tilde{K}_{i} \otimes F_{i} \quad(i \in I), \\
\Delta^{\prime}\left(K_{\mu}\right) & =K_{\mu} \otimes K_{\mu} \quad(\mu \in Y), \\
\Delta^{\prime}\left(J_{\mu}\right) & =J_{\mu} \otimes J_{\mu} \quad(\mu \in Y) .
\end{aligned}
$$

Let $\Delta^{\omega}=(\omega \otimes \omega) \circ \Delta \circ \omega^{-1}$. Also, let ${ }^{t} \Delta$ be the composition of $\Delta$ with the automorphism $\tau: \mathbf{U} \otimes \mathbf{U} \rightarrow \mathbf{U} \otimes \mathbf{U}$ given by $x \otimes y \mapsto \pi^{p(x) p(y)} y \otimes x$. Note that $\omega \otimes \omega$ commutes with $\tau$, so ${ }^{t} \Delta^{\omega}$ is well-defined. Then unravelling the definitions, we get the following lemma. 
Lemma 3.9. We have the identification

$$
\Delta^{\prime}={ }^{t} \Delta^{\omega}
$$

From the identities $j^{+} r(x)=\Delta\left(x^{+}\right)$and $j^{-} \bar{r}(x)=\Delta\left(x^{-}\right)$, we deduce that

$$
\begin{aligned}
\Delta\left(x^{+}\right) & =\sum x_{1}^{+} \otimes \tilde{K}_{-\left|x_{1}\right|} x_{2}^{+}, \\
\Delta\left(x^{-}\right) & =\sum \pi^{p\left(x_{1}\right) p\left(x_{2}\right)}(\pi q)^{-\left|x_{1}\right| \cdot\left|x_{2}\right|} x_{2}^{-} \tilde{J}_{\left|x_{1}\right|} \tilde{K}_{\left|x_{1}\right|} \otimes x_{1}^{-}
\end{aligned}
$$

for $r(x)=\sum x_{1} \otimes x_{2}$. In particular, this yields the formulas

$$
\begin{aligned}
& \Delta\left(E_{i}^{(p)}\right)=\sum_{p^{\prime}+p^{\prime \prime}=p} q_{i}^{p^{\prime} p^{\prime \prime}} E_{i}^{\left(p^{\prime}\right)} \otimes \tilde{K}_{i}^{-p^{\prime}} E_{i}^{\left(p^{\prime \prime}\right)} \\
& \Delta\left(F_{i}^{(p)}\right)=\sum_{p^{\prime}+p^{\prime \prime}=p}\left(\pi_{i} q_{i}\right)^{-p^{\prime} p^{\prime \prime}} \tilde{J}_{i}^{p^{\prime \prime}} F_{i}^{\left(p^{\prime}\right)} \tilde{K}_{i}^{p^{\prime \prime}} \otimes F_{i}^{\left(p^{\prime \prime}\right)} .
\end{aligned}
$$

There is a unique algebra homomorphism $\varsigma: \mathbf{U} \rightarrow \mathbb{Q}(q)^{\pi}$ satisfying $\varsigma\left(E_{i}\right)=$ $\varsigma\left(F_{i}\right)=0$ and $\varsigma\left(J_{\mu}\right)=\varsigma\left(K_{\mu}\right)=1$ for all $i, \mu$. This is the counit for $\Delta$ and makes U into a braided bi-algebra. This bi-algebra structure can be completed to a Hopf structure by defining an antipode as follows.

Lemma 3.10. Let $\nu \in \mathbb{N}[I]$. Write $\nu=\sum_{i} \nu_{i} i$ and $\nu=\sum_{a=1}^{\mathrm{h} t \nu} i_{a}$ for $i_{a} \in I$. Then we set

$$
\begin{aligned}
& c(\nu)=\nu \cdot \nu / 2-\sum_{i} \nu_{i} i \cdot i / 2 \in \mathbb{Z}, \\
& e(\nu)=\sum_{a<b} p\left(i_{a}\right) p\left(i_{b}\right) \in \mathbb{Z} .
\end{aligned}
$$

(a) There is a unique $\mathbb{Q}(q)^{\pi}$-linear map $S: \mathbf{U} \rightarrow \mathbf{U}$ such that

$$
S\left(E_{i}\right)=-E_{i} \tilde{K}_{i}, \quad S\left(F_{i}\right)=-\tilde{J}_{-i} \tilde{K}_{-i} F_{i}, \quad S\left(K_{\mu}\right)=K_{-\mu}, \quad S\left(J_{\nu}\right)=J_{-\nu}
$$

and $S(x y)=\pi^{p(x) p(y)} S(y) S(x)$ for all $x, y \in \mathbf{U}$.

(b) For any $x \in \mathbf{f}_{\nu}$, we have

$$
\begin{aligned}
& S\left(x^{+}\right)=(-1)^{\mathrm{ht} \nu} \pi^{e(\nu)} q^{c(\nu)} \varrho(x)^{+} \tilde{K}_{\nu}, \\
& S\left(x^{-}\right)=(-1)^{\mathrm{ht} \nu} \pi^{e(\nu)} \pi_{\nu} q^{-c(\nu)} \tilde{K}_{-\nu} \varrho(x)^{-}
\end{aligned}
$$


(c) There is a unique $\mathbb{Q}(q)^{\pi}$-linear map $S^{\prime}: \mathbf{U} \rightarrow \mathbf{U}$ such that

$$
S^{\prime}\left(E_{i}\right)=-\tilde{K}_{i} E_{i}, \quad S^{\prime}\left(F_{i}\right)=-F_{i} \tilde{J}_{-i} \tilde{K}_{-i}, \quad S^{\prime}\left(K_{\mu}\right)=K_{-\mu}, \quad S\left(J_{\nu}\right)=J_{-\nu}
$$

and $S^{\prime}(x y)=\pi^{p(x) p(y)} S^{\prime}(y) S^{\prime}(x)$ for all $x, y \in \mathbf{U}$.

(d) For any $x \in \mathbf{f}_{\nu}$, we have

$$
\begin{aligned}
& S^{\prime}\left(x^{+}\right)=(-1)^{\mathrm{h} t \nu} \pi^{e(\nu)} q^{-c(\nu)} \tilde{K}_{\nu} \varrho(x)^{+} \\
& S^{\prime}\left(x^{-}\right)=(-1)^{\mathrm{h} t \nu} \pi^{e(\nu)} \pi_{\nu} q^{c(\nu)} \varrho(x)^{-} \tilde{K}_{-\nu}
\end{aligned}
$$

(e) We have $S S^{\prime}=S^{\prime} S=1$.

(f) If $x \in \mathbf{f}_{\nu}$, then $S\left(x^{+}\right)=q^{-f(\nu)} S^{\prime}\left(x^{+}\right)$and $S\left(x^{-}\right)=q^{f(\nu)} S^{\prime}\left(x^{-}\right)$where $f(\nu)=$ $\sum_{i} \nu_{i} i \cdot i$

The map $S$ (resp. $S^{\prime}$ ) is called the antipode (resp. the skew-antipode) of U. Note that

$$
\begin{aligned}
S\left(E_{i}^{(n)}\right) & =(-1)^{n}\left(\pi_{i} q_{i}^{2}\right)^{\left(\begin{array}{c}
n \\
2
\end{array}\right)} E_{i}^{(n)} \tilde{K}_{n i}, \\
S^{\prime}\left(E_{i}^{(n)}\right) & =(-1)^{n}\left(\pi_{i} q_{i}^{2}\right)^{-\left(\begin{array}{c}
n \\
2
\end{array}\right)} \tilde{K}_{n i} E_{i}^{(n)}, \\
S\left(F_{i}^{(n)}\right) & =(-1)^{n}\left(\pi_{i} q_{i}^{2}\right)^{-\left(\begin{array}{c}
n \\
2
\end{array}\right)} \tilde{K}_{-n i} F_{i}^{(n)}, \\
S^{\prime}\left(F_{i}^{(n)}\right) & =(-1)^{n}\left(\pi_{i} q_{i}^{2}\right)^{\left(\begin{array}{c}
n \\
2
\end{array}\right)} F_{i}^{(n)} \tilde{K}_{-n i} .
\end{aligned}
$$

\subsection{Triangular Decomposition}

If $M^{\prime}, M$ are two ' $\mathbf{U}$-modules, then $M^{\prime} \otimes M$ is naturally a ' $\mathbf{U} \otimes{ }^{\prime} \mathbf{U}$-module; hence by restriction to ' $\mathbf{U}$ under $\Delta$, it is a ' $\mathbf{U}$-module.

Lemma 3.11. Let $\lambda \in X$. There is a unique 'U-module structure on the $\mathbb{Q}(q)^{\pi}$ module'f such that for any homogeneous $z \in$ 'f , and $\mu \in Y$ and any $i \in I$, we have

$$
K_{\mu} \cdot z=q^{\langle\mu, \lambda-|z|\rangle} z, \quad J_{\mu} \cdot z=\pi^{\langle\mu, \lambda-|z|\rangle} z, \quad F_{i} \cdot z=\theta_{i} z, \quad E_{i} \cdot 1=0
$$


Proof. The uniqueness is immediate. To prove the existence, define

$$
E_{i} \cdot z=\frac{-q_{i}^{\langle i, \lambda\rangle} r_{i}(z)+\pi_{i}^{p(z)-p(i)}\left(\pi_{i} q_{i}\right)^{\left\langle i, \lambda-|z|+i^{\prime}\right\rangle}{ }_{i} r(z)}{\pi_{i} q_{i}-q_{i}^{-1}}
$$

Note that this is essentially the formula prescribed by Proposition 3.5. A straightforward computation shows that this, along with the desired formulas for the $F$ and $K$ actions define a 'U-module structure on 'f.

We denote this ' $\mathbf{U}$-module by $M^{\prime}(\lambda)$ (which is a free $\mathbb{Q}(q)^{\pi}$-module). Similarly, to an element $\lambda \in X$, we associate a unique ' $\mathbf{U}$-module structure on 'f such that for any homogeneous $z \in$ 'f, any $\mu \in Y$ and any $i \in I$ we have

$$
K_{\mu} \cdot z=q^{\langle\mu,-\lambda+|z|\rangle} z, \quad J_{\mu} \cdot z=\pi^{\langle\mu,-\lambda+|z|\rangle} z, \quad E_{i} \cdot z=\theta_{i} z, \quad F_{i} \cdot 1=0 .
$$

We denote this ${ }^{\prime} \mathbf{U}$-module by ${ }^{\omega} M^{\prime}(\lambda)$ (which is again a free $\mathbb{Q}(q)^{\pi}$-module). We form the 'U-module ${ }^{\omega} M^{\prime}\left(\lambda^{\prime}\right) \otimes_{\mathbb{Q}(q)^{\pi}} M^{\prime}(\lambda)$ for $\lambda, \lambda^{\prime} \in X$; we denote the unit element of 'f $=M^{\prime}(\lambda)$ by 1 and that of ' $\mathbf{f}={ }^{\omega} M^{\prime}\left(\lambda^{\prime}\right)$ by $1^{\prime}$. Thus, we have the canonical element $1^{\prime} \otimes 1 \in^{\omega} M^{\prime}\left(\lambda^{\prime}\right) \otimes_{\mathbb{Q}(q)^{\pi}} M^{\prime}(\lambda)$. We emphasize that ${ }^{\omega} M^{\prime}\left(\lambda^{\prime}\right) \otimes_{\mathbb{Q}(q)^{\pi}} M^{\prime}(\lambda)$ is again free as a $\mathbb{Q}(q)^{\pi}$-module.

Proposition 3.12. Let $\mathbf{U}^{0}$ be the associative $\mathbb{Q}(q)^{\pi}$-algebra with 1 defined by the generators $K_{\mu}, J_{\mu}(\mu \in Y)$ and the relations (3.1). Then $\mathbf{U}^{0}$ is isomorphic to the group algebra of $Y \times(Y / 2 Y)$ over $\mathbb{Q}(q)^{\pi}$. Moreover,

(a) The $\mathbb{Q}(q)^{\pi}$-linear map ${ }^{\prime} \mathbf{f} \otimes \mathbf{U}^{0} \otimes{ }^{\prime} \mathbf{f} \rightarrow{ }^{\prime} \mathbf{U}$ given by $u \otimes J_{\nu} K_{\mu} \otimes w \mapsto u^{-} J_{\nu} K_{\mu} w^{+}$ is an isomorphism.

(b) The $\mathbb{Q}(q)^{\pi}$-linear map ${ }^{\prime} \mathbf{f} \otimes \mathbf{U}^{0} \otimes{ }^{\prime} \mathbf{f} \rightarrow{ }^{\prime} \mathbf{U}$ given by $u \otimes J_{\nu} K_{\mu} \otimes w \mapsto u^{+} J_{\nu} K_{\mu} w^{-}$ is an isomorphism.

Proof. Note that (b) follows from (a) by applying $\omega$. As a $\mathbb{Q}(q)^{\pi}$-module, ' $\mathbf{U}$ is spanned by words in the $E_{i}, F_{i}, K_{\mu}$, and $J_{\mu}$. By using the defining relations, we can rewrite any word as a linear combination of words where the $F_{i}$ come before the $J_{\mu}$ and $K_{\mu}$, which come before the $E_{i}$, thus the given map is surjective. 
To prove the map is injective, let $\lambda, \lambda^{\prime} \in X$, and consider the module ${ }^{\omega} M^{\prime}\left(\lambda^{\prime}\right) \otimes_{\mathbb{Q}(q)^{\pi}}$ $M^{\prime}(\lambda)$ described before. There is a $\mathbb{Q}(q)^{\pi}$-linear map $\Phi:{ }^{\prime} \mathbf{U} \rightarrow{ }^{\omega} M^{\prime}\left(\lambda^{\prime}\right) \otimes_{\mathbb{Q}(q)^{\pi}}$ $M^{\prime}(\lambda)$ given by $\Phi(u)=u \cdot 1^{\prime} \otimes 1$. Pick a $\mathbb{Q}(q)^{\pi}$-basis of 'f consisting of homogeneous elements containing 1. Assume that in ' $\mathbf{U}$ there is some relation of the form $\sum_{b^{\prime}, \mu, b} c_{b^{\prime}, \mu, b} b^{-} J_{\nu} K_{\mu} b^{+}=0$ and let $N$ be the largest integer such that ht $\left|b^{\prime}\right|=N$ and $c_{b^{\prime}, \mu, b} \neq 0$ for some $\mu, b$.

Then

$$
0=\Phi\left(\sum_{b^{\prime}, \mu, \nu, b} c_{b^{\prime}, \mu, \nu, b} b^{-} J_{\nu} K_{\mu} b^{+}\right)=\sum_{b^{\prime}, \mu, \nu, b} c_{b^{\prime}, \mu, \nu, b} \Delta\left(b^{\prime-} J_{\nu} K_{\mu} b^{+}\right) \cdot 1 \otimes 1 .
$$

Now

$$
\begin{aligned}
& \Delta\left(b^{\prime-}\right)=\sum_{b_{1}^{\prime}, b_{2}^{\prime}} g^{\prime}\left(b^{\prime}, b_{1}^{\prime}, b_{2}^{\prime}\right) b_{1}^{\prime-} \otimes \tilde{K}_{-\left|b_{1}^{\prime}\right|} b_{2}^{\prime-} \\
& \Delta\left(b^{+}\right)=\sum_{b_{1}, b_{2}} g\left(b, b_{1}, b_{2}\right) b_{1}^{+} \tilde{J}_{\left|b_{2}\right|} \tilde{K}_{\left|b_{2}\right|} \otimes b_{2}^{+},
\end{aligned}
$$

so we have

$$
\begin{aligned}
& 0=\sum \pi^{p\left(b_{2}^{\prime}\right) p\left(b_{1}\right)} c_{b^{\prime}, \mu, \nu, b} g\left(b, b_{1}, b_{2}\right) g^{\prime}\left(b^{\prime}, b_{1}^{\prime}, b_{2}^{\prime}\right) b_{1}^{--} \times \\
& \times J_{\nu} K_{\mu} b_{1}^{+} \tilde{J}_{\left|b_{2}\right|} \tilde{K}_{\left|b_{2}\right|} \cdot 1^{\prime} \otimes \tilde{K}_{-\left|b_{1}^{\prime}\right|} b_{2}^{\prime-} J_{\nu} K_{\mu} b_{2}^{+} \cdot 1
\end{aligned}
$$

If $b_{2} \neq 1$, then $b_{2}^{+} \cdot 1=0$ so we must have $b_{2}=1$ and thus $b_{1}=b$. Therefore the expression reduces to

$$
0=\sum \pi^{p\left(b_{2}^{\prime}\right) p(b)} c_{b^{\prime}, \mu, \nu, b} g^{\prime}\left(b^{\prime}, b_{1}^{\prime}, b_{2}^{\prime}\right) b_{1}^{\prime-} J_{\nu} K_{\mu} b^{+} \cdot 1^{\prime} \otimes \tilde{K}_{-\mid b_{1}^{\prime}} b_{2}^{\prime-} J_{\nu} K_{\mu} \cdot 1
$$

By the definition of the module structure, this becomes

$$
0=\sum \pi^{p\left(b_{2}^{\prime}\right) p(b)} c_{b^{\prime}, \mu, \nu, b} g^{\prime}\left(b^{\prime}, b_{1}^{\prime}, b_{2}^{\prime}\right) \pi^{\left\langle\nu, \lambda-\lambda^{\prime}+|b|\right\rangle} q^{\left\langle\mu, \lambda-\lambda^{\prime}+|b|\right\rangle} b_{1}^{\prime-} \cdot b \otimes \tilde{K}_{-\left|b_{1}^{\prime}\right|} b_{2}^{\prime}
$$

We can now project this equality onto the summand ${ }^{\omega} M^{\prime}\left(\lambda^{\prime}\right) \otimes_{\mathbb{Q}(q)^{\pi}} \mathbf{f}_{\nu}$ where ht $\nu=N$. Then by construction, $\left|b_{2}^{\prime}\right| \leq|b|$ and ht $\left|b_{2}^{\prime}\right|=N$. Since $c_{b^{\prime}, \mu, b}=0$ if ht $\left|b^{\prime}\right|>N$, we must have $|b|=\left|b_{2}^{\prime}\right|$ and thus $b^{\prime}=b_{2}^{\prime}, b_{1}^{\prime}=1$, so

$$
\sum \pi^{p\left(b^{\prime}\right) p(b)} c_{b^{\prime}, \mu, \nu, b} \pi^{\left\langle\nu, \lambda-\lambda^{\prime}+|b|\right\rangle} q^{\left\langle\mu, \lambda-\lambda^{\prime}+|b|\right\rangle} b \otimes b^{\prime}=0
$$


It follows that

$$
\sum_{\nu, \mu} c_{b^{\prime}, \mu, \nu, b} \pi^{\left\langle\nu, \lambda-\lambda^{\prime}+|b|\right\rangle} q^{\left\langle\mu, \lambda-\lambda^{\prime}+|b|\right\rangle}=0
$$

for all choices of $\lambda, \lambda^{\prime}, \mu, b$ and $b^{\prime}$ with ht $\left|b^{\prime}\right|=N$. Therefore $c_{b^{\prime}, \mu, \nu, b}=0$ for any $b^{\prime}$ with ht $\left|b^{\prime}\right|=N$, contradicting the choice of $N$.

Corollary 3.13. (a) The $\mathbb{Q}(q)^{\pi}$-linear map $\mathbf{f} \otimes \mathbf{U}^{0} \otimes \mathbf{f} \rightarrow \mathbf{U}$ given by $u \otimes J_{\nu} K_{\mu} \otimes w \mapsto$ $u^{-} J_{\nu} K_{\mu} w^{+}$is an isomorphism.

(b) The $\mathbb{Q}(q)^{\pi}$-linear map $\mathbf{f} \otimes \mathbf{U}^{0} \otimes \mathbf{f} \rightarrow \mathbf{U}$ given by $u \otimes K_{\mu} \otimes w \mapsto u^{+} J_{\nu} K_{\mu} w^{-}$is an isomorphism.

Proof. Once again (b) follows from (a) by applying the involution $\omega$. Let $J_{ \pm}$be the two-sided ideal of ' $\mathbf{U}$ generated by $\mathcal{I}^{ \pm}=\left\{x^{ \pm}: x \in \mathcal{I}\right\}$. Then $\mathbf{U}=\frac{{ }^{\prime} \mathbf{U}}{J_{+}+J_{-}}$. Now from Proposition 3.5 iterated, we see that

$$
\left({ }^{\prime} \mathbf{U}^{+}\right) \mathcal{I}^{-} \subseteq \mathcal{I}^{-} \mathbf{U}^{0}\left({ }^{\prime} \mathbf{U}^{+}\right) ; \quad \mathcal{I}^{+}\left({ }^{\prime} \mathbf{U}^{-}\right) \subseteq\left({ }^{\prime} \mathbf{U}^{-}\right) \mathbf{U}^{0} \mathcal{I}^{+}
$$

Using the triangular decomposition of ' $\mathbf{U}$, we have $J_{-}={ }^{\prime} \mathbf{U I}^{-\prime} \mathbf{U} \subseteq \mathcal{I}^{-} \mathbf{U}^{0}\left({ }^{\prime} \mathbf{U}^{+}\right) \subseteq$ $J_{-}$, hence $J_{-}=\mathcal{I}^{-} \mathbf{U}^{0}\left({ }^{\prime} \mathbf{U}^{+}\right)$. Similarly, $J_{+}=\left({ }^{\prime} \mathbf{U}^{-}\right) \mathbf{U}^{0} \mathcal{I}^{+}$. Therefore,

$$
\mathbf{U}=\frac{{ }^{\prime} \mathbf{U}^{-} \otimes \mathbf{U}^{0} \otimes{ }^{\prime} \mathbf{U}^{+}}{\prime \mathbf{U}^{-} \otimes \mathbf{U}^{0} \otimes \mathcal{I}^{+}+\mathcal{I}^{-} \otimes \mathbf{U}^{0} \otimes \mathbf{U}^{+}}=\frac{{ }^{\prime} \mathbf{U}^{-}}{\mathcal{I}^{-}} \otimes \mathbf{U}^{0} \otimes \frac{{ }^{\prime} \mathbf{U}^{+}}{\mathcal{I}^{+}},
$$

from which (a) follows.

Corollary 3.14. The maps ${ }^{ \pm}: \mathbf{f} \rightarrow \mathbf{U}^{ \pm}, x \mapsto x^{ \pm}$, are $\mathbb{Q}(q)^{\pi}$-algebra isomorphisms, and $\mathbf{U}^{0} \rightarrow \mathbf{U}$ is a $\mathbb{Q}(q)^{\pi}$-algebra embedding.

For $\nu \in \mathbb{N}[I]$, we shall denote the image $\mathbf{f}_{\nu}^{ \pm}$by $\mathbf{U}_{\nu}^{ \pm}$.

Proposition 3.15. Let $x \in \mathbf{f}_{\nu}$ where $\nu \in \mathbb{N}[I]$ is nonzero.

(a) If $x^{+} F_{i}=\pi_{i}^{p(x)} F_{i} x^{+}$for all $i \in I$ then $x=0$.

(b) If $x^{-} E_{i}=\pi_{i}^{p(x)} E_{i} x^{-}$for all $i \in I$ then $x=0$.

Proof. It follows from Proposition 3.5 and the linear independence of $r_{i}(x)^{+} \tilde{J}_{i} \tilde{K}_{i}$ (respectively, the linear independence of $\tilde{J}_{i} \tilde{K}_{-i}{ }_{i} r(x)^{+}$) that $r_{i}(x)^{+}={ }_{i} r(x)^{+}=0$ for all $i$. Hence $x=0$ by Lemma 2.10 . 


\subsection{The twistor automorphism of $\mathrm{U}$}

Let $\mathbf{T}$ be the group algebra of the group $\mathbb{Z}[I] \times Y$; that is, the $\mathbb{Q}(q, \mathbf{t})^{\pi}$-algebra with generators $T_{\mu}, \Upsilon_{\nu}$, for $\mu \in Y$ and $\nu \in \mathbb{Z}[I]$, and relations

$$
T_{\mu} T_{\mu^{\prime}}=T_{\mu+\mu^{\prime}}, \quad \Upsilon_{\nu} \Upsilon_{\nu^{\prime}}=\Upsilon_{\nu+\nu^{\prime}}, \quad T_{\mu} \Upsilon_{\nu}=\Upsilon_{\nu} T_{\mu}, \quad T_{0}=\Upsilon_{0}=1
$$

We define an action of $\mathbf{T}$ on $\mathbf{U}[\mathbf{t}]$ by

$$
T_{\mu} \cdot x=\mathbf{t}^{\left\langle\mu, \eta^{\prime}\right\rangle} x, \quad \Upsilon_{\nu} \cdot x=\mathbf{t}^{\phi(\nu, \eta)} x \text { for all } x \in \mathbf{U}[\mathbf{t}]_{\eta} .
$$

Then we form the semi-direct $\mathbb{Q}(q, \mathbf{t})^{\pi}$-algebra $\widehat{\mathbf{U}}[\mathbf{t}]=\mathbf{T} \ltimes \mathbf{U}[\mathbf{t}]$ with respect to the above action of $\mathbf{T}$; that is, $T x T^{-1}=T \cdot x$ for all $T \in \mathbf{T}$ and $x \in \mathbf{U}[\mathbf{t}]$. By specialization, we obtain a $\mathbb{Q}(q, \mathbf{t})^{\pi}$-algebra $\widehat{\mathbf{U}}[\mathbf{t}]$, which is called the extended covering quantum group. We will extend the tilde notation to $\tilde{T}_{\nu}=T_{\tilde{\nu}}$. Now we will define a version of the twistor isomorphism on this algebra. We will abuse notation and also use $\mathfrak{X}$ to denote this map.

Proposition 3.16. There is a $\mathbb{Q}(\mathbf{t})$-algebra automorphism $\mathfrak{X}$ on $\widehat{\mathbf{U}}[\mathbf{t}]$ such that

$$
\begin{gathered}
\mathfrak{X}\left(E_{i}\right)=\mathbf{t}_{i}^{-1} \Upsilon_{i}^{-1} \tilde{T}_{i} E_{i}, \quad \mathfrak{X}\left(F_{i}\right)=F_{i} \Upsilon_{i}, \quad \mathfrak{X}\left(K_{\nu}\right)=T_{-\nu} K_{\nu}, \quad \mathfrak{X}\left(J_{\nu}\right)=T_{\nu}^{2} J_{\nu}, \\
\mathfrak{X}\left(T_{\nu}\right)=T_{\nu}, \quad \mathfrak{X}\left(\Upsilon_{\nu}\right)=\Upsilon_{\nu}, \quad \mathfrak{X}(q)=\mathbf{t}^{-1} q, \quad \widehat{\Psi}(\pi)=-\pi .
\end{gathered}
$$

The automorphism $\mathfrak{X}$ will be called the twistor on $\widehat{\mathbf{U}}[\mathbf{t}]$.

Proof. We first show that such a map is well defined by showing that relations (3.1)(3.6) and (3.10) are satisfied by the images of the generators. The relations (3.1)-(3.3) and (3.10) are straightforward to verify, and we leave this to the reader.

Let us verify (3.4). On one hand, we have

$$
\begin{aligned}
\mathfrak{X}\left(E_{i}\right) & \mathfrak{X}\left(F_{j}\right)-\mathfrak{X}(\pi)^{p(i) p(j)} \mathfrak{X}\left(F_{j}\right) \widehat{\Psi}\left(E_{i}\right) \\
& =\mathbf{t}_{i}^{-1} \Upsilon_{i}^{-1} \tilde{T}_{i} E_{i} F_{j} \Upsilon_{j}-(-\pi)^{p(i) p(j)} F_{j} \Upsilon_{j} \mathbf{t}_{i}^{-1} \Upsilon_{i}^{-1} \tilde{T}_{i} E_{i} \\
& =\mathbf{t}^{-d_{i}+d_{j}-\phi(j, i)} \Upsilon_{i}^{-1} \Upsilon_{j} \tilde{T}_{i}\left(E_{i} F_{j}-\mathbf{t}^{i \cdot j+\phi(j, i)-\phi(i, j)}(-\pi)^{p(i) p(j)} F_{j} E_{i}\right) \\
& =\mathbf{t}^{-d_{i}+d_{j}-\phi(j, i)} \Upsilon_{i} \Upsilon_{j}^{-1} \tilde{T}_{i}\left(E_{i} F_{j}-\pi^{p(i) p(j)} F_{j} E_{i}\right),
\end{aligned}
$$


where the last equality follows from Definition $2.18(\mathrm{c})$ and $\mathbf{t}^{2}=-1$. On the other hand,

$$
\begin{aligned}
\delta_{i j} \frac{\mathfrak{X}\left(\tilde{J}_{i}\right) \mathfrak{X}\left(\tilde{K}_{i}\right)-\mathfrak{X}\left(\tilde{K}_{-i}\right)}{\mathfrak{X}\left(\pi_{i}\right) \mathfrak{X}\left(q_{i}\right)-\mathfrak{X}\left(q_{i}\right)^{-1}} & =\delta_{i j} \frac{\tilde{T}_{i} \tilde{J}_{i} \tilde{K}_{i}-\tilde{T}_{i} \tilde{K}_{-i}}{(-\mathbf{t})^{-d_{i}} \pi_{i} q_{i}-\mathbf{t}_{i} q_{i}^{-1}} \\
& =\delta_{i j} \mathbf{t}_{i}^{-1} \tilde{T}_{i} \frac{\tilde{J}_{i} \tilde{K}_{i}-\tilde{K}_{-i}}{\pi_{i} q_{i}-q_{i}^{-1}} .
\end{aligned}
$$

Then comparing (3.12) and (3.13), we see that they are equal for all $i, j \in I$, whence $(3.4)$.

It remains to check the Serre relations (3.5) and (3.6). As these computations are entirely similar, let us prove (3.6). As in the proof of Theorem 2.20, we have

$$
\begin{aligned}
& \sum_{k=0}^{b_{i j}}(-1)^{k}(-\pi)^{\left(\begin{array}{c}
k \\
2
\end{array}\right) p(i)+k p(i) p(j)}\left[\begin{array}{c}
b_{i j} \\
k
\end{array}\right]_{\mathbf{t}^{-1} q_{i},-\pi_{i}}\left(F_{i} \Upsilon_{i}\right)^{b_{i j}-k}\left(F_{j} \Upsilon_{j}\right)\left(F_{i} \Upsilon_{i}\right)^{k} \\
& =\left(\sum_{k=0}^{b_{i j}}(-1)^{k} \pi^{\left(\begin{array}{c}
k \\
2
\end{array}\right) p(i)+k p(i) p(j)}\left[\begin{array}{c}
b_{i j} \\
k
\end{array}\right]_{q_{i}, \pi_{i}} F_{i}^{b_{i j}-k} F_{j} F_{i}^{k}\right) \mathbf{t}^{\left(\begin{array}{c}
b_{i j} \\
2
\end{array}\right)+i \cdot j+b_{i, j} \phi\left(i, j^{\prime}\right)} \Upsilon_{b_{i j} i+j}=0 .
\end{aligned}
$$

The proposition is proved.

Remark 3.17. An earlier construction of Lanzmann [Lan] described a similar transformation in finite type, which explained various similarities between the representation theories of $\mathfrak{o s p}(1 \mid 2 n)$ and $\mathfrak{s o}(1+2 n)$. The twistor isomorphism can be viewed as a variant of that construction, which has the advantage of working for non-finite type as well.

Now note that the maps $\theta_{i} \mapsto \theta_{i}^{-}=F_{i}$ and $\theta_{i} \mapsto \theta_{i}^{+}=E_{i}$ extend to embeddings $\mathbf{f}[\mathbf{t}] \rightarrow \widehat{\mathbf{U}}^{-}$and $\mathbf{f}[\mathbf{t}] \rightarrow \widehat{\mathbf{U}}^{+}$. These embeddings relate the twistor maps as follows: for $\nu=\sum_{i \in I} \nu_{i} i \in \mathbb{N}[I]$ and $x \in \mathbf{f}[\mathbf{t}]_{\nu}$,

$$
\mathfrak{X}\left(x^{-}\right)=\mathfrak{X}(x)^{-} \Upsilon_{-\nu}, \quad \mathfrak{X}\left(x^{+}\right)=\mathbf{t}^{\bullet(\nu)+\sum 2 d_{i} \nu_{i}} \mathfrak{X}(x)^{+} \tilde{T}_{|x|} \Upsilon_{|x|} .
$$

\subsection{The categories $\mathcal{C}$ and $\mathcal{O}$}

By a representation of the algebra $\mathbf{U}$ we mean $\mathbb{Q}(q)^{\pi}$-module on which $\mathbf{U}$ acts. Note we have a direct sum decomposition of the $\mathbb{Q}(q)^{\pi}$-module $\mathbb{Q}(q)^{\pi}=\epsilon_{+} \mathbb{Q}(q) \oplus \epsilon_{-} \mathbb{Q}(q)$, 
where $\pi$ acts as 1 on $\epsilon_{+} \mathbb{Q}(q)$ and as -1 on $\epsilon_{-} \mathbb{Q}(q)$.

We define the category $\mathcal{C}$ (of weight $\mathbf{U}$-modules) as follows. An object of $\mathcal{C}$ is a $\mathbb{Z} / 2 \mathbb{Z}$-graded $\mathbf{U}$-module $M=M^{0} \oplus M^{1}$, compatible with the $\mathbb{Z} / 2 \mathbb{Z}$-grading on $\mathbf{U}$, with a given weight space decomposition

$$
M=\bigoplus_{\lambda \in X} M_{\lambda}, \quad M_{\lambda}=\left\{m \in M \mid K_{\mu} m=q^{\langle\mu, \lambda\rangle} m, J_{\mu} m=\pi^{\langle\mu, \lambda\rangle} m, \forall \mu \in Y\right\},
$$

such that $M_{\lambda}=M_{\lambda}^{0} \oplus M_{\lambda}^{1}$ where $M_{\lambda}^{0}=M_{\lambda} \cap M^{0}$ and $M_{\lambda}^{1}=M_{\lambda} \cap M^{1}$. The $\mathbb{Z} / 2 \mathbb{Z}$ graded structure is only particularly relevant to tensor products, and will generally be suppressed when irrelevant.

We have the following $\mathbb{Q}(q)^{\pi}$-module decomposition for each weight space: $M^{\lambda}=$ $\epsilon_{-} M_{\lambda} \oplus \epsilon_{+} M_{\lambda}$; accordingly, we have $M=\left.\left.M\right|_{\pi=1} \oplus M\right|_{\pi=-1}$ as $\mathbf{U}$-modules, where $\left.M\right|_{\pi= \pm 1}:=\oplus_{\lambda \in X} \epsilon_{ \pm} M_{\lambda}$ is an $\mathbf{U}$-module on which $\pi$ acts as \pm 1 , i.e. a $\left.\mathbf{U}\right|_{\pi= \pm 1}$-module. Hence the category $\mathcal{C}$ decomposes into a direct $\operatorname{sum} \mathcal{C}=\mathcal{C}_{+} \oplus \mathcal{C}_{-}$, where $\mathcal{C}_{ \pm}$can be identified with categories of weight modules over the specializations $\left.\mathbf{U}\right|_{\pi= \pm 1}$.

Lemma 3.18. A simple $\mathbf{U}$-module is a simple module of either $\left.\mathbf{U}\right|_{\pi=1}$ or $\left.\mathbf{U}\right|_{\pi=-1}$.

Let $M \in \mathcal{C}$ and let $m \in M_{\lambda}$. The formulas below follow from Lemma 3.7.

(a) $E_{i}^{(N)} F_{i}^{(M)} m=\sum_{t} \pi_{i}^{M N-\left(\begin{array}{c}(+1) \\ 2\end{array}\right)}\left[\begin{array}{c}N-M+\langle i, \lambda\rangle \\ t\end{array}\right]_{i} F_{i}^{(M-t)} E_{i}^{(N-t)} m$;

(b) $F_{i}^{(M)} E_{i}^{(N)} m=\sum_{t} \pi_{i}^{(M-t)(N-t)-t^{2}}\left[\begin{array}{c}M-N-\langle i, \lambda\rangle \\ t\end{array}\right]_{i} E_{i}^{(N-t)} F_{i}^{(M-t)} m$;

(c) $F_{i}^{(M)} E_{j}^{(N)} m=E_{j}^{(N)} F_{i}^{(M)} m$, for $i \neq j$;

(d) $\quad\left[\begin{array}{c}i ; a \\ t\end{array}\right] m=\left[\begin{array}{c}\langle i, \lambda\rangle+a \\ t\end{array}\right]_{i} m$.

A tensor product of $\mathbf{U}$-modules $M \otimes N$ is naturally a $\mathbf{U} \otimes \mathbf{U}$-module with the obvious grading under the action $(x \otimes y)(m \otimes n)=\pi^{p(y) p(m)} x m \otimes y n$. In particular, the tensor product of modules is naturally a $\mathbf{U}$-module under the coproduct. To wit, given $\mathbf{U}$-modules $M$ and $M^{\prime}$, we set $M \otimes M^{\prime}=M \otimes_{\mathbb{Q}(q)^{\pi}} M^{\prime}$ as $\mathbb{Q}(q)^{\pi}$-modules with the 
U-action given by $u \cdot m \otimes m^{\prime}=\Delta(u) m \otimes m^{\prime}$. Similarly, we set $M \otimes^{\prime} M^{\prime}=M \otimes_{\mathbb{Q}(q)^{\pi}} M^{\prime}$ with the $\mathbf{U}$-action given by $u \cdot m \otimes m^{\prime}=\Delta^{\prime}(u) m \otimes m^{\prime}$.

We see that $\mathcal{C}$ is closed under both tensor products. To any $M \in \mathcal{C}$, we can define a new $\mathbf{U}$-module structure via $u \cdot m=\omega(u) m$; we denote this module by ${ }^{\omega} M$. By definition, note that ${ }^{\omega} M_{\lambda}=M_{-\lambda}$. We can also see how the $\omega$-twist affects tensor products of modules.

Lemma 3.19. For any $\mathbf{U}$-modules $M, M^{\prime}$, there is a $\mathbf{U}$-module isomorphism

$$
\tau:{ }^{\omega}\left(M \otimes{ }^{\prime} M^{\prime}\right) \rightarrow{ }^{\omega} M^{\prime} \otimes{ }^{\omega} M
$$

given by $\tau(x \otimes y): \pi^{p(y) p(x)} y \otimes x$.

Proof. Recall that $\Delta^{\prime}={ }^{t} \Delta^{\omega}$. Let $u \in \mathbf{U}$ and suppose $\Delta^{\prime}(\omega(u))=\sum u_{1} \otimes u_{2}$ with $u_{1}, u_{2}$ homogeneous. Then

$$
\begin{aligned}
\tau\left(\Delta^{\prime}(\omega(u))(x \otimes y)\right) & =\tau\left(\sum \pi^{p(x) p\left(u_{2}\right)} u_{1} x \otimes u_{2} y\right) \\
& =\sum \pi^{p(x) p\left(u_{2}\right)+p\left(u_{1} x\right) p\left(u_{2} y\right)} \sum u_{2} y \otimes u_{1} x \\
& =\left(\sum \pi^{p\left(u_{2}\right) p\left(u_{1}\right)} \omega^{-1}\left(u_{2}\right) \otimes \omega^{-1}\left(u_{1}\right)\right) \tau(x \otimes y) \\
& =\tau \circ\left(\omega^{-1} \otimes \omega^{-1}\right) \circ \Delta_{4}(\omega(u)) \tau(x \otimes y) \\
& =\Delta(u) \tau(x \otimes y) .
\end{aligned}
$$

Let $\lambda \in X$. Then there is a unique $\mathbf{U}$-module structure on $\mathbf{f}$ such that for any $y \in \mathbf{f}, \mu \in Y$ and $i \in I$ we have $K_{\mu} y=q^{\langle\mu, \lambda-|y|\rangle} y, J_{\nu} y=\pi^{\langle\nu, \lambda-|y|\rangle} y, F_{i} y=\theta_{i} y$, and $E_{i} 1=0$. As in the non-super case, this follows readily from the triangular decomposition. This module will be called a Verma module and denoted by $M(\lambda)$. The parity grading on $\mathbf{f}$ induces a parity grading on $M(\lambda)$ where $p(1)=0$. As before, we have a U-module decomposition $M(\lambda)=\left.\left.M(\lambda)\right|_{\pi=1} \oplus M(\lambda)\right|_{\pi=-1}$, where $\left.M(\lambda)\right|_{\pi= \pm 1}$ can be identified as the Verma module of $\left.\mathbf{U}\right|_{\pi= \pm 1}$ (which is a $\mathbb{Q}(q)$ )-vector space). 
For any $M \in \mathcal{C}$ and an element $m \in M_{\lambda}$ such that $E_{i} m=0$ for all $i$, there is a unique $\mathbf{U}$-homomorphism $M(\lambda) \rightarrow M$ via $1 \mapsto m$. This can be proved as in [Lu4, 3.4.6] using now Lemma 3.7.

Let $\mathcal{O}$ be the full subcategory of $\mathcal{C}$ such that for any $M$ in $\mathcal{O}$ and $m \in M$, there exists an $n \geq 0$ such that $x^{+} m=0$ for all $x \in \mathbf{f}_{\nu}$ with ht $\nu \geq n$. Note that $M(\lambda)$ and its quotient $\mathbf{U}$-modules belong to $\mathcal{O}$.

An object $M \in \mathcal{C}$ is said to be integrable if for any $m \in M$ and any $i \in I$, there exists $n_{0} \geq 1$ such that $E_{i}^{(n)} m=F_{i}^{(n)} m=0$ for all $n \geq n_{0}$. Let $\mathcal{C}_{\text {int }}$ be the full subcategory of $\mathcal{C}$ whose objects are the integrable $\mathbf{U}$-modules.

For $M, M^{\prime}, M^{\prime \prime} \in \mathcal{C}_{\text {int }}$, we have ${ }^{\omega} M, M^{\prime} \otimes M^{\prime \prime} \in \mathcal{C}_{\text {int }}$. The proof of the following lemma proceeds as in the non-super case; see [Lu4, Lemma 3.5.3].

Lemma 3.20. For $\left(a_{i}\right),\left(b_{i}\right) \in \mathbb{N}^{I}$ and $\lambda \in X$, let $M$ be the quotient of $\mathbf{U}$ by the left ideal generated by the elements $F_{i}^{a_{i}+1}, E_{i}^{b_{i}+1}, K_{\mu}-q^{\langle\mu, \lambda\rangle}$ with $\mu \in Y$, and $J_{\nu}-\pi^{\langle\nu, \lambda\rangle}$ with $\nu \in Y$. Then $M$ is an integrable $\mathbf{U}$-module.

The proof of the following proposition proceeds as in the non-super case; see [Lu4, Proposition 3.5.4 and 23.3.11].

Proposition 3.21. If $u \in \mathrm{U}$ such that $u$ acts as zero on every integrable module, then $u=0$.

Proposition 3.22. Let $\lambda \in X^{+}$.

(a) Let $\mathcal{T}$ be the left ideal of $\mathbf{f}$ generated by the elements $\theta_{i}^{\langle i, \lambda\rangle+1}$ for all $i \in I$. Then $\mathcal{T}$ is a $\mathbf{U}$-submodule of the Verma module $M(\lambda)$.

(b) The quotient $\mathbf{U}$-module $V(\lambda):=M(\lambda) / \mathcal{T}$ is integrable.

The proof is as in the non-super case [Lu4, Proposition 3.5.6]. As usual $V(\lambda)=$ $\left.\left.V(\lambda)\right|_{\pi=1} \oplus V(\lambda)\right|_{\pi=-1}$, and $\mathcal{T}=\left.\left.\mathcal{T}\right|_{\pi=1} \oplus \mathcal{T}\right|_{\pi=-1} ;$ moreover we have the identification $\left.V(\lambda)\right|_{\pi= \pm 1}=\left.M(\lambda)\right|_{\pi= \pm 1} /\left.\mathcal{T}\right|_{\pi= \pm 1}$. 
We denote the image of 1 in $V(\lambda)$ by $\eta_{\lambda}$. This module has an induced parity grading from the associated Verma module, so in particular $p\left(\eta_{\lambda}\right)=0$. When considering the image of 1 in the module ${ }^{\omega} V(\lambda)$, we will denote this vector by $\xi_{\lambda}$.

Proposition 3.23. Let $M$ be an object of $\mathcal{C}_{\text {int }}$ and let $m \in M^{\lambda}$ be a non-zero vector such that $E_{i} m=0$ for all $i$. Then $\lambda \in X^{+}$and there is a unique morphism (in $\mathcal{C}_{\text {int }}$ ) $t^{\prime}: V(\lambda) \rightarrow M$ sending $\eta_{\lambda}$ to $m$.

Proof. The proof is as in the non-super case [Lu4, Proposition 3.5.8].

An important variant of these modules are the integral forms. Since $M(\lambda)=\mathbf{f}$ as a vector space, it is automatically endowed with an $\mathbb{A}$-submodule ${ }_{\mathbb{A}} M(\lambda)={ }_{\mathbb{A}} \mathbf{f}$. We call this the integral form of $M(\lambda)$. We also have integral forms for the irreducible modules: we call the $\mathbb{A}$-submodule ${ }_{\mathbb{A}} V(\lambda)={ }_{\mathbb{A}} \mathbf{U} \xi_{-\lambda}$ (resp. ${ }_{\mathbb{A}}^{\omega} V(\lambda)={ }_{\mathbb{A}} \mathbf{U} \xi_{-\lambda}$ ) the integral form of $V(\lambda)$ (resp. ${ }^{\omega} V(\lambda)$ ).

The following particular family of $\mathbf{U}$-modules will be of critical importance later on. We define

$$
N\left(\lambda, \lambda^{\prime}\right)=V(\lambda) \otimes{ }^{\omega} V\left(\lambda^{\prime}\right), \quad \mathbb{A}_{\mathbb{A}} N\left(\lambda, \lambda^{\prime}\right)={ }_{\mathbb{A}} V(\lambda) \otimes_{\mathbb{A}}\left({ }_{\mathbb{A}}^{\omega} V\left(\lambda^{\prime}\right)\right) .
$$

Then $N\left(\lambda, \lambda^{\prime}\right)\left(\right.$ resp. ${ }_{\mathbb{A}} N\left(\lambda, \lambda^{\prime}\right)$ ) is a $\mathbf{U}$-module (resp. ${ }_{\mathbb{A}} \mathbf{U}$-module) under the coproduct $\Delta$. When we wish to view these spaces as modules with respect to the coproduct $\Delta^{\prime}$, we will use the notation $N^{\prime}\left(\lambda, \lambda^{\prime}\right)$ (respectively, $\mathbb{A} N^{\prime}\left(\lambda, \lambda^{\prime}\right)$ ). The module structures on $N\left(\lambda, \lambda^{\prime}\right)$ and $N^{\prime}\left(\lambda, \lambda^{\prime}\right)$ are quite closely related, as demonstrated by the following lemma.

Lemma 3.24. The linear isomorphism $N\left(\lambda, \lambda^{\prime}\right) \rightarrow N^{\prime}\left(\lambda, \lambda^{\prime}\right)$ given by

$$
x \otimes y \mapsto \pi^{\langle\tilde{\nu}, \lambda\rangle} x \otimes y \quad \text { for all } x \in V(\lambda), y \in{ }^{\omega} V\left(\lambda^{\prime}\right)_{\nu^{\prime}-\lambda^{\prime}}, \quad \nu \in \mathbb{N}[I],
$$

is a $\mathbf{U}$-module isomorphism.

Proof. This is elementary to verify using the definitions. 
Since $\omega$ is not an involution, we could also consider $\omega^{2} M$ for any module $M$. However, twisting the action on $V(\lambda)$ by $\omega^{2}$ does not yield any new module. To wit, we have the following lemma.

Lemma 3.25. The $\mathbb{Q}(q)^{\pi}$-linear isomorphism $\omega^{2}: V(\lambda) \rightarrow \omega^{2} V(\lambda)$ given by

$$
\omega^{2}(x)=\pi_{\nu} \pi^{\langle\tilde{\nu}, \lambda\rangle} x, \quad x \in V(\lambda)_{\lambda-\nu^{\prime}}, \nu \in \mathbb{N}[I]
$$

is a $\mathbf{U}$-module isomorphism.

Proof. For any homogeneous $u \in \mathbf{U}_{\mu}, \omega^{2}(u)=\pi_{\mu} \tilde{J}_{\mu} u$. Then for $x \in V(\lambda)_{\lambda-\nu^{\prime}}$ with $\nu \in \mathbb{N}[I]$, we have $\omega^{2}(u x)=\pi_{\mu+\nu} \pi^{\langle\tilde{\mu}+\tilde{\nu}, \lambda\rangle} u x=\omega^{2}(u) \omega^{2}(x)$.

\subsection{The twistor and modules}

Recall the notation $M[\mathbf{t}]=\mathbb{Q}(q, \mathbf{t})^{\pi} \otimes_{\mathbb{Q}(q)^{\pi}} M$. Let $M \in \mathcal{C}$. Then $M[\mathbf{t}]$ carries a natural action of $\mathbf{T}$ by setting

$$
T_{\nu} \Upsilon_{\mu} m=\mathbf{t}^{\langle\nu, \lambda\rangle+\phi(\mu, \lambda)} m, \quad m \in M[\mathbf{t}]_{\lambda}
$$

In particular, $M[\mathbf{t}]$ is a $\hat{\mathbf{U}}[\mathbf{t}]-$ module.

Lemma 3.26. Let $\mathfrak{X}_{\lambda}: M(\lambda)[\mathbf{t}] \rightarrow M(\lambda)[\mathbf{t}]$ be the map defined by

$$
\mathfrak{X}_{\lambda}(x)=\mathbf{t}^{-\phi(\nu, \lambda)} \mathfrak{X}(x)
$$

for homogeneous $x \in \mathbf{f}[\mathbf{t}]_{\nu}$. Then $\mathfrak{X}_{\lambda}(u x)=\mathfrak{X}(u) \mathfrak{X}_{\lambda}(x)$ for all $u \in \widehat{\mathbf{U}}$ and $x \in \mathbf{f}[\mathbf{t}]$.

Proof. It is enough to prove the lemma for the generators of $\widehat{\mathbf{U}}$. First note that $\mathfrak{X}_{\lambda}$ preserves weight spaces, and so the lemma is clear for $T_{\mu}$ and $\Upsilon_{\mu}$; it is also clear that $\mathfrak{X}(q m)=\mathfrak{X}(q) \mathfrak{X}_{\lambda}(x)$ and $\mathfrak{X}(\pi x)=\mathfrak{X}(\pi) \mathfrak{X}_{\lambda}(x)$, whence $\mathfrak{X}_{\lambda}\left(K_{\mu} x\right)=\mathfrak{X}\left(K_{\mu}\right) \mathfrak{X}_{\lambda}(x)$ and $\mathfrak{X}_{\lambda}\left(J_{\mu} x\right)=\mathfrak{X}\left(J_{\mu}\right) \mathfrak{X}_{\lambda}(x)$. It remains to check for $u=E_{i}$ and $u=F_{i}$.

Let $x \in \mathbf{f}[\mathbf{t}]_{\nu}$. Then for $u=F_{i}$ we have

$$
\mathfrak{X}_{\lambda}\left(F_{i} x\right)=\mathfrak{X}_{\lambda}\left(\theta_{i} x\right)=\mathbf{t}^{\phi\left(i, \nu^{\prime}\right)-\phi(\nu+i, \lambda)} \theta_{i} \mathfrak{X}(x)=F_{i} \Upsilon_{-i} \mathfrak{X}_{\lambda}(x)=\mathfrak{X}\left(F_{i}\right) \mathfrak{X}_{\lambda}(x)
$$


On the other hand, for $u=E_{i}$ we have

$$
\begin{aligned}
& \mathfrak{X}_{\lambda}\left(E_{i} x\right)=\mathfrak{X}_{\lambda}\left(\frac{\pi_{i}^{p(\nu)-p(i)} r_{i}(x)\left(\pi_{i} q_{i}\right)^{\langle i, \lambda\rangle}-q_{i}^{-\left\langle i, \lambda-\nu^{\prime}+i^{\prime}\right\rangle}{ }_{i} r(x)}{\pi_{i} q_{i}-q_{i}^{-1}}\right) \\
& =\mathbf{t}^{-d_{i}-\phi(\nu-i, \lambda)} \frac{\mathbf{t}_{i}^{2 p(i) p(\nu-i)+\langle i, \lambda\rangle} \pi_{i}^{p(\nu)-p(i)} \mathfrak{X}\left(r_{i}(x)\right)\left(\pi_{i} q_{i}\right)^{\langle i, \lambda\rangle}}{\pi_{i} q_{i}-q_{i}^{-1}} \\
& \quad-\mathbf{t}^{-d_{i}-\phi(\nu-i, \lambda)} \frac{\mathbf{t}_{i}^{\left\langle i, \lambda-\nu+i^{\prime}\right\rangle} q_{i}^{-\left\langle i, \lambda-\nu+i^{\prime}\right\rangle} \mathfrak{X}\left({ }_{i} r(x)\right)}{\pi_{i} q_{i}-q_{i}^{-1}} \\
& =\mathbf{t}^{-d_{i}-\phi(\nu-i, \lambda)+d_{i}\left\langle i, \lambda-\nu+i^{\prime}\right\rangle-\phi\left(i, \nu^{\prime}-i^{\prime}\right)} \frac{\mathbf{t}_{i}^{\star} \pi_{i}^{p(\nu)-p(i)} r_{i}(\mathfrak{X}(x))\left(\pi_{i} q_{i}\right)^{\langle i, \lambda\rangle}-q_{i}^{-\left\langle i, \lambda-\nu+i^{\prime}\right\rangle}{ }_{i} r(\mathfrak{X}(x))}{\pi_{i} q_{i}-q_{i}^{-1}}
\end{aligned}
$$

where

$$
\star=2 p(i) p(\nu-i)+d_{i}\langle i, \lambda\rangle-\phi(\nu-i, i)-d_{i}\left\langle i, \lambda-\nu+i^{\prime}\right\rangle+\phi\left(i, \nu^{\prime}-i^{\prime}\right) .
$$

One checks that $\star \in 4 \mathbb{Z}$ and therefore,

$$
\begin{aligned}
\mathfrak{X}_{\lambda}\left(E_{i} x\right) & =\mathbf{t}^{-d_{i}-\phi(\nu-i, \lambda)+d_{i}\left\langle i, \lambda-\nu+i^{\prime}\right\rangle-\phi\left(i, \nu^{\prime}-i^{\prime}\right)} E_{i} \mathfrak{X}(x) \\
& =\mathbf{t}_{i}^{\langle i, \lambda-\nu\rangle} \mathbf{t}^{\phi(i, \lambda-\nu)} \mathbf{t}^{-\phi(\nu, \lambda)+2 d_{i}} E_{i} \mathfrak{X}(x) \\
& =\mathbf{t}_{i}^{2} E_{i} \tilde{T}_{i} \Upsilon_{i} \mathfrak{X}_{\lambda}(x)=\mathfrak{X}\left(E_{i}\right) \mathfrak{X}_{\lambda}(x) .
\end{aligned}
$$

Recall that $V(\lambda)=\mathbf{f} /\left(\theta_{i}^{\langle i, \lambda\rangle+1}: i \in I\right)$ as vector spaces. Then $V(\lambda)[\mathbf{t}]=$ $\mathbf{f}[\mathbf{t}] /\left(\theta_{i}^{\langle i, \lambda\rangle+1}: i \in I\right)$. Since we further have

$$
\mathfrak{X}_{\lambda}\left(\left(\theta_{i}^{\langle i, \lambda\rangle+1}: i \in I\right)\right)=\left(\theta_{i}^{\langle i, \lambda\rangle+1}: i \in I\right)
$$

we see that $\mathfrak{X}_{\lambda}$ induces a $\mathbb{Q}(\mathbf{t})$-linear isomorphism

$$
\mathfrak{X}_{\lambda}: V(\lambda)[\mathbf{t}] \rightarrow V(\lambda)[\mathbf{t}] .
$$

Lemma 3.27. There is a $\mathbb{Q}(\mathbf{t})$-linear map $\mathfrak{X}_{\lambda}: V(\lambda)[\mathbf{t}] \rightarrow V(\lambda)[\mathbf{t}]$ which satisfies $\mathfrak{X}_{\lambda}\left(\eta_{\lambda}\right)=\eta_{\lambda}$ and $\mathfrak{X}_{\lambda}(u m)=\mathfrak{X}(u) \mathfrak{X}_{\lambda}(m)$ for all $u \in \widehat{\mathbf{U}}$ and $m \in V(\lambda)[\mathbf{t}]$.

There is an important immediate corollary. By a character of an $X$-graded vector space over a field $\mathbb{F}$, we mean the formal expression $\operatorname{ch}_{\mathbb{F}} M=\sum_{\lambda \in X} \operatorname{dim}_{\mathbb{F}} M_{\lambda} e^{\lambda}$, where $e^{\lambda}$ are the basis elements of the group ring $\mathbb{Z}[X]$. 
Corollary 3.28. Recall that $W$ is the Weyl group associated to $(I, \cdot)$. Denote by $\rho \in X$ such that $\langle i, \rho\rangle=1$ for all $i \in I$. Then for every $\lambda \in X^{+}$:

$$
\left.\operatorname{ch}_{\mathbb{Q}(q)} V(\lambda)\right|_{\pi= \pm 1}=\frac{\sum_{w \in W}(-1)^{\ell(w)} e^{w(\lambda+\rho)-\rho}}{\sum_{w \in W}(-1)^{\ell(w)} e^{w(\rho)-\rho}} .
$$

Moreover, the character of $\mathbf{U}^{-}$is given by

$$
\left.\operatorname{ch}_{\mathbb{Q}(q)} \mathbf{U}^{-}\right|_{ \pm 1}=\frac{1}{\sum_{w \in W}(-1)^{\ell(w)} e^{w(\rho)-\rho}}
$$

Proof. Since the $\pi=1$ case is known, it suffices to prove the character formula for $\pi=-1$. In this case, both results follow from the twistor isomorphisms. Indeed, it is clear that these characters hold when dimensions are taken with respect to $\mathbb{Q}(q, \mathbf{t})$, but each weight space is just a scalar extension of a $\mathbb{Q}(q)$-vector space, so of course dimensions stay the same.

Remark 3.29. A particular consequence of this character formula is that the weight spaces of $V(\lambda)$ are always free $\mathbb{Q}(q)^{\pi}$ modules for each $\lambda \in X^{+}$.

There is a version of the map $\mathfrak{X}_{\lambda}$ which can be defined on the $\omega$-twisted module structure. Set $\mathfrak{X}^{\prime}=\omega^{-1} \circ \mathfrak{X} \circ \omega$; we note that for $i \in I, \nu_{1}, \nu_{2}, \nu_{3} \in Y$, and $\mu \in \mathbb{Z}[I]$, we have

$$
\mathfrak{X}^{\prime}\left(E_{i}\right)=\tilde{T}_{-i} \mathfrak{X}\left(E_{i}\right), \quad \mathfrak{X}^{\prime}\left(F_{i}\right)=\mathfrak{X}\left(F_{i}\right) \tilde{T}_{i}, \quad \mathfrak{X}^{\prime}\left(J_{\nu_{1}} K_{\nu_{2}} T_{\nu_{3}} \Upsilon_{\mu}\right)=\mathfrak{X}\left(J_{\nu_{1}} K_{\nu_{2}} T_{\nu_{3}} \Upsilon_{\mu}\right) .
$$

In particular,

$$
\begin{gathered}
\Delta\left(\mathfrak{X}\left(E_{i}\right)\right)=\mathfrak{X}\left(E_{i}\right) \otimes \mathfrak{X}^{\prime}\left(\tilde{K}_{i}\right) \Upsilon_{i}+\mathbf{t}_{i}^{2}\left(\Upsilon_{i} \otimes \mathfrak{X}^{\prime}\left(E_{i}\right)\right)\left(\tilde{T}_{i} \otimes \tilde{T}_{i}\right), \\
\Delta\left(\mathfrak{X}\left(F_{i}\right)\right)=\mathfrak{X}\left(F_{i}\right) \otimes \Upsilon_{-i}+\left(\Upsilon_{-i} \mathfrak{X}\left(\tilde{J}_{i} \tilde{K}_{i}\right) \otimes \mathfrak{X}^{\prime}\left(F_{i}\right)\right)\left(\tilde{T}_{-i} \otimes \tilde{T}_{-i}\right) .
\end{gathered}
$$

Lemma 3.30. There is a $\mathbb{Q}(\mathbf{t})$-linear map $\mathfrak{X}_{-\lambda}:{ }^{\omega} V(\lambda)[\mathbf{t}] \rightarrow{ }^{\omega} V(\lambda)[\mathbf{t}]$ which satisfies

$$
\mathfrak{X}_{\lambda}\left(\xi_{-\lambda}\right)=\xi_{-\lambda}, \quad T w_{-\lambda}(u m)=\mathfrak{X}^{\prime}(u) \mathfrak{X}_{-\lambda}(m)
$$

for all $u \in \widehat{\mathbf{U}}$ and $m \in{ }^{\omega} V(\lambda)[\mathbf{t}]$. 
Proof. View $m \in{ }^{\omega} V(\lambda)[\mathbf{t}]$ as an element of $V(\lambda)[\mathbf{t}]$ as an element of $V(\lambda)$ and set $\mathfrak{X}_{-\lambda}(m)=\mathfrak{X}_{\lambda}(m)$. Then

$$
\mathfrak{X}_{-\lambda}(u \cdot m)=\mathfrak{X}_{\lambda}(\omega(u) m)=\mathfrak{X}(\omega(u)) \mathfrak{X}_{\lambda}(m)=\mathfrak{X}^{\prime}(u) \cdot \mathfrak{X}_{-\lambda}(m) .
$$

We can now define a version of the twistor on the modules $N\left(\lambda, \lambda^{\prime}\right)$. To do this, we first require the existence of a certain normalization function. Let $\Lambda_{\lambda}=$ $\left\{\lambda-\nu^{\prime} \mid \nu \in \mathbb{N}[I]\right\}$ and $\mathrm{V}_{\lambda}=\left\{\nu^{\prime}-\lambda \mid \nu \in \mathbb{N}[I]\right\}$. and for $\zeta \in \Lambda_{\lambda}\left(\right.$ resp. $\left.\zeta \in \mathrm{V}_{\lambda}\right)$ such that $\zeta=\lambda-\nu(\operatorname{resp} . \zeta=\nu-\lambda)$, set $p(\zeta)=p(\nu)$.

Lemma 3.31. There is a function $\varkappa=\varkappa_{\lambda, \lambda^{\prime}}: \Lambda_{\lambda} \times \mathrm{V}_{\lambda^{\prime}} \rightarrow \mathbb{Z} / 4 \mathbb{Z}$ satisfying

1. $\varkappa\left(\zeta-i^{\prime}, \zeta^{\prime}\right)=\varkappa\left(\zeta, \zeta^{\prime}\right)-\phi\left(i, \zeta^{\prime}\right)$

2. $\varkappa\left(\zeta, \zeta^{\prime}+i\right)=\varkappa\left(\zeta, \zeta^{\prime}\right)+\phi(i, \zeta)+2 d_{i}+\left\langle\tilde{i}, \zeta+\zeta^{\prime}\right\rangle+2 p(\zeta) p(i)$.

3. $\varkappa\left(\lambda,-\lambda^{\prime}\right)=0$

Proof. Let $\mu, \nu \in \mathbb{N}[I]$ and write $\zeta=\lambda-\nu, \zeta^{\prime}=\mu-\lambda^{\prime}$. Set

$$
\varkappa\left(\lambda,-\lambda^{\prime}\right)=0, \quad \varkappa\left(\lambda-\nu^{\prime},-\lambda^{\prime}\right)=\phi\left(\nu, \lambda^{\prime}\right)
$$

Then we have defined $\varkappa\left(\lambda-\nu^{\prime}, \mu^{\prime}-\lambda^{\prime}\right)$ for ht $(\mu)=0$; now assume $\mathrm{ht}(\mu)>0$. Define

$$
\begin{array}{r}
\varkappa\left(\lambda-\nu^{\prime}, i^{\prime}+\mu^{\prime}-\lambda^{\prime}\right)=\varkappa \\
\left(\lambda-\nu^{\prime}, \mu^{\prime}-\lambda^{\prime}\right)+\phi\left(i, \lambda-\nu^{\prime}\right)+2 d_{i} \\
+\left\langle\tilde{i}, \lambda-\nu^{\prime}+\mu^{\prime}-\lambda^{\prime}\right\rangle+2 p(\nu) p(i) .
\end{array}
$$

It is straightforward to check that this definition does not depend on the choice of $i$; that is, if $i+\mu=j+\hat{\mu}$ for some $\hat{\mu} \in \mathbb{N}[I]$, then $\varkappa\left(\lambda-\nu^{\prime}, i^{\prime}+\mu^{\prime}-\lambda^{\prime}\right)=$ $\varkappa\left(\lambda-\nu^{\prime}, j^{\prime}+\hat{\mu}^{\prime}-\lambda^{\prime}\right)$.

Now we need to check that $\varkappa\left(\lambda-\nu^{\prime}, \mu^{\prime}-\lambda\right)$ satisfies (1) and (2). By construction, we see that (1) and (2) hold for $\operatorname{ht}(\nu+\mu)=0$, so assume that $\operatorname{ht}(\nu+\mu)>0$. By 
construction, (2) holds and so it suffices to check (1). Write $\mu=\mu_{1}+j$ for some $j \in I$ and $\nu_{1} \in \mathbb{N}[I]$. Then by induction we compute that

$$
\begin{gathered}
\varkappa\left(\lambda-\nu^{\prime}-i^{\prime}, \mu^{\prime}-\lambda\right)=\varkappa\left(\lambda-\nu^{\prime}-i^{\prime}, \mu_{2}^{\prime}-\lambda\right)+\phi\left(j, \lambda-\nu^{\prime}-i^{\prime}\right)+2 d_{j} \\
\quad+\left\langle\tilde{j}, \lambda-\nu^{\prime}-i^{\prime}+\mu_{2}^{\prime}-\lambda^{\prime}\right\rangle+2 p(\nu+i) p(i) \\
=\varkappa\left(\lambda-\nu^{\prime}, \mu_{2}^{\prime}-\lambda\right)-\phi\left(i, \mu_{2}^{\prime}-\lambda^{\prime}\right)+\phi\left(j, \lambda-\nu^{\prime}-i^{\prime}\right)+2 d_{j} \\
\quad+\left\langle\tilde{j}, \lambda-\nu^{\prime}-i^{\prime}+\mu_{2}^{\prime}-\lambda^{\prime}\right\rangle+2 p(\nu+i) p(i) \\
=\varkappa\left(\lambda-\nu^{\prime}, \mu_{2}^{\prime}-\lambda\right)+2 p(\nu) p(j)+\phi\left(j, \lambda-\nu^{\prime}\right)+2 d_{j} \\
\quad+\left\langle\tilde{j}, \lambda-\nu^{\prime}+\mu_{2}^{\prime}-\lambda^{\prime}\right\rangle-i \cdot j+2 p(i) p(j)-\phi(j, i)-\phi\left(i, \mu_{2}^{\prime}-\lambda^{\prime}\right) \\
=\varkappa\left(\lambda-\nu^{\prime}, \mu^{\prime}-\lambda\right)-i \cdot j+2 p(i) p(j)-\phi(j, i)-\phi\left(i, \mu_{2}^{\prime}-\lambda^{\prime}\right)
\end{gathered}
$$

Now $-i \cdot j+2 p(i) p(j)-\phi(j, i)=-\phi(i, j)$, and thus we see that

$$
\varkappa\left(\lambda-\nu^{\prime}-i^{\prime}, \mu^{\prime}-\lambda\right)=\varkappa\left(\lambda-\nu^{\prime}, \mu^{\prime}-\lambda\right)-\phi\left(i, \mu^{\prime}-\lambda^{\prime}\right) .
$$

Proposition 3.32. The $\mathbb{Q}(\mathbf{t})$-linear map $\mathfrak{X}_{\lambda, \lambda^{\prime}}: N\left(\lambda, \lambda^{\prime}\right)[\mathbf{t}] \rightarrow N\left(\lambda, \lambda^{\prime}\right)[\mathbf{t}]$ defined by

$$
\mathfrak{X}_{\lambda, \lambda^{\prime}}(v \otimes w)=\mathbf{t}^{\varkappa(|v|,|w|)} \mathfrak{X}_{\lambda}(v) \otimes \mathfrak{X}_{-\lambda^{\prime}}(w),
$$

where $|v|=\lambda-\nu^{\prime}$ and $|w|=\lambda^{\prime}-\mu^{\prime}$ for $\nu, \mu \in \mathbb{N}[I]$. Then $\mathfrak{X}_{\lambda, \lambda^{\prime}}(\Delta(u) v \otimes w)=$ $\Delta(\mathfrak{X}(u)) \mathfrak{X}_{\lambda, \lambda^{\prime}}(v \otimes w)$.

Proof. It is enough to check when $u$ is a generator. If $u$ is $K_{\nu}, J_{\nu}, T_{\nu}$, or $\Upsilon_{\nu}$ then this is trivial, so it remains to check when $u=E_{i}$ or $u=F_{i}$. Let $v \otimes w \in N\left(\lambda, \lambda^{\prime}\right)$ with $|v|=\zeta$ and $|w|=\zeta^{\prime}$. First, we compute that

$$
\begin{aligned}
\Delta\left(\mathfrak{X}\left(E_{i}\right)\right) \mathfrak{X}_{\lambda}(v) \otimes \mathfrak{X}_{-\lambda^{\prime}}(w)= & \mathbf{t}^{\phi\left(i, \zeta^{\prime}\right)} \mathfrak{X}_{\lambda}\left(E_{i} v\right) \otimes \mathfrak{X}_{-\lambda^{\prime}}\left(\tilde{K}_{-i} \cdot w\right) \\
& +\pi^{p(i) p(v)} \mathbf{t}^{2 d_{i}+d_{i}\left\langle i, \zeta+\zeta^{\prime}\right\rangle+\phi(i, \zeta)} \mathfrak{X}_{\lambda}(v) \otimes \mathfrak{X}_{-\lambda^{\prime}}\left(E_{i} \cdot w\right) \\
\Delta\left(\mathfrak{X}\left(F_{i}\right)\right) \mathfrak{X}_{\lambda}(v) \otimes \mathfrak{X}_{-\lambda^{\prime}}(w)= & \mathbf{t}^{-\phi\left(i, \zeta^{\prime}\right)} \mathfrak{X}_{\lambda}\left(F_{i} v\right) \otimes \mathfrak{X}_{-\lambda^{\prime}}(w) \\
& +\pi^{p(i) p(v)} \mathbf{t}^{-d_{i}\left\langle i, \zeta-\zeta^{\prime}\right\rangle-\phi(i, \zeta)} \mathfrak{X}_{\lambda}\left(\tilde{J}_{i} \tilde{K}_{i} v\right) \otimes \mathfrak{X}_{-\lambda^{\prime}}\left(F_{i} \cdot w\right)
\end{aligned}
$$


Then using (3.18), we have

$$
\begin{aligned}
& \Delta\left(\mathfrak{X}\left(E_{i}\right)\right) \mathfrak{X}_{\lambda, \lambda^{\prime}}(v \otimes w)=\mathbf{t}^{\varkappa\left(\zeta, \zeta^{\prime}\right)} \Delta\left(\mathfrak{X}\left(E_{i}\right)\right)\left(\mathfrak{X}_{\lambda}(v) \otimes \mathfrak{X}_{-\lambda^{\prime}}(w)\right) \\
& =\mathbf{t}^{\phi\left(i, \zeta^{\prime}\right)+\varkappa\left(\zeta, \zeta^{\prime}\right)} \mathfrak{X}_{\lambda}\left(E_{i} v\right) \otimes \mathfrak{X}_{-\lambda^{\prime}}\left(\tilde{K}_{-i} \cdot w\right) \\
& \quad+\pi^{p(i) p(v)} \mathbf{t}^{\varkappa\left(\zeta, \zeta^{\prime}\right)+2 d_{i}+d_{i}\left\langle i, \zeta+\zeta^{\prime}\right\rangle+\phi(i, \zeta)} \mathfrak{X}_{\lambda}(v) \otimes \mathfrak{X}_{-\lambda^{\prime}}\left(E_{i} \cdot w\right) \\
& =\mathbf{t}^{\phi\left(i, \zeta^{\prime}\right)+\varkappa\left(\zeta, \zeta^{\prime}\right)-\varkappa\left(\zeta+i, \zeta^{\prime}\right)} \mathfrak{X}_{\lambda, \lambda^{\prime}}\left(E_{i} v \otimes \tilde{K}_{-i} \cdot w\right) \\
& \quad+\pi^{p(i) p(v)} \mathbf{t}^{\varkappa\left(\zeta, \zeta^{\prime}\right)-\varkappa\left(\zeta, \zeta^{\prime}+i\right)+2 d_{i}+d_{i}\left\langle i, \zeta+\zeta^{\prime}\right\rangle+\phi(i, \zeta)} \mathfrak{X}_{\lambda, \lambda^{\prime}}\left(v \otimes E_{i} \cdot w\right) \\
& =\mathfrak{X}_{\lambda, \lambda^{\prime}}\left(E_{i} v \otimes \tilde{K}_{-i} \cdot w\right)+\pi^{p(i) p(v)} \mathbf{t}^{2 p(i) p(\zeta)} \mathfrak{X}_{\lambda, \lambda^{\prime}}\left(v \otimes E_{i} \cdot w\right) \\
& =\mathfrak{X}_{\lambda, \lambda^{\prime}}\left(\Delta\left(E_{i}\right) v \otimes w\right) .
\end{aligned}
$$

Similarly, using (3.19), we have

$$
\begin{aligned}
& \Delta\left(\mathfrak{X}\left(F_{i}\right)\right) \mathfrak{X}_{\lambda, \lambda^{\prime}}(v \otimes w)=\mathbf{t}^{\varkappa\left(\zeta, \zeta^{\prime}\right)} \Delta\left(\mathfrak{X}\left(F_{i}\right)\right)\left(\mathfrak{X}_{\lambda}(v) \otimes \mathfrak{X}_{-\lambda^{\prime}}(w)\right) \\
& =\mathbf{t}^{-\phi\left(i, \zeta^{\prime}\right)+\varkappa\left(\zeta, \zeta^{\prime}\right)} \mathfrak{X}_{\lambda}\left(F_{i} v\right) \otimes \mathfrak{X}_{-\lambda^{\prime}}(w) \\
& \quad+\pi^{p(i) p(v)} \mathbf{t}^{\varkappa\left(\zeta, \zeta^{\prime}\right)-d_{i}\left\langle i, \zeta+\zeta^{\prime}\right\rangle-\phi(i, \zeta)} \mathfrak{X}_{\lambda}\left(\tilde{J}_{i} \tilde{K}_{i} v\right) \otimes \mathfrak{X}_{-\lambda^{\prime}}\left(F_{i} \cdot w\right) \\
& =\mathbf{t}^{-\phi\left(i, \zeta^{\prime}\right)+\varkappa\left(\zeta, \zeta^{\prime}\right)-\varkappa\left(\zeta-i^{\prime}, \zeta^{\prime}\right)} \mathfrak{X}_{\lambda, \lambda^{\prime}}\left(F_{i} v \otimes w\right) \\
& \quad+\pi^{p(i) p(v)} \mathbf{t}^{\varkappa\left(\zeta, \zeta^{\prime}\right)-\varkappa\left(\zeta, \zeta^{\prime}-i^{\prime}\right)-d_{i}\left\langle i, \zeta+\zeta^{\prime}\right\rangle-\phi(i, \zeta)} \mathfrak{X}_{\lambda, \lambda^{\prime}}\left(\tilde{J}_{i} \tilde{K}_{i} v \otimes F_{i} \cdot w\right) \\
& =\mathfrak{X}_{\lambda, \lambda^{\prime}}\left(\Delta\left(F_{i}\right) v \otimes w\right)
\end{aligned}
$$

\subsection{The quasi- $\mathcal{R}$-matrix}

Consider the vector spaces

$$
\mathcal{H}_{N}=\mathbf{U}^{-} \mathbf{U}^{0}\left(\sum_{\mathrm{h} t \nu \geq N} \mathbf{U}_{\nu}^{+}\right) \otimes \mathbf{U}+\mathbf{U} \otimes \mathbf{U}^{+} \mathbf{U}^{0}\left(\sum_{\mathrm{h} t \nu \geq N} \mathbf{U}_{\nu}^{-}\right)
$$

for $N \in \mathbb{Z}_{>0}$. Note that $\mathcal{H}_{N}$ is a left ideal in $\mathbf{U} \otimes \mathbf{U}$; moreover, for any $u \in \mathbf{U} \otimes \mathbf{U}$, we can find an $r \geq 0$ such that $\mathcal{H}_{N+r} u \subset \mathcal{H}_{N}$. 
Let $(\mathbf{U} \otimes \mathbf{U})^{\wedge}$ be the inverse limit of the vector spaces $(\mathbf{U} \otimes \mathbf{U}) / \mathcal{H}_{n}$. Then the $\mathbb{Q}(q)^{\pi}$-algebra structure extends by continuity to a $\mathbb{Q}(q)^{\pi}$-algebra structure on $(\mathbf{U} \otimes \mathbf{U})^{\wedge}$, and we have the obvious algebra embedding $\mathbf{U} \otimes \mathbf{U} \rightarrow(\mathbf{U} \otimes \mathbf{U})^{\wedge}$.

Let $^{-}: \mathbf{U} \otimes \mathbf{U} \rightarrow \mathbf{U} \otimes \mathbf{U}$ be the $\mathbb{Q}$-algebra homomorphism given by ${ }^{-} \otimes^{-}$. This extends to a $\mathbb{Q}$-algebra homomorphism on the completion. Let $\bar{\Delta}: \mathbf{U} \rightarrow \mathbf{U} \otimes \mathbf{U}$ be the $\mathbb{Q}(q)^{\pi}$-algebra homomorphism given by $\bar{\Delta}(x)=\overline{\Delta(\bar{x})}$.

Theorem 3.33. (a) There is a unique family of elements $\Theta_{\nu} \in \mathbf{U}_{\nu}^{+} \otimes \mathbf{U}_{\nu}^{-}$(with $\nu \in \mathbb{N}[I])$ such that $\Theta_{0}=1 \otimes 1$ and $\Theta=\sum_{\nu} \Theta_{\nu} \in(\mathbf{U} \otimes \mathbf{U})^{\wedge}$ satisfies $\Delta(u) \Theta=$ $\Theta \bar{\Delta}(u)$ for all $u \in \mathbf{U}$ (where this identity is in $(\mathbf{U} \otimes \mathbf{U})^{\wedge}$ ).

(b) Let $B$ be a $\mathbb{Q}(q)^{\pi}$-basis of $\mathbf{f}$ such that $B_{\nu}=B \cap \mathbf{f}_{\nu}$ is a basis of $\mathbf{f}_{\nu}$ for any $\nu$. Let $\left\{b^{*} \mid b \in B_{\nu}\right\}$ be the basis of $\mathbf{f}_{\nu}$ dual to $B_{\nu}$ under $(-,-)$. We have

$$
\Theta_{\nu}=(-1)^{\mathrm{ht} \nu} \pi^{e(\nu)} \pi_{\nu} q_{\nu} \sum_{b \in B_{\nu}} b^{*+} \otimes b^{-} \in \mathbf{U}_{\nu}^{+} \otimes \mathbf{U}_{\nu}^{-},
$$

where e $(\nu)$ is defined as in Lemma 3.10.

The element $\Theta$ will be called the quasi- $\mathcal{R}$-matrix for $\mathbf{U}$.

Proof. Consider an element $\Theta \in(\mathbf{U} \otimes \mathbf{U})^{\wedge}$ of the form $\Theta=\sum_{\nu} \Theta_{\nu}$ with $\Theta_{\nu}=$ $\sum_{b, b^{\prime} \in B_{\nu}} c_{b^{\prime}, b} b^{\prime-} \otimes b^{*+}, c_{b^{\prime}, b} \in \mathbb{Q}(q)^{\pi}$. The set of $u \in \mathbf{U}$ such that $\Delta(u) \Theta=\Theta \bar{\Delta}(u)$ is clearly a subalgebra of $\mathbf{U}$ containing $\mathbf{U}^{0}$. Therefore, it is necessary and sufficient that it contains the $E_{i}$ and $F_{i}$. This amounts to showing that

$$
\begin{aligned}
& \sum_{b_{1}, b_{2} \in B_{\nu-i}} c_{b_{1}, b_{2}} E_{i} b_{1}^{*+} \otimes \tilde{K}_{-i} b_{2}^{-}+\sum_{b_{3}, b_{4} \in B_{\nu}} \pi_{i}^{p\left(b_{3}\right)} c_{b_{3}, b_{4}} b_{3}^{*+} \otimes E_{i} b_{4}^{-} \\
= & \sum_{b_{1}, b_{2} \in B_{\nu-i}} \pi_{i}^{p\left(b_{2}\right)} c_{b_{1}, b_{2}} b_{1}^{*+} E_{i} \otimes b_{2}^{-} \tilde{J}_{i} \tilde{K}_{i}+\sum_{b_{3}, b_{4} \in B_{\nu}} c_{b_{3}, b_{4}} b_{3}^{*+} \otimes b_{4}^{-} E_{i},
\end{aligned}
$$

and

$$
\begin{aligned}
& \sum_{b_{1}, b_{2} \in B_{\nu-i}} \pi_{i}^{p\left(b_{1}\right)} c_{b_{1}, b_{2}} \tilde{J}_{i} \tilde{K}_{i} b_{1}^{*+} \otimes F_{i} b_{2}^{-}+\sum_{b_{3}, b_{4} \in B_{\nu}} c_{b_{3}, b_{4}} F_{i} b_{3}^{*+} \otimes b_{4}^{-} \\
& =\sum_{b_{1}, b_{2} \in B_{\nu-i}} c_{b_{1}, b_{2}} b_{1}^{*+} \tilde{K}_{-i} \otimes b_{2}^{-} F_{i}+\sum_{b_{3}, b_{4} \in B_{\nu}} \pi_{i}^{p\left(b_{4}\right)} c_{b_{3}, b_{4}} b_{3}^{*+} F_{i} \otimes b_{4}^{-} .
\end{aligned}
$$


Let $z \in \mathbf{f}$. Then since the inner product is nondegenerate, this equality is equivalent to the equality

$$
\begin{aligned}
\sum_{b_{3}, b_{4} \in B_{\nu}} c_{b_{3}, b_{4}} m_{i}^{p\left(b_{3}\right)}\left(b_{3}^{*}, z\right)\left(E_{i} b_{4}^{-}-\pi_{i}^{p\left(b_{3}\right)} b_{4}^{-} E_{i}\right) & \\
& +\sum_{b_{1}, b_{2} \in B_{\nu-i}} c_{b_{1}, b_{2}}\left(\left(\theta_{i} b_{1}^{*}, z\right) \tilde{K}_{-i} b_{2}^{-}-\pi_{i}^{p\left(b_{2}\right)}\left(b_{1}^{*} \theta_{i}, z\right) b_{2}^{-} \tilde{J}_{i} \tilde{K}_{i}\right)=0
\end{aligned}
$$

and

$$
\begin{aligned}
\sum_{b_{3}, b_{4} \in B_{\nu}} c_{b_{3}, b_{4}} \pi_{i}^{p\left(b_{4}\right)}\left(b_{4}, z\right)\left(b_{3}^{*+} F_{i}-\pi_{i}^{p\left(b_{4}\right)} b_{3}^{*+} F_{i}\right) \\
\quad+\sum_{b_{1}, b_{2} \in B_{\nu-i}} c_{b_{1}, b_{2}}\left(\left(b_{2} \theta_{i}, z\right) b_{1}^{*+} \tilde{K}_{-i}-\pi_{i}^{p\left(b_{1}\right)}\left(\theta_{i} b_{2}, z\right) \tilde{J}_{i} \tilde{K}_{i} b_{1}^{*+}\right)=0 .
\end{aligned}
$$

Note that $p\left(b_{1}\right)+p(i)=p\left(b_{2}\right)+p(i)=p\left(b_{3}\right)=p\left(b_{4}\right)$. Using Proposition 3.6 and the derivations, we have

$$
\begin{gathered}
\sum_{b_{3}, b_{4} \in B_{\nu}}\left(\pi_{i} q_{i}-q_{i}^{-1}\right)^{-1} c_{b_{3}, b_{4}} \pi_{i}^{p\left(b_{3}\right)}\left(b_{3}^{*}, z\right)\left(\pi_{i}^{p\left(b_{4}\right)-p(i)} \bar{r}_{i}\left(b_{4}\right)^{-} \tilde{J}_{i} \tilde{K}_{i}-\tilde{K}_{-i} \bar{r}\left(b_{4}\right)^{-}\right) \\
+\sum_{b_{1}, b_{2} \in B_{\nu-i}} c_{b_{1}, b_{2}}\left(\theta_{i}, \theta_{i}\right)\left(\left(b_{1}^{*}, i r(z)\right) \tilde{K}_{-i} b_{2}^{-}-\pi_{i}^{p\left(b_{2}\right)}\left(b_{1}^{*}, r_{i}(z)\right) b_{2}^{-} \tilde{J}_{i} \tilde{K}_{i}\right)=0,
\end{gathered}
$$

and

$$
\begin{gathered}
\sum_{b_{3}, b_{4} \in B_{\nu}}\left(\pi_{i} q_{i}-q_{i}^{-1}\right)^{-1} c_{b_{3}, b_{4}} \pi_{i}^{p\left(b_{4}\right)}\left(b_{4}, z\right)\left(\pi_{i}^{p\left(b_{3}\right)-p(i)} \tilde{J}_{i} \tilde{K}_{i} \bar{r}\left(b_{3}^{*}\right)^{+}-\overline{r_{i}}\left(b_{3}^{*}\right)^{+} \tilde{K}_{-i}\right) \\
+\sum_{b_{1}, b_{2} \in B_{\nu-i}} c_{b_{1}, b_{2}}\left(\theta_{i}, \theta_{i}\right)\left(\left(b_{2}, r_{i}(z)\right) b_{1}^{*+} \tilde{K}_{-i}-\pi_{i}^{p\left(b_{1}\right)}\left(b_{2},{ }_{i} r(z)\right) \tilde{J}_{i} \tilde{K}_{i} b_{1}^{*+}\right)=0 .
\end{gathered}
$$

Using the triangular decomposition, this is equivalent to the equalities (in $\mathbf{f}$ )

$$
\begin{aligned}
& \sum_{b_{1}, b_{2}} c_{b_{1}, b_{2}}\left(b_{1}^{*}, r_{i}(z)\right) b_{2}+\sum_{b_{3}, b_{4}} q_{i} \pi_{i}^{p(\nu)} c_{b_{3}, b_{4}}\left(b_{3}^{*}, z\right) \overline{r_{i}}\left(b_{4}\right)=0, \\
& \sum_{b_{1}, b_{2}} c_{b_{1}, b_{2}}\left(b_{1}^{*},{ }_{i} r(z)\right) b_{2}+\sum_{b_{3}, b_{4}} q_{i} \pi_{i}^{p(\nu)} c_{b_{3}, b_{4}}\left(b_{3}^{*}, z\right) \overline{{ }_{i}} \bar{r}\left(b_{4}\right)=0, \\
& \sum_{b_{1}, b_{2}} c_{b_{1}, b_{2}}\left(b_{2},{ }_{i} r(z)\right) b_{1}^{*}+\sum_{b_{3}, b_{4}} q_{i} \pi_{i}^{p(\nu)} c_{b_{3}, b_{4}}\left(b_{4}, z\right) \bar{i} \bar{r}\left(b_{3}^{*}\right)=0, \\
& \sum_{b_{1}, b_{2}} c_{b_{1}, b_{2}}\left(b_{2}, r_{i}(z)\right) b_{1}^{*}+\sum_{b_{3}, b_{4}} q_{i} \pi_{i}^{p(\nu)} c_{b_{3}, b_{4}}\left(b_{4}, z\right) \overline{r_{i}}\left(b_{3}^{*}\right)=0 .
\end{aligned}
$$


Now suppose $c_{b, b^{\prime}}=(-1)^{\mathrm{ht}(\nu)} \pi^{e(\nu)} \pi_{\nu} q_{-\nu} \delta_{b, b^{\prime}}$. Note that $e(\nu)=p(\nu-i) p(i)+e(\nu-i)$. Then we have

$$
\begin{aligned}
& \sum_{b}\left(b^{*}, r_{i}(z)\right) b-\sum_{b^{\prime}}\left(b^{\prime *}, z\right) \overline{r_{i}}\left(b^{\prime}\right)=0 \\
& \sum_{b}\left(b^{*},{ }_{i} r(z)\right) b-\sum_{b^{\prime}}\left(b^{\prime *}, z\right) \overline{{ }_{i} r}\left(b^{\prime}\right)=0 \\
& \sum_{b}\left(b,{ }_{i} r(z)\right) b^{*}-\sum_{b^{\prime}}\left(b^{\prime}, z\right) \bar{i} \bar{r}\left(b^{\prime}\right)=0 \\
& \sum_{b}\left(b, r_{i}(z)\right) b^{*}-\sum_{b^{\prime}}\left(b^{\prime}, z\right) \overline{r_{i}}\left(b^{\prime}\right)=0 .
\end{aligned}
$$

These equalities are easily verified by checking when $z$ is a basis or dual basis element.

Thus the existence of such a $\Theta$ is verified. Suppose $\Theta_{\nu}^{\prime}$ and $\Theta^{\prime}$ also satisfy the conditions in (a). Then $\Theta-\Theta^{\prime}=\sum c_{b, b^{\prime}} b^{-} \otimes b^{\prime+}$ must satisfy (3.20)-(3.23) and has $c_{b, b}=0$ for $b \in B_{0}$. Suppose $c_{b, b^{\prime}}=0$ for $b, b^{\prime} \in B_{\nu}^{\prime}$ for $\operatorname{ht}\left(\nu^{\prime}\right)<n$ and assume $\operatorname{ht}(\nu)=n$. Then the first sum in $(3.20)$ is zero, so $\overline{r_{i}}\left(\sum_{b_{3}, b_{4}} c_{b_{3}, b_{4}}\left(b_{3}^{*}, z\right) b_{4}\right)=0$. But then $\sum_{b_{3}, b_{4}} c_{b_{3}, b_{4}}\left(b_{3}^{*}, z\right) b_{4}=0$, whence $\left(\sum_{b_{3}, b_{4}} c_{b_{3}, b_{4}} b_{3}^{*}, z\right)=0$ for all $z \in \mathbf{f}$. Therefore $c_{b_{3}, b_{4}}=0$ for all $b_{1}, b_{2} \in B_{\nu}$. By induction $\Theta-\Theta^{\prime}=0$ proving uniqueness.

Recall that the bar involution on $\mathbf{U}$ makes sense under the assumption that the super Cartan datum is consistent.

Corollary 3.34. Assume that the super Cartan datum is consistent. We have $\Theta \bar{\Theta}=$ $\bar{\Theta} \Theta=1 \otimes 1$ with equality in the completion.

Proof. First note that by construction $\Theta$ is invertible. We have $\Delta(u) \Theta=\Theta \bar{\Delta}(u)$, so $\Theta \Delta(\bar{u})=\Theta \bar{\Delta}(\bar{u})=\Theta \overline{\Delta(u)}$. Now applying the bar involution to both sides and rearranging, we get

$$
\bar{\Theta}^{-1} \bar{\Delta}(u)=\Delta(u) \bar{\Theta}^{-1}
$$

By uniqueness, $\bar{\Theta}^{-1}=\Theta$. 
We can specialize the identity $\Delta(u) \Theta=\Theta \bar{\Delta}(u)$ to deduce

$$
\begin{aligned}
& \left(E_{i} \otimes \tilde{K}_{-i}\right) \Theta_{\nu-i}+\left(1 \otimes E_{i}\right) \Theta_{\nu}=\Theta_{\nu-i}\left(E_{i} \otimes \tilde{J}_{i} \tilde{K}_{i}\right)+\Theta_{\nu}\left(1 \otimes E_{i}\right), \\
& \left(\tilde{J}_{i} \tilde{K}_{i} \otimes F_{i}\right) \Theta_{\nu-i}+\left(F_{i} \otimes 1\right) \Theta_{\nu}=\Theta_{\nu-i}\left(\tilde{K}_{-i} \otimes F_{i}\right)+\Theta_{\nu}\left(F_{i} \otimes 1\right) .
\end{aligned}
$$

Setting $\Theta_{\leq p}=\sum_{\mathrm{ht} \nu \leq p} \Theta_{\nu}$, we obtain that

$$
\begin{aligned}
\left(E_{i} \otimes \tilde{K}_{-i}+1 \otimes E_{i}\right) \Theta_{\leq p}-\Theta_{\leq p}\left(E_{i} \otimes \tilde{J}_{i} \tilde{K}_{i}+1 \otimes E_{i}\right) & \\
= & \sum_{\mathrm{h} t \nu=p}\left(E_{i} \otimes \tilde{K}_{-i}\right) \Theta_{\nu}-\sum_{\mathrm{h} t \nu=p} \Theta_{\nu}\left(E_{i} \otimes \tilde{J}_{i} \tilde{K}_{i}\right), \\
\left(F_{i} \otimes 1+\tilde{J}_{i} \tilde{K}_{i} \otimes F_{i}\right) & \Theta_{\leq p}-\Theta_{\leq p}\left(F_{i} \otimes 1+\tilde{K}_{-i} \otimes F_{i}\right) \\
= & \sum_{\mathrm{h} t \nu=p}\left(\tilde{J}_{i} \tilde{K}_{i} \otimes F_{i}\right) \Theta_{\nu}-\sum_{\mathrm{h} t \nu=p} \Theta_{\nu}\left(\tilde{K}_{-i} \otimes F_{i}\right) .
\end{aligned}
$$

There is a variant of the quasi- $\mathcal{R}$-matrix for the coproduct $\Delta^{\prime}$. Let $\bar{\Delta}: \mathbf{U} \rightarrow \mathbf{U} \otimes \mathbf{U}$ be the $\mathbb{Q}(q)^{\pi}$-algebra homomorphism given by $\bar{\Delta}(x)=\overline{\Delta(\bar{x})}$.

Corollary 3.35. Let $\Theta^{\prime}=\sum_{\nu}\left(\tilde{J}_{\nu} \otimes 1\right) \Theta_{\nu}$. Then $\Delta^{\prime}(u) \Theta=\Theta \overline{\Delta^{\prime}}(u)$ for all $u \in \mathbf{U}$ (where this identity is in $\left.(\mathbf{U} \otimes \mathbf{U})^{\wedge}\right)$.

Proof. Note that $\left(\tilde{J}_{\nu} \otimes 1\right)$ is central in $\mathbf{U} \otimes \mathbf{U}$. We have

$$
\left(1 \otimes E_{i}\right) \Theta_{\nu}-\Theta_{\nu}\left(1 \otimes E_{i}\right)=\Theta_{\nu-i}\left(E_{i} \otimes \tilde{J}_{i} \tilde{K}_{i}\right)-\left(E_{i} \otimes \tilde{K}_{-i}\right) \Theta_{\nu-i},
$$

in $\mathbf{U} \otimes \mathbf{U}$, which implies

$$
\left(\tilde{J}_{i} \otimes E_{i}\right) \Theta_{\nu}-\Theta_{\nu}\left(\tilde{J}_{i} \otimes E_{i}\right)=\left(\tilde{J}_{i} \otimes 1\right) \Theta_{\nu-i}\left(E_{i} \otimes \tilde{J}_{i} \tilde{K}_{i}\right)-\left(E_{i} \otimes \tilde{K}_{-i}\right)\left(\tilde{J}_{i} \otimes 1\right) \Theta_{\nu-i} .
$$

Multiplying both sides by $\tilde{J}_{\nu}$,

$$
\left(\tilde{J}_{i} \otimes E_{i}\right) \Theta_{\nu}^{\prime}-\Theta_{\nu}^{\prime}\left(\tilde{J}_{i} \otimes E_{i}\right)=\Theta_{\nu-i}^{\prime}\left(E_{i} \otimes \tilde{J}_{i} \tilde{K}_{i}\right)-\left(E_{i} \otimes \tilde{K}_{-i}\right) \Theta_{\nu-i}^{\prime} .
$$

A similar argument applies to $F$, so we have

$$
\begin{aligned}
\left(E_{i} \otimes \tilde{K}_{-i}\right) \Theta_{\nu-i}^{\prime}+\left(\tilde{J}_{i} \otimes E_{i}\right) \Theta_{\nu}^{\prime} & =\Theta_{\nu-i}^{\prime}\left(E_{i} \otimes \tilde{J}_{i} \tilde{K}_{i}\right)+\Theta_{\nu}^{\prime}\left(\tilde{J}_{i} \otimes E_{i}\right), \\
\left(\tilde{K}_{i} \otimes F_{i}\right) \Theta_{\nu-i}^{\prime}+\left(F_{i} \otimes 1\right) \Theta_{\nu}^{\prime} & =\Theta_{\nu-i}^{\prime}\left(\tilde{J}_{i} \tilde{K}_{-i} \otimes F_{i}\right)+\Theta_{\nu}^{\prime}\left(F_{i} \otimes 1\right) .
\end{aligned}
$$

Then we have analogues of (3.24), (3.25) and so by continuity, we obtain the desired equality. 


\subsection{The quantum Casimir}

Let $B, B_{\nu}$ be as in Theorem 3.33. Let $S$ be the antipode and $\mathbf{m}: \mathbf{U} \otimes \mathbf{U} \rightarrow \mathbf{U}$ be the multiplication map $u \otimes u^{\prime} \mapsto u u^{\prime}$. Applying $\mathbf{m}(S \otimes 1)$ to the identities (3.24) and (3.25), we obtain that, for any $p \geq 0$,

$$
\begin{gathered}
\sum_{\text {ht } \nu \leq p} \sum_{b \in B_{\nu}}(-1)^{\mathrm{ht} \nu} \pi_{\nu} q_{-\nu}\left(S\left(E_{i} b^{*+}\right) \tilde{K}_{-i} b^{-}+\pi_{i}^{p(\nu)} S\left(b^{*+}\right) E_{i} b^{-}\right. \\
\left.-\pi_{i}^{p(\nu)} S\left(b^{*+} E_{i}\right) b^{-} \tilde{J}_{i} \tilde{K}_{i}-S\left(b^{*+}\right) b^{-} E_{i}\right) \\
=\sum_{\text {ht } \nu=p} \sum_{b \in B_{\nu}}(-1)^{p} \pi_{\nu} q_{-\nu}\left(S\left(E_{i} b^{*+}\right) \tilde{K}_{-i} b^{-}-\pi_{i}^{p(\nu)} S\left(b^{*+} E_{i}\right) b^{-} \tilde{J}_{i} \tilde{K}_{i}\right),
\end{gathered}
$$

and

$$
\begin{gathered}
\sum_{\mathrm{ht} \nu \leq p} \sum_{b \in B_{\nu}}(-1)^{\mathrm{ht} \nu} \pi_{\nu} q_{-\nu}\left(S\left(F_{i} b^{*+}\right) b^{-}+\pi_{i}^{p(\nu)} S\left(\tilde{J}_{i} \tilde{K}_{i} b^{*+}\right) F_{i} b^{-}\right. \\
\left.-\pi_{i}^{p(\nu)} S\left(b^{*+} F_{i}\right) b^{-}-S\left(b^{*+} \tilde{K}_{-i}\right) b^{-} F_{i}\right) \\
=\sum_{\text {ht } \nu=p} \sum_{b \in B_{\nu}}(-1)^{p} \pi_{\nu} q_{-\nu}\left(\pi_{i}^{p(\nu)} S\left(\tilde{J}_{i} \tilde{K}_{i} b^{*+}\right) F_{i} b^{-}-S\left(b^{*+} \tilde{K}_{-i}\right) b^{-} F_{i}\right) .
\end{gathered}
$$

Now set $\Omega_{\leq p}^{\prime}=\sum_{\mathrm{ht} \nu \leq p} \sum_{b \in B_{\nu}}(-1)^{\mathrm{ht} \nu} \pi_{\nu} q_{-\nu} S\left(b^{*+}\right) b^{-}$. Then observing that

$$
\begin{aligned}
S\left(E_{i} b^{*+}\right) \tilde{K}_{-i} b^{-} & +\pi_{i}^{p(\nu)} S\left(b^{*+}\right) E_{i} b^{-} \\
& =-\pi_{i}^{p(\nu)} S\left(b^{*+}\right) E_{i} b^{-}+\pi_{i}^{p(\nu)} S\left(b^{*+}\right) E_{i} b^{-}=0,
\end{aligned}
$$

and that a similar identity for $F_{i}$ exists, we have

$$
\begin{aligned}
& E_{i} \tilde{K}_{i} \Omega_{\leq p}^{\prime} \tilde{J}_{i} \tilde{K}_{i}-\Omega_{\leq p}^{\prime} E_{i} \\
&= \sum_{\text {ht } \nu=p} \sum_{b \in B_{\nu}}(-1)^{p} \pi_{\nu} q_{-\nu}\left(\pi_{i}^{p(\nu)} S\left(E_{i} b^{*+}\right) \tilde{K}_{-i} b^{-}-S\left(b^{*+} E_{i}\right) \tilde{K}_{i} \tilde{J}_{i} b^{-}\right), \\
& \tilde{J}_{-i} \tilde{K}_{-i} F_{i} \Omega_{\leq p}^{\prime}-\tilde{K}_{i} \Omega_{\leq p}^{\prime} F_{i} \\
&=\left(\pi_{i}^{p(\nu)} S\left(\tilde{J}_{i} \tilde{K}_{i} b^{*+}\right) F_{i} b^{-}-S\left(b^{*+} \tilde{K}_{-i}\right) b^{-} F_{i}\right) .
\end{aligned}
$$

Now we set $\Omega_{\leq p}=S^{\prime}\left(\Omega_{\leq p}^{\prime}\right)$. Let $M \in \mathcal{O}$. Then for any $m \in M$ we have that $\Omega(m)=\Omega_{\leq p} m$ is independent of $p$ when $p$ is large enough. We can write

$$
\Omega(m)=\sum_{b}(-1)^{\mathrm{ht}|b|} q_{-|b|} S^{\prime}\left(b^{-}\right) b^{*+} m
$$


We then have

$$
\tilde{K}_{i} E_{i} \Omega_{\leq p}^{\prime}=\tilde{J}_{-i} \tilde{K}_{-i} \Omega_{\leq p}^{\prime} E_{i}, \quad \Omega F_{i}=F_{i} \tilde{J}_{-i} \tilde{K}_{-i} \Omega \tilde{K}_{-i}, \quad \Omega J_{\nu} K_{\mu}=J_{\nu} K_{\mu} \Omega,
$$

as operators on $M$. Therefore for $m \in M_{\lambda}$, we have

$$
\Omega E_{i} m=\left(\pi_{i} q_{i}^{2}\right)^{\left\langle i, \lambda+i^{\prime}\right\rangle} E_{i} \Omega m, \quad \Omega F_{i} m=\left(\pi_{i} q_{i}^{2}\right)^{-\langle i, \lambda\rangle} F_{i} \Omega m
$$

Let $C$ be a fixed coset of $X$ with respect to $\mathbb{Z}[I] \leq X$. Let $G: C \rightarrow \mathbb{Z}$ be a function such that

$$
G(\lambda)-G\left(\lambda-i^{\prime}\right)=\frac{i \cdot i}{2}\langle i, \lambda\rangle \quad \text { for all } \lambda \in C, i \in I
$$

Clearly such a function exists and is unique up to addition of a constant function.

Lemma 3.36. Let $\lambda, \lambda^{\prime} \in C \cap X^{+}$. If $\lambda \geq \lambda^{\prime}$ and $G(\lambda)=G\left(\lambda^{\prime}\right)$, then $\lambda=\lambda^{\prime}$.

Let $M \in \mathcal{C}$. For each $\mathbb{Z}[I]$-coset $C$ in $X$, define $M_{C}=\bigoplus_{\lambda \in C} M_{\lambda}$. It is clear that

$$
M=\bigoplus_{C \in X / \mathbb{Z}[I]} M_{C}
$$

Proposition 3.37. Let $M \in \mathcal{O}$, and let $\Omega: M \rightarrow M$ be as above.

(a) Assume there exists $C$ as above such that $M=M_{C}$. Let $G: C \rightarrow \mathbb{Z}$ be a function satisfying (3.26). We define a linear map $\Xi: M \rightarrow M$ by $\Xi(m)=$ $\left(\pi q^{2}\right)^{-G(\lambda)} m$ for all $\lambda \in C$ and $m \in M_{\lambda}$. Then $\Omega \Xi$ is a locally finite $U$-module homomorphism.

(b) Assume that $M$ is a quotient of $M\left(\lambda^{\prime}\right)$. Then $\Omega \Xi$ acts as $\left(\pi q^{2}\right)^{-G\left(\lambda^{\prime}\right)}$ on $M$.

(c) For $M$ as in (a), the eigenvalues of $\Omega \Xi$ are of the form $\left(\pi q^{2}\right)^{c}$ for $c \in \mathbb{Z}$.

The operator $\Omega \Xi$ is called the Casimir element of $\mathbf{U}$ (though note that the Casimir element formally lives in a completion of $\mathbf{U}$ ). 
Proof. We compute that for $m \in M_{\lambda}$,

$$
\Omega \Xi E_{i} m=\Omega\left(\pi q^{2}\right)^{-G\left(\lambda+i^{\prime}\right)} E_{i} m=\left(\pi q^{2}\right)^{-G\left(\lambda+i^{\prime}\right)+G(\lambda)+d_{i}\left\langle i, \lambda+i^{\prime}\right\rangle} E_{i} \Omega \Xi m=E_{i} \Omega \Xi m .
$$

A similar argument applies to the $F_{i}$, and clearly $\Omega \Xi$ commutes with $K_{\mu}, J_{\mu}$ proving the first assertion of (a). The local finiteness claim is a standard category $\mathcal{O}$ type argument. Parts (b) and (c) follow now easily.

\subsection{Complete reducibility in $\mathcal{O}_{\text {int }}$}

Recall the categories $\mathcal{O}$ and $\mathcal{C}_{\text {int }}$, and form another category $\mathcal{O}_{\text {int }}:=\mathcal{O} \cap \mathcal{C}_{\text {int }}$.

Lemma 3.38. Let $M \in \mathcal{C}$. Assume that $M$ is a nonzero quotient of the Verma module $M(\lambda)$ and that $M$ is integrable. Then

(a) $\lambda \in X^{+}$;

(b) $\left.M\right|_{\pi=1}$ and $\left.M\right|_{\pi=-1}$ are either simple or zero.

Proof. It is clear that (a) holds by some rank one consideration. An argument similar to that for [Lu4, Lemma 6.2.1] shows that if $\operatorname{dim}_{\mathbb{Q}(q)} M_{\lambda}=1$ then $M$ is simple; in this case, $M$ must be equal to either $\left.M\right|_{\pi=1}$ or $\left.M\right|_{\pi=-1}$. Otherwise, $\operatorname{dim}_{\mathbb{Q}(q)} M_{\lambda}=2$, then $\left.\operatorname{dim}_{\mathbb{Q}(q)} M_{\lambda}\right|_{\pi=1}=\left.\operatorname{dim}_{\mathbb{Q}(q)} M_{\lambda}\right|_{\pi=-1}=1$, and we repeat the argument above for the integrable $\mathbf{U}$-module $\left.M\right|_{\pi= \pm 1}$.

Theorem 3.39. Let $M$ be a $\mathbf{U}$-module in $\mathcal{O}_{\text {int }}$. Then $M$ is a sum of simple $\mathbf{U}$ submodules.

Proof. Note that as discussed in $\S 3.5$ we may assume that $M=\left.M\right|_{\pi=1}$ or $M=$ $\left.M\right|_{\pi=-1}$. Since the case for $\left.M\right|_{\pi=1}$ follows from [Lu4, Theorem 6.2.2], it is enough to prove the theorem for $M=\left.M\right|_{\pi=-1}$. Virtually the same argument as in loc. cit. holds, which we will now sketch. 
Using (3.27), we may further assume there is a coset $C$ of $\mathbb{Z}[I]$ in $X$ such that $M=M_{C}$. Then we may pick a function $G$ satisfying (3.26) and avail ourselves of Proposition 3.37. Since the Casimir element commutes with the U-action, we may further assume that $M$ lies in a generalized eigenspace of the Casimir element.

Consider the set of singular vectors of $M$ (that is, the set of vectors $m \in M$ for which $E_{i} m=0$ for all $\left.i \in I\right)$ and let $M^{\prime}$ be the submodule they generate. Then each homogeneous singular vector generates a simple submodule by virtue of Lemma 3.38, so $M^{\prime}$ is a sum of simple modules.

It remains to show that $M=M^{\prime}$, so take $M^{\prime \prime}=M / M^{\prime}$ and suppose $M^{\prime \prime} \neq 0$. Then there is a maximal weight $\lambda \in C$ such that $M_{\lambda}^{\prime \prime} \neq 0$. Then the Casimir element acts on the submodule generated by a nonzero $m_{1} \in M_{\lambda}^{\prime \prime}$ by $\left(-q^{2}\right)^{-G(\lambda)}$ by Proposition 3.37, and so in particular $M$ must lie in the generalized $\left(-q^{2}\right)^{-G(\lambda)}$-eigenspace of the Casimir element.

On the other hand, $m$ is the image of a vector $\tilde{m} \in M \backslash M^{\prime}$. The $\mathbf{U}^{+}$-module generated by $\tilde{m}$ contains a singular vector $m_{2}$ of weight $\eta \geq \lambda$, and the Casimir element acts on the module generated by $m_{2}$ as $\left(-q^{2}\right)^{-G(\eta)}$. Then $G(\eta)=G(\lambda)$ and $\eta \geq \lambda$, so by Lemma $3.36 \eta=\lambda$. But the $\tilde{m}$ is a singular vector, contradicting that our choice of $m_{1}$ was nonzero.

Corollary 3.40. (a) For $\lambda \in X^{+}$, the $\mathbf{U}$-modules $\left.V(\lambda)\right|_{\pi=1}$ and $\left.V(\lambda)\right|_{\pi=-1}$ are simple objects of $\mathcal{O}_{\text {int }}$.

(b) For $\lambda, \lambda^{\prime} \in X^{+}$, the $\mathbf{U}$-modules $\left.V(\lambda)\right|_{\pi=1}$ and $\left.V\left(\lambda^{\prime}\right)\right|_{\pi=1}$, and respectively the modules $\left.V(\lambda)\right|_{\pi=-1}$ and $\left.V\left(\lambda^{\prime}\right)\right|_{\pi=-1}$, are isomorphic if and only if $\lambda=\lambda^{\prime}$. (Clearly, $\left.V(\lambda)\right|_{\pi=1}$ and $\left.V\left(\lambda^{\prime}\right)\right|_{\pi=-1}$ are non-isomorphic.)

(c) Any integrable module in $\mathcal{O}$ is a direct sum of simple modules of the form $\left.V(\lambda)\right|_{\pi= \pm 1}$ for various $\lambda \in X^{+}$.

Proof. The argument in [Lu4, Corollary 6.2.3] holds using our Lemma 3.38 above. 
In light of Corollary 3.40 and Remark 3.29, we are lead to the following definition. We say a $\mathbf{U}$-module $M$ is $\pi$-free if it is a weight module such that $M_{\lambda}$ is a free $\mathbb{Q}(q)^{\pi}$ module for all $\lambda \in X$. Then the category of $\pi$-free modules contains most of the interesting modules; for example, the modules $M(\lambda), V(\lambda), N\left(\lambda, \lambda^{\prime}\right)$, and indeed $\mathbf{U}$ itself all lie in this category. In fact, each module in $\mathcal{O}_{\text {int }}$ is a direct summand of a $\pi$-free module.

One reason to assume modules are $\pi$-free is to utilize the following definition.

Definition 3.41. Let $R$ be a ring. A $\pi$-basis for a free $R^{\pi}$-module $M$ is a set $S \subset M$ such that there exists an $R^{\pi}$-basis $B$ for $M$ with $S=B \cup \pi B$.

Suppose $M$ and $M^{\prime}$ are free $R^{\pi}$-modules with $\pi$-bases $S \subset M$ and $S^{\prime} \subset M^{\prime}$. The $S \otimes S=\left\{s \otimes s \mid s \in S, s^{\prime} \in S^{\prime}\right\}$ is clearly a $\pi$-basis for $M \otimes M^{\prime}$. However, we note the map $S \times S^{\prime} \rightarrow S \otimes S^{\prime}$ given by $\left(s, s^{\prime}\right) \mapsto s \otimes s^{\prime}$ is not a bijection, since clearly $s \otimes s^{\prime}=(\pi s) \otimes\left(\pi s^{\prime}\right)$. We will occasionally need an honest index set, so let $\sim$ be the equivalence relation on $S \times S^{\prime}$ given by $\left(\pi s, s^{\prime}\right) \sim\left(s, \pi s^{\prime}\right)$. Then we set

$$
S \times_{\pi} S^{\prime}=S \times S^{\prime} / \sim
$$

We note that a $\pi$-basis of an $R^{\pi}$-module $M$ is an $R$-basis of $M$. We will now turn to constructing a particular $\pi$-basis for $\mathbf{U}^{-}$and the modules $V(\lambda)$. 


\section{Chapter 4}

\section{Crystal basis and the grand loop}

\subsection{The $(q, \pi)$-boson superalgebra}

From now on, we always assume that the root datum is $Y$-regular. Let $\mathbf{F}=\mathbf{F}_{q, \pi}$ be the $\mathbb{Q}(q)^{\pi}$-superalgebra generated by odd elements $e, f$ subject to the relation

$$
e f=\pi q^{-2} f e+1
$$

We set $f^{(n)}=f^{n} /[n]^{!}$.

One checks that

$$
e^{n} f^{(m)}=\sum_{t \geq 0}(\pi q)^{\left(\begin{array}{c}
t+1 \\
2
\end{array}\right)-n m} q^{-(n-t)(m-t)}\left[\begin{array}{l}
n \\
t
\end{array}\right]_{q, \pi} f^{(m-t)} e^{n-t} .
$$

The following properties may be directly verified.

Lemma 4.1. Let $M$ be a $\mathbf{F}$-module which is locally finite for e.

(i) $P=\sum_{n \geq 0}(-1)^{n} q^{-\left(\begin{array}{c}n \\ 2\end{array}\right)} f^{(n)} e^{n}$ defines an endomorphism of $M$ satisfying

$$
e P=P f=0 \text { and } \sum_{t \geq 0}(\pi q)^{\left(\begin{array}{c}
t \\
2
\end{array}\right)} f^{(t)} P e^{t}=1 .
$$

(ii) Let $m \in M$. Then any $u \in M$ has a unique decomposition $u=\sum_{n \geq 0} f^{(n)} u_{n}$ where $u_{n} \in$ ker $e$; in fact, $u_{n}=(\pi q)^{\left(\begin{array}{l}n \\ 2\end{array}\right)} P e^{n} u$.

(iii) $M=\operatorname{im} f \oplus \operatorname{ker} e$. Moreover $P: M \rightarrow M$ is the projection map onto ker $e$ along this direct sum decomposition. 
Define the algebra $\mathbf{B}^{\prime}$ to be the $\mathbb{Q}(q)^{\pi}$-algebra generated by the elements $e_{i}$ and $f_{i}$ for $i \in I$, subject to the relations

$$
e_{i} f_{j}=\pi^{p(i) p(j)} q_{i}^{-a_{i j}} f_{j} e_{i}+\delta_{i j}, \quad \text { for all } i, j \in I
$$

Then $\mathbf{B}^{\prime}$ is naturally a superalgebra with parity on generators given by $p\left(e_{i}\right)=p\left(f_{i}\right)=$ $p(i)$ for $i \in I$. Set $f_{i}^{(n)}=f_{i}^{n} /[n]_{i}^{!}$. The superalgebra $\mathbf{B}$ by definition has the same generators as $\mathbf{B}^{\prime}$ subject to the relation (4.3) and the additional $(q, \pi)$-Serre relations $(4.4)$ :

$$
\begin{aligned}
& \sum_{t=0}^{b_{i j}}(-1)^{t} \pi_{i}^{\left(\begin{array}{c}
t \\
2
\end{array}\right)+t p(j)}\left[\begin{array}{c}
b_{i j} \\
t
\end{array}\right]_{q_{i}, \pi_{i}} e_{i}^{b_{i j}-t} e_{j} e_{i}^{t}=0 \\
& \sum_{t=0}^{b_{i j}}(-1)^{t} \pi_{i}^{\left(\begin{array}{c}
t \\
2
\end{array}\right)+t p(j)}\left[\begin{array}{c}
b_{i j} \\
t
\end{array}\right]_{q_{i}, \pi_{i}} f_{i}^{b_{i j}-t} f_{j} f_{i}^{t}=0
\end{aligned}
$$

where we recall that

$$
b_{i j}=1-a_{i j}
$$

Let

$$
s_{i j}=\sum_{t=0}^{b_{i j}}(-1)^{t} \pi_{i}^{\left(\begin{array}{l}
t \\
2
\end{array}\right)+t p(j)}\left[\begin{array}{c}
b_{i j} \\
t
\end{array}\right]_{q_{i}, \pi_{i}} e_{i}^{b_{i j}-t} e_{j} e_{i}^{t} \in \mathbf{B}^{\prime} .
$$

Lemma 4.2. The following holds in $\mathbf{B}^{\prime}$ for all $i, j, k \in I$ with $i \neq j$ :

$$
s_{i j} f_{k}=\pi_{k}^{b_{i j} p(i)+p(j)} q_{k}^{-\left\langle k, b_{i j} i^{\prime}+j^{\prime}\right\rangle} f_{k} s_{i j} .
$$

Proof. Let $C_{i j}^{k}=s_{i j} f_{k}-\pi_{k}^{b_{i j} p(i)+p(j)} q_{k}^{-\left\langle k, b_{i j} i^{\prime}+j^{\prime}\right\rangle} f_{k} S_{i j}$.

If $k \neq i, j$ then then it is apparent that $C_{i j}^{k}=0$ from the defining relations.

When $k=j$, then we have

$$
C_{i j}^{j}=e_{i}^{b_{i j}} \sum_{t=0}^{b_{i j}}(-1)^{t} q_{i}^{-t a_{i j}} \pi_{i}^{\left(\begin{array}{l}
t \\
2
\end{array}\right)}\left[\begin{array}{c}
b_{i j} \\
t
\end{array}\right]_{q_{i}, \pi_{i}}=0
$$

by using $1-a_{i j}=b_{i j}$ and the identity $\sum_{t=0}^{n}(-1)^{t} q_{i}^{t(n-1)} \pi_{i}^{\left(\begin{array}{l}t \\ 2\end{array}\right)}\left[\begin{array}{c}n \\ t\end{array}\right]_{q_{i}, \pi_{i}}=0$.

Finally, if $k=i$ then we have 


$$
\begin{aligned}
C_{i j}^{i}=\sum_{t=0}^{b_{i j}-1}( & -1)^{t} q_{i}^{-t} e_{i}^{b_{i j}-t-1} e_{j} e_{i}^{t} \pi_{i}^{\left(\begin{array}{l}
t \\
2
\end{array}\right)+(t+1) p(j)} \\
& \times\left(\pi_{i}^{a_{i j}}\left[\begin{array}{c}
b_{i j} \\
t
\end{array}\right]_{q_{i}, \pi_{i}}\left[b_{i j}-t\right]_{q_{i}, \pi_{i}}-\left[\begin{array}{c}
b_{i j} \\
t+1
\end{array}\right]_{q_{i}, \pi_{i}}[t+1]_{q_{i}, \pi_{i}}\right) .
\end{aligned}
$$

We see that $\pi_{i}^{a_{i j}}\left[\begin{array}{c}b_{i j} \\ t\end{array}\right]_{q_{i}, \pi_{i}}\left[b_{i j}-t\right]_{q_{i}, \pi_{i}}-\left[\begin{array}{c}b_{i j} \\ t+1\end{array}\right]_{q_{i}, \pi_{i}}[t+1]_{q_{i}, \pi_{i}}=0$ by noting that we have $\left[\begin{array}{c}n \\ t\end{array}\right]_{q_{i}, \pi_{i}}[n-t]_{q_{i}, \pi_{i}}=\left[\begin{array}{c}n \\ t+1\end{array}\right]_{q_{i}, \pi_{i}}[t+1]_{q_{i}, \pi_{i}}$ and $a_{i j} p(i) \in 2 \mathbb{Z}$. The lemma is proved.

Remark 4.3. A multi-parameter version of the quantum boson algebra can also be found in [KKO].

Comparing with Propositions 3.5 and 3.5 and using the triangular decomposition, we see that if $x \in \mathbf{f}$ and $x^{-}=y \in \mathbf{U}^{-}$

$$
\left[E_{i}, y\right]=\frac{\tilde{J}_{i} \tilde{K}_{i}{ }_{i} r(x)^{-}-\tilde{K}_{-i} \bar{i} r(x)^{-}}{\pi_{i} q_{i}-q_{i}{ }^{-1}}, \quad \text { for } y=x^{-} \in \mathbf{U}^{-} .
$$

In particular, we record the following formulae

Lemma 4.4. For $y \in \mathbf{f}$, we have

$$
\begin{aligned}
& \overline{{ }_{i} r}\left(\theta_{j} y\right)=\pi_{i}^{p(j)} q_{i}^{-\left\langle i, j^{\prime}\right\rangle} \theta_{j} \bar{i} r(y)+\delta_{i j} y \\
& { }_{i} r\left(\theta_{j} y\right)=\pi_{i}^{p(j)} q_{i}^{\left\langle i, j^{\prime}\right\rangle} \theta_{j i} r(y)+\delta_{i j} y .
\end{aligned}
$$

Proposition 4.5. We have $\bar{i}_{i} r=\pi^{p(i) p(j)} q_{j}^{\left\langle j, i^{\prime}\right\rangle}{ }_{j} r \bar{i}{ }_{i}$, for $i, j \in I$.

Proof. Let $\nu \in \mathbb{N}[I]$ and let $y \in \mathbf{f}_{\nu}$. If ht $\nu \leq 1$, then $\bar{i}_{i}{ }_{j} r(y)=0={ }_{j}{ }{ }_{i} r(y)$. Otherwise, we may assume $y=\theta_{k} y^{\prime}$ for some $k \in I$ and $y^{\prime} \in U^{-}$with $\operatorname{ht}\left(-\left|y^{\prime}\right|\right)<\operatorname{ht}(-\nu)$. Then

$$
\begin{aligned}
\bar{i}_{i}{ }_{j} r(y) & =\overline{{ }_{i} r}\left(\pi_{j}^{p(k)} q_{j}^{\left\langle j, k^{\prime}\right\rangle} \theta_{k j} r\left(y^{\prime}\right)+\delta_{j k} y^{\prime}\right) \\
& =f(i, j, k) \theta_{k} \bar{i}_{i}{ }_{j} r\left(y^{\prime}\right)+\pi_{j}^{p(k)} q_{j}^{\left\langle j, k^{\prime}\right\rangle} \delta_{i k j} r\left(y^{\prime}\right)+\delta_{j k} \overline{{ }_{i} r}\left(y^{\prime}\right) \\
& =f(i, j, k) \theta_{k} \bar{i}_{i}{ }_{j} r\left(y^{\prime}\right)+\pi^{p(i) p(j)} q^{d_{j}\left\langle j, i^{\prime}\right\rangle} \delta_{i k j} r\left(y^{\prime}\right)+\delta_{j k} \bar{i} r\left(y^{\prime}\right)
\end{aligned}
$$


and similarly

$$
{ }_{j} r \bar{i} \bar{r}(y)=f(i, j, k) \theta_{k j} r \bar{i} \bar{r}\left(y^{\prime}\right)+\pi^{p(i) p(j)} q^{-d_{i}\left\langle i, j^{\prime}\right\rangle} \delta_{j k} \bar{i}\left(y^{\prime}\right)+\delta_{i k j} r\left(y^{\prime}\right)
$$

where we have denoted $f(i, j, k)=\pi^{(p(i)+p(j)) p(k)} q_{j}^{\left\langle j, k^{\prime}\right\rangle} q^{-d_{i}\left\langle i, k^{\prime}\right\rangle+d_{j}\left\langle j, k^{\prime}\right\rangle}$.

Note that $d_{j}\left\langle j, i^{\prime}\right\rangle=d_{i}\left\langle i, j^{\prime}\right\rangle$ and by induction $\bar{i}_{i}{ }_{j} r\left(y^{\prime}\right)=\pi^{p(i) p(j)} q_{j}^{\left\langle j, i^{\prime}\right\rangle}{ }_{j} r \bar{i} \bar{r}\left(y^{\prime}\right)$. Therefore, we have $\bar{i}_{i}{ }_{j} r\left(y^{\prime}\right)(y)=\pi^{p(i) p(j)} q_{j}^{\left\langle j, i^{\prime}\right\rangle}{ }_{j} r \bar{i} r(y)$.

Lemma 4.6. Let $i \in I$ and $x \in \mathbf{f}_{\zeta}$ such that ${ }_{i}{ }^{r}(x)=0$. Then for any $\mathbf{U}$-module $M$ and $m \in M_{\lambda}$ such that $e_{i} m=0$, we have

$$
\tilde{K}_{i}^{n} E_{i}^{n} u m=\pi_{i}^{n\langle i, \lambda\rangle} \frac{q_{i}^{n\left(2\left\langle i, \lambda+\zeta^{\prime}\right\rangle+3 n+1\right)}}{\left(\pi_{i} q_{i}-q_{i}^{-1}\right)^{n}}\left({ }_{i} r^{n} x\right)^{-} m .
$$

Proof. This lemma has essentially the same proof as [Kas1, Lemma 3.4.6]. The power of $\pi$ comes from the central element $\tilde{J}_{i}$.

Our interest in the superalgebra $\mathbf{B}$ comes from the following result.

Proposition 4.7. $\mathbf{f}$ is a $\mathbf{B}^{\prime}$-module as well as a $\mathbf{B}$-module, where $f_{i}$ acts as multiplication by $\theta_{i}$ and $e_{i}$ acts by the map ${ }_{i^{r}}$ for all $i \in I$.

Proof. By Lemma 4.4, $\mathbf{f}$ is a $\mathbf{B}^{\prime}$-module. Recall the Serre elements $s_{i j} \in \mathbf{B}^{\prime}$ from (4.5) and denote the $f$-counterparts by

$$
s_{i j}^{\prime}=\sum_{t=0}^{b_{i j}}(-1)^{t} \pi_{i}^{\left(\begin{array}{l}
t \\
2
\end{array}\right)+t p(j)}\left[\begin{array}{c}
b_{i j} \\
t
\end{array}\right] f_{i}^{b_{i j}-t} f_{j} f_{i}^{t} \in \mathbf{B}^{\prime} .
$$

To show that $\mathbf{f}$ is a $\mathbf{B}$-module, it suffices to show that $s_{i j}$ and $s_{i j}^{\prime}$ act as zero on any $y \in \mathbf{f}$. By the definition of the action and the Serre relations in $\mathbf{U}$,

$$
s_{i j}^{\prime} y=\left(\sum_{t=0}^{b_{i j}}(-1)^{t} \pi_{i}^{\left(\begin{array}{l}
t \\
2
\end{array}\right)+t p(j)}\left[\begin{array}{c}
b_{i j} \\
t
\end{array}\right] \theta_{i}^{b_{i j}-t} \theta_{j} \theta_{i}^{t}\right) y=0 .
$$

For $s_{i j}$, we may assume that $y$ is a monomial in the generators $\theta_{i}$ for $i \in I$, so $y=m(f) 1$ where $m(f) \in \mathbf{B}^{\prime}$ is a monomial in the $f_{i}$ for $i \in I$. By repeated application of Lemma $4.2, s_{i j} m(f)=c m(f) s_{i j}$ for some scalar $c \in \mathbb{Q}(q)^{\pi}$. Since $\overline{{ }_{k} r}(1)=0$ for all $k \in I, s_{i j} 1=0$ whence $s_{i j} y=0$. 
Corollary 4.8. As $\mathbf{B}$-modules, $\mathbf{f} \cong \mathbf{B} / \sum_{i} \mathbf{B} e_{i}$.

Proof. The final remark in the proof of Proposition 4.7 shows that there is a B-module homomorphism $\mathbf{B} / \sum_{i} \mathbf{B} e_{i} \rightarrow \mathbf{f}$. On the other hand, the $f_{i}$ generate a subalgebra of $\mathbf{B}$ isomorphic to $\mathbf{f}$, so this map must be an isomorphism.

Proposition 4.9. There is a unique bilinear form $(-,-)_{P}$ on $\mathbf{f}$ satisfying

$$
(1,1)_{P}=1, \quad\left(f_{i} y, z\right)_{P}=\left(y, e_{i} z\right)_{P} \quad \forall y, z \in \mathbf{f}, i \in I .
$$

Moreover, this bilinear form is symmetric.

Proof. Note that we have

$$
\left(\theta_{i} y, z\right)_{P}=\left(f_{i} y, z\right)_{P}=\left(y, e_{i} z\right)_{P}=\left(y,{ }_{i} r z\right)_{P}
$$

and we already have a unique bilinear form satisfying this requirement; namely, $(y, z)_{P}=\mathbf{n}(|y|)\{x, y\}$ where $\mathbf{n}\left(\sum_{i \in I} \nu_{i} i\right)=\prod_{i \in I}\left\{\theta_{i}, \theta_{i}\right\}^{-\nu_{i}}$.

Corollary 4.10. The bilinear form $(-,-)_{P}$ on $\mathbf{f}$ is nondegenerate; moreover, we have $\left(\mathbf{f}_{\nu}, \mathbf{f}_{\mu}\right)_{P}=0$ if $\nu \neq \mu$.

The bilinear form $(-,-)_{P}$ will be referred to as the polarization on $\mathbf{f}$. Corollary 2.10 implies the following.

We define a category $\mathcal{P}$ as follows. The objects of $\mathcal{P}$ are $\mathbf{B}$-modules $M$ such that for any $m \in M$, there exists an $t \in \mathbb{N}$ such that for any $i_{1}, \ldots, i_{t} \in I, e_{i_{1}} \ldots e_{i_{t}} m=0$. The homomorphisms are B-module homomorphisms. Then we have $\mathbf{f},\left.\mathbf{f}\right|_{\pi= \pm 1} \in \mathcal{P}$. In fact, $\left.\mathbf{f}\right|_{\pi= \pm 1}$ are the only simple modules up to isomorphism and $\mathcal{P}$ is semisimple.

Lemma 4.11. Let $M \in \mathcal{P}$. For each $i \in I$, every $m \in M$ has a unique expression of the form

$$
m=\sum_{t \geq 0} f_{i}^{(t)} m_{t}
$$

where $m_{t} \in \operatorname{ker} e_{i}$ and $m_{t}$ is nonzero for finitely many $t$. We will refer to this as its i-string decomposition. 
Proof. By the definition of $\mathcal{P}$, each $e_{i}$ is locally finite on $M$. Note that $e_{i}$ and $f_{i}$ generate a subalgebra of $\mathbf{B}$ isomorphic to $\mathbf{F}_{q_{i}, \pi_{i}}$ and so Lemma 4.1(ii) finishes the proof.

\subsection{Crystal bases}

Let $i \in I$. Let $M \in \mathcal{P}$ and $m \in M$ such that $m=\sum_{t} f_{i}^{(t)} m_{t}$ with $m_{t} \in \operatorname{ker} e_{i}$. We define the Kashiwara operators

$$
\tilde{e}_{i} m=\sum_{t} f_{i}^{(t-1)} m_{t}, \quad \text { and } \tilde{f}_{i} m=\sum_{t} f_{i}^{(t+1)} m_{t}
$$

Note that these operators (super)commute with B-module homomorphisms.

Let $\mathcal{A} \subseteq \mathbb{Q}(q)^{\pi}$ be the subring of functions regular at $q=0$.

Definition 4.12. A free $\mathcal{A}$-submodule $L$ of $\mathbf{B}$-module $M$ which is free as a $\mathbb{Q}(q)^{\pi}$ module in the category $\mathcal{P}$ is called a crystal lattice if

1. $L \otimes_{\mathcal{A}} \mathbb{Q}(q)^{\pi}=M$;

2. $\tilde{e}_{i} L \subseteq L$ and $\tilde{f}_{i} L \subseteq L$.

(Note that $L / q L$ is a free $\mathbb{Q}^{\pi}$-module.) $M$ is said to have a crystal basis $(L, B)$ if a subset $B$ of $L / q L$ satisfies

(3) $B$ is a $\pi$-basis of $L / q L$ (in the sense of Definition 3.41);

(4) $\tilde{e}_{i} B \subseteq B \cup\{0\}$ and $\tilde{f}_{i} B \subseteq B$;

(5) For $b \in B$, if $\tilde{e}_{i} b \neq 0$ then $b=\tilde{f}_{i} \tilde{e}_{i} b$.

Remark 4.13. We can similarly define the notion of crystal basis to $\left.\mathbf{B}\right|_{ \pm 1}$-modules by specializing the above definition similarly. In particular, a crystal basis with respect to $\left.\mathbf{B}\right|_{\pi=1}$ is precisely the same as the crystal basis defined in [Kas1]. 
We let $\mathcal{L}$ be the $\mathcal{A}$-submodule of $\mathbf{f}$ generated by all elements of the form $\tilde{f}_{i_{1}} \ldots \tilde{f}_{i_{t}} 1$. We let $\mathcal{B}=\left\{\pi^{\epsilon} \tilde{f}_{i_{1}} \ldots \tilde{f}_{i_{t}} 1:\left(i_{1}, \ldots, i_{n}\right) \in I^{n}, n \geq 0, \epsilon \in\{0,1\}\right\}$ be the subset of $\mathcal{L} / q \mathcal{L}$. We shall prove that $(\mathcal{L}, \mathcal{B})$ is a crystal basis of $\mathbf{f}$. Note that the bilinear form allows us to define a dual lattice in $\mathbf{f}$ as follows:

$$
\mathcal{L}^{\vee}=\left\{x \in \mathbf{f} \mid(x, \mathcal{L})_{P} \subseteq \mathcal{A}\right\}
$$

There are analogous constructions for $\mathbf{U}$-modules which we will develop now.

Lemma 4.14. Let $M \in \mathcal{O}$. For each $i \in I$, every $m \in M_{\lambda}$ has a unique expression of the form

$$
m=\sum_{t \geq 0} F_{i}^{(t)} m_{t}
$$

where $m_{t} \in M_{\lambda+t i^{\prime}} \cap$ ker $E_{i}$ are nonzero for finitely many $t$. We will refer to this as its i-string decomposition.

Proof. This lemma is known when $p(i)=0$; see [Kas1, §2.2]. When $p(i)=1, M$ is a direct sum of simple $\mathbf{U}(i)$-modules, where $\mathbf{U}(i)$ is the quantum group of $\mathfrak{o s p}(1 \mid 2)$; see $[\mathrm{ClW}]$. This proves existence. Uniqueness is proved similarly to the case when $p(i)=0$.

Definition 4.15. Let $m \in M_{\lambda}$ with

$$
m=\sum_{t \geq 0} F_{i}^{(t)} m_{t}
$$

where $m_{t} \in M_{\lambda+t i^{\prime}} \cap$ ker $e_{i}$ are nonzero for finitely many $t$. We define

$$
\tilde{e}_{i} m=\sum_{t} F_{i}^{(t-1)} m_{t}, \quad \tilde{f}_{i} m=\sum_{t} F_{i}^{(t+1)} m_{t} .
$$

Note that $\tilde{e}_{i} m \in M_{\lambda+i^{\prime}}$ and $\tilde{f}_{i} m \in M^{\lambda-i^{\prime}}$. Moreover, $\tilde{e}_{i}$ and $\tilde{f}_{i}$ (super)commute with U-module homomorphisms.

Definition 4.16. Let $M$ be a $\pi$-free $\mathbf{U}$-module in the category $\mathcal{O}$. A free $\mathcal{A}$-submodule $L$ of $M$ is called a crystal lattice of $M$ if 
1. $L \otimes_{\mathcal{A}} \mathbb{Q}(q)^{\pi}=M$;

2. $L=\bigoplus_{\lambda \in P} L_{\lambda}$ where $L_{\lambda}=L \cap M_{\lambda}$ for all $\lambda \in P$;

3. $\tilde{e}_{i} L \subseteq L$ and $\tilde{f}_{i} L \subseteq L$.

A pair $(L, B)$ is called a crystal basis of $M$ if a subset $B$ of the $\mathbb{Q}^{\pi}$-module $L / q L$ satisfies

(4) $B$ is a $\pi$-basis of $L / q L$ over $\mathbb{Q}^{\pi}$;

(5) $B=\bigsqcup_{\lambda \in P} B_{\lambda}$ where $B_{\lambda}=B \cap L_{\lambda} / q L_{\lambda}$,

(6) $\tilde{e}_{i} B \subseteq B \cup\{0\}$ and $\tilde{f}_{i} B \subseteq B \cup\{0\}$;

(7) For $b, b^{\prime} \in B, \tilde{e}_{i} b=b^{\prime}$ if and only if $b=\tilde{f}_{i} b^{\prime}$.

Also a $\pi= \pm 1$ version of crystal basis for $\left.\mathbf{U}\right|_{\pi= \pm 1}$ integrable modules can be formulated similarly as in Remark 4.13.

Remark 4.17. We shall set out to prove the existence of the crystal bases for the $\pi$-free integrable modules $V(\lambda)$, for $\lambda \in X^{+}$. Assume for the moment that we have done this. Since these axioms are unaffected under direct sums of lattices and parity changes, we can endow any $\pi$-free module $M \in \mathcal{O}_{\text {int }}$ with a crystal basis built out of direct sums of the simples. (In fact, by specializing the crystal bases at $\pi= \pm 1$, we see that any integrable module in $\mathcal{O}_{\mathrm{int}}$ can be endowed with a crystal basis in a more general sense.) Uniqueness of a maximal crystal basis on $M$ (up to isomorphism) can be proved by the same arguments as in [Kas1, §2.6].

Example 4.18. Let $I=\{i\}$. Then the simple modules are $(n+1)$-dimensional modules $V(n)$ for $n \in \mathbb{Z}_{\geq 0}$, which are generated by the highest weight vectors $\eta_{n}$. Define the $\mathcal{A}$-lattice $\mathcal{L}(n)=\bigoplus_{k=0}^{n} \mathcal{A} F^{(k)} \eta_{n}$ in $V(n)$, and define the set $\mathcal{B}(n)=$ $\left\{\pi^{\epsilon} F^{(k)} \eta_{n}+q \mathcal{L}(n) \mid 0 \leq k \leq n, \epsilon \in\{0,1\}\right\}$ (the index $i$ is suppressed here). Then $(\mathcal{L}(n), \mathcal{B}(n))$ is a crystal basis of $V(n)$. In this case, $\mathcal{B}(n)$ is actually a genuine $\mathbb{Q}^{\pi}$-basis for $\mathcal{L}(n) / q \mathcal{L}(n)$. 
Example 4.19. Let $\lambda \in P^{+}$and recall $\eta_{\lambda}$ is a highest weight vector of $V(\lambda)$. Consider the subset $\mathcal{B}(\lambda):=\left\{\pi^{\epsilon} \tilde{f}_{i_{1}} \ldots \tilde{f}_{i_{t}} \eta_{n}:\left(i_{1}, \ldots, i_{n}\right) \in I^{n}, n \geq 0, \epsilon \in\{0,1\}\right\} \backslash\{0\}$ of $V(\lambda)$. Let $\mathcal{L}(\lambda)$ be the $\mathcal{A}$-submodule of $V(\lambda)$ generated by $\mathcal{B}(\lambda)$. We shall prove that $(\mathcal{L}(\lambda), \mathcal{B}(\lambda))$ is a crystal basis.

Example 4.20. Assume that $I_{1}$ contains $i, j$ such that $a_{i j}=a_{j i}=0$. Then $\tilde{f}_{i} \tilde{f}_{j}=$ $\pi \tilde{f}_{j} \tilde{f}_{i}$. In particular, this demonstrates why we should expect a $\pi$-basis as opposed to $a \mathbb{Q}(q)^{\pi}$-basis.

\subsection{Tensor Product Rule}

We will demonstrate a rule to define the tensor product of crystal bases. We will first do this in rank 1 , so suppose for the moment that $I=\{i\}$ with $p(i)=1$ and let us suppress the subscripts on the generators of $\mathbf{U}$. We note that $\Delta(E)=E \otimes K^{-1}+1 \otimes E$ and $\Delta(F)=F \otimes 1+J K \otimes F$.

Let $n \in \mathbb{Z}_{\geq 0}$ and We consider the module $V(n) \otimes V(1)$. This module has two

submodules over $\mathbb{Q}(q)^{\pi}$ generated by singular vectors: a submodule $N_{1}$ generated by the (even) singular vector

$$
w=\eta_{n} \otimes \eta_{1}
$$

and $N_{2}$ generated by the (odd) singular vector

$$
z=\eta_{n} \otimes F \eta_{1}-q[n]_{q, \pi}^{-1} F \eta_{n} \otimes \eta_{1}
$$

We directly compute

$$
\begin{aligned}
F^{(k)} w & =F^{(k)} \eta_{n} \otimes \eta_{1}+(\pi q)^{n+1-k} F^{(k-1)} \eta_{n} \otimes F \eta_{1} \\
F^{(k)} z & =\left(1-(\pi q)^{n-k}[n]_{q, \pi}^{-1}[k]_{q, \pi}\right) F^{(k)} \eta_{n} \otimes F \eta_{1}-q[n]_{q, \pi}^{-1}[k+1]_{q, \pi} F^{(k+1)} \eta_{n} \otimes \eta_{1}
\end{aligned}
$$

Observing that $\pi(\pi q)^{n-k}[n]_{q, \pi}^{-1}[k]_{q, \pi} \in q^{2 n-2 k} \mathcal{A}$, we have

$$
F^{(k)} w=\left\{\begin{array}{ll}
F^{(k)} \eta_{n} \otimes \eta_{1} & \text { if } 0 \leq k<n+1 \\
F^{(n)} \eta_{n} \otimes F \eta_{1} & \text { if } k=n+1
\end{array} \quad \bmod q \mathcal{L}\right.
$$


and

$$
F^{(k)} z=F^{(k)} \eta_{n} \otimes F \eta_{1} \quad \bmod q \mathcal{L}, \quad 0 \leq k \leq n-1 .
$$

In particular, $V(n) \otimes V(1) \cong N_{1} \oplus N_{2} \cong V(n+1) \oplus V(n-1)$.

The above calculations remain to make perfect sense for $\left.\left.V(n)\right|_{\pi= \pm 1} \otimes V(1)\right|_{\pi= \pm 1}$, with $\pi$ in the formulas above interpreted as 1 and -1 accordingly. In particular,

$$
\left.\left.\left.\left.V(n)\right|_{\pi= \pm 1} \otimes V(1)\right|_{\pi= \pm 1} \cong N_{1} \oplus N_{2} \cong V(n+1)\right|_{\pi= \pm 1} \oplus V(n-1)\right|_{\pi= \pm 1}
$$

We can use these calculations to prove a tensor product rule for crystal bases in general, so let $I$ be arbitrary. Let $M$ be an integrable $\mathbf{U}$-module with crystal basis $(\mathcal{L}, B)$. For each $i \in I$ and $b \in B$, define

$$
\begin{aligned}
& \varphi_{i}(b)=\max \left\{n \mid \tilde{f}_{i}^{n} b \neq 0\right\}, \\
& \varepsilon_{i}(b)=\max \left\{n \mid \tilde{e}_{i}^{n} b \neq 0\right\} .
\end{aligned}
$$

We note that $\varphi_{i}(b)=\left\langle\alpha_{i}^{\vee}, \mu\right\rangle+\varepsilon_{i}(b)$ for $b \in B_{\mu}$.

Theorem 4.21. Let $M, M^{\prime} \in \mathcal{O}_{\text {int }}$ be modules with crystal bases $(L, B)$ and $\left(L^{\prime}, B^{\prime}\right)$. Let $B \otimes B^{\prime}=\left\{b \otimes b^{\prime} \in(L / q L) \otimes_{\mathbb{Q}^{\pi}}\left(L^{\prime} / q L^{\prime}\right): b \in B, b^{\prime} \in B^{\prime}\right\}$. The tensor product $M \otimes M^{\prime}$ has a crystal basis $\left(L \otimes_{\mathcal{A}} L^{\prime}, B \otimes B^{\prime}\right)$ subject to the rules:

$$
\begin{aligned}
& \tilde{f}_{i}\left(b \otimes b^{\prime}\right)= \begin{cases}\tilde{f}_{i} b \otimes b^{\prime} & \text { if } \varphi_{i}(b)>\varepsilon_{i}\left(b^{\prime}\right), \\
\pi_{i}^{p(b)+\langle i,|b|\rangle} b \otimes \tilde{f}_{i} b^{\prime} & \text { otherwise; }\end{cases} \\
& \tilde{e}_{i}\left(b \otimes b^{\prime}\right)= \begin{cases}\pi_{i}^{p(b)+\langle i,|b|\rangle} b \otimes \tilde{e}_{i} b^{\prime} & \text { if } \varphi_{i}(b)<\varepsilon_{i}\left(b^{\prime}\right), \\
\tilde{e}_{i} b \otimes b^{\prime} & \text { otherwise. }\end{cases}
\end{aligned}
$$

(All equalities are understood in $L \otimes_{\mathcal{A}} L^{\prime} / q L \otimes_{\mathcal{A}} L^{\prime}$.)

Proof. It is sufficient to prove this for a fixed $i$, in which case the theorem reduces to a statement for $I=\{i\}$. When $p(i)=0$, the theorem is [Kas1, Theorem 1]. Assume $p(i)=1$. Since modules are completely reducible, it suffices to prove this 
for tensor products of simple modules $V(n) \otimes V(m)$, by induction on $m$. Recall from Example 4.18 that $(\mathcal{L}(n), \mathcal{B}(n))$ is a crystal basis for $V(n)$.

From the odd rank 1 calculations, the theorem holds for $V(n) \otimes V(1)$. This takes care the base case of induction.

Now assume the theorem holds for $\left.\left.V(n)\right|_{\pi=-1} \otimes V(m)\right|_{\pi=-1}$. Note that

$$
V(n) \otimes V(m) \otimes V(1) \cong V(n) \otimes(V(m+1) \oplus V(m-1))
$$

By the complete reducibility and the base case proved above, we conclude that $(\mathcal{L}(n) \otimes$ $\mathcal{L}(m) \otimes \mathcal{L}(1), \mathcal{B}(n) \otimes \mathcal{B}(m) \otimes \mathcal{B}(1))$ is a crystal basis of $V(n) \otimes V(m) \otimes V(1)$. Moreover, $(\mathcal{L}(n) \otimes \mathcal{L}(m) \otimes \mathcal{L}(1), \mathcal{B}(n) \otimes \mathcal{B}(m) \otimes \mathcal{B}(1))$ decomposes as $(\mathcal{L}(n) \otimes(\mathcal{L}(m+1) \oplus \mathcal{L}(m-$ $1)), \mathcal{B}(n) \otimes(\mathcal{B}(m+1) \cup \mathcal{B}(m-1)))$. Therefore, $(\mathcal{L}(n) \otimes \mathcal{L}(m+1), \mathcal{B}(n) \otimes \mathcal{B}(m+1))$ is a crystal basis of $V(n) \otimes V(m+1)$.

Finally, the tensor product rules are proved by directly computing the action of $\tilde{f}, \tilde{e}$ on each element of $(\mathcal{B}(n) \otimes \mathcal{B}(m)) \otimes \mathcal{B}(1)$ and rewriting it in terms of $\mathcal{B}(n) \otimes$ $(\mathcal{B}(m) \otimes \mathcal{B}(1))$. The key feature is that the power of $\pi$ in the tensor product rule is $\mathbb{Z}$-linear in $|b|$.

As one might expect, the tensor product rule is dependent upon the choice of coproduct. In fact, as shown in [CHW2], it takes the following more natural form if we consider $\Delta^{\prime}$-induced module structure instead.

Theorem 4.22. Let $M, M^{\prime} \in \mathcal{O}_{\text {int }}$ be modules with crystal bases $(L, B)$ and $\left(L^{\prime}, B^{\prime}\right)$. Let $B \otimes B^{\prime}=\left\{b \otimes b^{\prime} \in(L / q L) \otimes_{\mathbb{Q}^{\pi}}\left(L^{\prime} / q L^{\prime}\right): b \in B, b^{\prime} \in B^{\prime}\right\}$. The tensor product $M \otimes^{\prime} M^{\prime}$ has a crystal basis $\left(L \otimes_{\mathcal{A}} L^{\prime}, B \otimes B^{\prime}\right)$ subject to the rules:

$$
\begin{aligned}
& \tilde{f}_{i}\left(b \otimes b^{\prime}\right)= \begin{cases}\tilde{f}_{i} b \otimes b^{\prime} & \text { if } \varphi_{i}(b)>\varepsilon_{i}\left(b^{\prime}\right), \\
\pi_{i}^{p(b)} b \otimes \tilde{f}_{i} b^{\prime} & \text { otherwise; }\end{cases} \\
& \tilde{e}_{i}\left(b \otimes b^{\prime}\right)= \begin{cases}\pi_{i}^{p(b)} b \otimes \tilde{e}_{i} b^{\prime} & \text { if } \varphi_{i}(b)<\varepsilon_{i}\left(b^{\prime}\right), \\
\tilde{e}_{i} b \otimes b^{\prime} & \text { otherwise. }\end{cases}
\end{aligned}
$$

(All equalities are understood in $L \otimes_{\mathcal{A}} L^{\prime} / q L \otimes_{\mathcal{A}} L^{\prime}$.) 


\subsection{Polarization}

Let $\tau_{1}: \mathbf{U} \rightarrow \mathbf{U}$ be the anti-automorphism defined by $\tau_{1}\left(E_{i}\right)=q_{i}^{-1} \tilde{K}_{i}^{-1} F_{i}, \tau_{1}\left(F_{i}\right)=q_{i}^{-1} \tilde{K}_{i} E_{i}, \tau_{1}\left(K_{\mu}\right)=K_{\mu}, \tau_{1}\left(J_{\mu}\right)=J_{\mu}, \quad\left(i \in I, \mu \in P^{\vee}\right)$ such that $\tau_{1}(x y)=\tau_{1}(y) \tau_{1}(x)$ for $x, y \in \mathbf{U}$. One checks that $\tau_{1}^{2}=1$.

Proposition 4.23. Let $\lambda \in X^{+}$. There is a unique bilinear form $(-,-)$on $V(\lambda)$, which satisfies $\left(\eta_{\lambda}, \eta_{\lambda}\right)=1$ and

$$
(u v, w)=\left(v, \tau_{1}(u) w\right), \quad \forall u \in \mathbf{U}, v, w \in V(\lambda) .
$$

Moreover, this bilinear form on $V(\lambda)$ is symmetric.

Recall the $\mathcal{A}$-lattices $\mathcal{L}(\lambda)$ of $V(\lambda)$ from Example 4.19. We define the dual lattices in $V(\lambda)$ to be

$$
\mathcal{L}(\lambda)^{\vee}=\{v \in V(\lambda) \mid(v, \mathcal{L}(\lambda)) \subseteq \mathcal{A}\}
$$

For a weight $\mathbf{U}$-module $M$, we call a bilinear form $(-,-)$ on $M$ a polarization if (4.10) is satisfied with $M$ in place of $V(\lambda)$. Note that if $m \in M_{\lambda}$ and $m^{\prime} \in M_{\mu}$, then

$$
\left(m, m^{\prime}\right)=0 \text { unless } \lambda=\mu \text { and } p(m)=p\left(m^{\prime}\right) .
$$

Recall [Kas1, Lemma 2.5.1] that the tensor product of modules admitting polarizations also admits a natural polarization given by the tensor of the bilinear forms. In our super setting, this is not quite true due to the additional asymmetry in the definition of the coproduct. However, this can be resolved by requiring the $\Delta$ and $\Delta^{\prime}$ actions to be adjoint.

Lemma 4.24. Assume $M, N \in \mathcal{O}_{\mathrm{int}}$ admit polarizations (-,-). Then the symmetric bilinear form on the module $M \otimes N$ given by $\left(m_{1} \otimes n_{1}, m_{2} \otimes n_{2}\right)=\left(m_{1}, m_{2}\right)\left(n_{1}, n_{2}\right)$ satisfies

$$
\left(\Delta(u)\left(m_{1} \otimes m_{2}\right), n_{1} \otimes n_{2}\right)=\left(m_{1} \otimes m_{2}, \Delta^{\prime}\left(\tau_{1}(u)\right)\left(n_{1} \otimes n_{2}\right)\right),
$$

for $u \in \mathbf{U}, m_{1}, m_{2} \in M, n_{1}, n_{2} \in N$. 
We call such a bilinear form on $M \otimes N$ a $J$-polarization, as the difference on $\Delta$ and $\Delta^{\prime}$ is caused by the $J_{\mu}$ 's.

Proof. This follows from direct computation. For example, we shall verify this for $u=F_{i}$. Let $m_{1} \in M_{\mu}$ and $m_{2} \in M_{\mu^{\prime}}$. By a direct computation, we have

$$
\begin{aligned}
\left(\Delta\left(F_{i}\right)\left(m_{1} \otimes n_{1}\right), m_{2} \otimes n_{2}\right)= & \left(F_{i} m_{1}, m_{2}\right)\left(n_{1}, n_{2}\right) \\
& +\pi_{i}^{p\left(m_{1}\right)}\left(\pi_{i} q_{i}\right)^{\langle i, \mu\rangle}\left(m_{1}, m_{2}\right)\left(F_{i} n_{1}, n_{2}\right),
\end{aligned}
$$

and

$$
\begin{aligned}
\left(m_{1} \otimes n_{1}, \Delta^{\prime}\left(q_{i}^{-1} K_{i} E_{i}\right)\left(m_{2} \otimes n_{2}\right)\right)= & \left(m_{1}, q_{i}^{-1} K_{i} E_{i} m_{2}\right)\left(n_{1}, n_{2}\right) \\
& +\pi_{i}^{p\left(m_{2}\right)}\left(\pi_{i} q_{i}\right)^{\left\langle\alpha_{i}^{\vee}, \mu^{\prime}\right\rangle}\left(m_{1}, m_{2}\right)\left(n_{1}, q_{i}^{-1} K_{i} E_{i} n_{2}\right) .
\end{aligned}
$$

By (4.11) and the definition of a polarization, (4.12) and (4.13) are equal.

Remark 4.25. To see why we need $\Delta^{\prime}$ in Lemma 4.24, we compute using $\Delta$ in replace of $\Delta^{\prime}$ that

$$
\begin{aligned}
\left(m_{1} \otimes n_{1},\right. & \left.\Delta\left(\tau_{1}\left(E_{i}\right)\right)\left(m_{2} \otimes n_{2}\right)\right) \\
& =\left(m_{1}, \tau_{1}\left(E_{i}\right) m_{2}\right)\left(n_{1}, n_{2}\right)+\pi_{i}^{p\left(m_{2}\right)} q_{i}^{\left\langle\alpha_{i}^{\vee}, \mu^{\prime}\right\rangle}\left(m_{1}, m_{2}\right)\left(n_{1}, \tau_{1}\left(E_{i}\right) n_{2}\right) .
\end{aligned}
$$

In particular, if $p(i)=1$ and $\left\langle\alpha_{i}^{\vee}, \mu^{\prime}\right\rangle \notin 2 \mathbb{Z}$, then (4.14) is not equal to (4.12).

For $\lambda, \mu \in X^{+}$, we define the $\mathbf{U}$-module homomorphisms

$$
\begin{array}{cc}
\chi(\lambda, \mu): V(\lambda+\mu) \rightarrow V(\lambda) \otimes V(\mu), & \eta_{\lambda+\mu} \mapsto \eta_{\lambda} \otimes \eta_{\mu}, \\
\chi^{\prime}(\lambda, \mu): V(\lambda+\mu) \rightarrow V(\lambda) \otimes^{\prime} V(\mu), & \eta_{\lambda+\mu} \mapsto \eta_{\lambda} \otimes \eta_{\mu}, \\
\gamma(\lambda, \mu): V(\lambda) \otimes V(\mu) \rightarrow V(\lambda+\mu), & \eta_{\lambda} \otimes \eta_{\mu} \mapsto \eta_{\lambda+\mu}, \\
\gamma^{\prime}(\lambda, \mu): V(\lambda) \otimes^{\prime} V(\mu) \rightarrow V(\lambda+\mu), & \eta_{\lambda} \otimes \eta_{\mu} \mapsto \eta_{\lambda+\mu} .
\end{array}
$$

Then $\gamma(\lambda, \mu) \circ \chi(\lambda, \mu)$ and $\gamma^{\prime}(\lambda, \mu) \circ \chi^{\prime}(\lambda, \mu)$ are the identity map on $V(\lambda+\mu)$. In particular, $V(\lambda) \otimes V(\mu)=\operatorname{im} \chi \oplus \operatorname{ker} \gamma$ and $V(\lambda) \otimes^{\prime} V(\mu)=\operatorname{im} \chi^{\prime} \oplus \operatorname{ker} \gamma^{\prime}$. Note that these maps, being $\mathbf{U}$-module homomorphisms, commute with Kashiwara operators.

A key property of these maps is the following adjointness statement. 
Lemma 4.26. Let $\lambda, \mu \in X^{+}$. Let $(-,-)$denote the polarization on $V(\lambda+\mu)$ and the $J$-polarization on $V(\lambda) \otimes V(\mu)$. Then we have

$$
(\gamma(\lambda, \mu)(w), v)=\left(w, \chi^{\prime}(\lambda, \mu)(v)\right), \quad\left(\gamma^{\prime}(\lambda, \mu)(w), v\right)=(w, \chi(\lambda, \mu)(v))
$$

for $v \in V(\lambda+\mu)$ and $w \in V(\lambda) \otimes V(\mu)$.

\subsection{Main statements and grand loop argument}

Recall the definitions of $\mathcal{L}, \mathcal{L}(\lambda), \mathcal{B}, \mathcal{B}(\lambda)$ given in $\S 4.2$. We are now ready to formulate the main theorems on crystal bases for $\mathbf{f}$ and integrable modules $V(\lambda)$.

Theorem 4.27. $(\mathcal{L}, \mathcal{B})$ is a crystal basis of $\mathbf{f}$. Moreover, let $x=\sum_{n \geq 0} \theta_{i}^{(n)} x_{n}$ be the $i$-string decomposition of $x \in \mathbf{f}$. Then,

1. $x \in \mathcal{L}$ if and only if $x_{n} \in \mathcal{L}$ for all $n$.

2. If $x+q \mathcal{L} \in \mathcal{B}$, then $x=\theta_{i}^{(n)} x_{n} \bmod q \mathcal{L}$ for some $n$ and $x_{n}+q \mathcal{L} \in \mathcal{B}$.

3. If $\tilde{e}_{j} x=0$ for all $j \in I$ then $x=0$; if $\tilde{e}_{j} x \neq 0$ then $\tilde{f}_{j} \tilde{e}_{j} x=x$.

Theorem 4.28. Let $\lambda \in X^{+}$. Then $(\mathcal{L}(\lambda), \mathcal{B}(\lambda))$ is a crystal basis of $V(\lambda)$.

Recall that we may view $\mathbf{f}$ naturally as a $\mathbf{U}^{-}$-module. For $\lambda \in X^{+}$, we define the $\mathbf{U}^{-}$-linear projection map

$$
\wp_{\lambda}: \mathbf{f} \longrightarrow V(\lambda), \quad 1 \mapsto \eta_{\lambda}
$$

Theorem 4.29. Let $\lambda \in X^{+}$.

1. $\wp_{\lambda}(\mathcal{L})=\mathcal{L}(\lambda)$

(This induces a homomorphism $\hat{\wp}_{\lambda}: \mathcal{L} / q \mathcal{L} \rightarrow \mathcal{L}(\lambda) / q \mathcal{L}(\lambda)$.)

2. $\hat{\wp}_{\lambda}$ sends $\left\{b \in \mathcal{B} \mid \hat{\wp}_{\lambda}(b) \neq 0\right\}$ isomorphically to $\mathcal{B}(\lambda)$;

3. if $b \in \mathcal{B}$ satisfies $\hat{\wp}_{\lambda}(b) \neq 0$, then $\tilde{e}_{i} \hat{\wp}_{\lambda}(b)=\hat{\wp}_{\lambda}\left(\tilde{e}_{i} b\right)$. 
These statements are proved in [CHW2] using a modified version of Kashiwara's grand loop argument (cf. [Kas1, §4]). For $\lambda, \mu \in X^{+}$, recall the maps $\gamma(\lambda, \mu)$ and $\chi(\lambda, \mu)$ from (4.15). We also define a map

$$
S(\lambda, \mu): V(\lambda) \otimes V(\mu) \longrightarrow V(\lambda)
$$

by $S(\lambda, \mu)\left(u \otimes \eta_{\mu}\right)=u$ and $S(\lambda, \mu)\left(V(\lambda) \otimes \sum f_{i} V(\mu)\right)=0$. This is a $\mathbf{U}^{-}$-linear map. Therefore, we have a $\mathbf{U}^{-}$-linear map $S(\lambda, \mu) \circ \chi(\lambda, \mu): V(\lambda+\mu) \longrightarrow V(\lambda)$ sending $\eta_{\lambda+\mu}$ to $\eta_{\lambda}$.

Then let $\Lambda(l)=\{\zeta \in \Lambda:$ ht $\zeta \leq l\}$.

Theorem 4.30. Let $C_{l}$ be the collection of the following statements.

$\left(C_{l} .1\right)$ For $\zeta \in \Lambda(l)$,

$$
\tilde{e}_{i} \mathcal{L}_{\zeta} \subseteq \mathcal{L}
$$

$\left(C_{l} .2\right)$ For $\zeta \in \Lambda(l)$ and $\lambda \in X^{+}$,

$$
\tilde{e}_{i} \mathcal{L}(\lambda)_{\lambda-\zeta^{\prime}} \subset \mathcal{L}(\lambda)
$$

$\left(C_{l} .3\right)$ For $\zeta \in \Lambda(l)$ and $\lambda \in X^{+}, \wp_{\lambda} \mathcal{L}_{\zeta}=\mathcal{L}(\lambda)_{\lambda-\zeta^{\prime}}$.

$\left(C_{l}\right.$.4) For $\zeta \in \Lambda(l), \mathcal{B}_{\zeta}$ is a $\pi$-basis of $\mathcal{L}_{\zeta} / q \mathcal{L}_{\zeta}$.

$\left(C_{l} .5\right)$ For $\zeta \in \Lambda(l)$ and $\lambda \in X^{+}, \mathcal{B}(\lambda)_{\lambda-\zeta^{\prime}}$ is a $\pi$-basis of $\mathcal{L}(\lambda)_{\lambda-\zeta^{\prime}} / q \mathcal{L}(\lambda)_{\lambda-\zeta^{\prime}}$.

$\left(C_{l} .6\right)$ For $\zeta \in \Lambda(l-1)$ and $\lambda \in X^{+}, \tilde{f}_{i}\left(x \eta_{\lambda}\right) \equiv\left(\tilde{f}_{i} x\right) \eta_{\lambda} \bmod q \mathcal{L}(\lambda)$ for $x \in \mathcal{L}_{\zeta}$.

$\left(C_{l} .7\right)$ For $\zeta \in \Lambda(l)$ and $\lambda \in X^{+}, \tilde{e}_{i} \mathcal{B}_{\zeta} \subset \mathcal{B} \cup\{0\}$ and $\tilde{e}_{i} \mathcal{B}(\lambda)_{\lambda-\zeta^{\prime}} \subset \mathcal{B}(\lambda) \cup\{0\}$.

(Cl.8) For $\zeta \in \Lambda(l)$ and $\lambda, \mu \in X^{+}$,

$$
\chi(\lambda, \mu)\left(\mathcal{L}(\lambda+\mu)_{\lambda+\mu-\zeta^{\prime}}\right) \subset \mathcal{L}(\lambda) \otimes \mathcal{L}(\mu) .
$$

$\left(C_{l} .9\right)$ For $\zeta \in \Lambda(l)$ and $\lambda, \mu \in X^{+}$,

$$
\gamma(\lambda, \mu)\left((\mathcal{L}(\lambda) \otimes \mathcal{L}(\mu))_{\lambda+\mu-\zeta^{\prime}}\right) \subset \mathcal{L}(\lambda+\mu) .
$$


$\left(C_{l} .10\right)$ For $\zeta \in \Lambda(l)$ and $\lambda, \mu \in X^{+}$,

$$
\gamma(\lambda, \mu)\left((\mathcal{B}(\lambda) \otimes \mathcal{B}(\mu))_{\lambda+\mu-\zeta^{\prime}}\right) \subset \mathcal{B}(\lambda+\mu) \cup\{0\} .
$$

(Cl.11) For $\zeta \in \Lambda(l)$ and $\lambda \in X^{+}$

$$
\left\{b \in \mathcal{B}_{\zeta}: \hat{\wp}_{\lambda}(b) \neq 0\right\} \rightarrow \mathcal{B}(\lambda)_{\lambda-\zeta^{\prime}}
$$

is a bijection, where $\hat{\wp}_{\lambda}$ is the map induced by $\wp_{\lambda}$.

$\left(C_{l} .12\right)$ For $\zeta \in \Lambda(l), \lambda \in X^{+}$and $b \in \mathcal{B}_{\zeta}$ such that $\hat{\wp}_{\lambda}(b) \neq 0$, we have

$$
\tilde{e}_{i} \hat{\wp}_{\lambda}(b)=\hat{\wp}_{\lambda}\left(\tilde{e}_{i}(b)\right) \text {. }
$$

$\left(C_{l} .13\right)$ For $\zeta \in \Lambda(l), \lambda \in X^{+} b \in \mathcal{B}(\lambda)_{\lambda-\zeta^{\prime}}$ and $b^{\prime} \in \mathcal{B}(\lambda)_{\lambda-\zeta^{\prime}+i}, b=\tilde{f}_{i} b^{\prime}$ if and only if $b^{\prime}=\tilde{e}_{i} b$.

$\left(C_{l} .14\right)$ For $\zeta \in \Lambda(l)$ and $b \in \mathcal{B}$, if $\tilde{e}_{i} b \neq 0$ then $b=\tilde{f}_{i} \tilde{e}_{i} b$.

Then $C_{l}$ holds for all $l \in \mathbb{N}$.

In the case $I=I_{0}$, Kashiwara (cf. [Kas1]) proved these statements via an induction on $l$. These arguments can be adapted to our super setting, with the main change being book-keeping for the power of $\pi$. We will abstain from stating the proofs here, but the essential changes in the argument are stated in [CHW2]. While the main coproduct used there is our $\Delta^{\prime}$, the statements stay the same except for minor cosmetic changes for Lemma 5.2(2) and the proof of Lemma 5.7 in loc. cit..

\subsection{Further properties of the polarization}

Let us examine more closely the properties of the polarizations on $\mathcal{L}(\lambda)$ and on $\mathcal{L}$. We will say a $\pi$-basis $B$ is $\pi$-orthonormal with respect to a bilinear form $(-,-)$ if $\left(b, b^{\prime}\right)=\pi^{\epsilon_{b, b^{\prime}}} \delta_{b, b^{\prime}}$ for some $\epsilon_{b, b^{\prime}} \in\{0,1\}$. 
Proposition 4.31. Let either (1) $V=\mathbf{f}$ and $(L, B)=(\mathcal{L}, \mathcal{B})$ or (2) $V=V(\lambda)$ and $(L, B)=(\mathcal{L}(\lambda), \mathcal{B}(\lambda))$ for $\lambda \in X^{+}$. Let $(-,-)$be the polarization on $V$ given in $\S 4.1$ or $\$ 4.4$, accordingly. Then,

(i) $(L, L) \subseteq \mathcal{A}$, and so it descends to a bilinear form

$$
(-,-)_{0}: L / q L \times L / q L \rightarrow \mathbb{Q}^{\pi}, \quad(x+q L, y+q L)_{0}=\left.(x, y)\right|_{q=0} .
$$

(ii) $\left(\tilde{f}_{i} u, v\right)_{0}=\pi_{i}^{\epsilon_{i}(u)}\left(u, \tilde{e}_{i} v\right)_{0}$ for $u, v \in \mathcal{L} / q \mathcal{L}$.

(iii) $B$ is a $\pi$-orthonormal $\pi$-basis of $\mathcal{L} / q \mathcal{L}$ with respect to $(-,-)_{0}$.

(iv) $L=\{u \in V ;(u, L) \subseteq \mathcal{A}\}$.

Proof. For notational simplicity and certainty, we will prove the case (2) in detail, while the case (1) is entirely similar.

The same easy reduction as in the proof of [Kas1, Proposition 5.1.1] reduces the proof of Parts (i) and (ii) to the verification of the following identity

$$
\left(\tilde{f}_{i} u, v\right) \equiv \pi_{i}^{\epsilon_{i}(u)}\left(u, \tilde{e}_{i} v\right) \quad \bmod q \mathcal{A}
$$

where $u=F_{i}^{(n)} u_{0} \in \mathcal{L}(\lambda)_{\lambda-\zeta^{\prime}+i^{\prime}}, v=F_{i}^{(m)} v_{0} \in \mathcal{L}(\lambda)_{\lambda-\zeta^{\prime}}$ with $E_{i} u_{0}=E_{i} v_{0}=0$.

To that end, we have the following computation (compare [Kas1, (5.1.2)]):

$$
\begin{aligned}
\left(F_{i}^{(n+1)} u_{0}, F_{i}^{(m)} v_{0}\right) & =\delta_{n+1, m} q_{i}^{m\left\langle i, \lambda-\zeta^{\prime}\right\rangle+m^{2}}\left(E_{i}^{(m)} F_{i}^{(m)} u_{0}, v_{0}\right) \\
& =\delta_{n+1, m} \pi_{i}^{m^{2}+\left(\begin{array}{c}
m+1 \\
2
\end{array}\right)} q_{i}^{m\left\langle i, \lambda-\zeta^{\prime}\right\rangle+m^{2}}\left[\begin{array}{c}
\left\langle i, \lambda-\zeta^{\prime}\right\rangle+2 m \\
m
\end{array}\right]_{q_{i}, \pi_{i}}\left(u_{0}, v_{0}\right) \\
& \equiv \delta_{n+1, m} \pi_{i}^{\left(\begin{array}{c}
m \\
2
\end{array}\right)}\left(u_{0}, v_{0}\right) \bmod q \mathcal{A},
\end{aligned}
$$

where we have used $\left(\begin{array}{c}m+1 \\ 2\end{array}\right)+m^{2} \equiv\left(\begin{array}{c}m \\ 2\end{array}\right)$ mod 2. Therefore $\left(\tilde{f}_{i} u, v\right)_{0}=\pi_{i}^{m-1}\left(u, \tilde{e}_{i} v\right)_{0}$. Since $m-1=n=\epsilon_{i}(u)$, the identity (4.18) follows, and whence (i) and (ii).

Part (iii) follows by induction on weights and using Theorem 4.28 from the identity

$$
\left(b, b^{\prime}\right)_{0}=\left(\tilde{f}_{i} \tilde{e}_{i} b, b^{\prime}\right)_{0}=\left(\tilde{e}_{i} b, \tilde{e}_{i} b^{\prime}\right)_{0},
$$


where $b, b^{\prime} \in B$ and $i \in I$ is chosen such that $\tilde{e}_{i} b \in B$.

To prove (iv), it remains to verify that $\{u \in V ;(u, \mathcal{L}) \subseteq \mathcal{A}\} \subseteq L$ thanks to (i). Denote $\operatorname{sgn}(b)=(b, b)_{0}$. Suppose $u \in V$ is a $\mu$-weight vector such that $(u, L) \subseteq \mathcal{A}$. By Theorem 4.28 and the definition of crystal basis and $\pi$-basis, one can find $B_{\mu}^{0} \subset B_{\mu}$ which is an honest $\mathbb{Q}^{\pi}$-basis for $L_{\mu} / q L_{\mu}$. Then $u$ can be written as $u=\sum_{b \in B_{\mu}^{0}} c_{b} u_{b}$. where $u_{b}+q \mathcal{L}=b$ and $c_{b} \in \mathbb{Q}(q)$. Assume $u \notin L$. Then there exists a minimal $r \in \mathbb{Z}_{>0}$ such that $q^{r} c_{b} \in \mathcal{A}$ for all $b \in B_{\mu}$. Since $(u, L) \subset \mathcal{A}$, we have in particular that $\left(u, \operatorname{sgn}(b) q^{r-1} u_{b}\right) \in \mathcal{A}$. On the other hand, since $\left(u_{b}, u_{b^{\prime}}\right) \in q \mathcal{A}$ for $b \neq b^{\prime}$, we compute that $\left(u, \operatorname{sgn}(b) q^{r-1} u_{b}\right) \in q^{r-1} c_{b}+\mathcal{A}$ for all $b$, whence $q^{r-1} c_{b} \in \mathcal{A}$ for all $b$, contradicting the minimality of $r$. This completes the proof of the proposition.

Remark 4.32. In contrast to the usual quantum group setting in [Kas1, Lu4], $(-,-)_{0}$ here is not positive definite in general, as it could happen that $(b, b)_{0}=\pi$ for some crystal basis element. In particular, the well-known characterization in the usual quantum group setting that an element $u$ lies in the crystal lattice if and only if $(u, u) \in \mathcal{A}$ fails in our super setting in general; see Example 4.33.

Example 4.33. Let $\mathbf{U}$ be the quantum $\mathfrak{o s p}(1 \mid 4)$, with $I=\{\mathbf{1}, \mathbf{2}\}$ (where $\mathbf{1}$ is the odd simple root). Then

$$
\begin{gathered}
p(\mathbf{1})=1, \quad p(\mathbf{2})=0 ; \\
\mathbf{1} \cdot \mathbf{1}=2, \quad \mathbf{1} \cdot \mathbf{2}=\mathbf{2} \cdot \mathbf{1}=-2, \quad \mathbf{2} \cdot \mathbf{2}=4 .
\end{gathered}
$$

Then $\tilde{f}_{\mathbf{1}}^{4} \tilde{f}_{\mathbf{2}} \cdot 1=\theta_{\mathbf{1}}^{(4)} \theta_{\mathbf{2}}$ and $\tilde{f}_{\mathbf{1}}^{3} \tilde{f}_{\mathbf{2}} \tilde{f}_{\mathbf{1}} \cdot 1=\theta_{\mathbf{1}}^{(3)}\left(\theta_{\mathbf{2}} \theta_{\mathbf{1}}-q^{2} \theta_{\mathbf{1}} \theta_{\mathbf{2}}\right)+q^{2} \theta_{\mathbf{1}}^{(4)} \theta_{\mathbf{2}}$ (these will be canonical basis elements as developed in Section 4.8). A direct computation shows that

$$
\left(\theta_{\mathbf{1}}^{(3)}\left(\theta_{\mathbf{2}} \theta_{\mathbf{1}}-q^{2} \theta_{\mathbf{1}} \theta_{\mathbf{2}}\right), \theta_{\mathbf{1}}^{(4)} \theta_{\mathbf{2}}\right)=0
$$

and also

$$
\begin{aligned}
& \left(\theta_{\mathbf{1}}^{(4)} \theta_{\mathbf{2}}, \theta_{\mathbf{1}}^{(4)} \theta_{\mathbf{2}}\right)=(\pi q)^{6}\left([4]_{q, \pi}^{!}\right)^{-1} \in 1+q^{2} \mathbb{Z}^{\pi}[[q]] \\
& \left(\theta_{\mathbf{1}}^{(3)}\left(\theta_{\mathbf{2}} \theta_{\mathbf{1}}-q^{2} \theta_{\mathbf{1}} \theta_{\mathbf{2}}\right), \theta_{\mathbf{1}}^{(3)}\left(\theta_{\mathbf{2}} \theta_{\mathbf{1}}-q^{2} \theta_{\mathbf{1}} \theta_{\mathbf{2}}\right)\right) \\
& =(\pi q)^{3}\left([3]_{q, \pi}^{!}\right)^{-1}\left(1-q^{4}\right) \in \pi+q^{2} \mathbb{Z}^{\pi}[[q]] .
\end{aligned}
$$


It follows that

1. $\left(\tilde{f}_{\mathbf{1}}^{4} \tilde{f}_{\mathbf{2}} \cdot 1, \tilde{f}_{\mathbf{1}}^{4} \tilde{f}_{\mathbf{2}} \cdot 1\right)=1 \bmod q^{2} \mathbb{Z}^{\pi}[[q]] ;$

2. $\left(\tilde{f}_{1}^{3} \tilde{f}_{\mathbf{2}} \tilde{f}_{\mathbf{1}} \cdot 1, \tilde{f}_{1}^{3} \tilde{f}_{\mathbf{2}} \tilde{f}_{1} \cdot 1\right)=\pi \bmod q^{2} \mathbb{Z}^{\pi}[[q]] ;$

3. $\left(\tilde{f}_{\mathbf{1}}^{4} \tilde{f}_{\mathbf{2}} \cdot 1, \tilde{f}_{1}^{3} \tilde{f}_{\mathbf{2}} \tilde{f}_{\mathbf{1}} \cdot 1\right)=q^{2} \bmod q^{4} \mathbb{Z}^{\pi}[[q]]$.

Now (1) and (2) provide us an example that the squared norm of (canonical basis) elements in $\mathcal{B}$ of the same weight do not have uniform sign. Combined with (3), this implies that $\mathcal{L} \subsetneq\left\{u \in \mathbf{f} \mid(u, u)_{P} \in \mathcal{A}\right\}$, since $q^{-1}(1-\pi)\left(\tilde{f}_{1}^{4} \tilde{f}_{2} \cdot 1+\tilde{f}_{\mathbf{1}}^{3} \tilde{f}_{\mathbf{2}} \tilde{f}_{\mathbf{1}} \cdot 1\right)$ belongs to the right-hand side, but not to $\mathcal{L}$.

Now let us compare the bilinear forms on $\mathcal{L}(\lambda)$ and $\mathcal{L}$. Let us introduce the following notation. For $\lambda \in X^{+}$, we say $\lambda \gg 0$ with respect to $\zeta \in \mathbb{N}[I]$ if $\left\langle i, \lambda-\zeta^{\prime}\right\rangle>$ 0 for all $i \in I$.

Proposition 4.34. For given $x, y \in \mathcal{L}_{\zeta}$, take $\lambda \gg 0$ with respect to $\zeta$. Then $\left(x^{-} \eta_{\lambda}, y^{-} \eta_{\lambda}\right)_{0}=(x, y)_{P, 0}$, where $(-,-)_{P, 0}$ is the induced bilinear form on $\mathcal{L} / q \mathcal{L}$.

Proof. This is obvious for $\zeta=0$. We proceed by induction on the height of $\zeta$. We can write $x=\theta_{i} x^{\prime}$ for some $i \in I$. Then

$$
\begin{aligned}
\left(x^{-} \eta_{\lambda}, y^{-} \eta_{\lambda}\right) & =\left(x^{\prime} \eta_{\lambda}, q_{i}^{-1} \tilde{K}_{i} E_{i} y^{-} \eta_{\lambda}\right) \\
& =\left(x^{\prime} \eta_{\lambda}, \frac{\tilde{J}_{i} \tilde{K}_{i i}^{2} r(y)^{-}-\overline{{ }_{i} r}(y)^{-}}{\pi_{i} q_{i}^{2}-1} \eta_{\lambda}\right) \\
& =\frac{\left(\pi_{i} q_{i}^{2}\right)}{\pi q_{i}^{2}-1}\left(x^{\prime-} \eta_{\lambda},{ }_{i} r(y)^{-} \eta_{\lambda}\right)+\frac{1}{1-\pi_{i} q_{i}^{2}}\left(x^{\prime-} \eta_{\lambda},{ }_{i} r(y)^{-} \eta_{\lambda}\right) .
\end{aligned}
$$

Hence, by induction and the assumption $\lambda \gg 0$, we have

$$
\left(x^{-} \eta_{\lambda}, y^{-} \eta_{\lambda}\right)_{0}=\left(x^{-} \eta_{\lambda},{ }_{i} r(y)^{-} \eta_{\lambda}\right)_{0}=\left(x^{\prime}, \overline{r_{i}}(y)\right)_{0}=(x, y)_{0} .
$$

The proposition is proved.

A similar argument directly relates the polarization on modules with the bilinear form $\{-,-\}$. By $\lim _{\lambda \rightarrow \infty}$, we mean the limit as $\min _{i \in I}\langle\lambda, i\rangle \rightarrow \infty$. 
Proposition 4.35. Let $x, y \in \mathbf{f}$. Then $\lim _{\lambda \rightarrow \infty}\left(x^{-} \eta_{\lambda}, y^{-} \eta_{\lambda}\right)=\{x, y\}$ in the q-adic norm.

Proof. Recall from $\S 2.3$ a defining property of $\{-,-\}$ is that

$$
\left\{\theta_{i}, \theta_{i}\right\}\{x, \bar{i} r(y)\}=\left\{\theta_{i} x, \theta_{i} y\right\}
$$

We have $\left(\pi_{i} q_{i}^{2}\right)^{\left\langle i, \lambda+|y|^{\prime}+i^{\prime}\right\rangle} \rightarrow 0$ in the $q$-adic norm as $\lambda \mapsto+\infty$; so using the computations in the proof of Proposition 4.34, we find that

$$
\lim _{\lambda \mapsto \infty}\left(x \eta_{\lambda}, y \eta_{\lambda}\right)=\frac{1}{1-\pi_{i} q_{i}^{2}}\left\{x^{\prime}, E_{i}^{\prime}(y)\right\}=\left\{\theta_{i}, \theta_{i}\right\}\left\{x^{\prime},{ }_{i} r(y)\right\}=\{x, y\}
$$

We note finally the following result, which follows from the $\varrho$-invariance of $(-,-)$ on $\mathbf{f}$.

Proposition 4.36. For $x, y \in \mathbf{f}$, we have

$$
(\varrho(x), \varrho(y))_{P}=(x, y)_{P}
$$

The fact that $\varrho\left(\left.\mathcal{L}\right|_{\pi=1}\right)=\left.\mathcal{L}\right|_{\pi=1}$ follows easily from (4.19) and the orthonormality characterization of crystal lattice in the standard quantum group setting [Kas1]. While such orthonormality characterization fails in general as noted in Remark 4.32(2), the $\varrho$-stable property of the crystal lattice remains to be true. This will require us to use the twistor isomorphism defined in $§ 2.5$.

\subsection{Twistors and the crystal structure}

We let $\mathcal{A}[\mathbf{t}]=\mathbb{Q}(\mathbf{t}) \otimes_{\mathbb{Q}} \mathcal{A}$, the subring of $\mathbb{Q}(q, \mathbf{t})^{\pi}$ of rational functions with no poles at $q=0$, and set $\mathcal{L}[\mathbf{t}]=\mathcal{A}[\mathbf{t}] \otimes_{\mathcal{A}} \mathcal{L} \subset \mathbf{f}[\mathbf{t}]$. The isomorphism $\mathfrak{X}$ from $\S 2.5$, which sends $q \mapsto \mathbf{t}^{-1} q$ and $\pi \mapsto-\pi$, clearly preserves the $\mathbb{Q}(\mathbf{t})$-algebra $\mathcal{A}[\mathbf{t}]$.

Lemma 4.37. The following properties hold: 
1. $\mathfrak{X}\left(\theta_{i}^{(n)}\right)=\theta_{i}^{(n)}$ for $n \geq 1$;

2. ${ }_{i}^{r}(\mathfrak{X}(x))=\mathbf{t}^{\phi(i,|x|-i)} \mathfrak{X}(\bar{i} r(x))$ for all homogeneous $x \in \mathbf{f}[\mathbf{t}]$ and $i \in I$;

3. Let $x \in \mathbf{f}[\mathbf{t}]_{\nu}$ with its $i$-string decomposition $x=\sum_{n \geq 0} \theta_{i}^{(n)} x_{n}$ with $\overline{{ }_{i} r}\left(x_{n}\right)=0$ for a given $i \in I$. Then $\mathfrak{X}(x)$ has the following $i$-string decomposition

$$
\mathfrak{X}(x)=\sum_{n \geq 0} \mathbf{t}^{\phi(n i, \nu)-n^{2} d_{i}} \theta_{i}^{(n)} \mathfrak{X}\left(x_{n}\right) .
$$

Proof. First note we have

$$
\mathfrak{X}\left([n]_{q_{i}, \pi_{i}}\right)=[n]_{\mathbf{t}_{i}^{-1} q_{i},(-1)^{p(i)} \pi_{i}}=\mathbf{t}_{i}^{n-1}[n]_{q_{i}, \pi_{i}}, \quad \theta_{i} * \theta_{i}^{n-1}=\mathbf{t}_{i}^{n-1} \theta_{i}^{n}
$$

We prove (1) by induction on $n$. The case when $n=1$ is clear. Assume $\mathfrak{X}\left(\theta_{i}^{(n-1)}\right)=$ $\theta_{i}^{(n-1)}$. By definition of the divided power, we have

$$
\begin{aligned}
\mathfrak{X}\left(\theta_{i}^{(n)}\right) & =\mathfrak{X}\left([n]_{q_{i}, \pi_{i}}^{-1} \theta_{i} \theta_{i}^{(n-1)}\right) \\
& =\mathbf{t}_{i}^{1-n}[n]_{q_{i}, \pi_{i}}^{-1} \mathfrak{X}\left(\theta_{i}\right) * \mathfrak{X}\left(\theta_{i}^{(n-1)}\right) \\
& =\mathbf{t}_{i}^{1-n}[n]_{q_{i}, \pi_{i}}^{-1} \mathbf{t}_{i}^{n-1} \theta_{i} \theta_{i}^{(n-1)}=\theta_{i}^{(n)} .
\end{aligned}
$$

Next, (2) is immediate from Lemma 2.24 and Proposition 2.22. Finally, we prove (3). Such an identity for $\mathfrak{X}(x)$ follows by the definition of $\mathfrak{X}$ and (1), and the claim that this is an $i$-string decomposition follows from (2).

Proposition 4.38. The isomorphism $\mathfrak{X}$ preserves the lattice $\mathcal{L}[\mathbf{t}]$, i.e., $\mathfrak{X}(\mathcal{L}[\mathbf{t}])=$ $\mathcal{L}[\mathbf{t}]$. Furthermore, $\mathfrak{X}$ induces an isomorphism $\mathfrak{X}_{0}$ on $\mathcal{L}[\mathbf{t}] / q \mathcal{L}[\mathbf{t}]$ such that

$$
\mathfrak{X}_{0}(x)=\mathbf{t}^{\ell(x)} x \quad \forall x \in \mathcal{B},
$$

where $\ell(x)$ is some integer depending on $x$.

Proof. We first observe that $\mathfrak{X}(\mathcal{L}[\mathbf{t}]) \subseteq \mathcal{L}[\mathbf{t}]$, as this follows from using induction on height along with Theorem 4.27(1) and (3), and Lemma 4.37(3). On the other hand, Lemma 4.37 can be rewritten in terms of $\mathfrak{X}^{-1}$ (essentially by replacing $\mathbf{t}$ with 
$\mathbf{t}^{-1}$ in (2) and (3)) and so a similar argument shows $\mathfrak{X}^{-1}(\mathcal{L}[\mathbf{t}]) \subseteq \mathcal{L}[\mathbf{t}]$. Therefore $\mathfrak{X}(\mathcal{L}[\mathbf{t}])=\mathcal{L}[\mathbf{t}]$.

Let $x+q \mathcal{L}[\mathbf{t}] \in \mathcal{B}$. We proceed by induction on the height of $x$. First note that $\mathfrak{X}_{0}(1+q \mathcal{L}[\mathbf{t}])=1+q \mathcal{L}[\mathbf{t}]$ and $\mathfrak{X}_{0}(\pi+q \mathcal{L}[\mathbf{t}])=-\pi+q \mathcal{L}[\mathbf{t}]$, so the proposition holds with $\ell(1+q \mathcal{L}[\mathbf{t}])=0$ and $\ell(\pi+q \mathcal{L}[\mathbf{t}])=2$.

If ht $|x| \geq 1$, then by Theorem $4.27(2)$ and (3), there is an $i \in I$ such that we can write $x+q \mathcal{L}[\mathbf{t}]=\theta_{i}^{(n)} x_{n}+q \mathcal{L}[\mathbf{t}]$ with $x_{n}+q \mathcal{L}[\mathbf{t}] \in \mathcal{B}$ and $n>0$. Then by induction on the height and Lemma 4.37(3), we have

$$
\mathfrak{X}_{0}(x+q \mathcal{L}[\mathbf{t}])=\mathbf{t}^{\phi(n i, \nu)-n^{2} d_{i}+\ell\left(x_{n}+q \mathcal{L}[\mathbf{t}]^{\pi}\right)} x+q \mathcal{L}[\mathbf{t}]
$$

The proposition is proved.

Now we can prove the $\varrho$ invariance of the crystal lattice.

Proposition 4.39. The involution $\varrho$ preserves $\mathcal{L}$, i.e., $\varrho(\mathcal{L})=\mathcal{L}$.

Proof. Since $\frac{1}{2} \in \mathcal{A}$, we note that

$$
\mathcal{L}=\left.\left.\varepsilon_{+} \mathcal{L} \oplus \varepsilon_{-} \mathcal{L} \cong \mathcal{L}\right|_{\pi=1} \oplus \mathcal{L}\right|_{\pi=-1}
$$

We similarly have a decomposition $\varrho=\varrho_{+} \oplus \varrho_{-}$where $\varrho_{ \pm}(x)=\varrho\left(\varepsilon_{ \pm} x\right)$, and by definition we see that under the isomorphism $\left.\varepsilon_{ \pm} \mathbf{f}[\mathbf{t}] \cong \mathbf{f}[\mathbf{t}]\right|_{\pi= \pm 1}, \varrho_{ \pm}$corresponds to $\left.\varrho\right|_{\pi= \pm 1}$.

Since it is known $[\operatorname{Kas} 1, \mathrm{Lu} 4]$ that $\varrho_{\pi=1}\left(\left.\mathcal{L}\right|_{\pi=1}\right)=\left.\mathcal{L}\right|_{\pi=1}$, it suffices to show that

$$
\left.\varrho\right|_{\pi=-1}\left(\left.\mathcal{L}\right|_{\pi=-1}\right)=\left.\mathcal{L}\right|_{\pi=-1}
$$

Since $\mathfrak{X}(\pi)=-\pi$, we have $\mathfrak{X}\left(\left.\mathcal{L}[\mathbf{t}]\right|_{\pi=1}\right)=\left.\mathcal{L}[\mathbf{t}]\right|_{\pi=-1}$. Let $\left.x \in \mathcal{L}\right|_{\pi=-1}$. Since $\left.\left.x \in \mathcal{L}\right|_{\pi=-1} \subset \mathcal{L}[\mathbf{t}]\right|_{\pi=-1}$, by Proposition 2.23 we have

$$
\left.\varrho\right|_{\pi=-1}(x)=\left.\left.(-1)^{\frac{\mathbf{N}(|x|)}{2}+\mathbf{p}(|x|)} \mathfrak{X} \varrho\right|_{\pi=1} \mathfrak{X}^{-1}(x) \in \mathcal{L}[\mathbf{t}]\right|_{\pi=-1} .
$$

On the other hand, by definition we have $\left.\varrho(x) \in \mathbf{f}\right|_{\pi=-1}$, and hence

$$
\left.\left.\left.\varrho\right|_{\pi=-1}(x) \in \mathcal{L}[\mathbf{t}]\right|_{\pi=-1} \cap \mathbf{f}\right|_{\pi=-1}=\left.\mathcal{L}\right|_{\pi=-1}
$$


The proposition is proved.

\subsection{Canonical bases}

Recall the integral form ${ }_{\mathbb{A}} \mathbf{f}$ of $\mathbf{f}$. It is easy to see that ${ }_{\mathbb{A}} \mathbf{f}$ is stable under ${ }^{-}$and ${ }_{i} r$ whence ${ }_{\mathbb{A}} \mathbf{f}$ is stable under Kashiwara operators $\tilde{e}_{i}$ and $\tilde{f}_{i}$; therefore

$$
x=\sum_{n \geq 0} \theta_{i}^{(n)} x_{n} \in{ }_{\mathbb{A}} \mathbf{f} \text { and } e_{i}^{\prime} x_{n}=0 \Longrightarrow x_{n} \in \in_{\mathbb{A}} \mathbf{f}^{-} .
$$

Let ${ }_{\mathbb{A}}\left(\theta_{i}^{n} \mathbf{f}\right)=\theta_{i}^{n} \mathbf{f} \cap_{\mathbb{A}} \mathbf{f}$. Then

$$
\mathbb{A}_{\mathbb{A}}\left(\theta_{i}^{n} \mathbf{f}\right)=\sum_{k \geq n} \theta_{i}^{(k)}{ }_{\mathbb{A}} \mathbf{f}, \quad \text { for } n \geq 0
$$

Moreover, $x=\sum \theta_{i}^{(k)} x_{k} \in{ }_{\mathbb{A}}\left(\theta_{i}^{n} \mathbf{f}\right)$ if and only if $u_{k}=0$ for $k<n$. Set ${ }_{\mathbb{A}} \mathcal{L}=\mathcal{L} \cap_{\mathbb{A}} \mathbf{f}$.

Then ${ }_{\mathbb{A}} \mathcal{L}$ is stable under the Kashiwara operators $\tilde{e}_{i}$ and $\tilde{f}_{i}$. Therefore, $\mathcal{B} \subset{ }_{\mathbb{A}} \mathcal{L} / q_{\mathbb{A}} \mathcal{L} \subset$ $\mathcal{L} / q \mathcal{L}$.

Let $\mathcal{A}_{\mathbb{Z}}$ be the $\mathbb{Z}^{\pi}$-subalgebra of $\mathbb{Q}(q)^{\pi}$ generated by $q$ and $\left(1-\left(\pi q^{2}\right)^{n}\right)^{-1}$ for $n \geq 1$. Letting $K_{\mathbb{Z}}$ be the subalgebra generated by $\mathcal{A}_{\mathbb{Z}}$ and $q^{-1}$, we have $\mathcal{A}_{\mathbb{Z}}=\mathcal{A} \cap K_{\mathbb{Z}}$. Then we see that $\left({ }_{\mathbb{A}} \mathbf{f},{ }_{\mathbb{A}} \mathbf{f}\right)_{P} \subset K_{\mathbb{Z}}$, whence $\left({ }_{\mathbb{A}} \mathcal{L},{ }_{\mathbb{A}} \mathcal{L}\right)_{P} \subset \mathcal{A}_{\mathbb{Z}}$. Therefore, $(-,-)_{P, 0}$ is $\mathbb{Z}^{\pi}$ valued on ${ }_{\mathbb{A}} \mathcal{L} / q_{\mathbb{A}} \mathcal{L}$, and ${ }_{\mathbb{A}} \mathcal{L} / q_{\mathbb{A}} \mathcal{L}$ is a free $\mathbb{Z}^{\pi}$-module with $\pi$-basis $\mathcal{B}$.

Recall $\mathbb{A}_{\mathbb{A}} V(\lambda)={ }_{\mathbb{A}} \mathbf{f} \eta_{\lambda}$. Then ${ }_{\mathbb{A}} V(\lambda)$ is a $\mathbb{A}_{\mathbb{A}} \mathbf{f}$-module. We set also, for $n \geq 0$,

$$
{ }_{\mathbb{A}}\left(F_{i}^{n} V(\lambda)\right)={ }_{\mathbb{A}}\left(\theta_{i}^{n} \mathbf{f}\right)^{-} \eta_{\lambda}=\sum_{k \geq n} F_{i}^{(k)}{ }_{\mathbb{A}} V(\lambda) .
$$

Note that ${ }_{\mathbb{A}} V(\lambda)$ and $\mathbb{A}_{\mathbb{A}}\left(F_{i}^{n} V(\lambda)\right)$ are bar-invariant.

Let ${ }_{\mathbb{A}} \mathcal{L}(\lambda)={ }_{\mathbb{A}} V(\lambda) \cap \mathcal{L}(\lambda)$. Since $\mathcal{L}(\lambda)=\wp_{\lambda}(\mathcal{L})$ we have

$$
{ }_{\mathbb{A}} \mathcal{L}(\lambda) \subset \wp_{\lambda}\left({ }_{\mathbb{A}} \mathcal{L}\right)
$$

and so $\mathcal{B}(\lambda) \subset{ }_{\mathbb{A}} \mathcal{L}(\lambda) / q_{\mathbb{A}} \mathcal{L}(\lambda) \subset \mathcal{L}(\lambda) / q \mathcal{L}(\lambda)$.

The following is a $\pi$-analogue of [Kas1, Lemma 6.1.14]. 
Lemma 4.40. Let $\lambda \in P, i \in I$, and $u \in M^{\lambda}$ for an integrable $\mathbf{U}$-module $M$. Assume $n=-\left\langle\alpha_{i}^{\vee}, \lambda\right\rangle \geq 1$. Then we have

$$
u=\pi_{i}^{\left(\begin{array}{c}
n \\
2
\end{array}\right)} \sum_{k \geq n}(-1)^{k-n}\left[\begin{array}{l}
k-1 \\
k-n
\end{array}\right]_{q_{i}, \pi_{i}} F_{i}^{(k)} E_{i}^{(k)} u .
$$

Proof. We may assume $u=F_{i}^{(m)} v$ with $v \in \operatorname{ker} e_{i} \cap M_{\lambda+m \alpha_{i}}$ with $m \geq n$. Then

$$
\begin{aligned}
\sum_{k \geq n}(-1)^{k-n} & {\left[\begin{array}{l}
k-1 \\
k-n
\end{array}\right]_{q_{i}, \pi_{i}} F_{i}^{(k)} E_{i}^{(k)} u } \\
& =\sum_{k \geq n}(-1)^{k-n}\left[\begin{array}{l}
k-1 \\
k-n
\end{array}\right]_{q_{i}, \pi_{i}} F_{i}^{(k)} E_{i}^{(k)} F_{i}^{(m)} v \\
& =\sum_{m \geq k \geq n} \pi_{i}^{k m+\left(\begin{array}{c}
k+1 \\
2
\end{array}\right)}(-1)^{k-n}\left[\begin{array}{c}
k-1 \\
k-n
\end{array}\right]_{q_{i}, \pi_{i}}\left[\begin{array}{c}
k+m-n \\
k
\end{array}\right]_{q_{i}, \pi_{i}} F_{i}^{(k)} F_{i}^{(m-k)} v \\
& =\sum_{m \geq k \geq n} \pi_{i}^{k m+\left(\begin{array}{c}
k+1 \\
2
\end{array}\right)}(-1)^{k-n}\left[\begin{array}{c}
k-1 \\
k-n
\end{array}\right]_{q_{i}, \pi_{i}}\left[\begin{array}{c}
k+m-n \\
k
\end{array}\right]_{q_{i}, \pi_{i}}\left[\begin{array}{c}
m \\
k
\end{array}\right]_{q_{i}, \pi_{i}} \cdot F_{i}^{(m)} v
\end{aligned}
$$

By a change of variables with $t=k-n$ and $r=m-n$, we have

$$
\begin{aligned}
& \sum_{m \geq k \geq n} \pi_{i}^{k m+\left(\begin{array}{c}
k+1 \\
2
\end{array}\right)}(-1)^{k-n}\left[\begin{array}{c}
k-1 \\
k-n
\end{array}\right]_{q_{i}, \pi_{i}}\left[\begin{array}{c}
k+m-n \\
k
\end{array}\right]_{q_{i}, \pi_{i}}\left[\begin{array}{c}
m \\
k
\end{array}\right]_{q_{i}, \pi_{i}} \\
& =\sum_{t=0}^{r}(-1)^{t} \pi_{i}^{(t+n)(r+n)+\left(\begin{array}{c}
t+n+1 \\
2
\end{array}\right)}\left[\begin{array}{c}
t+n-1 \\
t
\end{array}\right]_{q_{i}, \pi_{i}}\left[\begin{array}{c}
t+r+n \\
r
\end{array}\right]_{q_{i}, \pi_{i}}\left[\begin{array}{c}
r+n \\
t+n
\end{array}\right]_{q_{i}, \pi_{i}} .
\end{aligned}
$$

The proof is completed by applying (2.12).

Proposition 4.41. Let $M \in \mathcal{O}_{\text {int }}$ and ${ }_{\mathbb{A}} M a_{\mathbb{A}} \mathbf{U}$-weight submodule of $M$. Let $\lambda \in X$ and $i \in I$. Suppose $n=-\langle i, \lambda\rangle \geq 0$. Then

$$
{ }_{\mathbb{A}} M_{\lambda}=\sum_{k \geq n} F_{i}^{(k)}{ }_{\mathbb{A}} M_{\lambda+k i^{\prime}}
$$

Proof. The lemma implies that ${ }_{\mathbb{A}} M_{\lambda} \subseteq \sum_{k \geq n} F_{i}^{(k)}{ }_{\mathbb{A}} M_{\lambda+i^{\prime}}$. The reverse inclusion is clear.

Theorem 4.42. Let us consider the following collection $\left(G_{l}\right)$ of statements for $l \geq 0$. 
$\left(G_{l} .1\right)$ For any $\zeta \in \Lambda(l)$,

$$
{ }_{\mathbb{A}} \mathbf{f}_{\zeta} \cap{ }_{\mathbb{A}} \mathcal{L} \cap \overline{\mathbb{A}} \mathcal{L} \rightarrow{ }_{\mathbb{A}} \mathcal{L}_{\zeta} / q_{\mathbb{A}} \mathcal{L}_{\zeta}
$$

is an isomorphism.

$\left(G_{l} .2\right)$ For any $\zeta \in \Lambda(l)$,

$$
{ }_{\mathbb{A}} V(\lambda)_{\lambda+\zeta^{\prime}} \cap_{\mathbb{A}} \mathcal{L}(\lambda) \cap \overline{{ }_{\mathbb{A}} \mathcal{L}(\lambda)} \rightarrow_{\mathbb{A}} \mathcal{L}(\lambda)_{\lambda+\zeta^{\prime}} / q_{\mathbb{A}} \mathcal{L}(\lambda)_{\lambda+\zeta^{\prime}}
$$

is an isomorphism.

Let $G, G_{\lambda}$ be the inverses of these isomorphisms.

$\left(G_{l} .3\right)$ For any $\zeta \in \Lambda(l), n \geq 0$, and $b \in \tilde{f}_{i}^{n}\left(\mathcal{B}_{\zeta+n i}\right)$,

$$
G(b) \in f_{i}^{n} \mathbf{f} .
$$

Then $\left(G_{l}\right)$ holds for each $l \geq 0$.

The rest of this chapter is dedicated to the proof of this theorem. Note that when $l=0$, these statements are obvious. We shall prove $G_{l}$ by induction on $l$, so assume $l>0$ and $G_{l-1}$ holds.

Lemma 4.43. For $\zeta \in \Lambda(l-1)$ we have

$$
\begin{aligned}
\mathbf{A}_{\zeta} \cap{ }_{\mathbb{A}} \mathcal{L} & =\bigoplus_{b \in \mathcal{B}_{\zeta}} \mathbb{Z}^{\pi}[q] G(b), \\
\mathbf{f}_{\zeta} & =\bigoplus_{b \in \mathcal{B}_{\zeta}} \mathbb{Z}^{\pi}\left[q, q^{-1}\right] G(b), \\
{ }_{\mathbb{A}} V(\lambda)_{\lambda+\zeta^{\prime}} \cap \mathcal{L}(\lambda) & =\bigoplus_{b \in \mathcal{B}(\lambda)_{\lambda+\zeta^{\prime}}} \mathbb{Z}^{\pi}[q] G_{\lambda}(b), \\
{ }_{\mathbb{A}} V(\lambda)_{\lambda+\zeta^{\prime}} & =\bigoplus_{b \in \mathcal{B}(\lambda)_{\lambda+\zeta^{\prime}}} \mathbb{Z}^{\pi}\left[q, q^{-1}\right] G_{\lambda}(b) .
\end{aligned}
$$

Proof. This follows from [Kas1, Lemma 7.1.1] applied to the $\pi= \pm 1$ cases and $\left(G_{l-1} \cdot 1\right)-\left(G_{l-1} \cdot 2\right)$. 
Lemma 4.44. For $\zeta \in \Lambda(l-1), b \in{ }_{\mathbb{A}} \mathcal{L} / q_{\mathbb{A}} \mathcal{L}$, and $\lambda \in P^{+}$,

$$
G(b)^{-} \eta_{\lambda}=G_{\lambda}\left(\hat{\wp}_{\lambda} b\right)
$$

Lemma 4.45. For $\zeta \in \Lambda(l-1)$ and $b \in_{\mathbb{A}} \mathcal{L} / q_{\mathbb{A}} \mathcal{L}$, we have

$$
\overline{G(b)}=G(b)
$$

Proof. Set $Q=\frac{G(b)-\overline{G(b)}}{\pi q-q^{-1}}$. Then $Q \in\left({ }_{\mathbb{A}} \mathbf{f}\right)^{\zeta} \cap q_{\mathbb{A}} \mathcal{L} \cap \overline{\mathbb{A}}$, and hence $Q=0$.

The remaining components of the inductive proof of $\left(G_{l} .1\right)-\left(G_{l} .3\right)$ proceed just as in $[$ Kas1, §7.4-7.5]. We summarize the main theorem on canonical bases.

Theorem 4.46. $1 . \mathbb{B}=\{G(b) \mid b \in \mathcal{B}\}$ forms a bar-invariant $\pi$-basis for $\mathbb{A}_{\mathbb{A}} \mathbf{f}$.

2. For every $\lambda \in P^{+}, G(b) \eta_{\lambda}=G_{\lambda}\left(\hat{\wp}_{\lambda} b\right)$. Moreover, $\mathbb{B}(\lambda)=\left\{G_{\lambda}(b) \mid b \in \mathcal{B}(\lambda)\right\}$ forms a bar-invariant $\pi$-basis for $\mathbb{A}_{\mathbb{A}} V(\lambda)$.

We shall freely identify $\mathbb{B}(\lambda)$ with $\left\{b \in \mathbb{B} \mid b^{-} \eta_{\lambda} \neq 0\right\}$.

Our canonical basis is a $\pi$-basis, but not a genuine basis in general. We do not regard this as a defect of our construction though as this is completely natural from the viewpoint of categorification $([\mathrm{HW}]): \pi$ corresponds to "spin" (i.e., a parity shift functor $\Pi$ ), each (projective) indecomposable module $M$ comes from two "spin states" $\{M, \Pi M\}$, and there is no preferred choice among $M$ and $\Pi M$ a priori.

Example 4.47. Assume that $I_{1}$ contains $i, j$ such that $a_{i j}=a_{j i}=0$. Then $F_{i} F_{j}=$ $\pi F_{j} F_{i}$. Both $F_{i} F_{j}$ and $F_{j} F_{i}$ are canonical basis elements in $\mathbf{U}^{-}$, and there is no preferred choice among the two.

Specializing $\pi=1$ yields the usual canonical basis of Lusztig and Kashiwara, while specializing $\pi=-1$ yields a (signed) canonical basis for the half quantum supergroup. Even though we have established a connection on the level of crystal lattices and crystal bases, it is somewhat surprising to see that $\mathfrak{X}$ allows us to establish a direct and precise link between the canonical bases for the two specializations. Recall $\ell(\cdot)$ 
from Proposition 4.38, which is integer-valued but may not be even-integer-valued in general.

Theorem 4.48. For any $b \in \mathcal{B}$, we have

$$
\mathfrak{X}(G(b))=\mathbf{t}^{\ell(b)} G(b) .
$$

In particular, $\mathfrak{X}\left(\left.G(b)\right|_{\pi=1}\right)$ is proportional to $\left.G(b)\right|_{\pi=-1}$.

Proof. It follows by Lemma 2.22 that $\mathfrak{X}(G(b))$ is bar-invariant. It follows by the definition of the maps and Proposition 4.38 that

$$
\mathfrak{X}(G(b))+q \mathcal{L}[\mathbf{t}]=\mathfrak{X}(b)=\mathbf{t}^{\ell(b)} b .
$$

Therefore, $\mathbf{t}^{-\ell(b)} \mathfrak{X}(G(b))=G(b)$ and thus $\mathfrak{X}\left(\varepsilon_{+} G(b)\right)=\varepsilon_{-} \mathbf{t}^{\ell(b)} G(b)$.

Example 4.49. Let $(I, \cdot)$ be the super Cartan datum associated to osp $(1 \mid 4)$ with $I=\{\mathbf{1}, \mathbf{2}\}$ (where $\mathbf{1}$ is the odd simple root). Then

$$
\begin{gathered}
p(\mathbf{1})=1, \quad p(\mathbf{2})=0 ; \\
\mathbf{1} \cdot \mathbf{1}=2, \quad \mathbf{1} \cdot \mathbf{2}=\mathbf{2} \cdot \mathbf{1}=-2, \quad \mathbf{2} \cdot \mathbf{2}=4 ;
\end{gathered}
$$

Furthermore, we can take a choice of $\phi$ in Definition 2.18 such that

$$
\phi\left(\mathbf{1}, \mathbf{1}^{\prime}\right)=1, \quad \phi\left(\mathbf{1}, \mathbf{2}^{\prime}\right)=0, \quad \phi\left(\mathbf{2}, \mathbf{1}^{\prime}\right)=-2, \quad \phi\left(\mathbf{2}, \mathbf{2}^{\prime}\right)=2
$$

It is an easy computation that

$$
\tilde{f}_{\mathbf{1}} \tilde{f}_{\mathbf{2}} \tilde{f}_{\mathbf{1}} 1=\theta_{\mathbf{1}}\left(\theta_{\mathbf{2}} \theta_{\mathbf{1}}-q^{2} \theta_{\mathbf{1}} \theta_{\mathbf{2}}\right)+q^{2} \theta_{\mathbf{1}}^{(2)} \theta_{\mathbf{2}}
$$

In particular, $G\left(\tilde{f}_{\mathbf{1}} \tilde{f}_{\mathbf{2}} \tilde{f}_{\mathbf{1}} 1+q \mathcal{L}\right)=\theta_{\mathbf{1}} \theta_{\mathbf{2}} \theta_{\mathbf{1}}$, and $\mathfrak{X}\left(G\left(\tilde{f}_{\mathbf{1}} \tilde{f}_{\mathbf{2}} \tilde{f}_{\mathbf{1}} 1+q \mathcal{L}\right)\right)=\mathbf{t}^{-1} \theta_{\mathbf{1}} \theta_{\mathbf{2}} \theta_{\mathbf{1}}$.

On the other hand, $G\left(\tilde{f}_{\mathbf{1}} \tilde{f}_{\mathbf{1}} \tilde{f}_{\mathbf{2}} 1\right)=\theta_{\mathbf{1}}^{(2)} \theta_{\mathbf{2}}$ and $\mathfrak{X}\left(G\left(\tilde{f}_{\mathbf{1}} \tilde{f}_{\mathbf{1}} \tilde{f}_{\mathbf{2}} 1\right)\right)=\theta_{\mathbf{1}}^{(2)} \theta_{\mathbf{2}}$. In particular, note that $\ell(b)$ is not constant on $\mathbb{B}_{\nu}$ for $\nu \in \mathbb{N}[I]$.

Lastly, we note the following additional properties of $\mathbb{B}$. 
Lemma 4.50. Let $(-,-)$ be the polarization on $V(\lambda)$.

1. We have $\left({ }_{\mathbb{A}} V(\lambda),{ }_{\mathbb{A}} V(\lambda)\right) \subset \mathbb{A}$.

2. $\left(b^{-} \eta_{\lambda}, b^{\prime-} \eta_{\lambda}\right) \in \operatorname{sgn}(b) \delta_{b, b^{\prime}}+q \mathbb{Z}[q]^{\pi}$ for any $b, b^{\prime} \in \mathbb{B}$.

3. For $b \in \mathbb{B}$, either $\varrho(b)=b$ or $\varrho(b)=\pi b$, so in particular $\varrho(\mathbb{B})=\mathbb{B}$.

4. $b \eta_{\lambda}=0$ if and only if $b \in{ }_{\mathbb{A}} \mathbf{f} \theta_{i}^{(n)}$ for some $i \in I$ and $n \geq\langle i, \lambda\rangle+1$.

Proof. (1) and (2) are proved analogously to [Lu4, Proposition 19.3.3].

For $(3)$, it is known that $\left.\mathbb{B}\right|_{\pi=1}$ is $\rho$-invariant ([Lu4, Theorem 14.4.3(c)]). By Proposition 2.23, $\varrho \mathfrak{X}$ and $\mathfrak{X} \varrho$ are equal up to a sign. Then $\mathfrak{X}(\varrho(b))=s \mathbf{t}^{\ell(b)} \varrho(b)$, where $s= \pm 1$. But then using $\varrho$-invariance of the canonical basis when $\pi=1$,

$$
\mathbf{t}^{\ell(b)} \epsilon_{-} b=\mathfrak{X}\left(\epsilon_{+} b\right)=\mathfrak{X}\left(\varrho\left(\epsilon_{+} b\right)\right)=\varsigma \mathbf{t}^{\ell(b)} \epsilon_{-} \varrho(b) .
$$

Since $-\epsilon_{-}=\pi \epsilon_{-}, \varrho\left(\epsilon_{-} b\right)=\epsilon_{-} b$ or $\epsilon_{-} \pi b$. Therefore, $\varrho(b)=b$ or $\varrho(b)=\pi b$, as claimed.

Finally, we note that $\mathfrak{X}\left(\mathbf{f}[\mathbf{t}] \theta_{i}^{n}\right) \subset \mathbf{f}[\mathbf{t}] \theta_{i}^{n}$, the statement of (4) holds for $\left.\mathbb{B}\right|_{\pi=1}$ (cf. [Lu4, Theorem 14.4.11]), and we have $\mathfrak{X}\left(\epsilon_{ \pm} b\right)=\mathbf{t}^{\ell(b)} \epsilon_{\mp} b$. Combining these facts proves (4) for $\left.\mathbb{B}\right|_{\pi=-1}$ and hence for $\mathbb{B}$. 


\section{Chapter 5}

\section{The modified form of $U$}

\subsection{The modified form}

Definition 5.1. The modified quantum covering group $\dot{\mathbf{U}}$ associated to the root datum $(Y, X, I, \cdot)$ is the associative $\mathbb{Q}(q)^{\pi}$-algebra without unit on symbols $x 1_{\lambda}$ and $1_{\lambda} x$ for $x \in \mathbf{U}$ and $\lambda \in X$ satisfying the relations

$$
\begin{gathered}
x 1_{\lambda} y 1_{\eta}=\delta_{\lambda, \eta+|y|}(x y) 1_{\lambda}, \quad x 1_{\lambda}=1_{\lambda+|x|} x \quad \text { for all homogeneous } x, y \in \mathbf{U}, \lambda \in X, \\
K_{\nu} 1_{\lambda}=q^{\langle\nu, \lambda\rangle} 1_{\lambda}, \quad J_{\nu} 1_{\lambda}=\pi^{\langle\nu, \lambda\rangle} 1_{\lambda} \quad \text { for all } \nu \in Y, \lambda \in X .
\end{gathered}
$$

Remark 5.2. A version of $\dot{\mathbf{U}}$ was defined in [CFLW, ClW] in different ways. In [CFLW, Definition 4.2], the modified form is defined using generators $1_{\lambda}, E_{i} 1_{\lambda}$ and $F_{i} 1_{\lambda}$ for $i \in I$ and $\lambda \in X$ satisfying certain relations; it is straightforward to see that this is equivalent to our definition. In [ClW, §6.1], a rank one modified form is defined using certain quotients of $\mathbf{U}$ in direct parallel to the definition in [Lu4, §23.1]. This construction can be generalized to higher rank, and results in an algebra isomorphic to our definition.

The algebra $\dot{\mathbf{U}}$ is naturally a $\pi$-free $\mathbf{U}$-bimodule under the following action: for $x, y, z \in \mathbf{U}$, we set

$$
x\left(y 1_{\lambda}\right) z=(x y z) 1_{\lambda-|z|} .
$$

We note the following commutation relations which may be deduced from Lemma 3.7. 
Lemma 5.3. We have the following identities in $\dot{\mathbf{U}}$ :

$$
\begin{aligned}
& E_{i}^{(N)} 1_{\lambda} F_{i}^{(M)}=\sum_{t} \pi_{i}^{M N-\left(\begin{array}{c}
t+1 \\
2
\end{array}\right)} F_{i}^{(M-t)}\left[\begin{array}{c}
M+N+\langle i, \lambda\rangle \\
t
\end{array}\right]_{q_{i}, \pi_{i}} 1_{\lambda+2 N+2 M-2 t} E_{i}^{(N-t)}, \\
& F_{i}^{(N)} 1_{\lambda} E_{i}^{(M)}=\sum_{t}(-1)^{t} \pi_{i}^{(M-t)(N-t)-t^{2}} E_{i}^{(M-t)}\left[\begin{array}{c}
M+N-\langle i, \lambda\rangle \\
t
\end{array}\right]_{q_{i}, \pi_{i}} 1_{\lambda-2 N-2 M+2 t} F_{i}^{(N-t)}, \\
& E_{i}^{(N)} F_{j}^{(M)} 1_{\lambda}=\pi^{M N p(i) p(j)} F_{j}^{(M)} E_{i}^{(N)} 1_{\lambda} \quad \text { if } i \neq j .
\end{aligned}
$$

The next proposition shows that the various algebra (anti)automorphisms on $\mathbf{U}$ we have previously defined have analogous (anti)automorphisms on $\mathbf{U}$ which are compatible with the bimodule presentation.

\section{Proposition 5.4.}

1. There exists a $\mathbb{Q}(q)^{\pi}$-algebra automorphism $\omega$ of $\dot{\mathbf{U}}$ satisfying

$$
\omega\left(x 1_{\lambda}\right)=\omega(x) 1_{-\lambda} .
$$

2. There exists a $\mathbb{Q}(q)^{\pi}$-algebra antiautomorphism @ of $\mathbf{U}$ satisfying

$$
\varrho\left(x 1_{\lambda}\right)=1_{-\lambda} \varrho(x) .
$$

3. There exists a $\mathbb{Q}^{\pi}$-algebra involution - of $\mathbf{U}$ satisfying

$$
\overline{x 1_{\lambda}}=\bar{x} 1_{\lambda}
$$

4. There exists a $\mathbb{Q}^{\pi}$-algebra involution $\dagger$ of $\dot{\mathbf{U}}$ satisfying

$$
\left(x 1_{\lambda}\right)^{\dagger}=x^{\dagger} 1_{\lambda}
$$

5. Let $\dot{\mathbf{U}}[\mathbf{t}]=\mathbb{Q}(q, \mathbf{t})^{\pi} \otimes_{\mathbb{Q}(q)^{\pi}} \dot{\mathbf{U}}$. There is an automorphism $\mathfrak{X}: \dot{\mathbf{U}}[\mathbf{t}] \rightarrow \dot{\mathbf{U}}[\mathbf{t}]$ such that $\mathfrak{X}\left(u 1_{\lambda} u^{\prime}\right)=\mathfrak{X}(u) 1_{\lambda} \mathfrak{X}\left(u^{\prime}\right)$ for $u, u^{\prime} \in \hat{\mathbf{U}}[\mathbf{t}]$.

Proof. This follows as an elementary consequence of the existence of these maps on $\mathbf{U}$ and the $\mathbf{U}$-module structure on $\dot{\mathbf{U}}$. In particular, for $\mathfrak{X}$, we note that there is a natural $\hat{\mathbf{U}}[\mathbf{t}]$ on $\dot{\mathbf{U}}[\mathbf{t}]$ given by extending the $\mathbf{U}$-action via $T_{\nu} \Upsilon_{\mu} 1_{\lambda}=\mathbf{t}^{\langle\nu, \lambda\rangle+\phi(\mu, \lambda)} 1_{\lambda}$. 
From the triangular decomposition of $\mathbf{U}$, $\mathbf{U}$ has a $\pi$-basis consisting of elements of the form $b^{+} J_{\mu} K_{\nu} b^{-}$where $\left(b, b^{\prime}\right) \in \mathbb{B} \times_{\pi} \mathbb{B}$ (where here we are using the notation from (3.28)) and $\mu, \nu \in Y$. Since

$$
\left(b^{+} J_{\mu} K_{\nu} b^{\prime-}\right) 1_{\lambda}=\pi^{\left\langle\mu, \lambda_{1}\right\rangle} q^{\left\langle\nu, \lambda_{1}\right\rangle} b^{+} 1_{\lambda_{1}} b^{\prime-}
$$

where $\lambda_{1}=\lambda-\left|b^{\prime}\right|$, we see that $\left\{b^{+} 1_{\lambda} b^{\prime-}:\left(b, b^{\prime}\right) \in \mathbb{B} \times_{\pi} \mathbb{B}\right\}$ forms a $\pi$-basis of $\dot{\mathbf{U}}$, Similarly, $\left\{b^{-} 1_{\lambda} b^{+}:\left(b, b^{\prime}\right) \in \mathbb{B} \times_{\pi} \mathbb{B}\right\}$ forms a $\pi$-basis of $\dot{\mathbf{U}}$. In fact, these elements span an integral form of $\dot{\mathbf{U}}$.

\section{Lemma 5.5.}

1. The $\mathbb{A}$-submodule of $\dot{\mathbf{U}}$ spanned by the elements $x^{+} 1_{\lambda} x^{\prime-}$ (with $x, x^{\prime} \in{ }_{\mathbb{A}} \mathbf{f}$ ) coincides with the $\mathbb{A}$-submodule of $\dot{\mathbf{U}}$ spanned by the elements $x^{-} 1_{\lambda} x^{+}$(with $\left.x, x^{\prime} \in{ }_{\mathbb{A}} \mathbf{f}\right)$. We denote it by ${ }_{\mathbb{A}} \dot{\mathbf{U}}$.

2. The elements $\left\{b^{+} 1_{\lambda} b^{\prime-}:\left(b, b^{\prime}\right) \in \mathbb{B} \times_{\pi} \mathbb{B}\right\} \quad\left(\right.$ resp. $\left.\quad\left\{b^{-} 1_{\lambda} b^{\prime+}:\left(b, b^{\prime}\right) \in \mathbb{B} \times_{\pi} \mathbb{B}\right\}\right)$ form a $\pi$-basis of ${ }_{\mathbb{A}} \dot{\mathbf{U}}$.

3. ${ }_{\mathbb{A}} \dot{\mathbf{U}}$ is a $\mathbb{A}$-subalgebra of $\dot{\mathbf{U}}$ which is generated by the elements $E_{i}^{(n)} 1_{\lambda}$ and $F_{i}^{(n)} 1_{\lambda}$ for $i \in I, n \geq 0$, and $\lambda \in X$.

Proof. Recall that ${ }_{\mathbb{A}} \mathbf{f}$ is the $\mathbb{Z}\left[q, q^{-1}\right]^{\pi}$ generated by $\theta_{i}^{(n)}$ for $i \in I$ and $n \geq 0$, and so repeated application of Lemma 5.3 implies (1) and (3). (2) follows from the triangular decomposition of $\dot{\mathbf{U}}$.

Now let us examine the connection between the representation theory of $\mathbf{U}$ and $\dot{\mathbf{U}}$. We have the following natural family of $\dot{\mathbf{U}}$-modules.

Definition 5.6. $A$ Uं-module $M$ is called unital if for any $m \in M, m=\bigoplus_{\lambda \in X} 1_{\lambda} m$ with $1_{\lambda} m=0$ for all but finitely many $\lambda \in X$.

Unital Ü-modules may naturally be viewed as weight $\mathbf{U}$-modules by interpreting the idempotent $1_{\lambda}$ as a projection onto the $\lambda$-weight space. More precisely, any 
unital $\dot{\mathbf{U}}$-module $M$ is a weight $\mathbf{U}$-module under the action $x \cdot m=\sum_{\lambda \in X} x 1_{\lambda} m$, and similarly any weight $\mathbf{U}$-module $M$ is a unital $\dot{\mathbf{U}}$-module via $x 1_{\lambda} m=x m_{\lambda}$, where $m_{\lambda}$ is the orthogonal projection of $m$ to the $M_{\lambda}$ weight space.

The algebra $\dot{U}$ does not admit a natural co-product, as any candidate would require an infinite sum (cf. [Lu4, §23.1.5]). However, since unital modules and weight modules are equivalent, the Hopf structure on $\mathbf{U}$ imposes a unital $\mathbf{U}$-module structure on the tensor product of weight modules.

In fact, the structure of $\dot{\mathbf{U}}$ is intimately related to tensor products of modules. Recall that the Verma module $M(\lambda)$ is isomorphic to $\mathbf{f}$ as a free $\mathbb{Q}(q)^{\pi}$-module. We shall now describe a generalization of this identification to $\dot{\mathbf{U}}$.

Proposition 5.7. Let $\lambda, \lambda^{\prime} \in X$. The $\mathbb{Q}(q)^{\pi}$-linear map

$$
\partial_{\lambda, \lambda^{\prime}}: \dot{\mathbf{U}} 1_{\lambda-\lambda^{\prime}} \rightarrow M(\lambda) \otimes{ }^{\omega} M\left(\lambda^{\prime}\right), \quad u \mapsto u(1 \otimes 1)
$$

is an isomorphism. Similarly, the $\mathbb{A}$-linear map

$$
{ }_{\mathbb{A}} \partial_{\lambda, \lambda^{\prime}}: \dot{\mathbb{U}} 1_{\lambda-\lambda^{\prime}} \rightarrow{ }_{\mathbb{A}} M(\lambda) \otimes_{\mathbb{A}}^{\omega}{ }_{\mathbb{A}}^{\omega} M\left(\lambda^{\prime}\right), \quad u \mapsto u(1 \otimes 1)
$$

is an isomorphism.

Proof. The U-module $M(\lambda) \otimes{ }^{\omega} M\left(\lambda^{\prime}\right)$ is naturally a weight U-module, hence a $\dot{\mathbf{U}}$ module. As it will often be convenient, we will use the $\mathbb{Q}(q)^{\pi}$-module identification

$$
M(\lambda) \otimes{ }^{\omega} M\left(\lambda^{\prime}\right)=\mathbf{f} \otimes \mathbf{f} .
$$

From the triangular decomposition of $\dot{\mathbf{U}}$, the elements $b^{+} b^{\prime-} 1_{\zeta}$ with $\left(b, b^{\prime}\right) \in \mathbb{B} \times_{\pi} \mathbb{B}$ and $\zeta \in X$ comprise a $\pi$-basis of $\dot{\mathbf{U}}$. Likewise, the canonical basis of $\mathbf{f}$ induces a $\pi$-basis $\mathbb{B} \otimes \mathbb{B}=\left\{b \otimes b^{\prime} \mid\left(b, b^{\prime}\right) \in \mathbb{B} \times_{\pi} \mathbb{B}\right\}$ on $\mathbf{f} \otimes \mathbf{f}$.

If $\zeta=\lambda-\lambda^{\prime}$, then since a $\pi$-basis over $\mathbb{Q}(q)^{\pi}$ is in particular a $\mathbb{Q}(q)$-basis, we have

$$
b^{+} b^{\prime-} 1_{\zeta}(1 \otimes 1)=b^{+}\left(b^{\prime} \otimes 1\right)=\pi^{p\left(b^{\prime}\right) p(b)} b^{\prime} \otimes b+\sum c_{b_{1}^{\prime}, b_{1}} b_{1}^{\prime} \otimes b_{1}
$$


where $c_{b_{1}^{\prime}, b_{1}} \in \mathbb{Q}(q)$ are constants, $\nu=|b|$, and the sum is over $\left(b_{1}, b_{1}^{\prime}\right) \in \mathbb{B} \times_{\pi} \mathbb{B}$ such that ht $\left|b_{1}\right|<\mathrm{ht}|b|, \mathrm{ht}\left|b_{1}^{\prime}\right|<\mathrm{ht}\left|b^{\prime}\right|$ and $b_{1}^{\prime}$ belongs to the $\dot{\mathbf{U}}_{\text {-submodule of } M(\lambda)}$ generated by $b^{\prime}$. Moreover, since $\mathbb{B} \subset{ }_{\mathbb{A}} \mathbf{U}$, the left-hand side of (5.3) lies in ${ }_{\mathbb{A}} \mathbf{f} \otimes_{\mathbb{A} A} \mathbf{f}$ and hence we have $c_{b_{1}^{\prime}, b_{1}} \in \mathbb{Z}\left[q, q^{-1}\right]$.

Similarly, from the triangular decomposition of $\dot{\mathbf{U}}$, the elements $b^{-} b^{\prime+} 1_{\zeta}$ comprise a $\pi$-basis of $\dot{\mathbf{U}}$. If $\zeta=\lambda-\lambda^{\prime}$, then

$$
b^{-} b^{\prime+} 1_{\zeta}(1 \otimes 1)=b^{-}\left(1 \otimes b^{\prime}\right)=b \otimes b^{\prime}+\sum c_{b_{1}, b_{1}^{\prime}} b_{1} \otimes b_{1}^{\prime},
$$

where $c_{b_{1}, b_{1}^{\prime}}^{\prime} \in \mathbb{Z}\left[q, q^{-1}\right]$ are constants and the sum is over $b_{1}, b_{1}^{\prime} \in \mathbb{B} \times_{\pi} \mathbb{B}$ such that $\mathrm{ht}\left|b_{1}\right|<\mathrm{ht}|b|$, ht $\left|b_{1}^{\prime}\right|<\mathrm{ht}\left|b^{\prime}\right|$ and $b_{1}^{\prime}$ belongs to the $\dot{\mathbf{U}}$-submodule of ${ }^{\omega} M\left(\lambda^{\prime}\right)$ generated by $b^{\prime}$.

In either case, note that the transition matrix between the $\pi$-basis $\mathbb{B} \otimes \mathbb{B}$ and the elements $\left\{b^{-} b^{\prime+} 1_{\zeta}(1 \otimes 1) \mid b, b^{\prime} \in \mathbb{B} \times_{\pi} \mathbb{B}\right\}$ is upper unitriangular with entries in $\mathbb{Z}\left[q, q^{-1}\right]$, hence the latter is also a $\pi$-basis.

Now let us examine the relationship between the modules $N\left(\lambda, \lambda^{\prime}\right)$ from (3.15) and $M(\lambda) \otimes{ }^{\omega} M\left(\lambda^{\prime}\right)$. Let $\zeta \in X$ and let $a=\sum_{i} a_{i} i, a^{\prime}=\sum_{i} a_{i}^{\prime} i \in \mathbb{N}[I]$ such that $\langle i, \zeta\rangle=a_{i}^{\prime}-a_{i}$ for all $i \in I$. We define the ideals

$$
\begin{gathered}
P\left(\zeta, a, a^{\prime}\right)=\sum_{i, n>a_{i}} \dot{\mathbf{U}} F_{i}^{(n)} 1_{\zeta}+\sum_{i, n>a_{i}^{\prime}} \dot{\mathbf{U}} E_{i}^{(n)} 1_{\zeta}, \\
{ }_{\mathbb{A}} P\left(\zeta, a, a^{\prime}\right)=\sum_{i, n>a_{i}} \dot{\mathbf{U}} F_{i}^{(n)} 1_{\zeta}+\sum_{i, n>a_{i}^{\prime}} \dot{\mathbf{U}} E_{i}^{(n)} 1_{\zeta} .
\end{gathered}
$$

Proposition 5.8. Let $\lambda, \lambda^{\prime} \in X^{+}$and set $a_{i}=\langle i, \lambda\rangle, a_{i}^{\prime}=\left\langle i, \lambda^{\prime}\right\rangle$ for all $i \in I$. Set $\zeta=$ $\lambda^{\prime}-\lambda, a=\sum_{i} a_{i} i \in \mathbb{N}[I]$, and $a^{\prime}=\sum_{i} a_{i}^{\prime} \in \mathbb{N}[I]$. The map $u \mapsto u\left(\eta_{\lambda} \otimes \xi_{-\lambda^{\prime}}\right)$ defines a surjective linear map $\dot{\mathbf{U}} \rightarrow N\left(\lambda, \lambda^{\prime}\right)$ with kernel equal to $P\left(\zeta, a, a^{\prime}\right)$. Moreover, restriction of this map to ${ }_{\mathbb{A}} \dot{U}$ gives a surjective linear map ${ }_{\mathbb{A}} \dot{U} \rightarrow{ }_{\mathbb{A}} N\left(\lambda, \lambda^{\prime}\right)$ with kernel equal to ${ }_{\mathbb{A}} P\left(\zeta, a, a^{\prime}\right)$.

Proof. Let $\mathcal{T}$ (resp. $\mathcal{T}^{\prime}$ ) be the kernel of the canonical homomorphism of $\mathbf{U}$-modules $\mathbf{f}=M(\lambda) \rightarrow V(\lambda)\left(\right.$ resp. $\left.\mathbf{f}={ }^{\omega} M\left(\lambda^{\prime}\right) \rightarrow{ }^{\omega} V\left(\lambda^{\prime}\right)\right)$. Then by Theorem 4.46 and Lemma 
4.50 (4), $\mathcal{T}$ (resp. $\mathcal{T}^{\prime}$ ) is generated by $b \in \mathbb{B}$ such that $b \in{ }_{\mathbb{A}} \mathbf{f} \theta_{i}^{(n)}$ for some $n \geq a_{i}$ (resp. $n \geq a_{i}^{\prime}$ ).

Then taking tensor products, we obtain the surjective homomorphism

$$
M(\lambda) \otimes{ }^{\omega} M\left(\lambda^{\prime}\right) \rightarrow N\left(\lambda, \lambda^{\prime}\right)
$$

The kernel of this map is the subspace $\mathcal{T} \otimes \mathbf{f}+\mathbf{f} \otimes \mathcal{T}^{\prime}$.

Let $\mathbb{D}(\lambda)=\mathbb{B} \backslash \mathbb{B}(\lambda)$. Now by the triangular decomposition, the description of $\mathcal{T}$, and (5.3), $\partial_{\lambda, \lambda^{\prime}}$ maps the subspace

$$
\sum_{b \in \mathbb{D}(\lambda), b^{\prime} \in \mathbb{B}} \mathbb{Q}(q)^{\pi} b^{\prime+} b^{-} 1_{\zeta}=\sum_{i, n>a_{i}} \dot{\mathbf{U}} F_{i}^{(n)} 1_{\zeta}
$$

onto the subspace

$$
\sum_{b \in \mathbb{D}(\lambda), b^{\prime} \in \mathbb{B}} \mathbb{Q}(q)^{\pi} b \otimes b^{\prime}=\mathcal{T} \otimes \mathbf{f} .
$$

Similarly by the triangular decomposition, the description of $\mathcal{T}^{\prime}$, and (5.4), $\partial_{\lambda, \lambda^{\prime}}$ maps the subspace

$$
\sum_{b \in \mathbb{B}, b^{\prime} \in \mathbb{D}\left(\lambda^{\prime}\right)} \mathbb{Q}(q)^{\pi} b^{-} b^{\prime+} 1_{\zeta}=\sum_{i, n>a_{i}} \dot{\mathbf{U}} E_{i}^{(n)} 1_{\zeta}
$$

onto the subspace

$$
\sum_{b \in \mathbb{B}, b^{\prime} \in \mathbb{D}\left(\lambda^{\prime}\right)} \mathbb{Q}(q)^{\pi} b \otimes b^{\prime}=\mathbf{f} \otimes \mathcal{T}^{\prime} .
$$

We note that replacing everything with the integral form in the preceding argument does not effect the argument, which finishes the proof.

We now note that each module $N\left(\lambda, \lambda^{\prime}\right)$ comes equipped with a $\pi$-basis. Indeed, the elements

$$
\mathbb{B}(\lambda) \otimes \mathbb{B}\left(\lambda^{\prime}\right)=\left\{b^{+} \xi_{-\lambda} \otimes b^{\prime-} \eta_{\lambda^{\prime}}: b \times b^{\prime} \in \mathbb{B}(\lambda) \times_{\pi} \mathbb{B}\left(\lambda^{\prime}\right)\right\}
$$

form a $\pi$-basis of $N\left(\lambda, \lambda^{\prime}\right)$. They generate a $\mathbb{Z}[q]^{\pi}$-submodule $\mathcal{L}=\mathcal{L}\left(\lambda, \lambda^{\prime}\right)$ and a $\mathbb{A}$-submodule $\mathbb{A} \mathcal{L}$. 


\subsection{A bar involution on $N\left(\lambda, \lambda^{\prime}\right)$}

Recall the quasi-R-matrix $\Theta=\sum_{\nu \in \mathbb{N}[I]} \Theta_{\nu}$ from $\S 3.7$. We recall in particular that $\Theta$ lives in a suitable completion of $\mathbf{U} \otimes \mathbf{U}$, and that (in this completion) we have

$$
\Delta(u) \Theta=\Theta \bar{\Delta}(u), \quad \Theta \bar{\Theta}=1 .
$$

where $^{-}: \mathbf{U} \otimes \mathbf{U} \rightarrow \mathbf{U} \otimes \mathbf{U}$ is the map $\overline{x \otimes y}=\bar{x} \otimes \bar{y}$.

Let $M$ and $M^{\prime}$ be weight modules such that ${ }^{\omega} M \in \mathcal{O}$ or $M^{\prime} \in \mathcal{O}$. Then since $\Theta_{\nu} \in \mathbf{U}_{\nu}^{+} \otimes \mathbf{U}^{-}$, given any $x \in M \otimes M^{\prime}$, we must have $\Theta_{\nu} x=0$ for all but a finite number of $\nu \in \mathbb{N}[I]$. Then by regarding $M \otimes M^{\prime}$ as a $\mathbf{U} \otimes \mathbf{U}$-module, $\Theta$ defines a linear map $\Theta: M \otimes M^{\prime} \rightarrow M \otimes M^{\prime}$ by $m \otimes m^{\prime} \mapsto \sum \Theta_{\nu}\left(m \otimes m^{\prime}\right)$. This is well-defined because only finitely many terms may be non-zero, and we see that

$$
\Delta(u) \Theta\left(m \otimes m^{\prime}\right)=\Theta\left(\overline{\Delta(\bar{u})} m \otimes m^{\prime}\right) .
$$

In particular, suppose that $M$ and $M^{\prime}$ are equipped with bar-involutions ${ }^{-}: M \rightarrow M$ and ${ }^{-}: M^{\prime} \rightarrow M^{\prime}$ such that $\overline{u m}=\overline{u m}$ and $\overline{u m^{\prime}}=\overline{u m^{\prime}}$ for all $u \in \mathbf{U}, m \in M$ and $m^{\prime} \in M^{\prime}$. Then

$$
\Delta(u) \Theta\left(m \otimes m^{\prime}\right)=\Theta\left(\overline{\Delta(\bar{u}) \bar{m} \otimes \bar{m}^{\prime}}\right),
$$

where ${ }^{-}={ }^{-} \otimes^{-}: M \otimes M^{\prime} \rightarrow M \otimes M^{\prime}$.

Theorem 5.9. Let $\lambda, \lambda^{\prime} \in X$ and consider the Verma modules $M(\lambda)$ and $M\left(\lambda^{\prime}\right)$. Set $M=M(\lambda) \in \mathcal{O}$ and $M^{\prime}={ }^{\omega} M\left(\lambda^{\prime}\right) \in \mathcal{C}$. Then $\Theta$ is a well defined map on $M \otimes M^{\prime}$ which leaves stable the $\mathbb{A}$-submodule ${ }_{\mathbb{A}} M \otimes_{\mathbb{A}}\left({ }_{\mathbb{A}}^{\omega} M^{\prime}\right)$.

Proof. The proof of this result is essentially the same as in [Lu4, Prop 24.1.4], but we shall state it here for completeness.

Since the ambient space of $M(\lambda)$ and $M\left(\lambda^{\prime}\right)$ is $\mathbf{f}$, there are well defined maps ${ }^{-}: M \rightarrow M$ and ${ }^{-}: M^{\prime} \rightarrow M^{\prime}$. On the other hand, $M(\lambda)$ and ${ }^{\omega} M\left(\lambda^{\prime}\right)$ may be identified as $\mathbf{U}$-modules with certain quotients of $\mathbf{U}$ such that the bar involution on 
$\mathbf{U}$ induces those on $M$ and $M^{\prime}$, whence $\overline{u m}=\overline{u m}$ and $\overline{u m^{\prime}}=\bar{u} \overline{m^{\prime}}$ for $u \in \mathbf{U}, m \in M$ and $m^{\prime} \in M^{\prime}$. Then we set ${ }^{-}={ }^{-} \otimes^{-}: M \otimes M^{\prime} \rightarrow M \otimes M^{\prime}$.

Now let us identify $M(\lambda)$ and ${ }^{\omega} M\left(\lambda^{\prime}\right)$ with $\mathbf{f}$. We note that by definition, $\overline{1}=1$ in $M$ and $M^{\prime}$. Moreover, $\Theta(1 \otimes 1)=1 \otimes 1$. Then we have

$$
u(1 \otimes 1)=\Theta(\overline{\bar{u}(1 \otimes 1)})
$$

Since the ambient space of $\mathbb{A}_{\mathbb{A}} M(\lambda)$ and ${ }_{\mathbb{A}}^{\omega} M\left(\lambda^{\prime}\right)$ is ${ }_{\mathbb{A}} \mathbf{f}$, which is bar-invariant, we see that ${ }_{\mathbb{A}} M(\lambda) \otimes_{\mathbb{A}}\left({ }_{\mathbb{A}}^{\omega} M\left(\lambda^{\prime}\right)\right)$ is stable under - . Take $x \in{ }_{\mathbb{A}} M(\lambda) \otimes_{\mathbb{A}}\left({ }_{\mathbb{A}}^{\omega} M\left(\lambda^{\prime}\right)\right)$, and set $x^{\prime}=\bar{x} \in \mathbb{A} M(\lambda) \otimes_{\mathbb{A}}\left(\underset{\mathbb{A}}{\omega} M\left(\lambda^{\prime}\right)\right)$.

On the other hand, the isomorphism ${ }_{\mathbb{A}} \dot{\mathbf{U}} 1_{\lambda^{\prime}-\lambda} \rightarrow{ }_{\mathbb{A}} M(\lambda) \otimes_{\mathbb{A}}\left({ }_{\mathbb{A}}^{\omega} M\left(\lambda^{\prime}\right)\right)$ implies there is a $u^{\prime} \in{ }_{\mathbb{A}} \dot{\mathbf{U}} 1_{\lambda-\lambda^{\prime}}$ such that $u^{\prime}(1 \otimes 1)=x^{\prime}$. There is also a $u=\overline{u^{\prime}} \in{ }_{\mathbb{A}} \dot{\mathbf{U}} 1_{\lambda-\lambda^{\prime}}$. Therefore, $x=\overline{x^{\prime}}=\overline{u^{\prime}(1 \otimes 1)}=\overline{\bar{u}(1 \otimes 1)}$, and so $\Theta(x)=u(1 \otimes 1) \in_{\mathbb{A}} M(\lambda) \otimes_{\mathbb{A}}\left({ }_{\mathbb{A}}^{\omega} M\left(\lambda^{\prime}\right)\right)$.

Remark 5.10. In fact, it can be shown that the conclusion of the theorem holds for any "sufficiently nice" choice of module $M^{\prime}$; that is, one possessing a basis analogous to $\mathbf{B}, \mathbf{B}(\lambda)$. (These are what Lusztig calls "based modules", (cf. [Lu4, §27] which we do not develop here.) This was recently observed by Bao and Wang [BW] in the Drinfeld-Jimbo quantum group setting; the proof amounts to observing that the module is "approximately cyclic" and generalizing the above proof. We note that the proof their transfers over almost word for word to the covering quantum group setting.

This immediately implies the following corollary.

Corollary 5.11. The map $\Theta$ leaves stable the $\mathbb{A}$-submodule ${ }_{\mathbb{A}} N\left(\lambda, \lambda^{\prime}\right)$ (respectively $\left.{ }_{\mathbb{A}} N^{\prime}\left(\lambda, \lambda^{\prime}\right)\right)$ of $N\left(\lambda, \lambda^{\prime}\right)$ (respectively $\left.N^{\prime}\left(\lambda, \lambda^{\prime}\right)\right)$.

Let ${ }^{-}: V\left(\lambda^{\prime}\right) \rightarrow V\left(\lambda^{\prime}\right)$ be the unique $\mathbb{Q}^{\pi}$-linear involution such that $\overline{u \eta_{\lambda^{\prime}}}=\bar{u} \eta_{\lambda^{\prime}}$ for all $u \in \mathbf{U}$; similarly, let $-:{ }^{\omega} V(\lambda) \rightarrow{ }^{\omega} V(\lambda)$ be the unique $\mathbb{Q}^{\pi}$-linear involution such that $\overline{u \xi_{-\lambda}}=\bar{u} \xi_{-\lambda}$ for all $u \in \mathbf{U}$. Let ${ }^{-}={ }^{-} \otimes^{-}: N\left(\lambda, \lambda^{\prime}\right) \rightarrow N\left(\lambda, \lambda^{\prime}\right)$. 
Since the maps $\Theta,^{-}: N\left(\lambda, \lambda^{\prime}\right) \rightarrow N\left(\lambda, \lambda^{\prime}\right)$ are well-defined, let $\Psi=\Theta_{\circ}{ }^{-}$. Then note that

$$
\Psi\left(\Delta(u) m \otimes m^{\prime}\right)=\Theta\left(\overline{\Delta(u) m \otimes m^{\prime}}\right)=\Delta(\bar{u}) \Theta\left(\bar{m} \otimes \overline{m^{\prime}}\right)=\Delta(\bar{u}) \Psi\left(m \otimes m^{\prime}\right),
$$

and that $\Psi^{2}=1$, from whence we call $\Psi$ the bar-involution on $N\left(\lambda, \lambda^{\prime}\right)$.

\subsection{The canonical basis of $N\left(\lambda, \lambda^{\prime}\right)$}

Now we have the necessary machinery to define a canonical basis on each module $N\left(\lambda, \lambda^{\prime}\right)$ using the bar-involution $\Psi$ and the $\pi$-basis $\mathbb{B}(\lambda) \otimes \mathbb{B}\left(\lambda^{\prime}\right)$. First, we present an analogue of $[\mathrm{Lu} 4, \S 24.2]$ in the covering setting.

Lemma 5.12. Let $H$ be a set with a partial order $\leq$ such that for any $h \leq h^{\prime}$ in $H$, the set $\left\{h^{\prime \prime}: h \leq h^{\prime \prime} \leq h^{\prime}\right\}$ is finite. Assume that for each $h \leq h^{\prime}$ in $H$, there exists an element $r_{h, h^{\prime}} \in \mathbb{A}$ such that $r_{h, h}=1$ and

$$
\sum_{h^{\prime \prime} ; h \leq h^{\prime \prime} \leq h^{\prime}} \bar{r}_{h, h^{\prime \prime}} r_{h^{\prime \prime}, h^{\prime}}=\delta_{h, h^{\prime}}
$$

for all $h \leq h^{\prime} \in H$.

Then there is a unique family of elements $p_{h, h^{\prime}} \in \mathbb{Z}[q]^{\pi}$ defined for all $h \leq h^{\prime} \in H$ such that $p_{h, h}=1, p_{h, h^{\prime}} \in q \mathbb{Z}[q]^{\pi}$ for all $h<h^{\prime}$ in $H$, and

$$
p_{h, h^{\prime}}=\sum_{h^{\prime \prime} ; h \leq h^{\prime \prime} \leq h^{\prime}} \bar{p}_{h, h^{\prime \prime}} r_{h^{\prime \prime}, h^{\prime}}
$$

for all $h \leq h^{\prime} \in H$.

Proof. For $h \leq h^{\prime}$ in $H$, denote by $d\left(h, h^{\prime}\right)$ the maximum length of a chain $h=h_{0}<$ $h_{1}<\ldots<h_{\ell}=h^{\prime} \in H$. Note that $d\left(h, h^{\prime}\right)<\infty$ by our assumption on $H$. For any $n \geq 0$, let $P_{n}$ be the assertion of the lemma restricted to those $h \leq h^{\prime}$ such that $d\left(h, h^{\prime}\right) \leq n$ (and note that all the assertions make sense under this restriction. We will prove $P_{n}$ by induction. 
First note $P_{0}$ is trivial and assume that $n \geq 1$. Let $h \leq h^{\prime}$. If $d\left(h, h^{\prime}\right)<n$ then $p_{h, h^{\prime}}$ is defined by $P_{n-1}$. If $d\left(h, h^{\prime}\right)=n$, then $z=\sum_{h^{\prime \prime} ; h \leq h^{\prime \prime}<h^{\prime}} \bar{p}_{h, h^{\prime \prime}} r_{h^{\prime \prime}, h^{\prime}}$ is defined.

First, we shall show that $z+\bar{z}=0$. Indeed, using $P_{n-1}$ and the assumptions of the lemma,

$$
\begin{aligned}
z+\bar{z} & =\sum_{h_{1} ; h \leq h_{0}<h^{\prime}} \bar{p}_{h, h_{0}} r_{h_{0}, h^{\prime}}+\sum_{h_{1} ; h \leq h_{1}<h^{\prime}} p_{h, h_{1}} \bar{r}_{h_{1}, h^{\prime}} \\
& =\sum_{h_{0}, h_{1} ; h \leq h_{0}<h_{1}=h^{\prime}} \bar{p}_{h, h_{0}} r_{h_{0}, h_{1}} \bar{r}_{h_{1}, h^{\prime}}+\sum_{h_{0}, h_{1} ; h \leq h_{0} \leq h_{1}<h^{\prime}} \bar{p}_{h, h_{0}} r_{h_{0}, h_{1}} \bar{r}_{h_{1}, h^{\prime}} \\
& =\sum_{h_{0}, h_{1} ; h \leq h_{0} \leq h_{1} \leq h^{\prime} ; h_{0}<h^{\prime}} \bar{p}_{h, h_{0}} r_{h_{0}, h_{1}} \bar{r}_{h_{1}, h^{\prime}} \\
& =\sum_{h_{0} ; h \leq h_{0}<h^{\prime}} \bar{p}_{h, h_{0}} \sum_{h_{1} ; h_{0} \leq h_{1} \leq h^{\prime}} \bar{r}_{h_{0}, h_{1}} r_{h_{1}, h^{\prime}} \\
& =\sum_{h_{0} ; h \leq h_{0}<h^{\prime}} \bar{p}_{h, h_{0}} \delta_{h_{0}, h^{\prime}}=0 .
\end{aligned}
$$

Now we claim that since $\bar{z}+z=0$, there is a unique $z^{\prime} \in q \mathbb{Z}[q]^{\pi}$ such that $z^{\prime}-\overline{z^{\prime}}=0$.

Indeed, we can write $z=\sum_{m, n \in \mathbb{N}} a_{n} q^{n}$ with $a_{n} \in \mathbb{Z}^{\pi}$ for $n \in \mathbb{N}$. Then since $z+\bar{z}=0$, we see that $a_{n}=-\pi^{n} a_{-n}$ for all $m, n \in \mathbb{N}$. In particular, $a_{0}=0$ and $a_{n}=0$ if and only if $a_{-n}=0$ for $n \in \mathbb{Z}$. Then taking $z^{\prime}=\sum_{n \in \mathbb{N}} a_{n} q^{n}$, we see that $z=z^{\prime}-\overline{z^{\prime}}$ and $z^{\prime} \in q \mathbb{Z}[q]^{\pi}$. In particular, we can set $p_{h, h^{\prime}}=z^{\prime}$, and then

$$
p_{h, h^{\prime}}=z+\overline{z^{\prime}}=\sum_{h^{\prime \prime} ; h \leq h^{\prime \prime}<h^{\prime}} \bar{p}_{h, h^{\prime \prime}} r_{h^{\prime \prime}, h^{\prime}}+\overline{p_{h, h^{\prime}}}=\sum_{h^{\prime \prime} ; h \leq h^{\prime \prime} \leq h^{\prime}} \bar{p}_{h, h^{\prime \prime}} r_{h^{\prime \prime}, h^{\prime}}
$$

Remark 5.13. For $R$ a commutative ring, we can define a bar-involution on $A=$ $R[x, y]$ by $\overline{f(x, y)}=f(y, x)$. This involutions descends to a bar-involution on $A_{r}=$ $R[x, y] /(x y-r) R[x, y] \cong R\left[x, r x^{-1}\right]$ for any $r \in R$. Then the assertions of the lemma apply with $\mathbb{A}$ replaced by $A$ or $A_{r}$, q replaced with $x$, and with $\mathbb{Z}[q]^{\pi}$ replaced everywhere by $R[x]$. In particular, Lemma 5.12 (respectively, [Lu4, §24.2]) is a special case for $R=\mathbb{Z}^{\pi}$ and $r=\pi$ (respectively, $R=\mathbb{Z}$ and $r=1$ ). 
Let $\lambda, \lambda^{\prime} \in X^{+}$. We shall consider the following partial order on the set $\mathbb{B} \times_{\pi} \mathbb{B}$ : we say that $\left(b_{1}, b_{1}^{\prime}\right) \leq\left(b_{2}, b_{2}^{\prime}\right)$ if

$$
\mathrm{ht}\left|b_{1}\right|-\mathrm{ht}\left|b_{1}^{\prime}\right|=\mathrm{ht}\left|b_{2}\right|-\mathrm{ht}\left|b_{2}^{\prime}\right|
$$

and if we have either

$$
\mathrm{ht}\left|b_{1}\right|<\mathrm{ht}\left|b_{2}\right| \text { and ht }\left|b_{1}^{\prime}\right|<\mathrm{ht}\left|b_{2}^{\prime}\right|
$$

or

$$
\left(b_{1}, b_{2}\right) \sim\left(b_{1}^{\prime}, b_{2}^{\prime}\right)
$$

Here, $\sim$ is as in (3.28). Note that, in particular, $\left(b_{1}, b_{1}^{\prime}\right)$ and $\left(b_{1}, \pi b_{1}^{\prime}\right)$ are not comparable under $\leq$. For given $\lambda, \lambda^{\prime} \in X^{+}$, this induces a partial order on the set $\mathbb{B}(\lambda) \times_{\pi} \mathbb{B}\left(\lambda^{\prime}\right)$.

Then from the definition, we have that for all $\left(b_{1}, b_{1}^{\prime}\right) \in B(\lambda) \times_{\pi} B\left(\lambda^{\prime}\right)$,

$$
\Psi\left(b_{1}^{-} \eta_{\lambda} \otimes b_{1}^{\prime+} \xi_{-\lambda^{\prime}}\right)=\sum_{\left(b_{2}, b_{2}^{\prime}\right) \in B(\lambda) \times_{\pi} B\left(\lambda^{\prime}\right)} r_{b_{1}, b_{1}^{\prime} ; b_{2}, b_{2}^{\prime}} b_{2}^{-} \eta_{\lambda} \otimes b_{2}^{\prime+} \xi_{-\lambda^{\prime}}
$$

where $r_{b_{1}, b_{1}^{\prime} ; b_{2}, b_{2}^{\prime}} \in \mathbb{Z}\left[q, q^{-1}\right]$ and $r_{b_{1}, b_{1}^{\prime} ; b_{2}, b_{2}^{\prime}}=0$ unless $\left(b_{1}, b_{1}^{\prime}\right) \geq\left(b_{2}, b_{2}^{\prime}\right)$; in particular, the sum is finite.

Moreover, we note that $r_{b_{1}, b_{1}^{\prime} ; b_{1}, b_{1}^{\prime}}=1$, and from $\Psi^{2}=1$ we see that

$$
\sum_{\left(b_{1}, b_{1}^{\prime}\right) ;\left(b_{2}, b_{2}^{\prime}\right) ;\left(b_{3}, b_{3}^{\prime}\right) \in B(\lambda) \times_{\pi} B\left(\lambda^{\prime}\right)} \bar{r}_{b_{1}, b_{1}^{\prime} ; b_{2}, b_{2}^{\prime}} r_{b_{2}, b_{2}^{\prime} ; b_{3}, b_{3}^{\prime}}=\delta_{\left(b_{1}, b_{1}^{\prime}\right) ;\left(b_{3}, b_{3}^{\prime}\right)} .
$$

Then $H=B(\lambda) \times_{\pi} B\left(\lambda^{\prime}\right)$ and $r_{b_{1}, b_{1}^{\prime} ; b_{2}, b_{2}^{\prime}}$ satisfy the assumptions of Lemma 5.12, so there exist elements $p_{b_{1}, b_{1}^{\prime} ; b_{2}, b_{2}^{\prime}} \in \mathbb{Z}[q]^{\pi}$ such that

$$
\begin{aligned}
& p_{b_{1}, b_{1}^{\prime} ; b_{1}, b_{1}^{\prime}}=1 \\
& p_{b_{1}, b_{1}^{\prime} ; b_{2}, b_{2}^{\prime}}=0 \text { unless }\left(b_{2}, b_{2}^{\prime}\right) \leq\left(b_{1}, b_{1}^{\prime}\right), \\
& p_{b_{1}, b_{1}^{\prime} ; b_{2}, b_{2}^{\prime}} \in q \mathbb{Z}[q]^{\pi} \text { for }\left(b_{2}, b_{2}^{\prime}\right)<\left(b_{1}, b_{1}^{\prime}\right), \\
& p_{b_{1}, b_{1}^{\prime} ; b_{3}, b_{3}^{\prime}}=\sum_{\left(b_{2}, b_{2}^{\prime}\right) \in B(\lambda) \times_{\pi} B\left(\lambda^{\prime}\right)} \bar{p}_{b_{1}, b_{1}^{\prime} ; b_{2}, b_{2}^{\prime}} r_{b_{2}, b_{2}^{\prime} ; b_{3}, b_{3}^{\prime}} .
\end{aligned}
$$


Recall from (5.6) that $\mathbb{B}(\lambda) \otimes \mathbb{B}\left(\lambda^{\prime}\right)=\left\{b^{+} \xi_{-\lambda} \otimes b^{-} \eta_{\lambda^{\prime}}: b \times b^{\prime} \in B(\lambda) \times_{\pi} B\left(\lambda^{\prime}\right)\right\}$, and we that $\mathcal{L}\left(\right.$ resp. ${ }_{\mathbb{A}} \mathcal{L}$ ) denotes the $\mathbb{Z}[q]^{\pi}$-lattice (resp. $\mathbb{A}$-lattice) spanned by this basis.

Theorem 5.14. 1. For any $\left(b, b^{\prime}\right) \in B(\lambda) \times_{\pi} B\left(\lambda^{\prime}\right)$, there is a unique element $\left(b \diamond b^{\prime}\right)_{\lambda, \lambda^{\prime}}$ of the $\mathbf{U}$-module $N\left(\lambda, \lambda^{\prime}\right)$ such that

$$
\Psi\left(\left(b \diamond b^{\prime}\right)_{\lambda, \lambda^{\prime}}\right)=\left(b \diamond b^{\prime}\right)_{\lambda, \lambda^{\prime}} \text { and }\left(b \diamond b^{\prime}\right)_{\lambda, \lambda^{\prime}}-b^{-} \eta_{-\lambda} \otimes b^{\prime+} \xi_{-\lambda^{\prime}} \in q \mathcal{L} .
$$

2. We have $\left(\pi b \diamond b^{\prime}\right)_{\lambda, \lambda^{\prime}}=\left(b \diamond \pi b^{\prime}\right)_{\lambda, \lambda^{\prime}}=\pi\left(b \diamond b^{\prime}\right)_{\lambda, \lambda^{\prime}}$

3. $\left(b \diamond b^{\prime}\right)_{\lambda, \lambda^{\prime}}$ is equal to $b^{-} \eta_{\lambda} \otimes b^{\prime+} \xi_{-\lambda^{\prime}}$ plus a $q \mathbb{Z}[q]^{\pi}$-linear combination of elements $b_{1}^{-} \eta_{\lambda} \otimes b_{1}^{\prime+} \xi_{-\lambda^{\prime}}$ with $\left(b_{1}, b_{1}^{\prime}\right)<\left(b, b^{\prime}\right)$.

4. The elements $\left(b \diamond b^{\prime}\right)_{\lambda, \lambda^{\prime}}$ form a $\pi$-basis of $\mathcal{L},{ }_{\mathbb{A}} \mathcal{L}$, and $N\left(\lambda, \lambda^{\prime}\right)$.

5. The natural homomorphism $\mathcal{L} \cap \Psi(\mathcal{L}) \rightarrow \mathcal{L} / q \mathcal{L}$ is an isomorphism.

Proof. By the definition of $p_{b, b^{\prime} ; b_{1}, b_{1}^{\prime}}$, we see that

$$
\left(b \diamond b^{\prime}\right)_{\lambda, \lambda^{\prime}}=\sum_{\left(b_{1}, b_{1}^{\prime}\right) \leq\left(b, b^{\prime}\right)} p_{b, b^{\prime} ; b_{1}, b_{1}^{\prime}} b_{1}^{-} \eta_{\lambda} \otimes b_{1}^{\prime+} \xi_{-\lambda^{\prime}}
$$

satisfies the requirements of (1) proving existence, and the same considerations prove (3). (4) is immediate from the fact that the transition matrix from the $\mathbb{Q}(q)$-basis $b_{1}^{-} \eta_{\lambda} \otimes b_{1}^{\prime+} \xi_{-\lambda^{\prime}}$ is unitriangular with entries in $\mathbb{Z}[q]$. (5) follows from (4) and the observation that $\left(b \diamond b^{\prime}\right)_{\lambda, \lambda^{\prime}} \in \mathcal{L} \cap \Psi(\mathcal{L})$, and so the map sends $\left(b \diamond b^{\prime}\right)_{\lambda, \lambda^{\prime}}$ to the basis element $b^{-} \eta_{\lambda} \otimes b^{\prime+} \xi_{-\lambda^{\prime}}$ of $\mathcal{L} / q \mathcal{L}$, which also implies uniqueness. Finally, uniqueness implies (2).

We call the elements $\left(b \diamond b^{\prime}\right)_{\lambda, \lambda^{\prime}}$ the canonical basis of $N\left(\lambda, \lambda^{\prime}\right)$.

Remark 5.15. We may repeat verbatim $§ 5.2$ with respect to $\Theta^{\prime}$ defined in Corollary 3.35 to obtain a bar-involution $\Psi^{\prime}$ on $N^{\prime}\left(\lambda, \lambda^{\prime}\right)$. Then we see that the results of this section (and in particular Theorem 5.14) may be restated with $\Psi, N\left(\lambda, \lambda^{\prime}\right)$ replaced by $\Psi^{\prime}, N^{\prime}\left(\lambda, \lambda^{\prime}\right)$. When we need to distinguish them, we denote the canonical basis of $N^{\prime}\left(\lambda, \lambda^{\prime}\right)$ by $\left(b \diamond^{\prime} b^{\prime}\right)_{\lambda, \lambda^{\prime}}$. 


\subsection{Cancellation and stability}

Our goal in this subsection is to exhibit maps between modules of the form $N\left(\lambda, \lambda^{\prime}\right)$ which are compatible with their canonical bases. These maps will correspond to a form of cancellation on the pairs $\left(\lambda, \lambda^{\prime}\right) \in X^{+} \times X^{+}$; namely, the cancellation

$$
\left(\lambda+\lambda^{\prime \prime}, \lambda^{\prime \prime}+\lambda^{\prime}\right) \mapsto\left(\lambda, \lambda^{\prime}\right)
$$

can be realized as a $\mathbf{U}$-module homomorphism $N\left(\lambda+\lambda^{\prime \prime}, \lambda^{\prime \prime}+\lambda^{\prime}\right) \rightarrow N\left(\lambda, \lambda^{\prime}\right)$.

To construct this, first we must construct maps to pull apart the weights, in some sense. We already have these in the form of the maps $\chi\left(\lambda, \lambda^{\prime}\right)$ defined in $\S 4.4$.

Proposition 5.16. Let $\lambda, \lambda^{\prime} \in X^{+}$. Write $\eta=\eta_{\lambda}, \eta^{\prime}=\eta_{\lambda^{\prime}}$, and $\eta^{\prime \prime}=\eta_{\lambda+\lambda^{\prime}}$.

1. There is a unique homomorphism of $\mathbf{U}$-modules $\chi: V\left(\lambda+\lambda^{\prime}\right) \rightarrow V(\lambda) \otimes V\left(\lambda^{\prime}\right)$ such that $\chi\left(\eta^{\prime \prime}\right)=\eta \otimes \eta^{\prime}$.

2. Let $b \in B\left(\lambda+\lambda^{\prime}\right)$. We have $\chi\left(b^{-} \eta^{\prime \prime}\right)=\sum_{b_{1}, b_{2}} f\left(b ; b_{1}, b_{2}\right) b_{1}^{-} \eta \otimes b_{2}^{-} \eta^{\prime}$ where the sum is over $\left(b_{1}, b_{2}\right) \in B(\lambda) \times_{\pi} B\left(\lambda^{\prime}\right)$ and $f\left(b ; b_{1}, b_{2}\right) \in \mathbb{Z}[q]$.

3. If $b^{-} \eta^{\prime} \neq 0$, then $f(b ; b, 1)=1$ and $f\left(b ; b_{1}, 1\right)=0$ for any $b_{1} \neq b$. If $b^{-} \eta^{\prime}=0$, then $\left.f\left(b ; b_{1}, 1\right)\right)=0$ for any $b_{1}$.

Proof. We already defined such a map in $\S 4.4$; that is, $\chi=\chi\left(\lambda, \lambda^{\prime}\right)$. Since $\chi\left(\lambda, \lambda^{\prime}\right)$ preserves the crystal lattice and $\mathbb{B} \subset_{\mathbb{A}} \mathbf{f}$, we see that $f\left(b ; b_{1}, b_{2}\right) \in \mathbb{Z}[q]^{\pi} ;$ moreover, up to identifying $\pi\left(b_{1}^{-} \eta \otimes b_{2}^{-} \eta^{\prime}\right)$ with $\left(\pi b_{1}^{-}\right) \eta \otimes b_{2}^{-} \eta^{\prime}$, we may assume that $f\left(b ; b_{1}, b_{2}\right) \in \mathbb{Z}[q]$. (3) is immediate from the definition of the coproduct.

Proposition 5.17. Let $\lambda, \lambda^{\prime} \in X^{+}$. Write $\xi=\xi_{-\lambda}, \xi^{\prime}=\xi_{-\lambda^{\prime}}$, and $\xi^{\prime \prime}=\xi_{-\lambda-\lambda^{\prime}}$.

1. There is a unique homomorphism of $\mathbf{U}$-modules ${ }^{\omega} \chi:{ }^{\omega} V\left(\lambda+\lambda^{\prime}\right) \rightarrow{ }^{\omega} V\left(\lambda^{\prime}\right) \otimes^{\prime}$ ${ }^{\omega} V(\lambda)$ such that ${ }^{\omega} \chi\left(\xi^{\prime \prime}\right)=\xi^{\prime} \otimes \xi$. 
2. Let $b \in \mathbb{B}\left(\lambda+\lambda^{\prime}\right)_{\nu}$. We have

$$
{ }^{\omega} \chi\left(b^{+} \xi^{\prime \prime}\right)=\sum_{b_{1}, b_{2}} \pi^{p\left(b_{1}\right) p\left(b_{2}\right)} f\left(b ; b_{1}, b_{2}\right) b_{2}^{+} \xi^{\prime} \otimes b_{1}^{+} \xi
$$

where the sum is over $\left(b_{1}, b_{2}\right) \in B(\lambda) \times_{\pi} B\left(\lambda^{\prime}\right)$ and $f\left(b ; b_{1}, b_{2}\right) \in \mathbb{Z}[q]^{\pi}$.

3. If $b^{+} \xi^{\prime} \neq 0$, then $f(b ; b, 1)=1$ and $f\left(b ; b_{1}, 1\right)=0$ for any $b_{1} \neq b$. If we have $b^{+} \xi^{\prime}=0$, then $\left.f\left(b ; b_{1}, 1\right)\right)=0$ for any $b_{1}$.

Proof. Proposition 5.16can be entirely rewritten in terms of the coproduct $\Delta^{\prime}$ and the map $\chi^{\prime}\left(\lambda, \lambda^{\prime}\right)$. Moreover $\chi^{\prime}\left(\lambda, \lambda^{\prime}\right)$ can be viewed as a homomorphism

$$
{ }^{\omega} V\left(\lambda+\lambda^{\prime}\right) \rightarrow{ }^{\omega}\left(V(\lambda) \otimes_{4} V\left(\lambda^{\prime}\right)\right) .
$$

By Lemma 3.19, we have an isomorphism

$$
{ }^{\omega}\left(V(\lambda) \otimes_{4} V\left(\lambda^{\prime}\right)\right) \rightarrow{ }^{\omega} V\left(\lambda^{\prime}\right) \otimes{ }^{\omega} V(\lambda) \quad y \otimes z \mapsto \pi^{p(y) p(z)} z \otimes y
$$

Then taking ${ }^{\omega} \chi$ to be the composition of these homomorphisms proves (1). The remaining properties follow by the definitions.

Now we define a pairing on $N(\lambda, \lambda)$ which will allow us to "cancel" $V(\lambda)$ and ${ }^{\omega} V(\lambda)$.

Proposition 5.18. Let $\eta=\eta_{\lambda}$ and $\xi=\xi_{-\lambda}$.

1. There is a unique homomorphism of $\mathbf{U}$-modules $\delta_{\lambda}: N(\lambda, \lambda) \rightarrow \mathbb{Q}(q)^{\pi}$, where $\mathbb{Q}(q)^{\pi}$ is a $\mathbf{U}$-module under the counit map $\varsigma$, such that $\delta_{\lambda}(\xi \otimes \eta)=1$.

2. Let $b, b^{\prime} \in B(\lambda)$. Then $\delta_{\lambda}\left(b^{+} \xi \otimes b^{-} \eta\right)=1$ if $b=b^{\prime}=1$ and is in $q \mathbb{Z}[q]^{\pi}$ otherwise.

Proof. For such a map to exist, we would need

$$
\Delta\left(E_{i}\right)(x \otimes y)=E_{i} x \otimes \tilde{K}_{i} y+\pi_{i}^{p(x)} x \otimes F_{i} y \in \operatorname{ker}\left(\delta_{\lambda}\right) \text { for all } x \otimes y \in N(\lambda, \lambda)
$$


$\Delta\left(F_{i}\right)(x \otimes y)=F_{i} x \otimes y+\pi_{i}^{p(x)} \tilde{J}_{i} \tilde{K}_{i} x \otimes \pi_{i} \tilde{J}_{i} E_{i} y \in \operatorname{ker}\left(\delta_{\lambda}\right)$ for all $x \otimes y \in N(\lambda, \lambda)$

$$
\begin{gathered}
\Delta\left(K_{\mu}-1\right)(x \otimes y)=K_{\mu} x \otimes K_{-\mu} y-x \otimes y \in \operatorname{ker}\left(\delta_{\lambda}\right) \text { for all } x \otimes y \in N(\lambda, \lambda) \\
\Delta\left(J_{\mu}-1\right)(x \otimes y)=J_{\mu} x \otimes J_{\mu} y-x \otimes y \in \operatorname{ker}\left(\delta_{\lambda}\right) \text { for all } x \otimes y \in N(\lambda, \lambda)
\end{gathered}
$$

The following statement is equivalent to (1). There is a unique bilinear pairing $[-,-]$ : $V(\lambda) \times V(\lambda) \rightarrow \mathbb{Q}(q)^{\pi}$ satisfying $[\eta, \eta]=1$

$$
\begin{gathered}
{\left[E_{i} x, \tilde{K}_{i} y\right]=-\pi_{i}^{p(x)}\left[x, F_{i} y\right], \quad\left[F_{i} x, y\right]=-\pi_{i}^{p(x)}\left[\tilde{J}_{i} \tilde{K}_{i} x, \pi_{i} \tilde{J}_{i} E_{i} y\right]} \\
{\left[K_{\mu} x, y\right]=\left[x, K_{\mu} y\right], \quad\left[J_{\mu} x, y\right]=\left[x, J_{\mu} y\right] .}
\end{gathered}
$$

We may rewrite the conditions as

$$
\begin{gathered}
{\left[E_{i} x, y\right]=-\pi_{i}^{p(x)}\left[x, F_{i} \tilde{K}_{-i} y\right], \quad\left[F_{i} x, y\right]=-\pi_{i}^{p(x)}\left[x, \pi_{i} \tilde{K}_{i} F_{i} y\right]} \\
{\left[K_{\mu} x, y\right]=\left[x, K_{\mu} y\right], \quad\left[J_{\mu} x, y\right]=\left[x, J_{\mu} y\right] .}
\end{gathered}
$$

Let $\mathbf{s}: \mathbf{U} \rightarrow \mathbf{U}$ be the map defined by

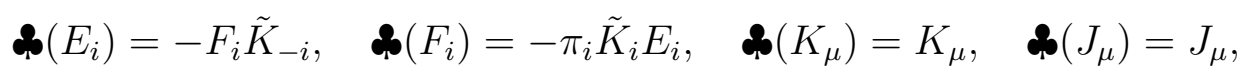

$$
\begin{aligned}
& \boldsymbol{\beta}(x y)=\pi^{p(x) p(y)} \boldsymbol{\beta}(y) \mathbf{\beta}(x) \text {. }
\end{aligned}
$$

To see this is a well-defined map, we note that $\boldsymbol{\phi}=\omega S$ where $S$ is the antipode defined in $\S 3.2$.

Then we see that (1) is equivalent to proving the existence of a unique bilinear pair$\operatorname{ing}[-,-]: V(\lambda) \times V(\lambda) \rightarrow \mathbb{Q}(q)^{\pi}$ satisfying $[\eta, \eta]=1$ and $[u x, y]=\pi^{p(x) p(u)}[x, \boldsymbol{\beta}(u) y]$. This follows by standard argument (i.e. the restricted dual $V(\lambda)^{*}$ has a $\mathbf{U}$-action $(u f)(x)=\pi^{p(u) p(f)} f(\boldsymbol{\beta}(x) u)$ under which we have an isomorphism $V(\lambda)^{*} \cong V(\lambda)$, and $[-,-]$ is the natural pairing).

Let $(-,-)$ be the polarization on $V(\lambda)$. We show by induction on ht $\nu \geq 0$ that

$$
[x, y]=(-1)^{\mathrm{ht} \nu} \pi^{\mathbf{p}(\nu)} \pi_{\nu} q_{\nu}(x, y)
$$

for $x, y \in V(\lambda)_{\lambda-\nu}$, where here we set $\mathbf{p}\left(\sum_{t} i_{t}\right)=\sum_{s<t} p\left(i_{s}\right) p\left(i_{t}\right)$. 
This is obvious for $\nu=0$. Assume that ht $\nu \geq 1$. Then we can assume that $x=F_{i} x^{\prime}$ for some $i$ such that $\nu_{i}>0$. Then by induction, we compute

$$
\begin{aligned}
{[x, y] } & =\left[F_{i} x^{\prime}, y\right]=-\pi_{i}^{p\left(x^{\prime}\right)}\left[x^{\prime}, \pi_{i} \tilde{K}_{i} E_{i} y\right]=-\pi_{i}^{p(\nu-i)} \pi_{i}\left[x^{\prime}, \tilde{K}_{i} E_{i} y\right] \\
& =-\pi_{i}^{p(\nu-i)} \pi_{i}(-1)^{\mathrm{ht}(\nu-i)} \pi_{i}^{\mathbf{p}(\nu-i)} \pi_{\nu-i} q_{\nu-i}\left(x^{\prime}, \tilde{K}_{i} E_{i} y\right) \\
& =(-1)^{\mathrm{ht} \nu} \pi^{\mathbf{p}(\nu)} \pi_{\nu} q_{\nu}\left(F_{i} x^{\prime}, y\right)=(-1)^{\mathrm{ht} \nu} \pi^{\mathbf{p}(\nu)} \pi_{\nu} q_{\nu}(x, y) .
\end{aligned}
$$

This proves that (5.7) holds. Now recall from Lemma $4.50(2)$ that $\left(b \eta, b^{\prime} \eta\right) \in \mathbb{Z}[q]^{\pi}$ for any $b, b^{\prime} \in \mathbb{B}$. Combining this with (5.7), we see that (2) follows.

Let $\lambda, \lambda^{\prime}, \lambda^{\prime \prime} \in X^{+}$. We define a $\mathbf{U}$-module homomorphism

$$
t: N\left(\lambda+\lambda^{\prime}, \lambda^{\prime}+\lambda^{\prime \prime}\right) \rightarrow N\left(\lambda, \lambda^{\prime \prime}\right)
$$

defined as the composition

$$
t=\left(1 \otimes \delta_{\lambda} \otimes 1\right) \circ\left(\chi \otimes{ }^{\omega} \chi\right)
$$

Lemma 5.19. We have $\Psi t=t \Psi$.

Proof. We write $\eta=\eta_{\lambda+\lambda^{\prime}}$ and $\xi=\xi_{-\lambda^{\prime}-\lambda^{\prime \prime}}$. Since any element of $N\left(\lambda+\lambda^{\prime}, \lambda^{\prime} \otimes \lambda^{\prime \prime}\right)$ is of the form $u(\eta \otimes \xi)$, it is enough to check that

$$
t \Theta(\overline{u(\eta \otimes \xi)})=\Theta(\overline{t(u(\eta \otimes \xi))})
$$

Well, on one hand

$$
t \Theta(\overline{u(\eta \otimes \xi)})=t(u \Theta(\eta \otimes \xi))=u t((\eta \otimes \xi))=u\left(\eta_{\lambda} \otimes \xi_{-\lambda^{\prime \prime}}\right) .
$$

On the other hand,

$$
\Theta(\overline{t(u(\eta \otimes \xi))})=\Theta(\overline{u t(\eta \otimes \xi)})=u \Theta\left(\eta_{\lambda} \otimes \xi_{-\lambda^{\prime \prime}}\right)=u\left(\eta_{\lambda} \otimes \xi_{-\lambda^{\prime \prime}}\right)
$$

The lemma is proved. 
Lemma 5.20. 1. Let $b \in \mathbb{B}(\lambda)$ and $b^{\prime \prime} \in \mathbb{B}\left(\lambda^{\prime \prime}\right)$. Then

$$
t\left(b^{-} \eta_{\lambda+\lambda^{\prime}} \otimes b^{\prime \prime+} \xi_{-\lambda^{\prime}-\lambda^{\prime \prime}}\right)=b^{-} \eta_{\lambda} \otimes b^{\prime \prime+} \xi_{-\lambda^{\prime \prime}} \bmod q \mathcal{L}\left(\lambda, \lambda^{\prime \prime}\right)
$$

In particular, $t\left(\left(b \diamond b^{\prime}\right)_{\lambda+\lambda^{\prime}, \lambda^{\prime}+\lambda^{\prime \prime}}\right)=\left(b \diamond b^{\prime}\right)_{\lambda, \lambda^{\prime \prime}} \bmod q \mathcal{L}\left(\lambda, \lambda^{\prime \prime}\right)$.

2. Let $b \in \mathbb{B}\left(\lambda+\lambda^{\prime}\right)$ and $b^{\prime \prime} \in \mathbb{B}\left(\lambda^{\prime}+\lambda^{\prime \prime}\right)$. Assume that either $b \notin \mathbb{B}(\lambda)$ or $b^{\prime \prime} \notin \mathbb{B}\left(\lambda^{\prime \prime}\right)$. Then

$$
t\left(b^{-} \eta_{\lambda+\lambda^{\prime}} \otimes b^{\prime \prime+} \xi_{-\lambda^{\prime}-\lambda^{\prime \prime}}\right)=0 \bmod q \mathcal{L}\left(\lambda, \lambda^{\prime \prime}\right) .
$$

In particular, $t\left(\left(b \diamond b^{\prime}\right)_{\lambda+\lambda^{\prime}, \lambda^{\prime}+\lambda^{\prime \prime}}\right)=0 \bmod q \mathcal{L}\left(\lambda, \lambda^{\prime \prime}\right)$.

3. $t$ is surjective.

Proof. Parts (1) and (2) follow from Propositions 5.16-5.18 and the definition of $t$. In particular, note that $t\left(\eta_{\lambda+\lambda^{\prime}} \otimes \xi_{-\lambda^{\prime}-\lambda^{\prime \prime}}\right)=\eta_{\lambda} \otimes \xi_{-\lambda^{\prime \prime}}$, which generates $N\left(\lambda, \lambda^{\prime \prime}\right)$.

Proposition 5.21. 1. Let $b \in \mathbb{B}(\lambda)$ and $b^{\prime \prime} \in \mathbb{B}\left(\lambda^{\prime \prime}\right)$. Then

$$
t\left(\left(b \diamond b^{\prime}\right)_{\lambda+\lambda^{\prime}, \lambda^{\prime}+\lambda^{\prime \prime}}\right)=\left(b \diamond b^{\prime}\right)_{\lambda, \lambda^{\prime \prime}}
$$

2. Let $b \in \mathbb{B}\left(\lambda+\lambda^{\prime}\right)$ and $b^{\prime \prime} \in \mathbb{B}\left(\lambda^{\prime}+\lambda^{\prime \prime}\right)$. Assume that $b \notin \mathbb{B}(\lambda)$ or $b^{\prime \prime} \notin \mathbb{B}\left(\lambda^{\prime \prime}\right)$. Then

$$
t\left(\left(b \diamond b^{\prime}\right)_{\lambda+\lambda^{\prime}, \lambda^{\prime}+\lambda^{\prime \prime}}\right)=0 .
$$

Proof. The differences of the two sides of the claimed equalities in (1) and (2) lie in $q \mathcal{L}\left(\lambda, \lambda^{\prime \prime}\right)$ and are fixed by $\Psi$, hence the difference is zero.

\subsection{The canonical basis of $\dot{U}$}

We are now in a position to produce a canonical basis for $\mathbf{U}$ which descends to the canonical bases of $N\left(\lambda, \lambda^{\prime}\right)$.

Theorem 5.22. Let $\zeta \in X$ and $\left(b, b^{\prime \prime}\right) \in \mathbb{B} \times_{\pi} \mathbb{B}$. 
1. There is a unique element $u=b \diamond_{\zeta} b^{\prime \prime} \in{ }_{\mathbb{A}} \dot{\mathbf{U}}$ such that

$$
\Delta(u)\left(\eta_{\lambda} \otimes \zeta_{\lambda^{\prime \prime}}\right)=\left(b \diamond b^{\prime \prime}\right)_{\lambda, \lambda^{\prime \prime}}
$$

for any $\lambda, \lambda^{\prime \prime} \in X^{+}$such that $b \in B(\lambda), b^{\prime \prime} \in B\left(\lambda^{\prime \prime}\right)$, and $\zeta=\lambda-\lambda^{\prime \prime}$.

2. If $\lambda, \lambda^{\prime \prime} \in X^{+}$are such that $\lambda-\lambda^{\prime \prime}=\zeta$ and either $b \notin \mathbb{B}(\lambda)$ or $b^{\prime \prime} \notin \mathbb{B}\left(\lambda^{\prime \prime}\right)$, then

$$
\Delta\left(b \diamond_{\zeta} b^{\prime \prime}\right)\left(\eta_{\lambda} \otimes \xi_{-\lambda^{\prime \prime}}\right)=0
$$

3. $\overline{b \succ_{\zeta} b^{\prime \prime}}=b \zeta_{\zeta} b^{\prime \prime}$.

4. The elements $b \diamond_{\zeta} b^{\prime \prime}$, for various $\zeta, b, b^{\prime \prime}$ as above, form a $\mathbb{Q}(q)^{\pi}$-basis of $\dot{\mathbf{U}}$ and a $\mathbb{A}$-basis of ${ }_{\mathbb{A}} \dot{\mathbf{U}}$.

Proof. The proof of this theorem proceeds exactly as in [Lu4, Theorem 25.2.1]. We state it here for completeness.

First recall that we assume the root datum is $Y$-regular. Then we can find $\lambda, \lambda^{\prime \prime} \in$ $X^{+}$such that $b \in B(\lambda), b^{\prime \prime} \in B\left(\lambda^{\prime \prime}\right)$, and $\lambda-\lambda^{\prime \prime}=\zeta$.

For any integers $N_{1}, N_{2}$, let $P\left(N_{1}, N_{2}\right)$ be the $\mathbb{A}$-submodule of ${ }_{\mathbb{A}} \dot{U}$ spanned by the elements $b_{1}^{-} b_{2}^{+} 1_{\zeta}$ where $b_{1}$ and $b_{2}$ run through the elements of $\mathbb{B}$ such that ht $\left|b_{1}\right| \leq N_{1}$, ht $\left|b_{2}\right| \leq N_{2}$, and $\left|b_{1}\right|-\left|b_{2}\right|=|b|-\left|b^{\prime \prime}\right|$.

Recall that any element of $N\left(\lambda, \lambda^{\prime \prime}\right)$ of the form $\beta^{-} \eta_{\lambda} \otimes \beta^{\prime+} \xi_{-\lambda}$ with $\beta, \beta^{\prime} \in \mathbb{B}$ is equal to $u_{1}\left(\eta_{\lambda} \otimes \xi_{-\lambda}\right)$ for some $u_{1} \in P\left(\mathrm{ht}|\beta|\right.$, ht $\left.\left|\beta^{\prime}\right|\right)$; moreover, $u_{1}$ can be taken to be equal to $\beta^{-} \beta^{\prime+} 1_{\zeta}$ plus an element in $P\left(\mathrm{ht}|\beta|-1\right.$, ht $\left.\left|\beta^{\prime}\right|-1\right)$. In particular, we see that $\left(b \diamond b^{\prime \prime}\right)_{\lambda, \lambda^{\prime \prime}}$ is of the form $u\left(\eta_{\lambda} \otimes \xi_{-\lambda^{\prime \prime}}\right)$ for some $u \in P\left(\right.$ ht $|b|$, ht $\left.\left|b^{\prime \prime}\right|\right)$; moreover, $u$ can be taken to be equal to $b^{-} b^{\prime \prime+} 1_{\zeta}$ plus an element in $P\left(\right.$ ht $|b|-1$, ht $\left.\left|b^{\prime \prime}\right|-1\right)$.

Assume that $u$ is such an element and $u^{\prime}$ is another such element. Then $(u-$ $\left.u^{\prime}\right)\left(\eta_{\lambda} \otimes \xi_{-\lambda^{\prime \prime}}\right)=0$, and so by Proposition 5.8

$$
\left(u-u^{\prime}\right) \in \sum_{i, n>\langle i, \lambda\rangle}{ }_{\mathbb{A}} \dot{\mathbf{U}} F_{i}^{(n)} 1_{\zeta}+\sum_{i, n>\left\langle i, \lambda^{\prime \prime}\right\rangle}{ }_{\mathbb{A}} \dot{\mathbf{U}} E_{i}^{(n)} 1_{\zeta} .
$$


However, since $u-u^{\prime} \in P\left(\mathrm{ht}|b|, \mathrm{ht}\left|b^{\prime \prime}\right|\right)$, we must have $u-u^{\prime}=0$ if $\langle i, \lambda\rangle>\mathrm{ht}|b|$ and $\left\langle i, \lambda^{\prime \prime}\right\rangle>\mathrm{ht}\left|b^{\prime \prime}\right|$ for all $i \in I$. For such $\lambda, \lambda^{\prime \prime}$ the element $u$ is uniquely determined, and we denote it by $u_{\lambda, \lambda^{\prime \prime}}$.

Assume now that $\lambda, \lambda^{\prime \prime} \in X^{+}$satisfy $b \in \mathbb{B}(\lambda), b^{\prime \prime} \in \mathbb{B}\left(\lambda^{\prime \prime}\right)$, and $\lambda-\lambda^{\prime \prime}=\zeta$. Let $\lambda^{\prime} \in X^{+}$such that $\lambda>>0$ so that $u^{\prime}=u_{\lambda+\lambda^{\prime}, \lambda^{\prime}+\lambda^{\prime \prime}}$ is defined. Then

$$
u^{\prime}\left(\eta_{\lambda} \otimes \xi_{-\lambda^{\prime \prime}}\right)=u^{\prime} t\left(\eta_{\lambda+\lambda^{\prime}} \otimes \xi_{-\lambda^{\prime}-\lambda^{\prime \prime}}\right)=t\left(\left(b \diamond b^{\prime \prime}\right)_{\lambda+\lambda^{\prime}, \lambda^{\prime}+\lambda^{\prime \prime}}\right)=\left(b \diamond b^{\prime \prime}\right)_{\lambda, \lambda^{\prime \prime}}
$$

Then $u_{\lambda, \lambda^{\prime \prime}}$ is independent of $\lambda, \lambda^{\prime \prime}$ if it is defined, so we may denote it as $u$ without specifying $\lambda, \lambda^{\prime \prime}$. In particular, this element satisfies the requirements of (1), proving existence and uniqueness.

This argument also proves (2), since in this case we may pick $\lambda^{\prime}$ so that $b \in \mathbb{B}\left(\lambda+\lambda^{\prime}\right)$ and $b \in \mathbb{B}\left(\lambda^{\prime}+\lambda^{\prime \prime}\right)$, and then $t\left(\left(b \diamond b^{\prime \prime}\right)_{\lambda+\lambda^{\prime}, \lambda^{\prime}+\lambda^{\prime \prime}}\right)=0$.

The bar-invariance of the canonical basis of $N\left(\lambda, \lambda^{\prime \prime}\right)$ and the uniqueness of the element $u$ shows that $\bar{u}=u$, and hence (3) holds.

Finally, the uniqueness of $u$ forces $b \diamond_{\zeta} b^{\prime \prime}=b^{-} b^{\prime \prime+} 1_{\zeta}$ modulo $P\left(\right.$ ht $|b|-1$, ht $\left.\left|b^{\prime \prime}\right|-1\right)$ Since $b^{-} b^{\prime \prime+} 1_{\zeta}$ forms a basis of $\mathbf{U}$, the transition matrix from $b^{-} b^{\prime \prime+} 1_{\zeta}$ to $b \diamond_{\zeta} b^{\prime \prime}$ may be made upper unitriangular (by a suitable ordering), hence $b \diamond_{\zeta} b^{\prime \prime}$ forms a basis.

We let $\dot{\mathbb{B}}=\left\{\left(b \diamond_{\zeta} b^{\prime}:\left(b, b^{\prime}\right) \in \mathbb{B} \times_{\pi} \mathbb{B}, \zeta \in X\right\}\right.$ and call this the canonical basis of U.

Example 5.23. Suppose that $I=I_{\overline{1}}=\{i\}$. Then $\mathbb{B}=\left\{\pi^{\epsilon} \theta_{i}^{(a)}: a \in \mathbb{N}, \epsilon \in\{0,1\}\right\}$. Let $a, b \in \mathbb{N}$ and suppose that $n \geq a+b$. Then using similar computations as in the proof of [ClW, Theorem 6.2],

$$
\begin{aligned}
\left(\theta_{i}^{(a)} \diamond_{n-2 a} \theta_{i}^{(b)}\right) & =F_{i}^{(a)} E_{i}^{(b)} 1_{n} \\
\left(\theta_{i}^{(a)} \diamond_{2 b-n} \theta_{i}^{(b)}\right) & =\pi^{a b} E_{i}^{(b)} F_{i}^{(a)} 1_{-n} .
\end{aligned}
$$

We note that the though this $\pi$-basis matches the one in loc. cit., the $\left(\mathbb{B} \times_{\pi} \mathbb{B}\right) \times X$ labeling differs because several conventions differ. 
Since the canonical basis of $\dot{\mathbf{U}}$ descends to the canonical basis of $N\left(\lambda, \lambda^{\prime}\right)$, a natural question to ask is what this basis descends to on $N^{\prime}\left(\lambda, \lambda^{\prime}\right)$.

Corollary 5.24. Let $\zeta \in X$ and $\left(b, b^{\prime}\right) \in \mathbb{B} \times_{\pi} \mathbb{B}$. For any $\lambda, \lambda^{\prime} \in X^{+}$such that $b \in B(\lambda), b^{\prime} \in B\left(\lambda^{\prime}\right)_{\nu}$, and $\zeta=\lambda-\lambda^{\prime}$,

$$
\Delta^{\prime}\left(b \diamond \zeta b^{\prime}\right)\left(\eta_{\lambda} \otimes \xi_{-\lambda^{\prime}}\right)=\pi^{\langle\tilde{\nu}, \lambda\rangle}\left(b \diamond^{\prime} b^{\prime}\right)_{\lambda, \lambda^{\prime}}
$$

Proof. Let $u=b \diamond_{\zeta} b^{\prime}$. Then by definition, $\Delta(u) \eta_{\lambda} \otimes \xi_{-\lambda^{\prime \prime}}=\left(b \diamond b^{++}\right)_{\lambda, \lambda^{\prime}}$, and $\left(b \diamond b^{\prime}\right)_{\lambda, \lambda^{\prime}}-$ $b^{+} \eta_{\lambda} \otimes b^{-} \xi_{-\lambda^{\prime}} \in q \mathcal{L}$. Therefore, applying the isomorphism in Lemma 3.24 (which obviously preserves $\mathcal{L})$,

$$
\Delta^{\prime}(u) \eta_{\lambda} \otimes \xi_{-\lambda^{\prime \prime}}-\pi^{\langle\tilde{\nu}, \lambda\rangle} b^{+} \eta_{\lambda} \otimes b^{-} \xi_{-\lambda^{\prime}} \in q \mathcal{L}
$$

On the other hand, $\Psi^{\prime}\left(\Delta^{\prime}(u) \eta_{\lambda} \otimes \xi_{-\lambda^{\prime \prime}}\right)=\Delta^{\prime}(u) \eta_{\lambda} \otimes \xi_{-\lambda^{\prime \prime}}$ by the bar-invariance of $u$. Then by uniqueness, we have $\Delta^{\prime}(u)=\pi^{\langle\tilde{\nu}, \lambda\rangle}\left(b \diamond^{\prime} b^{\prime}\right)_{\lambda, \lambda^{\prime}}$.

Finally, recall that the twistor $\mathfrak{X}$ has an incarnation defined on $\dot{\mathbf{U}}[\mathbf{t}]$. Given our success at relating the canonical basis of $\mathbf{f}$ via a twistor isomorphism, it is reasonable to hope such a connection can be made for the canonical basis of $\dot{\mathbf{U}}$ we have just defined. This is in fact the case. Recall the maps $\mathfrak{X}_{\lambda, \lambda^{\prime}}: N\left(\lambda, \lambda^{\prime}\right) \rightarrow N\left(\lambda, \lambda^{\prime}\right)$ from $\S 3.6$.

Theorem 5.25. Let $b, b^{\prime} \in \mathcal{B}$ and $\lambda, \lambda^{\prime} \in X^{+}$. Set $\zeta=\lambda-\lambda^{\prime}$.

1. We have $\mathfrak{X}_{\lambda, \lambda^{\prime}}\left(\left(b \diamond b^{\prime}\right)_{\lambda, \lambda^{\prime}}\right)=\mathbf{t}^{f\left(b, b^{\prime}, \zeta\right)}\left(b \diamond b^{\prime}\right)_{\lambda, \lambda^{\prime}}$ for some $f\left(b, b^{\prime}, \zeta\right) \in \mathbb{Z} / 4 \mathbb{Z}$.

2. We have $\mathfrak{X}\left(b \diamond_{\zeta} b^{\prime}\right)=\mathbf{t}^{f\left(b, b^{\prime}, \zeta\right)}\left(b \diamond_{\zeta} b^{\prime}\right)$.

Proof. For (1), first note that the claim $\mathfrak{X}_{\lambda, \lambda^{\prime}}\left(\left(b \diamond b^{\prime}\right)_{\lambda, \lambda^{\prime}}\right)=\mathbf{t}^{f\left(b, b^{\prime}, \lambda, \lambda^{\prime}\right)}\left(b \diamond b^{\prime}\right)_{\lambda, \lambda^{\prime}}$ for some integer $f\left(b, b^{\prime}, \lambda, \lambda^{\prime}\right)$ follows by combining the definition of the canonical basis of $N\left(\lambda, \lambda^{\prime}\right)$ with Proposition 3.32, Lemma 3.26 and Theorem thm:CB comparison.

Let $u=b \diamond_{\zeta} b^{\prime}$. Then $\mathfrak{X}\left(u\left(\eta_{\lambda} \otimes \xi_{-\lambda^{\prime}}\right)\right)=\mathbf{t}^{f\left(b, b^{\prime}, \lambda, \lambda^{\prime}\right)}\left(b \diamond b^{\prime}\right)_{\lambda, \lambda^{\prime}}$; on the other hand, $\mathfrak{X}\left(u\left(\eta_{\lambda} \otimes \xi_{-\lambda^{\prime}}\right)\right)=\Delta(\mathfrak{X}(u))\left(\eta_{\lambda} \otimes \xi_{-\lambda^{\prime}}\right)$. Therefore, we see that

$$
\Delta(\mathfrak{X}(u))\left(\eta_{\lambda} \otimes \xi_{-\lambda^{\prime}}\right)=\mathbf{t}^{f\left(b, b^{\prime}, \lambda, \lambda^{\prime}\right)}\left(b \diamond b^{\prime}\right)_{\lambda, \lambda^{\prime}}
$$


Let $\lambda^{\prime \prime} \in X^{+}$. Then

$$
\begin{aligned}
\Delta(\mathfrak{X}(u))\left(\eta_{\lambda} \otimes \xi_{-\lambda^{\prime}}\right) & =\Delta(\mathfrak{X}(u)) t\left(\eta_{\lambda+\lambda^{\prime \prime}} \otimes \xi_{-\lambda^{\prime \prime}-\lambda^{\prime}}\right) \\
& =t\left(\Delta(\mathfrak{X}(u)) \eta_{\lambda+\lambda^{\prime \prime}} \otimes \xi_{-\lambda^{\prime \prime}-\lambda^{\prime}}\right) \\
& =t\left(\mathbf{t}^{f\left(b, b^{\prime}, \lambda+\lambda^{\prime \prime}, \lambda^{\prime \prime}+\lambda^{\prime}\right)}\left(b \diamond b^{\prime}\right)_{\lambda+\lambda^{\prime \prime}, \lambda^{\prime \prime}+\lambda^{\prime}}\right) \\
& =\mathbf{t}^{f\left(b, b^{\prime}, \lambda+\lambda^{\prime \prime}, \lambda^{\prime \prime}+\lambda^{\prime}\right)}\left(b \diamond b^{\prime}\right)_{\lambda, \lambda^{\prime}}
\end{aligned}
$$

In particular, we see that $f\left(b, b^{\prime}, \lambda, \lambda^{\prime}\right)=f\left(b, b^{\prime}, \lambda+\lambda^{\prime \prime}, \lambda^{\prime \prime}+\lambda^{\prime}\right) \operatorname{modulo} 4$, so $\mathbf{t}^{f\left(b, b^{\prime}, \lambda, \lambda^{\prime}\right)}$ is determined by $b, b^{\prime}$, and $\zeta=\lambda-\lambda^{\prime}$, which finishes the proof of (1).

In particular, setting $f\left(b, b^{\prime}, \zeta\right)=f\left(b, b^{\prime}, \lambda, \lambda^{\prime}\right)$,

$$
\Delta(\mathfrak{X}(u))\left(\eta_{\lambda_{0}} \otimes \xi_{-\lambda_{0}^{\prime}}\right)=\mathbf{t}^{f\left(b, b^{\prime}, \zeta\right)}\left(b \diamond b^{\prime}\right)_{\lambda_{0}, \lambda_{0}^{\prime}}
$$

for all $\lambda_{0}, \lambda_{0}^{\prime} \in X^{+}$with $\zeta=\lambda_{0}-\lambda_{0}^{\prime}$, so (2) follows by uniqueness.

\subsection{The bilinear form on U}

Now we will show that a bilinear form with several desirable properties can be defined on $\dot{\mathbf{U}}$.

Theorem 5.26. There exists a unique $\mathbb{Q}(q)^{\pi}$-bilinear form

$$
\{-,-\}: \dot{\mathbf{U}} \times \dot{\mathbf{U}} \rightarrow \mathbb{Q}(q)^{\pi}
$$

such that the following hold.

1. $\left\{1_{\lambda_{1}} x 1_{\lambda_{2}}, 1_{\lambda_{1}^{\prime}} x^{\prime} 1_{\lambda_{2}^{\prime}}\right\}$ is zero unless $\lambda_{1}=\lambda_{1}^{\prime}, \lambda_{2}=\lambda_{2}^{\prime}$;

2. $\{u x, y\}=\left\{x, \tau_{1}(u) y\right\}$ for all $x, y \in \dot{\mathbf{U}}$ and $u \in \mathbf{U}$;

3. $\left\{x^{-} 1_{\lambda}, x^{-} 1_{\lambda}\right\}=\left\{x, x^{\prime}\right\}$ for all $x, x^{\prime} \in \mathbf{f}$ and all $\lambda$;

4. We have $\{x, y\}=\{y, x\}$. 
Proof. The proof is virtually the same as [Lu4, Theorem 26.1.2]. In brief, one uses the triangular decomposition of $\dot{\mathbf{U}}$ and the bilinear form on $\mathbf{f}$ to construct a linear functional on $\dot{\mathbf{U}} 1_{\zeta}$ for each $\zeta \in X$. Then this functional can be used to define a bilinear form on each space, and the direct sum of these forms has the desired properties.

To develop the properties of this bilinear form, we need to understand the relations between compositions of some of the (anti-)automorphisms of $\mathbf{U}$. To that end, recall the maps $\varrho$ and $\omega$ from $\S 3.1 \tau_{1}$ from $\S 4.4$ and let $\bar{\tau}_{1}$ to be the map $u \mapsto \overline{\tau_{1}(\bar{u})}$; that is, the map satisfying

$$
\bar{\tau}_{1}\left(E_{i}\right)=\pi_{i} q_{i}^{-1} F_{i} \tilde{J}_{i} \tilde{K}_{i}, \quad \tau_{1}\left(F_{i}\right)=\pi_{i} q_{i}^{-1} E_{i} \tilde{J}_{i} \tilde{K}_{-i}, \quad \tau_{1}\left(K_{\mu}\right)=K_{\mu}, \quad \tau_{1}\left(J_{\mu}\right)=J_{\mu} .
$$

Lemma 5.27. We have the identities

$$
\varrho \tau_{1}=\bar{\tau}_{1} \varrho, \quad \omega^{-1} \tau_{1}=\tau_{1} \omega
$$

Proof. It suffices to check these identities on the generators, and therefore, since all compositions considered fix $J_{\mu}$ and $K_{\mu}$, we only need to check on $E_{i}$ and $F_{i}$ for $i \in I$.

Well,for $\varrho \tau_{1}=\bar{\tau}_{1} \varrho$ :

$$
\begin{gathered}
\varrho\left(\tau_{1}\left(E_{i}\right)\right)=\varrho\left(q_{i}^{-1} \tilde{K}_{-i} F_{i}\right)=q_{i}^{-1} F_{i} \tilde{K}_{i}, \quad \bar{\tau}_{1}\left(\varrho\left(E_{i}\right)=\bar{\tau}_{1}\left(\pi_{i} \tilde{J}_{i} E_{i}\right)=q_{i}^{-1} F_{i} \tilde{K}_{i},\right. \\
\varrho\left(\tau_{1}\left(F_{i}\right)\right)=\varrho\left(q_{i}^{-1} \tilde{K}_{i} E_{i}\right)=\pi_{i} q_{i}^{-1} E_{i} \tilde{J}_{i} \tilde{K}_{-i}, \quad \bar{\tau}_{1}\left(\varrho\left(F_{i}\right)=\bar{\tau}_{1}\left(E_{i}\right)=q_{i}^{-1} E_{i} \tilde{J}_{i} \tilde{K}_{-i} .\right.
\end{gathered}
$$

Checking the left-hand equations to the right-hand equations verifies the identity.

Similarly, for $\omega^{-1} \tau_{1}=\tau_{1} \omega$ :

$$
\begin{gathered}
\omega^{-1}\left(\tau_{1}\left(E_{i}\right)\right)=\omega^{-1}\left(q_{i}^{-1} \tilde{K}_{-i} F_{i}\right)=q_{i}^{-1} \tilde{K}_{i} E_{i}, \quad \tau_{1}\left(\omega\left(E_{i}\right)\right)=\tau_{1}\left(F_{i}\right)=q_{i}^{-1} \tilde{K}_{i} E_{i}, \\
\omega^{-1}\left(\tau_{1}\left(F_{i}\right)\right)=\omega\left(q_{i}^{-1} \tilde{K}_{i} E_{i}\right)=\pi_{i} q_{i}^{-1} \tilde{J}_{i} \tilde{K}_{-i} F_{i}, \quad \tau_{1}\left(\omega\left(F_{i}\right)=\tau_{1}\left(\pi_{i} \tilde{J}_{i} E_{i}\right)=\pi_{i} q_{i}^{-1} \tilde{J}_{i} \tilde{K}_{-i} F_{i} .\right.
\end{gathered}
$$

Comparing the left-hand equations to the right-hand equations verifies the identity.

Proposition 5.28. We have $\{x u, y\}=\left\{x, y \bar{\tau}_{1}(u)\right\}$ for all $x, y \in \dot{\mathbf{U}}, u \in \mathbf{U}$. 
Proof. It suffices to prove this for the generators; it is clear for $K_{\mu}, J_{\mu}$. It remains to verify that

$$
\left\{x E_{i}, y\right\}=\left\{x, \pi_{i} q_{i}^{-1} y F_{i} \tilde{J}_{i} \tilde{K}_{i}\right\}, \quad\left\{x F_{i}, y\right\}=\left\{x, \pi_{i} q_{i}^{-1} y E_{i} \tilde{J}_{i} \tilde{K}_{-i}\right\}
$$

We may further assume that $x=u^{\prime} 1_{\zeta}$ where $u^{\prime} \in U$ and $\zeta \in X$. Then setting $y^{\prime}=\tau_{1}\left(u^{\prime}\right) y$, we see that the previous equalities follow from

$$
\left\{1_{\zeta} E_{i}, y^{\prime}\right\}=\left\{1_{\zeta}, \pi_{i} q_{i}^{-1} y^{\prime} F_{i} \tilde{J}_{i} \tilde{K}_{i}\right\}, \quad\left\{x F_{i}, y\right\}=\left\{x, \pi_{i} q_{i}^{-1} y E_{i} \tilde{J}_{i} \tilde{K}_{-i}\right\} .
$$

Once more, we can assume that $y^{\prime}=\tau_{1}\left(y_{1}^{-}\right) y_{2}^{-} 1_{\zeta}^{\prime}$ for homogeneous $y_{1}, y_{2} \in \mathbf{f}$, so it suffices to show that

$$
\begin{aligned}
& \left\{y_{1}^{-} E_{i} 1_{\zeta-i^{\prime}}, y_{2}^{-} 1_{\zeta}^{\prime}\right\}=\pi_{i}^{1+\left\langle i, \zeta^{\prime}+i^{\prime}\right\rangle} q_{i}^{-1+\left\langle i, \zeta^{\prime}+i^{\prime}\right\rangle}\left\{y_{1}^{-} 1_{\zeta}, y_{2}^{-} F_{i} 1_{\zeta^{\prime}+i^{\prime}}\right\} \\
& \left\{y_{1}^{-} F_{i} 1_{\zeta+i^{\prime}}, y_{2}^{-} 1_{\zeta}^{\prime}\right\}=\pi_{i}^{1+\left\langle i, \zeta^{\prime}-i^{\prime}\right\rangle} q_{i}^{-1-\left\langle i, \zeta^{\prime}-i^{\prime}\right\rangle}\left\{y_{1}^{-} 1_{\zeta}, y_{2}^{-} E_{i} 1_{\zeta^{\prime}-i^{\prime}}\right\} .
\end{aligned}
$$

By symmetry, (a) and (b) are equivalent, so we shall prove (a). Then we may assume $\zeta^{\prime}=\zeta-i^{\prime}$ and $\left|y_{1}\right|=\left|y_{2}\right|+i$.

Using Proposition 3.6 we have

$$
E_{i} y_{1}^{-} 1_{\zeta^{\prime}}-\pi_{i}^{p\left(y_{1}\right)} y_{1}^{-} E_{i} 1_{\zeta^{\prime}}=\frac{\pi_{i}^{p\left(y_{1}\right)-p(i)} \overline{r_{i}}\left(y_{1}\right)^{-} \tilde{J}_{i} \tilde{K}_{i}-\tilde{K}_{-i} \bar{i}\left(y_{1}\right)^{-}}{\pi_{i} q_{i}-q_{i}^{-1}} 1_{\zeta},
$$

from whence we see that

$$
y_{1}^{-} E_{i} 1_{\zeta^{\prime}}=\pi_{i}^{p\left(y_{1}\right)} E_{i} y_{1}^{-} 1_{\zeta^{\prime}}+\frac{\pi_{i} \overline{r_{i}}\left(y_{1}\right)^{-} \tilde{J}_{i} \tilde{K}_{i}-\pi_{i}^{p\left(y_{1}\right)} \tilde{K}_{-i} \bar{i} r\left(y_{1}\right)^{-}}{q_{i}^{-1}-\pi_{i} q_{i}} 1_{\zeta} .
$$

Now note that

$$
\begin{aligned}
\left\{\pi_{i}^{p\left(y_{1}\right)} E_{i} y^{-} 1_{\zeta^{\prime}}, y_{2}^{-} 1_{\zeta^{\prime}}\right\} & =\pi_{i}^{p\left(y_{1}\right)} q_{i}^{1+\left\langle i,\left|y_{2}\right|-\zeta^{\prime}\right\rangle}\left\{y_{1}^{-} 1_{\zeta^{\prime}}, F_{i} y_{2}^{-} 1_{\zeta^{\prime}}\right\} \\
& =\pi_{i}^{p\left(y_{1}\right)} q_{i}^{1+\left\langle i,\left|y_{2}\right|-\zeta^{\prime}\right\rangle}\left\{y_{1}, \theta_{i} y_{2}\right\}
\end{aligned}
$$


and that

$$
\begin{aligned}
& \left\{\frac{\pi_{i} \bar{r}_{i}\left(y_{1}\right)^{-} \tilde{J}_{i} \tilde{K}_{i}-\pi_{i}^{p\left(y_{1}\right)} \tilde{K}_{-i} \bar{i}\left(y_{1}\right)^{-}}{q_{i}^{-1}-\pi_{i} q_{i}} 1_{\zeta}, y_{2}^{-} 1_{\zeta^{\prime}}\right\} \\
& =\left\{\theta_{i}, \theta_{i}\right\}\left\{\pi_{i}^{1+\left\langle i, \zeta^{\prime}\right\rangle} q_{i}^{1+\left\langle i, \zeta^{\prime}\right\rangle} \bar{r}_{i}\left(y_{1}\right)^{-} 1_{\zeta^{\prime}}-\pi_{i}^{p\left(y_{1}\right)} q_{i}^{1+\left\langle i,\left|y_{1}\right|-i-\zeta^{\prime}\right\rangle} \bar{i}_{i}\left(y_{1}\right)^{-} 1_{\zeta^{\prime}}, y_{2}^{-} 1_{\zeta^{\prime}}\right\} \\
& =\pi_{i}^{1+\left\langle i, \zeta^{\prime}\right\rangle} q_{i}^{1+\left\langle i, \zeta^{\prime}\right\rangle}\left\{\theta_{i}, \theta_{i}\right\}\left\{\bar{r}_{i}\left(y_{1}\right), y_{2}\right\}-\pi_{i}^{p\left(y_{1}\right)} q_{i}^{1+\left\langle i,\left|y_{1}\right|-i-\zeta^{\prime}\right\rangle}\left\{\theta_{i}, \theta_{i}\right\}\left\{{ }_{i}^{r}\left(y_{1}\right), y_{2}\right\} \\
& =\pi_{i}^{1+\left\langle i, \zeta^{\prime}\right\rangle} q_{i}^{1+\left\langle i, \zeta^{\prime}\right\rangle}\left\{y_{1}, y_{2} \theta_{i}\right\}-\pi_{i}^{p\left(y_{1}\right)} q_{i}^{1+\left\langle i,\left|y_{1}\right|-i-\zeta^{\prime}\right\rangle}\left\{y_{1}, \theta_{i} y_{2}\right\} .
\end{aligned}
$$

Then we see that

$$
\begin{aligned}
\left\{y_{1}^{-} E_{i} 1_{\zeta^{\prime}}, y_{2}^{-} 1_{\zeta}\right\} & =\pi_{i}^{1+\left\langle i, \zeta^{\prime}\right\rangle} q_{i}^{1+\left\langle i, \zeta^{\prime}\right\rangle}\left\{y_{1}, y_{2} \theta_{i}\right\} \\
& =\pi^{1+\left\langle i, \zeta^{\prime}+i^{\prime}\right\rangle} q_{i}^{-1+\left\langle i, \zeta^{\prime}+i^{\prime}\right\rangle}\left\{y_{1}^{-} 1_{\zeta^{\prime}+i}, y_{2}^{-} F_{i} 1_{\zeta^{\prime}+i^{\prime}}\right\}
\end{aligned}
$$

which proves (a).

Proposition 5.29. We have $(\varrho(x), \varrho(y))=(x, y)$ for all $x, y \in \dot{\mathbf{U}}$.

Proof. It suffices to show that $x, y \mapsto\left(\varrho(x), \varrho^{\prime}(y)\right)$ satisfies the defining properties of $(-,-)$. All of these are obvious except Theorem $5.26(2)$. However, this follows from the previous proposition and the fact that $\varrho \tau_{1}=\bar{\tau}_{1} \varrho$.

Lemma 5.30. For $x, x^{\prime} \in \mathbf{f}_{\nu}, \nu \in N[I]$ and $\lambda \in X,\left(x^{+} 1_{\lambda}, x^{\prime+} 1_{\lambda}\right)=\pi_{\nu} \pi^{\langle\tilde{\nu}, \lambda\rangle}\left(x, x^{\prime}\right)$.

Proof. We have

$$
\begin{aligned}
\left(x^{+} 1_{\lambda}, x^{\prime+} 1_{\lambda}\right) & =\left(1_{\lambda}, \tau_{1}\left(x^{+}\right) x^{\prime+} 1_{\lambda}\right)=\left(1_{-\lambda}, 1_{-\lambda} \varrho\left(x^{\prime+}\right) \varrho \tau_{1}\left(x^{+}\right)\right) \\
& =\left(1_{-\lambda}, \varrho\left(x^{\prime+}\right) \bar{\tau}_{1} \varrho\left(x^{+}\right) 1_{-\lambda}\right) .
\end{aligned}
$$

Then we may rearrange to obtain

$$
\left(x^{+} 1_{\lambda}, x^{\prime+} 1_{\lambda}\right)=\left(\tau_{1} \varrho\left(x^{\prime+}\right) 1_{-\lambda}, \bar{\tau}_{1} \varrho\left(x^{+}\right) 1_{-\lambda}\right) .
$$

Now note that for any $x \in \mathbf{f}_{\nu}, \tau_{1} \varrho\left(x^{+}\right)=\pi_{\nu} q_{\nu} q^{\mathbf{N}(\nu)} \varrho(x)^{-} \tilde{J}_{\nu} \tilde{K}_{-\nu}$ where recall $\mathbf{N}\left(i_{1}+\right.$ $\left.\ldots+i_{n}\right)=\sum_{s<t} i_{s} \cdot i_{t}$. Since $\mathbf{N}(\nu) \in 2 \mathbb{Z}$ for all $\nu \in \mathbb{Z}[I]$, we obtain $\bar{\tau}_{1} \varrho\left(x^{+}\right)=$ $q_{-\nu} q^{-\mathbf{N}(\nu)} \varrho(x)^{-} \tilde{K}_{\nu}$. Then

$$
\left(\tau_{1} \varrho\left(x^{\prime+}\right) 1_{-\lambda}, \bar{\tau}_{1} \varrho\left(x^{+}\right) 1_{-\lambda}\right)=\pi_{\nu} \pi^{\langle\tilde{\nu}, \lambda\rangle}\left(x, x^{\prime}\right)
$$


Proposition 5.31. We have $\left(\omega(x), \omega^{-1}(y)\right)=(x, y)$ for all $x, y \in \dot{\mathbf{U}}$.

Proof. It suffices to show that $x, y \mapsto\left(\omega(x), \omega^{-1}(y)\right)$ satisfies the defining properties of $(-,-)$. It is clear that $(1)$ holds. Since $\omega \tau_{1}=\tau_{1} \omega$, it is clear that (2) is satisfied. Note that $\omega\left(x^{-} 1_{\lambda}\right)=\pi_{\nu} \pi^{\langle\tilde{\nu}, \lambda\rangle} x^{+}$, while $\omega^{-1}\left(x^{\prime-} 1_{\lambda}\right)=x^{\prime+} 1_{\lambda}$ for each $x, x^{\prime} \in \mathbf{f}_{\nu}$, whence (3) holds by the previous lemma. Finally, we note that $\omega^{2}(y)=\pi_{\nu} \pi^{\langle\tilde{\nu}, \lambda\rangle} x$ for any $x \in \dot{\mathbf{U}} 1_{\lambda}$, and hence $\left(\omega^{2}(x), \omega^{2}(y)\right)=(x, y)$ for any $x, y \in \dot{\mathbf{U}}$, proving (4).

Example 5.32. We compute the following inner products. Let $\lambda_{i}=\langle i, \lambda\rangle$.

$$
\begin{aligned}
& \left(F_{i}^{(k)} 1_{\lambda}, F_{i}^{(k)} 1_{\lambda}\right)=\pi^{\left(\begin{array}{c}
k \\
2
\end{array}\right)} \prod_{s=1}^{k} \frac{1}{1-\left(\pi_{i} q_{i}^{2}\right)^{s}} \\
& \left(E_{i}^{(k)} 1_{\lambda}, E_{i}^{(k)} 1_{\lambda}\right)=\pi_{i}^{\left(\begin{array}{c}
k+1 \\
2
\end{array}\right)+k \lambda_{i}} \prod_{s=1}^{k} \frac{1}{1-\left(\pi_{i} q_{i}^{2}\right)^{s}} \\
& \left(E_{i} F_{i} 1_{\lambda}, 1_{\lambda}\right)=\frac{q_{i}^{1-\lambda_{i}}}{1-\pi_{i} q_{i}^{2}} \\
& \left(E_{i} F_{i} 1_{\lambda}, E_{i} F_{i} 1_{\lambda}\right)=\pi^{\lambda_{i}-1} \frac{1+\left(\pi_{i} q_{i}^{2}\right)^{1-\lambda_{i}}}{\left(1-\pi_{i} q_{i}^{2}\right)^{2}} \\
& \left(E_{i} F_{i} 1_{\lambda}, F_{i} E_{i} 1_{\lambda}\right)=\pi^{\lambda_{i}} \frac{1+\pi_{i} q_{i}^{2}}{\left(1-\pi_{i} q_{i}^{2}\right)^{2}}
\end{aligned}
$$

We note that under the identification $v^{2}=\pi q^{2}$, these values are formally similar to the values of the analogous bilinear form on $\left.\dot{\mathbf{U}}\right|_{\pi=1}$ over $\mathbb{Q}(v)$ (but with an additional factor of some power of $\pi$ ).

Proposition 5.33. Let $\tau_{1}^{\prime}$ be the $\mathbb{Q}(q)^{\pi}$-linear automorphism of $\mathbf{U}$ defined by

$$
\tau_{1}^{\prime}\left(E_{i}\right)=q K F, \quad \tau_{1}^{\prime}\left(F_{i}\right)=q K^{-1} E, \quad \tau_{1}^{\prime}(K)=K, \quad \tau_{1}(J)=J
$$

Then there is a $\mathbb{Q}(q)^{\pi}$-bilinear pairing $(-,-): \dot{\mathbf{U}} \times \dot{\mathbf{U}} \rightarrow \mathbb{Q}(q)^{\pi}$ such that the following hold.

1. $\left(1_{\lambda_{1}} x 1_{\lambda_{2}}, 1_{\lambda_{1}^{\prime}} x^{\prime} 1_{\lambda_{2}^{\prime}}\right)$ is zero unless $\lambda_{1}=\lambda_{1}^{\prime}, \lambda_{2}=\lambda_{2}^{\prime}$; 
2. $(u x, y)=\left(x, \tau_{1}^{\prime}(u) y\right)$ for all $x, y \in \dot{\mathbf{U}}$ and $u \in \mathbf{U}$;

3. $\left(x^{-} 1_{\lambda}, x^{-} 1_{\lambda}\right)=\left(x, x^{\prime}\right)$ for all $x, x^{\prime} \in \mathbf{f}$ and all $\lambda$;

4. We have $(x, y)=(y, x)$.

Moreover, we have $\left(\omega(x), \omega^{-1}(y)\right)^{\prime}=(\varrho(x), \varrho(y))^{\prime}=(x, y)^{\prime}$.

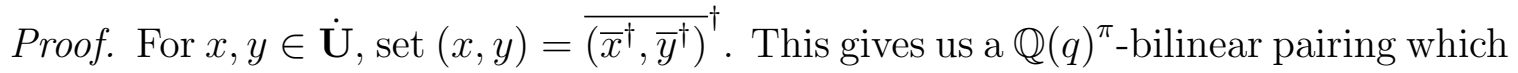
clearly satisfies (1) and (4). (2) follows from the observation that ${\overline{\tau_{1}}}^{\prime}\left(\bar{u}^{\dagger}\right)^{\dagger}=\tau_{1}(u)$.

For (3), by Theorem $5.26(3)$ we have $\left(x^{-} 1_{\lambda}, x^{\prime-} 1_{\lambda}\right)=\left(x^{\dagger}, x^{\prime \dagger}\right)^{\dagger}$. However, it is easy to check that the bilinear form $(-,-)^{\prime}: \mathbf{f} \times \mathbf{f} \rightarrow \mathbb{Q}(q)^{\pi}$ defined by $(x, y)^{\prime}=\left(x^{\dagger}, y^{\dagger}\right)^{\dagger}$ for $x, y \in \mathbf{f}$ satisfies the defining properties of $(-,-)$, hence $(x, y)^{\prime}=(x, y)$.

Lastly, we note that $\omega\left(\bar{u}^{\dagger}\right) \overline{\bar{\omega}^{-1}(u)}{ }^{\dagger}$ and $\varrho\left(\bar{u}^{\dagger}\right)={\overline{\left(\omega \varrho \omega^{-1}\right)(u)}}^{\dagger}$, so $\varrho$ - and $\omega$ invariance follows from the properties of $\{-,-\}$.

Remark 5.34. In [ClW], the bilinear form $(-,-)$ is defined on $\dot{\mathbf{U}}$ for $I=I_{\overline{1}}=$ $\{i\}$. With respect to this form, $\left(E^{(a)} 1_{n}, E^{(a)} 1_{n}\right)$ is not almost- $\pi$-orthonormal with respect to $q^{-1}$ in general (see Definition 5.37), a fact which is not desirable from a categorification standpoint. In this regard, the bilinear form defined in Theorem 5.26 is a better choice. However, in light of Remark 3.4, Proposition 5.33 demonstrates that $(-,-)$ is well suited to the $\dagger$-twisted $\dot{\mathbf{U}}$; that is, the algebra on generators $E^{\prime} 1_{n}$, $F^{\prime} 1_{n}$, and $1_{n}$ such that

$$
\begin{gathered}
1_{n} 1_{m}=\delta_{n m} 1_{n}, \quad 1_{n+2}\left(E^{\prime} 1_{n}\right) 1_{n}=E^{\prime} 1_{n}, \quad 1_{n-2}\left(F^{\prime} 1_{n}\right) 1_{n}=F^{\prime} 1_{n}, \\
E^{\prime} 1_{n-2} F^{\prime} 1_{n}-\pi F^{\prime} 1_{n+2} E^{\prime} 1_{n}=[n]^{\dagger} 1_{n}
\end{gathered}
$$

Let $\zeta \in X$ and $\lambda, \lambda^{\prime} \in X^{+}$such that $\lambda-\lambda^{\prime}=\zeta$. Let $(-,-)_{\lambda}$ denote the polarization on $V(\lambda)$, and let $(-,-)_{\lambda^{\prime}}$ denote the polarization on $V\left(\lambda^{\prime}\right)$. Then $(-,-)_{\lambda^{\prime}}$ can be viewed as a bilinear form on ${ }^{\omega} V\left(\lambda^{\prime}\right)$ and is in fact a polarization since $\omega$ and $\tau_{1}$ commute. 
Proposition 5.35. Consider the bilinear pairing $(-,-)_{\lambda, \lambda^{\prime}}: N\left(\lambda, \lambda^{\prime}\right) \times N\left(\lambda, \lambda^{\prime}\right) \rightarrow$ $\mathbb{Q}(q)^{\pi}$ defined by setting

$$
\left(x \otimes x^{\prime}, y \otimes y^{\prime}\right)_{\lambda, \lambda^{\prime}}=\pi_{\nu} \pi^{\left\langle\tilde{\nu}, \lambda^{\prime}\right\rangle}(x, y)_{\lambda}\left(x^{\prime}, y^{\prime}\right)_{\lambda^{\prime}}
$$

for homogeneous $x, x^{\prime} \in V(\lambda)$ and $y, y^{\prime} \in V\left(\lambda^{\prime}\right)$ and $y \in V\left(\lambda^{\prime}\right)_{\lambda-\nu^{\prime}}$ for some $\nu \in \mathbb{N}[I]$. Then $(-,-)_{\lambda \otimes \lambda^{\prime}}$ is a J-polarization.

Proof. This is an elementary verification, and we note that the $\pi_{\nu} \pi^{\left\langle\tilde{\nu}, \lambda^{\prime}\right\rangle}$ factor is related to Lemma 5.30.

Proposition 5.36. Let $x, y \in \dot{\mathbf{U}} 1_{\zeta}$. When the pair $\left(\lambda, \lambda^{\prime}\right)$ tends to $\infty$ (in the sense that $\langle i, \lambda\rangle$ and $\left\langle i, \lambda^{\prime}\right\rangle$ tends to $\infty$ for all $\left.i\right)$, the inner product $\left(x\left(\eta_{\lambda} \otimes \zeta_{-\lambda^{\prime}}\right), y\left(\eta_{\lambda} \otimes\right.\right.$ $\left.\left.\zeta_{-\lambda^{\prime}}\right)\right)_{\lambda, \lambda^{\prime}}$ converges in $\mathbb{Q}^{\pi}((q))$ to $\{x, y\}$.

Proof. Assume first that $x=x_{1}^{-} 1_{\zeta}$ and $y=y_{1}^{-} 1_{\zeta}$ for $x_{1}, y_{1} \in \mathbf{f}$ Then

$$
\begin{aligned}
& x\left(\eta_{\lambda} \otimes \zeta_{-\lambda^{\prime}}\right)=x_{1}^{-} \eta_{\lambda} \otimes \zeta_{-\lambda^{\prime}}, \\
& y\left(\eta_{\lambda} \otimes \zeta_{-\lambda^{\prime}}\right)=y_{1}^{-} \eta_{\lambda} \otimes \zeta_{-\lambda^{\prime}} .
\end{aligned}
$$

Therefore $\left(x\left(\eta_{\lambda} \otimes \zeta_{-\lambda^{\prime}}\right), y\left(\eta_{\lambda} \otimes \zeta_{-\lambda^{\prime}}\right)\right)_{\lambda, \lambda^{\prime}}=\left(x \eta_{\lambda}, y \eta_{\lambda}\right)_{\lambda}$, and the right-hand side converges to $(x, y)=\left\{x^{-} 1_{\zeta}, y^{-} 1_{\zeta}\right\}$ by Proposition 4.35 .

Now assume that $x=1_{\zeta}$ and $y$ is arbitrary. Then we may assume that $y=$ $\tau_{1}\left(x_{1}^{-}\right) y_{1}^{-} 1_{\zeta}$ for some $x_{1}, x_{2} \in \mathbf{f}$. But then by the polarization property,

$$
\left(1_{\zeta}\left(\eta_{\lambda} \otimes \zeta_{-\lambda^{\prime}}\right), y\left(\eta_{\lambda} \otimes \zeta_{-\lambda^{\prime}}\right)\right)_{\lambda, \lambda^{\prime}}=\left(x_{1}^{-}\left(\eta_{\lambda} \otimes \zeta_{-\lambda^{\prime}}\right), y_{1}^{-}\left(\eta_{\lambda} \otimes \zeta_{-\lambda^{\prime}}\right)\right)
$$

Using the previous case, this converges to

$$
\left\{x_{1}^{-} 1_{\zeta}, y_{1}^{-} 1_{\zeta}\right\}=\left\{1_{\zeta}, \tau_{1}\left(x_{1}^{-}\right) y_{1}^{-} 1_{\zeta}\right\}=\{x, y\}
$$

Finally, let us assume $x$ and $y$ are both arbitrary. We may assume that $x=u 1_{\zeta}$ for some $u \in \mathbf{U}$. Then

$$
\begin{aligned}
\left(x\left(\eta_{\lambda} \otimes \zeta_{-\lambda^{\prime}}\right), y\left(\eta_{\lambda} \otimes \zeta_{-\lambda^{\prime}}\right)\right)_{\lambda, \lambda^{\prime}} & =\left(u\left(\eta_{\lambda} \otimes \zeta_{-\lambda^{\prime}}\right), y\left(\eta_{\lambda} \otimes \zeta_{-\lambda^{\prime}}\right)\right)_{\lambda, \lambda^{\prime}} \\
& =\left(1_{\zeta}\left(\eta_{\lambda} \otimes \zeta_{-\lambda^{\prime}}\right), \tau_{1}(u) y\left(\eta_{\lambda} \otimes \zeta_{-\lambda^{\prime}}\right)\right) .
\end{aligned}
$$

Again, by the previous case, this converges to $\left\{1_{\zeta}, \tau_{1}(u) y\right\}=\{x, y\}$. 
Now we shall define a notion of almost-orthogonality with a view toward the $\pi$-orthonormality of our canonical basis of $\mathbf{f}$.

Definition 5.37. Given $\mathbb{Q}(q)^{\pi}$-modules $M, M^{\prime}$ and a pairing $(-,-): M \times M^{\prime} \rightarrow$ $\mathbb{Q}(q)^{\pi}$, we say a $\pi$-basis $B$ of $M$ is almost- $\pi$-orthonormal to a $\pi$-basis $B^{\prime}$ of $M^{\prime}$ if they satisfy the following conditions:

$$
\begin{gathered}
\left(b, b^{\prime}\right) \in \mathbb{Z}^{\pi}[[q]] \cap \mathbb{Q}(q)^{\pi} ; \\
\left(b, b^{\prime}\right) \in q \mathbb{Z}^{\pi}[[q]] \text { if } b \neq b^{\prime} \text { and } b \neq \pi b^{\prime} ; \\
(b, b) \in \pi^{\epsilon}+q \mathbb{Z}^{\pi}[[q]] \text { for some } \epsilon \in\{0,1\} .
\end{gathered}
$$

We note that by Lemma 4.31 , the canonical bases $\mathbb{B}$ and $\mathbb{B}(\lambda) \subset V(\lambda)$ are almost$\pi$-orthonormal.

Theorem 5.38. The basis $\dot{\mathbb{B}}$ is almost- $\pi$-orthonormal.

Proof. It is trivial that $\left(b \diamond_{\zeta} b^{\prime}, b_{1} \diamond_{\zeta^{\prime}} b_{1}^{\prime}\right)=0$ if $\zeta \neq \zeta^{\prime}$. Recall by construction and by Corollary 5.24 that for all $\lambda, \lambda^{\prime} \in X^{+}$such that $\lambda-\lambda^{\prime}=\zeta$,

$$
\begin{gathered}
\Delta\left(b \diamond_{\zeta} b^{\prime}\right) \eta_{\lambda} \otimes \xi_{-\lambda^{\prime}}=\left(b \diamond b^{\prime}\right)_{\lambda, \lambda^{\prime}}, \\
\Delta^{\prime}\left(b \zeta_{\zeta} b^{\prime}\right) \eta_{\lambda} \otimes \xi_{-\lambda^{\prime}}=\pi^{\epsilon}\left(b \diamond b^{\prime}\right)_{\lambda, \lambda^{\prime}},
\end{gathered}
$$

where $\epsilon \in\{0,1\}$ (and by convention, we set $\left(b \diamond_{s} b^{\prime}\right)_{\lambda, \lambda^{\prime}}=0$ if $b \notin \mathbb{B}(\lambda)$ or $\left.b^{\prime} \notin \mathbb{B}\left(\lambda^{\prime}\right)\right)$.

By the previous proposition, it suffices to show that for all $\lambda, \lambda^{\prime} \in X^{+}$such that if $\lambda-\lambda^{\prime}=\zeta$ such that $b \in B(\lambda)$ and $b^{\prime} \in B\left(\lambda^{\prime}\right),\left(b \diamond b^{\prime}\right)_{\lambda, \lambda^{\prime}}$ is almost- $\pi$-orthonormal to $\left(b_{1} \diamond^{\prime} b_{1}^{\prime}\right)_{\lambda, \lambda^{\prime}}$ for all $b_{1}, b_{1}^{\prime}$. But this follows from the definition of the bilinear form and the almost- $\pi$-orthonomality of $\mathbb{B}(\lambda)$.

Remark 5.39. It should be noted that, in contrast to the non-super case, a characterization of $\dot{\mathbb{B}}$ via almost- $\pi$-orthonormality is not possible, since in particular it is not possible for $\mathbb{B}$. 


\section{Appendix A}

\section{Twisted Bialgebras}

In this appendix, we will discuss the structure theory of a class of algebras related to $\mathbf{f}$, which we call twisted bialgebras. Our motivation for studying these algebras is to construct a framework for understanding the transformation between a one- and two-parameter half-quantum group which was defined by Fan-Li [FL]. In particular, this helps to explain the twistor defined in $\S 2.5$, which is a variant of Fan-Li's transformation.

We define twisted bialgebras as natural generalizations of a notion of twisted bialgebra already existing in the literature (cf. [LZ]) and we note it is also closely related to the constructions appearing in $[\mathrm{AYY}, \mathrm{FL}, \mathrm{Gr}, \mathrm{HP}]$. After defining these algebras, we develop some general structure theory à la Green $[\mathrm{Gr}]$. We then proceed to define a generalization of Fan-Li's transformation, which is a twisting of the multiplication on the algebra, and we show that the resulting bialgebra structure is essentially unique in some sense. In particular, we derive some algebra isomorphisms of which the twistor on $\mathbf{f}$ is a particular example.

\section{A.1 Definitions}

Let $\mathbb{K}$ be a field. Let BF be the space of $\mathbb{Z}$-valued bilinear forms on $\mathbb{Z}[I]$. Let $T$ be the the group of bicharacters; that is, the group of functions $f: \mathbb{Z}[I] \times \mathbb{Z}[I] \rightarrow \mathbb{K}^{\times}$under point-wise multiplication which is generated by the functions $f$ such that $f(\eta, \nu)=$ 
$x^{b(\eta, \nu)}$ for all $\eta, \nu \in \mathbb{Z}[I]$ and some $b \in \mathrm{BF}, x \in \mathbb{K}^{\times}$. In particular, each $f \in T$ satisfies $f(\mu, \eta+\nu)=f(\mu, \eta) f(\mu, \nu)$ and $f(\mu+\eta, \nu)=f(\mu, \nu) f(\eta, \nu)$. Let $\tau: T \rightarrow T$ be the automorphism where $\tau(f)(\eta, \mu)=f(\mu, \eta)$. The collection of $\tau$-invariant elements are called symmetric bicharacters, and the set of elements $f$ with $\tau(f)=f^{-1}$ will be called skew-symmetric bicharacters.

Let $t, t^{\prime} \in T$. We define $\mathbb{F}$-bialgebra $\mathrm{F}$ to be of type $\left(I, t, t^{\prime}\right)$ if it satisfies the following properties:

(TB1) $\mathrm{F}=\bigoplus_{\mu \in \mathbb{Z}[I]^{+}} F_{\mu}$ is $\mathbb{Z}[I]^{+}$-graded algebra such that $\mathrm{F}_{0}=\mathbb{F} 1, \mathrm{~F}_{i}=\mathbb{F} \theta_{i}$, and the elements $\theta_{i}$ generate $\mathrm{F}$.

(TB2) There is a comultiplication $r: F \rightarrow F \otimes F$ such that

(a) $r\left(\theta_{i}\right)=\theta_{i} \otimes 1+1 \otimes \theta_{i}$;

(b) $r$ is an algebra homomorphism where $\mathrm{F} \otimes \mathrm{F}$ is given the multiplication

$$
(x \otimes y)\left(x^{\prime} \otimes y^{\prime}\right)=t\left(|y|,\left|x^{\prime}\right|\right) x x^{\prime} \otimes y y^{\prime}
$$

(TB3) There is a $\mathbb{K}$-valued symmetric bilinear form $(-,-)$ on $\mathrm{F}$ such that

(a) $\left(\mathrm{F}_{\mu}, \mathrm{F}_{\nu}\right)=0$ if $\mu \neq \nu$;

(b) $(1,1)=1$ and $\left(\theta_{i}, \theta_{i}\right) \neq 0$;

(c) $(x y, z)=(x \otimes y, r(z))$, where $\left(x \otimes y, x^{\prime} \otimes y^{\prime}\right)=t^{\prime}\left(|y|,\left|x^{\prime}\right|\right)\left(x, x^{\prime}\right)\left(y, y^{\prime}\right)$.

We call $\mathrm{F}$ a twisted bi-algebra. If $\mathrm{G}$ is another twisted bialgebra of type $\left(I, s, s^{\prime}\right)$ with generators $\vartheta_{i}$, we will say $\mathrm{F}$ is isomorphic to $\mathrm{G}$ as twisted bi-algebras if there is an algebra isomorphism $\phi: \mathrm{F} \rightarrow \mathrm{G}$ such that $\phi\left(\theta_{i}\right)=\vartheta_{i}$.

Given a twisted bialgebra $\mathrm{F}$ of type $\left(I, t, t^{\prime}\right)$, then $\mathrm{F}^{\otimes n}=\mathrm{F} \otimes_{\mathbb{K}} \mathrm{F} \otimes_{\mathbb{K}} \ldots \otimes_{\mathbb{K}} \mathrm{F}$ has a natural algebra structure given by iterating (TB2)(b). It is an easy exercise to verify that $r$ is co-associative, and in particular that iterating $r$ (by applying inductively to any choice of tensor factor) induces an algebra homomorphism $r^{n}: \mathrm{F} \rightarrow \mathrm{F}^{\otimes n}$. 
Lemma A.1. An algebra of type $\left(I, t, t^{\prime}\right)$ exists if and only if $t^{\prime} t=\tau\left(t^{\prime} t\right)$; that is, $t t^{\prime}$ is symmetric.

Proof. Suppose $t, t^{\prime} \in T$ satisfy $t t^{\prime}=\tau\left(t t^{\prime}\right)$. Then we have

$$
t^{\prime}(\eta, \nu) t(\eta, \nu)=t^{\prime}(\nu, \eta) t(\nu, \eta) \text { for all } \eta, \mu \in \mathbb{Z}[I]
$$

The existence of an algebra satisfying (TB1)-(TB3) absent the bilinear form being symmetric is trivial. Indeed, the free algebra $\mathcal{F}$ on $I$ with the given algebra structure on $\mathcal{F}^{\otimes 2}$ satisfies (1) and (2), and multiplication on the restricted dual $\mathcal{F}^{*}$ is induced by $r$ with a twist by $t^{\prime}$ so that $f g=t^{\prime}(|g|,|f|)(f \otimes g) \circ r$. The natural algebra homomorphism $F \rightarrow F^{*}$ gives rise to a unique bilinear form satisfying (3a)-(3c). To show this form is symmetric, by uniqueness it is equivalent to proving that $\left(x, y^{\prime} y^{\prime \prime}\right)=$ $\left(r(x), y^{\prime} \otimes y^{\prime \prime}\right)$. This is trivially true when $x=\theta_{i}$.

Let $x=x^{\prime} x^{\prime \prime}$, and for $z \in\left\{x^{\prime}, x^{\prime \prime}, y^{\prime}, y^{\prime \prime}\right\}$ set $r(z)=\sum z_{1} \otimes z_{2}$. Then $r(x)=$ $\sum t\left(\left|x_{2}^{\prime}\right|,\left|x_{1}^{\prime \prime}\right|\right) x_{1}^{\prime} x_{1}^{\prime \prime} \otimes x_{2}^{\prime} x_{2}^{\prime \prime}$ and $r\left(y^{\prime} y^{\prime \prime}\right)=\sum t\left(\left|y_{2}^{\prime}\right|,\left|y_{1}^{\prime \prime}\right|\right) y_{1}^{\prime} y_{1}^{\prime \prime} \otimes y_{2}^{\prime} y_{2}^{\prime \prime}$. On one hand,

$$
\left(x^{\prime} x^{\prime \prime}, y^{\prime} y^{\prime \prime}\right)=\left(x^{\prime} \otimes x^{\prime \prime}, r\left(y^{\prime} y^{\prime \prime}\right)\right)=\sum t^{\prime}\left(\left|x^{\prime \prime}\right|,\left|y_{1}^{\prime} y_{1}^{\prime \prime}\right|\right) t\left(\left|y_{2}^{\prime}\right|,\left|y_{1}^{\prime \prime}\right|\right)\left(x^{\prime}, y_{1}^{\prime} y_{1}^{\prime \prime}\right)\left(x^{\prime \prime}, y_{2}^{\prime} y_{2}^{\prime \prime}\right)
$$

which by induction implies

$$
\begin{aligned}
\left(x^{\prime} x^{\prime \prime}, y^{\prime} y^{\prime \prime}\right)=\sum & t^{\prime}\left(\left|x^{\prime \prime}\right|,\left|y_{1}^{\prime} y_{1}^{\prime \prime}\right|\right) t\left(\left|y_{2}^{\prime}\right|,\left|y_{1}^{\prime \prime}\right|\right) t^{\prime}\left(\left|x_{2}^{\prime}\right|,\left|y_{1}^{\prime}\right|\right) t^{\prime}\left(\left|x_{2}^{\prime \prime}\right|,\left|y_{2}^{\prime}\right|\right) \\
& \times\left(x_{1}^{\prime}, y_{1}^{\prime}\right)\left(x_{2}^{\prime}, y_{1}^{\prime \prime}\right)\left(x_{1}^{\prime \prime}, y_{2}^{\prime}\right)\left(x_{2}^{\prime \prime}, y_{2}^{\prime \prime}\right) .
\end{aligned}
$$

On the other hand,

$$
\begin{gathered}
\left(r(x), y^{\prime} \otimes y^{\prime \prime}\right)=\sum t^{\prime}\left(\left|x_{2}^{\prime} x_{2}^{\prime \prime}\right|,\left|y^{\prime}\right|\right) t\left(\left|x_{2}^{\prime}\right|,\left|x_{1}^{\prime \prime}\right|\right)\left(x_{1}^{\prime} x_{1}^{\prime \prime}, y^{\prime}\right)\left(x_{2}^{\prime} x_{2}^{\prime \prime}, y^{\prime \prime}\right) \\
=\sum t^{\prime}\left(\left|x_{2}^{\prime} x_{2}^{\prime \prime}\right|,\left|y^{\prime}\right|\right) t\left(\left|x_{2}^{\prime}\right|,\left|x_{1}^{\prime \prime}\right|\right) t^{\prime}\left(\left|x_{1}^{\prime \prime}\right|,\left|y_{1}^{\prime}\right|\right) t^{\prime}\left(\left|x_{2}^{\prime \prime}\right|,\left|y_{1}^{\prime \prime}\right|\right) \\
\quad \times\left(x_{1}^{\prime}, y_{1}^{\prime}\right)\left(x_{1}^{\prime \prime}, y_{2}^{\prime}\right)\left(x_{2}^{\prime}, y_{1}^{\prime \prime}\right)\left(x_{2}^{\prime \prime}, y_{2}^{\prime \prime}\right) .
\end{gathered}
$$

Comparing these, we see they are equal if

$$
\begin{aligned}
& t^{\prime}\left(\left|x_{2}^{\prime} x_{2}^{\prime \prime}\right|,\left|y^{\prime}\right|\right) t\left(\left|x_{2}^{\prime}\right|,\left|x_{1}^{\prime \prime}\right|\right) t^{\prime}\left(\left|x_{1}^{\prime \prime}\right|,\left|y_{1}^{\prime}\right|\right) t^{\prime}\left(\left|x_{2}^{\prime \prime}\right|,\left|y_{1}^{\prime \prime}\right|\right) \\
& =t^{\prime}\left(\left|x^{\prime \prime}\right|,\left|y_{1}^{\prime} y_{1}^{\prime \prime}\right|\right) t\left(\left|y_{2}^{\prime}\right|,\left|y_{1}^{\prime \prime}\right|\right) t^{\prime}\left(\left|x_{2}^{\prime}\right|,\left|y_{1}^{\prime}\right|\right) t^{\prime}\left(\left|x_{2}^{\prime \prime}\right|,\left|y_{2}^{\prime}\right|\right) .
\end{aligned}
$$


Since the bilinear form is only nonzero between homogeneous elements of the same weight, this is equivalent to

$$
\begin{aligned}
& t^{\prime}\left(\mu_{2}+\nu_{2}, \mu_{1}+\nu_{1}\right) t\left(\mu_{2}, \nu_{1}\right) t^{\prime}\left(\nu_{1}, \mu_{1}\right) t^{\prime}\left(\nu_{2}, \mu_{2}\right) \\
& =t^{\prime}\left(\nu_{1}+\nu_{2}, \mu_{1}+\mu_{2}\right) t\left(\nu_{1}, \mu_{2}\right) t^{\prime}\left(\mu_{2}, \mu_{1}\right) t^{\prime}\left(\nu_{2}, \nu_{1}\right)
\end{aligned}
$$

where $\left|x_{1}^{\prime}\right|=\left|y_{1}^{\prime}\right|=\mu_{1},\left|x_{2}^{\prime}\right|=\left|y_{1}^{\prime \prime}\right|=\mu_{2},\left|x_{1}^{\prime \prime}\right|=\left|y_{2}^{\prime}\right|=\nu_{1}$, and $\left|x_{2}^{\prime \prime}\right|=\left|y_{2}^{\prime \prime}\right|=\nu_{2}$. By expanding and rearranging factors, we see that $(\star)$ is equivalent to (A.1), which shows that we get such a symmetric bilinear form (and hence a twisted bialgebra) when (A.1) holds.

On the other hand, if $\mathrm{F}$ is a twisted bialgebra of type $\left(I, t, t^{\prime}\right)$, then (TB3) implies (A.1) by checking on the generators. To wit,

$\left(\theta_{i} \theta_{j}, \theta_{j} \theta_{i}\right)=\left(\theta_{i} \otimes \theta_{j}, \theta_{j} \theta_{i} \otimes 1+\theta_{j} \otimes \theta_{i}+t(j, i) \theta_{i} \otimes \theta_{j}+1 \otimes \theta_{j} \theta_{i}\right)=t(j, i) t^{\prime}(j, i)\left(\theta_{j}, \theta_{j}\right)\left(\theta_{i}, \theta_{i}\right)$

hence by symmetry and the condition $(3 \mathrm{~b})$, we must have $t(j, i) t^{\prime}(j, i)=t(i, j) t^{\prime}(i, j)$ for all $i, j$.

We note the following immediate corollary.

Corollary A.2. Suppose that $s, t, t^{\prime} \in T$ such that $s$ and $t t^{\prime}$ are symmetric. Then there exist twisted bialgebras of types $(I, s, 1),\left(I, t s, t^{\prime}\right),\left(I, t, t^{\prime} s\right)$, and $(I, t, \tau(t))$.

Remark A.3. We shall see later that there is a unique non-degenerate algebra only depending on $I, t$, and the coset $t^{\prime} S$, where $S$ is the subgroup of symmetric bicharacters.

Let $\mathrm{F}$ be a bialgebra of type $\left(I, t, t^{\prime}\right)$. Let $\langle I\rangle$ denote the words in $I$, and for $w=i_{1} \ldots i_{\ell} \in\langle I\rangle$ let $\theta_{w}=\theta_{i_{1}} \ldots \theta_{i_{\ell}}$.

Let $\underline{\ell}=\{1, \ldots, \ell\}$ and $\mathbf{P}=\left\{P_{1}, \ldots, P_{n}\right\}$ be a partition of $\underline{\ell}$ into $n$ (possibly empty) sets. Then for $x, y \in \underline{\ell}$, we say $x<_{\mathbf{P}} y$ if $x \in P_{a}$ and $y \in P_{b}$ such that $x<y$ and $a>b$. Then we define

$$
t(w, \mathbf{P})=\prod_{x<\mathbf{P} y} t\left(i_{x}, i_{y}\right)
$$


In the case where $n=\ell$ and no set in $\mathbf{P}$ is empty, the partition defines a unique permutation $s_{\mathbf{P}} \in S_{\ell}$ via $s_{\mathbf{P}}(a)=b$ if $b \in P_{a}$, and conversely any permutation $s \in S_{\ell}$ defines a unique partition $\mathbf{P}_{s}$. In this case, we will write $t(w, s)=t\left(w, \mathbf{P}_{s}\right)$.

Lemma A.4. Let $w=i_{1} \ldots i_{\ell} \in\langle I\rangle$ of length $\ell$ and let $n \geq 1$. Then

$$
r^{(n)}\left(\theta_{w}\right)=\sum_{\mathbf{P}} t(w, \mathbf{P}) \theta_{w_{1}} \otimes \ldots \otimes \theta_{w_{n+1}}
$$

where the sum is over $(n+1)$-set partitions $\mathbf{P}=\left\{P_{1}, \ldots, P_{a+1}\right\}$ of $\underline{\ell}$ and $w_{b}=$ $i_{p_{1}} i_{p_{2}} \ldots i_{p_{c}}$ with $P_{b}=\left\{p_{1}<\ldots<p_{c}\right\}$.

Proof. Note that $r^{(n)}\left(\theta_{i}\right)=\sum_{k} 1^{\otimes k} \otimes \theta_{i} \otimes 1^{\otimes n-1-k}$ so this is trivially true on generators. The proof may be finished by induction in a straightforward way, which is left as an exercise to the reader.

As in the proof of Lemma A.1, the free algebra $\mathcal{F}$ can be made a twisted bialgebra of type $\left(I, t, t^{\prime}\right)$ satisfying (A.1), and so its coproduct $r$ induces a product defined on its dual $\mathcal{F}^{*}$. Let $\mathcal{G}$ be the subalgebra of $\mathcal{F}^{*}$ generated by the elements $\xi_{w}$ with $w \in\langle I\rangle$ such that $\xi_{w}\left(\theta_{v}\right)=\delta_{v w}$ for all $v \in\langle I\rangle$. First we will express the product in the $\xi$-basis. Note that $\xi_{i_{1}} \ldots \xi_{i_{\ell}}=\tau\left(t^{\prime}\right)\left(w, \omega_{\ell}\right) \xi_{i_{1}} \otimes \ldots \otimes \xi i_{\ell} \circ r^{(l-1)}$, where $\omega_{\ell}$ is the order-reversing permutation on $\underline{\ell}$. For convenience, let us use $t^{\prime}(w)=\tau\left(t^{\prime}\right)\left(w, \omega_{\ell}\right)$. Note that if $t^{\prime}$ is symmetric, then $t^{\prime}(w)=t^{\prime}(v)$ when $|w|=|v|$ and we write $t^{\prime}(\nu)=t^{\prime}(w)$, but in general this is not the case.

Example A.5. Let $i, j \in I$. Then $t^{\prime}(i j)=t^{\prime}(j, i)$ and $t^{\prime}(j i)=t^{\prime}(i, j)$ hence $t^{\prime}(w)$ only depends on $|w|$ if and only if $t^{\prime}$ is symmetric.

Let $w=i_{1} \ldots i_{\ell} \in\langle I\rangle$ and let $v \in\langle I\rangle$. Then using Lemma A.4 and the definition of the product, we see that $\xi_{i_{1}} \ldots \xi_{i_{\ell}}\left(\theta_{v}\right)=0$ if $|v| \neq|w|$. On the other hand, if $|v|=|w|$, then $\xi_{i_{1}} \otimes \ldots \otimes \xi_{i_{\ell}}$ evaluates to 0 on all the summands of $r^{(\ell-1)}$ in (A.3) except if, for some $s \in S_{\ell}, P_{a}=\{s(a)\}$ with $j_{s(a)}=i_{a}$ for all $a$, yielding $\xi_{i_{1}} \otimes \ldots \otimes \xi_{i_{\ell}}\left(\theta_{v}\right)=t(w, s)$. Define the action of $S_{\ell}$ on $\ell$-length words via $s\left(k_{1} \ldots k_{\ell}\right)=k_{s(1)} \ldots k_{s(\ell)}$. Then we obtain the following lemma. 
Lemma A.6. Let $w=i_{1} \ldots i_{\ell} \in\langle I\rangle$. Then $\xi_{i_{1}} \ldots \xi_{i_{\ell}}=t^{\prime}(w) \sum_{s \in S_{\ell}} t(w, s) \xi_{s^{-1}(w)}$.

We call this product the twisted shuffle product on $\mathcal{G}$. It can be generalized to an arbitrary collection of words rather than letters, though we will not do this here.

Define $M_{w, v}(t)=\sum_{v=s^{-1}(w)} t(w, s)$, so that $\xi_{i_{1}} \ldots \xi_{i_{\ell}}=t^{\prime}(w) \sum_{v} M_{w, v}(t) \xi_{v}$. Note that the $M_{w, v}(t)$ only depend on the words $v, w \in\langle I\rangle$ and the bilinear forms comprising $t$. Specifically, suppose $t(\nu, \mu)=\prod_{n=1}^{m} c_{n}^{b_{n}(\nu, \mu)}$ for $c_{n} \in \mathbb{K}^{\times}$and $b_{n} \in \mathrm{BF}$, and define $t_{x}(\nu, \mu)=\prod_{n=1}^{m} x_{n}^{b_{n}(\nu, \mu)} \in \mathbb{Z}\left[x_{1}^{ \pm 1}, \ldots, x_{m}^{ \pm 1}\right]$. Then the $M_{w, v}\left(t_{x}\right)$ are polynomials which only depend on the bilinear forms $b_{n}$, and $M_{w, v}\left(t_{x}\right)\left(c_{1}, \ldots, c_{m}\right)=M_{w, v}(t)$.

Proposition A.7. Let $\mathrm{F}$ be a twisted bialgebra of type $\left(I, t, t^{\prime}\right)$. Then for $w=$ $i_{1} \ldots i_{\ell}, v \in\langle I\rangle$ of weight $\nu=i_{1}+\ldots+i_{\ell}$,

$$
\left(\theta_{w}, \theta_{v}\right)=t^{\prime}(w) M_{w, v}(t) B_{\nu}(\mathrm{F})
$$

where $B_{\nu}(\mathrm{F})=\prod_{a=1}^{\ell}\left(\theta_{i_{a}}, \theta_{i_{a}}\right)$.

Proof. By noting that $M_{i, i}(t)=1$ and $M_{w, v}(t)=0$ if $|w| \neq|v|$, clearly this holds for the generators. Now we may assume $w=i_{1} \ldots i_{\ell}$ with $\ell>1$. Then writing $r^{(\ell-1)}\left(\theta_{v}\right)=\sum_{\mathbf{P}} t(v, \mathbf{P}) \theta_{v_{1}} \otimes \ldots \otimes \theta_{v_{\ell}}$

$$
\begin{aligned}
\left(\theta_{w}, \theta_{v}\right) & =\left(\theta_{i_{1}} \otimes \ldots \otimes \theta_{i_{\ell}}, r^{(\ell-1)}\left(\theta_{v}\right)\right) \\
& =\sum_{\mathbf{P}} t(v, \mathbf{P}) t^{\prime}\left(i_{2},\left|v_{1}\right|\right) t^{\prime}\left(i_{3},\left|v_{1}\right|+\left|v_{2}\right|\right) \ldots t^{\prime}\left(i_{\ell},|v|-\left|v_{\ell}\right|\right)\left(\theta_{i_{1}}, \theta_{v_{1}}\right) \ldots\left(\theta_{i_{\ell}}, \theta_{v_{\ell}}\right) .
\end{aligned}
$$

Note that we must have $v_{a}=i_{a}$ for all $a$ to have a nonzero summand. This implies that the sum is only over partitions corresponding to permutations, the factors involving $t^{\prime}$ can be simplified to $t^{\prime}(w)$, and the factors involving inner products combine into a factor of $B_{\nu}(\mathrm{F})$. Therefore,

$$
\left(\theta_{w}, \theta_{v}\right)=t^{\prime}(w) B_{\nu}(\mathrm{F}) \sum_{s^{-1}(v)=w} t(w, s)=t^{\prime}(w) M_{w, v}(t) B_{\nu}(\mathrm{F}) .
$$

The following corollary is now obvious. 
Corollary A.8. Let $\mathrm{F}$ be a twisted bialgebra of type $\left(I, t, t^{\prime}\right)$ with coproduct $r$ and bilinear form $(-,-)$. Let $s \in T$ be symmetric. Then $\mathrm{F}$ is a twisted bialgebra of type $\left(I, t, s t^{\prime}\right)$ with respect to the coproduct $r$ and the bilinear form $(x, y)_{s}=s(|x|)(x, y)$.

The importance of the polynomials $M_{w, v}$ is made clear by the following proposition.

Proposition A.9. Let $x \in \mathrm{F}$, and write $x=\sum c_{w} \theta_{w}$. Then $x \in \operatorname{Rad}(-,-)$ if and only if

$$
\sum_{w \in\langle I\rangle_{\nu}} c_{w} t^{\prime}(w) M_{w, v}(t)=0
$$

for all $v \in\langle I\rangle$ and $\nu \in \mathbb{Z}[I]$. If in addition $t^{\prime}$ is symmetric, then $x \in \operatorname{Rad}(-,-)$ if and only if

$$
\sum_{w \in\langle I\rangle_{\nu}} c_{w} M_{w, v}(t)=0
$$

for all $v \in\langle I\rangle$ and $\nu \in \mathbb{Z}[I]$.

Proof. If $x$ is in the radical if and only if $\left(x, \theta_{v}\right)=0$ for all $v \in\langle I\rangle$. In particular, if $|v|=\nu\left(x, \theta_{v}\right)=0$ if and only if $\sum_{w \in\langle I\rangle} c_{w} M_{w, v}(\mathbf{x}) t^{\prime}(w) B_{\nu}(\mathrm{F})=0$ for all $v \in\langle I\rangle$ and $\nu \in \mathbb{Z}[I]$. Since $B_{\nu}(\mathrm{F})$ is invertible and $M_{w, v}=0$ if $|v| \neq|w|,\left(x, \theta_{v}\right)=0$ if and only if $\sum_{w \in\langle I\rangle_{\nu}} c_{w} M_{w, v}(\mathbf{x}) t^{\prime}(w)=0$ for all $v \in\langle I\rangle$ and $\nu \in \mathbb{Z}[I]$. In the case $t^{\prime}$ is symmetric, $t^{\prime}(w)$ only depends on $|w|=\nu$. Since $t^{\prime}(w)$ is invertible, it can be canceled, finishing the proof.

Corollary A.10. Let $\mathrm{F}$ and $\mathrm{G}$ be non-degenerate bi-algebras of types $\left(I, t, t^{\prime}\right)$ and $\left(I, t, t^{\prime \prime}\right)$ such that $t^{\prime} t^{\prime \prime-1}$ is symmetric. Then $\mathrm{F}$ and $\mathrm{G}$ are isomorphic as twisted bialgebras.

Proof. Write $t^{\prime}=s t^{\prime \prime}$ with $s$ a symmetric bicharacter. Denote the generators of $\mathrm{F}$ by $\theta$ and the generators of $\mathrm{G}$ by $\vartheta$. Then we have $\sum c_{w} \theta_{w}=0$ if and only if $\sum_{w \in\langle I\rangle_{\nu}} c_{w} t^{\prime}(w) M_{w, v}(t)=0$ for all $v \in\langle I\rangle$ and $\nu \in \mathbb{Z}[I]$ if and only if

$$
s(\nu) \sum_{w \in\langle I\rangle_{\nu}} c_{w} t^{\prime \prime}(w) M_{w, v}(t)=0
$$


for all $v \in\langle I\rangle$ and $\nu \in \mathbb{Z}[I]$ if and only if $\sum c_{w} \vartheta_{w}=0$ by Proposition A.9. Therefore, we have a well-defined bijection $\sum c_{w} \theta_{w} \mapsto \sum c_{w} \vartheta_{w}$.

\section{A.2 The Fan-Li transformation}

Let $\mathrm{F}$ be an algebra of type $\left(I, t, t^{\prime}\right)$ and let $s, s^{\prime} \in T$. Define

$$
\begin{gathered}
x * y=s^{\prime}(|x|,|y|) x y, \quad(x \otimes y) *\left(x^{\prime} \otimes y^{\prime}\right)=t(|y|,|x|) s(|y|,|x|)\left(x * x^{\prime}\right) \otimes\left(y * y^{\prime}\right), \\
r_{s}(x)=\sum s^{\prime}\left(\left|x_{1}\right|,\left|x_{2}\right|\right)^{-1} x_{1} \otimes x_{2} \text { where } r(x)=\sum x_{1} \otimes x_{2} .
\end{gathered}
$$

Write $r(x)=\sum x_{1} \otimes x_{2}$ and $r(y)=\sum y_{1} \otimes y_{2}$. Then

$$
\begin{gathered}
r_{s}(x) * r_{s}(y)=\sum s^{\prime}\left(\left|x_{1}\right|,\left|x_{2}\right|\right)^{-1} s^{\prime}\left(\left|y_{1}\right|,\left|y_{2}\right|\right)^{-1} s^{\prime}\left(\left|x_{1}\right|,\left|y_{1}\right|\right) s^{\prime}\left(\left|x_{2}\right|,\left|y_{2}\right|\right) \\
\times t\left(\left|x_{2}\right|,\left|y_{1}\right|\right) s\left(\left|x_{2}\right|,\left|y_{1}\right|\right) x_{1} y_{1} \otimes x_{2} y_{2}
\end{gathered}
$$

and

$$
r_{s}(x * y)=s^{\prime}(|x|,|y|) \sum s^{\prime}\left(\left|x_{1} y_{1}\right|,\left|x_{2} y_{2}\right|\right)^{-1} t\left(\left|x_{2}\right|,\left|y_{1}\right|\right) x_{1} y_{1} \otimes x_{2} y_{2}
$$

Comparing these equations, we see that $r_{s}(x) * r_{s}(y)=r_{s}(x * y)$ if

$$
\begin{gathered}
s^{\prime}(|x|,|y|) s^{\prime}\left(\left|x_{1} y_{1}\right|,\left|x_{2} y_{2}\right|\right)^{-1}=s^{\prime}\left(\left|x_{1}\right|,\left|x_{2}\right|\right)^{-1} s^{\prime}\left(\left|y_{1}\right|,\left|y_{2}\right|\right)^{-1} s^{\prime}\left(\left|x_{1}\right|,\left|y_{1}\right|\right) \\
\times s^{\prime}\left(\left|x_{2}\right|,\left|y_{2}\right|\right) s\left(\left|x_{2}\right|,\left|y_{1}\right|\right) .
\end{gathered}
$$

After to expanding and rearranging factors in $(\star)$, we see it is equivalent to

$$
s\left(\left|x_{2}\right|,\left|y_{1}\right|\right)=s^{\prime}\left(\left|x_{2}\right|,\left|y_{1}\right|\right) s^{\prime}\left(\left|y_{1}\right|,\left|x_{2}\right|\right)^{-1} .
$$

Now define

$$
(x, y)_{s}=(x, y), \quad\left(x \otimes y, x^{\prime} \otimes y^{\prime}\right)_{s}=\left(t^{\prime} \tau\left(s^{\prime 2}\right)\right)\left(|y|,\left|x^{\prime}\right|\right)\left(x, x^{\prime}\right)\left(y, y^{\prime}\right)
$$


Then we claim that $(x * y, z)_{s}=\left(x \otimes y, r_{s}(z)\right)_{s}$. Indeed if we write $r(z)=\sum z_{1} \otimes z_{2}$, then

$$
\begin{aligned}
\left(x \otimes y, r_{s}(z)\right)_{s} & =t^{\prime}\left(|y|,\left|z_{1}\right|\right) s^{\prime}\left(\left|z_{1}\right|,|y|\right) \sum s^{\prime}\left(\left|z_{1}\right|,\left|z_{2}\right|\right)^{-1}\left(x, z_{1}\right)\left(y, z_{2}\right) \\
& =\sum t^{\prime}\left(|y|,\left|z_{1}\right|\right) s^{\prime 2}(|x|,|y|) s^{\prime}(|x|,|y|)^{-1}\left(x, z_{1}\right)\left(y, z_{2}\right) \\
& =s^{\prime}(|x|,|y|)(x y, z)=(x * y, z)_{s}
\end{aligned}
$$

In particular, this bilinear form is symmetric, and hence $(\mathrm{F}, *)$ is a bialgebra of type $\left(I, t s, t^{\prime} \tau\left(s^{2}\right)\right)$. Hence we obtain the following.

Proposition A.11. Let $\mathrm{F}$ be a bialgebra of type $\left(I, t, t^{\prime}\right)$. Choose $s^{\prime} \in T$, and set $s=s^{\prime} \tau\left(s^{\prime}\right)^{-1}$. Let $*, r_{s},(-,-)_{s}$ be defined as in (A.4)-(A.6). Then $(\mathrm{F}, *)$ is a bialgebra of type $\left(I, t s, t^{\prime} \tau\left(s^{\prime}\right)^{2}\right)$ with coproduct $r_{s}$ and bilinear form $(-,-)_{s}$.

Corollary A.12. If $\mathrm{F}$ is the non-degenerate twisted bialgebra of type $\left(I, t, t^{\prime}\right)$, then $(\mathrm{F}, *)$ is isomorphic to the non-degenerate twisted bialgebra of type $\left(I, t s, t^{\prime} \tau\left(s^{\prime}\right)^{2}\right)$.

We will call $*$ the $s^{\prime}$-twisted multiplication and $(\mathrm{F}, *)$ the $s^{\prime}$-twist of $\mathrm{F}$. It turns out that the formulae for $r_{s}$ and $(-,-)_{s}$ are forced upon us by the axioms of twisted bialgebras. To wit, let $\mathrm{F}$ be a twisted bialgebra of type $\left(I, t, t^{\prime}\right)$ and $a, b, c, d, e \in T$. Define

$$
\begin{gathered}
x * y=a(|x|,|y|) x y \\
r_{b}(x)=\sum b\left(\left|x_{1}\right|,\left|x_{2}\right|\right) x_{1} \otimes x_{2} ; \\
(x \otimes y) *\left(x^{\prime} \otimes y^{\prime}\right)=(t c)\left(|y|,\left|x^{\prime}\right|\right)\left(x * x^{\prime}\right) \otimes\left(y * y^{\prime}\right) ; \\
(x, y)_{d}=d(|x|,|y|)(x, y) ; \\
\left(x \otimes y, x^{\prime} \otimes y^{\prime}\right)_{e}=\left(t^{\prime} e\right)\left(|y|,\left|x^{\prime}\right|\right)\left(x, x^{\prime}\right)_{d}\left(y, y^{\prime}\right)_{d} .
\end{gathered}
$$

Lemma A.13. Keep the notations of the previous paragraph. We have the following statements. 
1. $r_{b}$ is an algebra homomorphism with respect to $*$ if and only if $a=b^{-1}$ and $c=a \tau(a)^{-1}$.

2. We have $(x * y, z)_{d}=\left(x \otimes y, r_{b}(z)\right)_{e}$ if and only if $e=d \tau(d) \tau(a)^{2}$ if and only if $(x, y * z)_{d}=\left(r_{b}(x), y \otimes z\right)_{e}$.

In particular, $(F, *)$ is the a-twist of $\mathrm{F}$.

Proof. First, consider (1). Write $r(x)=\sum x_{1} \otimes x_{2}$ and $r(y)=\sum y_{1} \otimes y_{2}$. Then $r(x y)=\sum t\left(\left|x_{2}\right|,\left|y_{1}\right|\right) x_{1} y_{1} \otimes x_{2} y_{2}$, and by direct computation

$$
\begin{aligned}
& r_{b}(x) * r_{b}(y)=\sum b\left(\left|x_{1}\right|,\left|x_{2}\right|\right) b\left(\left|y_{1}\right|,\left|y_{2}\right|\right)(t c)\left(\left|x_{2}\right|,\left|y_{1}\right|\right) \\
& \times a\left(\left|x_{1}\right|,\left|y_{1}\right|\right) a\left(\left|x_{2}\right|,\left|y_{2}\right|\right) x_{1} y_{1} \otimes x_{2} y_{2}, \\
& r_{b}(x * y)=a(|x|,|y|) \sum b\left(\left|x_{1} y_{1}\right|,\left|x_{2} y_{2}\right|\right) x_{1} y_{1} \otimes x_{2} y_{2} .
\end{aligned}
$$

Therefore, we see that $r_{b}(x) * r_{b}(y)=r_{b}(x * y)$ if

$$
(t c)\left(\left|x_{2}\right|,\left|y_{1}\right|\right) a\left(\left|x_{1}\right|,\left|y_{1}\right|\right) a\left(\left|x_{2}\right|,\left|y_{2}\right|\right)=a(|x|,|y|) b\left(\left|y_{1}\right|,\left|x_{2}\right|\right) b\left(\left|x_{1}\right|,\left|y_{2}\right|\right) .
$$

If we write $\left|x_{2}\right|=\mu,\left|y_{1}\right|=\nu,\left|x_{1}\right|=\eta, y_{2}=\zeta$, then after rearranging ( $\star$ ) we see it is equivalent to

$$
c(\mu, \nu) a(\mu, \nu)^{-1} b(\nu, \mu)^{-1}=a(\eta, \zeta) b(\eta, \zeta) .
$$

Therefore, $(\star)$ is true if $a=b^{-1}$ and $c=a \tau(a)^{-1}$. On the other hand, suppose $x=\theta_{i}$ and $t=\theta_{j}$. Then the same computation yields

$$
\begin{gathered}
r_{s}\left(\theta_{i} * \theta_{j}\right)=a(i, j)\left(\theta_{i} \theta_{j} \otimes 1+b(i, j) \theta_{i} \otimes \theta_{j}+b(j, i) t(i, j) \theta_{j} \otimes \theta_{i}+1 \otimes \theta_{i} \theta_{j}\right), \\
r_{s}\left(\theta_{i}\right) * r_{s}\left(\theta_{j}\right)=a(i, j) \theta_{i} \theta_{j} \otimes 1+\theta_{i} \otimes \theta_{j}+t(i, j) c(i, j) \theta_{j} \otimes \theta_{i}+a(i, j) 1 \otimes \theta_{i} \theta_{j} .
\end{gathered}
$$

All of the summands are linearly independent, hence we obtain $a(i, j) b(i, j)=1$ and $a(i, j) b(j, i)=c(i, j)$. This finishes the proof of $(1)$.

The proof of (2) is similar, so to minimize tedious calculation we will restrict ourselves to the proof that $e=d \tau(d) \tau(a)^{2}$ is necessary for $(x * y, z)_{d}=\left(x \otimes y, r_{b}(z)\right)_{e}$ to hold. By direct computation,

$$
\left(\theta_{i} * \theta_{j}, \theta_{j} * \theta_{i}\right)_{d}=a(i, j) a(j, i) d(i+j, i+j) t(i, j) t^{\prime}(i, j)\left(\theta_{i}, \theta_{i}\right)\left(\theta_{j}, \theta_{j}\right),
$$


$\left(\theta_{i} \otimes \theta_{j}, r_{b}\left(\theta_{i} * \theta_{j}\right)\right)=\left(\theta_{i} \otimes \theta_{j}, t(j, i) c(j, i) \theta_{i} \otimes \theta_{j}\right)_{e}=\left(e t^{\prime} t c\right)(j, i) d(i, i) d(j, j)\left(\theta_{i}, \theta_{i}\right)\left(\theta_{j}, \theta_{j}\right)$.

By assumption, these inner products are equal. Moreover, by (TB3)(b), we have $\left(\theta_{i}, \theta_{i}\right)\left(\theta_{j}, \theta_{j}\right) \neq 0$, so

$$
\left(e t^{\prime} t c\right)(j, i) d(i, i) d(j, j)=a(i, j) a(j, i) d(i+j, i+j) t(i, j) t^{\prime}(i, j) .
$$

Expanding and rearranging the factors, we have the identity

$$
e(j, i)=a(i, j) a(j, i) c(j, i) d(i, j) d(j, i)=a(i, j)^{2} d(i, j) d(j, i) .
$$

Finally, we note that by Corollary A.8, $(\mathrm{F}, *)$ is a twisted bialgebra of type $\left(I, \operatorname{ta} \tau(a)^{-1}, t^{\prime} \tau(a)^{2}\right)$ which is exactly the $a$-twist of $\mathrm{F}$. .

Remark A.14. Note that the twist transformation defines a T-action on the class of twisted bialgebras. This transformation generalizes the twistor isomorphism in §2.5, as well as the connection between Lusztig's algebra $\mathbf{f}$ and the two-parameter version appearing in $[F L]$. 


\section{Bibliography}

[AYY] S. Azam, H. Yamane, and M. Yousofzadeh, Classification of Finite Dimensional Irreducible Representations of Generalized Quantum Groups via Weyl Groupoids, arXiv:1105.0160

[BW] H. Bao, W. Wang, Canonical bases in tensor products revisited, arXiv:1403.0039.

[BKK] G. Benkart, S.-J. Kang and M. Kashiwara, Crystal bases for the quantum superalgebra $U_{q}(\mathfrak{g l}(m, n))$, J. Amer. Math. Soc. 13 (2000), 295-331.

[BKM] G. Benkart, S.-J. Kang and D. Melville, Quantized enveloping algebras for Borcherds superalgebras, Trans. AMS. 350 (1998), 3297-3319.

[BLM] A.A. Beilinson, G. Lusztig, R. McPherson, A geometric setting for the quantum deformation of $\mathrm{GL}_{n}$, Duke Math. J. 61 (1990), 655-677.

[BK] J. Brundan and A. Kleshchev, Blocks of cyclotomic Hecke algebras and Khovanov-Lauda algebras, Invent. Math. 178 (2009), 451-484.

[ChW] S.-J. Cheng and W. Wang, Dualities and Representations of Lie Superalgebras, Graduate Studies in Mathematics 144, AMS, Providence, RI, 2002.

[C] S. Clark, Quantum supergroups IV. The modified form, arXiv:1312.4855.

[CFLW] S. Clark, Z. Fan, Y. Li and W. Wang, Quantum supergroups III. Twistors, accepted in Comm. Math. Phys., arXiv:1307.7056. 
[CHW1] S. Clark, D. Hill and W. Wang, Quantum supergroups I. Foundations, Transform. Groups 18 (2013), 1019-1053.

[CHW2] S. Clark, D. Hill and W. Wang, Quantum supergroups II. Canonical basis, arXiv:1304.7837.

[CHW3] S. Clark, D. Hill and W. Wang, Quantum shuffles and quantum supergroups of basic type, arXiv:1310.7523.

[ClW] S. Clark and W. Wang, Canonical basis for quantum osp(1|2), Lett. Math. Phys. 103 (2013), 207-231.

[D] V. Drinfeld, Quantum groups, Proceedings of the ICM (Berkeley, 1986), 798-820, Amer. Math. Soc., Providence, RI, 1987.

[EKL] A. Ellis, M. Khovanov and A. Lauda, The odd nilHecke algebra and its diagrammatics, IMRN, 2013, arXiv:1111.1320.

[EL] A. Ellis and A. Lauda, An odd categorification of $U_{q}\left(\mathfrak{s l}_{2}\right)$, arXiv:1307.7816.

[FL] Z. Fan and Y. Li, Two-parameter quantum algebras, canonical bases and categorifications, arXiv:1303.2429.

[Je] K. Jeong, Crystal bases for Kac-Moody superalgebras, J. of Alg. 237 (2002), $562-590$.

[Ji] M. Jimbo, A q-analogue of $U(\mathfrak{g l}(N+1))$, Hecke algebra, and the Yang-Baxter equation, Lett. Math. Phys. 11 (1986), 247-252.

[Kac] V. Kac, Lie Superalgebras, Adv. Math. 26 (1977), 8-96.

[Kas1] M. Kashiwara, On crystal bases of the Q-analogue of universal enveloping algebras, Duke Math. J. 63 (1991), 456-516. 
[Kas2] M. Kashiwara, Global crystal bases of quantum groups, Duke Math. J. 69 (1993), 455-485.

[KKO] S.-J. Kang, M. Kashiwara and S.-J. Oh, Supercategorification of quantum Kac-Moody algebras II, arXiv:1303.1916.

[KKT] S.-J. Kang, M. Kashiwara and S. Tsuchioka, Quiver Hecke superalgebras, arXiv:1107.1039.

[Kh] M. Khovanov, How to categorify one-half of quantum $\mathfrak{g l}(1 \mid 2)$, arxiv:1007.3517.

[KL] M. Khovanov and A. Lauda, A diagrammatic approach to categorification of quantum groups I, Represent. Theory 13 (2009), 309-347; II, Trans. AMS. 363 (2010), 2685-2700; III, Quantum Topology, 1 (2010), 1-92.

[Kw] J.-H. Kwon, Crystal Bases of q-deformed Kac Modules Over the Quantum Superalgebra $U_{q}(\mathfrak{g l}(m \mid n))$, Int. Math. Res. Notices 2014 (2014), 512-550.

[HP] N. Hu and Y. Pei, Notes on Two-Parameter Quantum Groups, (II), Comm. Alg. 40 (2012), 3202-3220.

[HW] D. Hill and W. Wang, Categorification of quantum Kac-Moody superalgebras, Trans. Amer. Math. Soc. (to appear), arXiv:1202.2769v2.

[Lan] E. Lanzmann, The Zhang transformation and $U_{q}(\operatorname{osp}(1,2 l))$-Verma modules annihilators, Alg. and Repr. Theory 5 (2002), 235-258.

[Lau] A. Lauda, A categorification of quantum $\mathfrak{s l}(2)$, Adv. Math. 225 (2010), 33273424.

[LZ] Libin Li and $\mathrm{Pu}$ Zhang, Twisted Hopf Algebras, Ringel-Hall Algebras, and Green's Categories, Journal of Algebra 231 (2000), 713-743.

[Lu1] G. Lusztig, Canonical bases arising from quantized enveloping algebras, J. Amer. Math. Soc. 3 (1990), 447-498. 
[Lu2] G. Lusztig, Finite dimensional Hopf algebras arising from quantum groups, J. Amer. Math. Soc. 3 (1990), 257-296.

[Lu3] G. Lusztig, Canonical bases in tensor products, Proc. Nat. Acad. Sci. U.S.A. 89 (1992), no. 17, 8177-8179.

[Lu4] G. Lusztig, Introduction to Quantum Groups, Progress in Math. 110, Birkhäuser 1993.

[Ge] N. Geer, Etingof-Kazhdan quantization of Lie superbialgebras, Adv. in Math. 207 (2006), 1-38.

[Gr] J. A. Green, Quantum groups, Hall algebras and quantized shuffles, in "Finite Reductive Groups; Related Structures and Representations" (M. Cabanes, Ed.), Prog. in Math. 141, Birkhäuser (1997), 273-290.

[MW] V. Mikhaylov and E. Witten, Branes and supergroups, to appear.

[MZ] I.M. Musson and Y.-M. Zou, Crystal basis for $U_{q}(\operatorname{osp}(1,2 r))$, J. of Alg. 210 (1998), 514-534.

[ORS] P. Ozsváth, J. Rasmussen, and Z. Szabó, Odd Khovanov homology. arXiv:math.QA/0710.4300

[RT] N. Reshetikhin and V. Turaev, Invariants of 3-manifolds via link polynomials and quantum groups, Invent. Math. 103 (1991), 547-597.

[R] R. Rouquier, 2-Kac-Moody algebras, arxiv:0812.5023.

[VV] M. Varagnolo and E. Vasserot, Canonical bases and KLR algebras, J. Reine Angew. Math. 659 (2011), 67-100.

[Wa] W. Wang, Double affine Hecke algebras for the spin symmetric group, Math. Res. Lett. 16 (2009), 1071-1085. 
[We] B. Webster, Knot invariants and higher representation theory I, arxiv:1309.3796.

[Y1] H.'Yamane, Quantized enveloping algebras associated with simple Lie superalgebras and their universal R-matrices, Publ. Res. Inst. Math. Sci. 30 (1994), 15-87.

[Y2] H. Yamane, On defining relations of affine Lie superalgebras and affine quantized universal enveloping superalgebras, Pub. Res. Inst. Math. Sci. 35 (1999), 321390.

[Z] Y.-M. Zou, Integrable representations of $U_{q}(\operatorname{osp}(1,2 n))$, J. of Pure and Applied Alg. 130 (1998), 99-112. 